\title{
Planetary space weather: scientific aspects and future perspectives
}

\author{
Christina Plainaki ${ }^{1,2, *}$, Jean Lilensten ${ }^{3}$, Aikaterini Radioti ${ }^{4}$, Maria Andriopoulou ${ }^{5}$, Anna Milillo ${ }^{1}$, \\ Tom A. Nordheim ${ }^{6}$, Iannis Dandouras ${ }^{7}$, Athena Coustenis ${ }^{8}$, Davide Grassi ${ }^{1}$, Valeria Mangano ${ }^{1}$, \\ Stefano Massetti ${ }^{1}$, Stefano Orsini ${ }^{1}$, and Alice Lucchetti ${ }^{9}$ \\ 1 INAF-IAPS, via del Fosso del Cavaliere 100, 00133 Rome, Italy \\ 2 Nuclear and Particle Physics Department, Faculty of Physics, National and Kapodistrian University of Athens, \\ 15784 Athens, Greece \\ *Corresponding author: christina.plainaki@iaps.inaf.it \\ 3 Institut de Planétologie et d'Astrophysique de Grenoble, CNRS/UGA, 38041 Grenoble, France \\ 4 Laboratoire de Physique Atmosphérique et Planétaire, Institut d'Astrophysique et de Géophysique, Université de Liège, \\ 4000 Liege, Belgium \\ 5 Space Research Institute, Austrian Academy of Sciences, 8042 Graz, Austria \\ 6 Jet Propulsion Laboratory, California Institute of Technology, 4800 Oak Grove Dr, Pasadena, 91109 CA, USA \\ 7 IRAP, University of Toulouse/CNRS, 31028 Toulouse, France \\ 8 LESIA, Observ. Paris-Meudon, CNRS, Univ. P. et M. Curie, Univ. Paris-Diderot, 92195 Meudon, France \\ 9 CISAS, University of Padova, via Venezia 15, 35131 Padova, Italy
}

Received 27 November 2015 / Accepted 6 June 2016

\begin{abstract}
In this paper, we review the scientific aspects of planetary space weather at different regions of our Solar System, performing a comparative planetology analysis that includes a direct reference to the circum-terrestrial case. Through an interdisciplinary analysis of existing results based both on observational data and theoretical models, we review the nature of the interactions between the environment of a Solar System body other than the Earth and the impinging plasma/radiation, and we offer some considerations related to the planning of future space observations. We highlight the importance of such comparative studies for data interpretations in the context of future space missions (e.g. ESA JUICE; ESA/JAXA BEPI COLOMBO). Moreover, we discuss how the study of planetary space weather can provide feedback for better understanding the traditional circumterrestrial space weather. Finally, a strategy for future global investigations related to this thematic is proposed.
\end{abstract}

Key words. Space weather, Planetary atmospheres, Planetary magnetospheres, Exospheres, Interactions, Comparative planetology, Future missions, JUICE, BEPI COLOMBO

\section{Introduction}

\subsection{Definition of planetary space weather and analogy} with the circum-terrestrial case

In a seminal paper on our understanding of space environments around Solar System bodies other than Earth, Lilensten et al. (2014) discussed the characteristics of planetary space weather, an emerging aspect of the space weather discipline. The earliest definition of space weather, provided by the US National Space Weather Plan $(1995,2000)$, referred to the "conditions on the Sun and in the solar wind, magnetosphere, ionosphere, and thermosphere that can influence the performance and reliability of space-borne and ground-based technological systems and can endanger human life or health". In 2010, this definition was slightly modified and the significant role of the variability of the Sun and of the environment conditions was evidenced: "the term 'space weather' refers to the variable conditions on the Sun, throughout space, and in the Earth's magnetic field and upper atmosphere that can influence the performance of space-borne and ground-based technological systems and endanger human life or health". In both of these definitions, however, the term "space weather" was restricted to the terrestrial case and did not include planetary environments.
To account for this necessity, the US National Space Weather Program (http://www.nswp.gov/, version 25/06/2014) proposed the following definition: "space weather refers to conditions on the Sun and in the space environment that can influence the performance and reliability of space-borne and ground-based technological systems, and can endanger human life or health". Although this new definition referred clearly to both the conditions on the Sun and in the actual space environment of a body, the use of the term "ground-based technological systems" excluded gaseous planets.

In a 2008 definition agreed among 24 countries (Lilensten \& Belehaki 2009), it was stated that "Space Weather is the physical and phenomenological state of natural space environments; the associated discipline aims, through observation, monitoring, analysis and modelling, at understanding and predicting the state of the Sun, the interplanetary and planetary environments, and the solar and non-solar driven perturbations that affect them; and also at forecasting and now-casting the possible impacts on biological and technological systems". We note that in this definition the phenomenon and the associated discipline are clearly distinguished. Moreover, the 2008 definition focuses on space environments stating that planetary space weather cannot be disconnected from the temporal 
variability of either the solar activity or the magnetospheric plasma behaviour at planetary systems. Therefore, the discipline of planetary space weather refers to the study of the variability of planetary (or satellite) environments (e.g. atmospheres, exospheres" (often referred to as "tenuous atmospheres" Johnson et al. 2009), intrinsic magnetospheres) determined by the variability of the solar activity or/and the interplanetary space dynamics (or/and the dynamics of the magnetosphere in which the Solar System body may be embedded).

Recently, the World Meteorological Organisation (WMO) proposed the following definition": "Space Weather is defined as the physical and phenomenological state of the natural space environment including the Sun, the solar wind, the magnetosphere, the ionosphere and the thermosphere, and its interaction with the Earth. The associated discipline aims, through observation, monitoring, analysis and modelling, at understanding the driving processes, predicting the state of the Sun, the interplanetary and planetary environments including the Earth's magnetic field, their disturbances, and forecasting and nowcasting the potential impacts of these disturbances on ground-based or space-based infrastructure and human life or health". This definition re-introduces the Earth as a unique object of study and is therefore not suited for planetary space weather. Nevertheless, a WMO task group has defined the space environment as "the physical and phenomenological state of the natural space environment, including the Sun and the interplanetary and planetary environments", and as meteorology of space "the discipline which aims at observing, understanding and predicting the state of the Sun, of the interplanetary and planetary environments, their disturbances, and the potential impacts of these disturbances on biological and technological systems". ${ }^{3}$ This definition certainly includes the planetary space weather case, nevertheless, it is focused on the Sun as the main driver for the related phenomena. However, at large distances from the Sun, other agents (e.g. cosmic rays, accelerated magnetospheric ions) may be the primary drivers of space weather. In the context of a more global perspective, therefore, it is important to mention both the solar and non-solar driven perturbations in the definition of space weather, especially when referring to planetary space weather. Based on this principle, in the current draft, we consider the 2008 definition of planetary space weather, consistently with the previous work by Lilensten et al. (2014).

Space weather at a planet or satellite is strongly determined by the interactions between the body in question and its local space environment. Different aspects of the conditions at the Sun, and of the solar wind and magnetospheric plasmas at different distances from the Sun, can influence the performance and reliability of space-borne technological systems throughout the Solar System. The study of planetary space weather considers different cross-disciplinary topics, including the interaction of solar wind and/or of magnetospheric plasmas with planetary/satellite surfaces, thick (e.g. at Jupiter, Saturn, Uranus, Mars, Venus, Titan) or tenuous (e.g. at Ganymede, Europa, Mercury, our Moon) atmospheres

\footnotetext{
1 The exosphere is defined as the region of the atmosphere where the particles travel in ballistic trajectories and collisions are improbable (Johnson 1994).

2 Four-year plan for WMO coordination of space weather activities, Towards a Space Weather Watch, Annex to draft resolution 4.2.4(2)/1 (Cg-17, 2015).

${ }^{3}$ WMO report CBS-MG-16-D31, 2016.
}

and ionospheres; the variability of the planetary magnetospheric regions under different external plasma conditions; the interactions of a planet's radiation belts with atmospheres, satellites and rings; space weathering and planetary or lunar surface charging at bodies possessing tenuous atmospheres.

The main agents determining the space weather conditions around a body in the Solar System are:

- the distance of the body from the Sun, determining the properties of solar wind and solar energetic particles (SEPs), the solar photon flux and the properties of the Interplanetary Magnetic Field (IMF) at that location;

- the presence (or absence) of a dense atmosphere;

- the optical thickness (wavelength dependent) of the body's atmosphere and the mean free paths for neutrals and ions travelling therein;

- in case of the Giant planet moons, the existence of a strong (or weak) magnetosphere in which the body is embedded (e.g. Europa and Ganymede; Titan and Enceladus versus Miranda and Ariel; Naiade, Talassa and Triton);

- the possession by the body of an intrinsic or induced magnetic field;

- the existence of endogenic sources (e.g. plumes, volcanoes);

- galactic cosmic rays (GCRs);

- micrometeorites and dust.

Any variability related to the energy release from the Sun, in the form of photon flux (in the UV, EUV and X-ray bands), solar wind (streams), coronal mass ejections (CMEs) and SEPs, has been known to be the principal agent determining circum-terrestrial space weather. At other planetary bodies in the Solar System, solar-driven perturbations also play a role in atmospheric and magnetospheric processes, however the details of such interactions are planet-dependent and in some cases (e.g. Jupiter), internal processes dominate over external drivers. In particular, in the outer Solar System, the variability of internal (to the planetary system) plasma sources can be the main driver for space weather in these environments. For example, in the Jovian system, the variability of the plasma originating from Io dynamically affects the charged particle environment at the Galilean moons (Bolton et al. 2015). Moreover, interstellar pick-up ions (IPUIs) can change the dynamics and composition of the solar wind at different locations in the Solar System, and may therefore, also to some extent, affect planetary space weather (Lilensten et al. 2014). In general, a wide range of timescales characterize the variability of perturbations, both solar and non-solar in origin. This results in space weather phenomena of different intensities and morphologies across the entire Solar System.

\subsection{Motivation for research}

Radiation effects on spacecraft instruments, both in terms of electromagnetic waves and particles, have been often discussed in the past (see review by Landis et al. 2006; Boudjemai et al. 2015) and represent the major technological impact of planetary space weather. The available knowledge of the characteristics of radiation environments at different planetary systems is based on in-situ and remote sensing measurements from flybys as well as orbiting spacecraft. The largest datasets 
exist for Earth, followed by Saturn, Jupiter and Mercury (Krupp 2015), whereas important information has also been obtained from ground-based or Earth-orbiting telescopes as well as analytical and numerical models. The variability of the spacecraft radiation environment due to major space weather events has often resulted in either a partial or a complete failure of the detection systems on board. One such example is the radiation detector of the Mars Radiation Environment Experiment (MARIE) on-board Mars Odyssey (Andersen 2006), which was presumed to have failed due to damage from the unusually intense SEP events of OctoberNovember 2003. Another example involved the Nozomi spacecraft, which suffered disruptions to its communication system due to a solar flare occurring in April 2002 (Miyasaka et al. 2003). Another major space weather event resulted in an increase in background radiation that made it difficult for the Analyser of Space Plasmas and Energetic Atoms 3 (ASPERA-3) instrument on-board Mars Express (MEX) to evaluate ion escape fluxes at Mars (Futaana et al. 2008). Due to these phenomena, there is an increasing need for an overall effort to understand, monitor and potentially predict (to the extent that the latter is possible) the actual conditions around the planetary environment under investigation. In order to achieve the maximum benefit from such an effort, it is necessary to extend the methodological frameworks of circum-terrestrial space weather to different contexts. Therefore, the strongest motivation for the current scientific review is the need for a synoptic organization of the available knowledge on the field of interactions at different planetary systems, in parallel with a comparative analysis encompassing the inter-connection among planetary space weather aspects belonging to different disciplines (e.g. plasma variability and its effects on atmospheric heating).

Among others, the future ESA JUpiter ICy moons Explorer (JUICE) and the ESA/JAXA BEPI COLOMBO (composed of two spacecraft in orbit around Mercury) space missions (to be launched in 2022 and 2018, respectively) will provide the opportunity to study space weather phenomena at two different regions of our Solar System, dominated by essentially different conditions. Moreover, among the principal science objectives of JUICE are the investigation of the near-moon plasma and neutral environments as well as the characterization of Jupiter's atmosphere and magnetosphere (Grasset et al. 2013). In this view, a synopsis of the plasma variability in the giant planet's magnetosphere and its effects on the exospheres of the moons and the atmosphere of the planet itself is a useful tool for any related study; moreover, such an effort is rather urgent since it could be of help during the definition of the mission's observational strategies as well as during the implementation of related models. Furthermore, the results acquired by the Cassini mission have demonstrated that the plasma variability in the Saturn system is an important agent in the interactions within the planet's magnetosphere and atmosphere as well as in the moon and ring environments (André et al. 2008; Jia et al. 2010). The interactions between the Enceladus (Fleshman et al. 2010) and possibly Europa (Roth et al. 2014a) plumes with the magnetospheric environments are two other important examples of planetary space weather phenomena about which our knowledge is currently limited. In addition, the Uranus case is perhaps one of the most intriguing environments in the outer Solar System for planetary space weather studies, mainly due to the particular orientation of the solar wind direction, relative to the planet's rotation axis and magnetic field. The determination of the space weather conditions in the Solar System is particularly challenging and requires as a first step the definition of key observable quantities. With this in mind, it is clear that there is a strong need for detailed space weather studies of individual planetary environments. In summary, the planetary space weather discipline aims at:

- understanding the nature of planetary environments revealing the physics of their interactions with the solar wind and/or the magnetospheric environments to which they are embedded;

- forecasting and nowcasting the space environment variability around a body in the Solar System;

- predicting the impacts of space environments on technological systems on-board spacecraft and/or eventually on humans.

The scope of this review paper is to integrate the effort started in the earlier study by Lilensten et al. (2014) by (a) providing an update of the current knowledge on the scientific aspects of planetary space weather, with special emphasis on the outer Solar System; (b) discussing considerations related to future missions such as ESA JUICE and ESA/ JAXA BEPI COLOMBO and (c) demonstrating the benefit for the circum-terrestrial space weather discipline from studies related to other planetary environments. In Section 2, we present a brief review of the interactions taking place at different Solar System bodies, demonstrating that a "general approach" to the topic of planetary space weather cannot be easily adopted; in Sections 2.1 and 2.2 we review the outer and inner Solar System cases, respectively. In Section 3, we discuss some aspects of space weather from a comparative planetology viewpoint and give some considerations related to the planning of future observations both with Earth-orbiting telescopes and instruments on-board spacecraft at different planetary environments. The importance of such investigations for the interpretation of in-situ observations is discussed extensively. Although in the current paper we focus on topics related to the upcoming missions ESA JUICE and ESA/JAXA BEPI COLOMBO, we emphasize the relevance of such planetary space weather studies for future missions to all destinations in the Solar System. In Section 4, we discuss the benefit from planetary space weather studies for the circum-terrestrial space weather research. The conclusions of this study are presented in Section 5. We underline that in the current paper, interactions between comets and their external environment, as well as interactions at the PlutoCharon system have been intentionally omitted due to continuous updates in these fields, provided by the ESA Rosetta and NASA New Horizons missions, respectively.

\section{Space weather interactions in the Solar System}

The interactions of different Solar System bodies with solar wind and/or magnetospheric plasma have different spatial scales and timescales. Moons can be submerged in their parent body's magnetosphere either permanently (e.g. Ganymede) or for a fraction of their orbit (e.g. Titan). In the Jupiter and the Saturn systems, the speed of the corotating (with the planet) plasma is higher than the orbital velocity of some satellites. As a result, the thermal corotating plasma overtakes these moons. For example, the orbital speed of Enceladus is $12.6 \mathrm{~km} / \mathrm{s}$ while the thermal plasma corotates with Saturn at $39 \mathrm{~km} / \mathrm{s}$ near Enceladus and thus the corotating plasma 
(a)

(b)

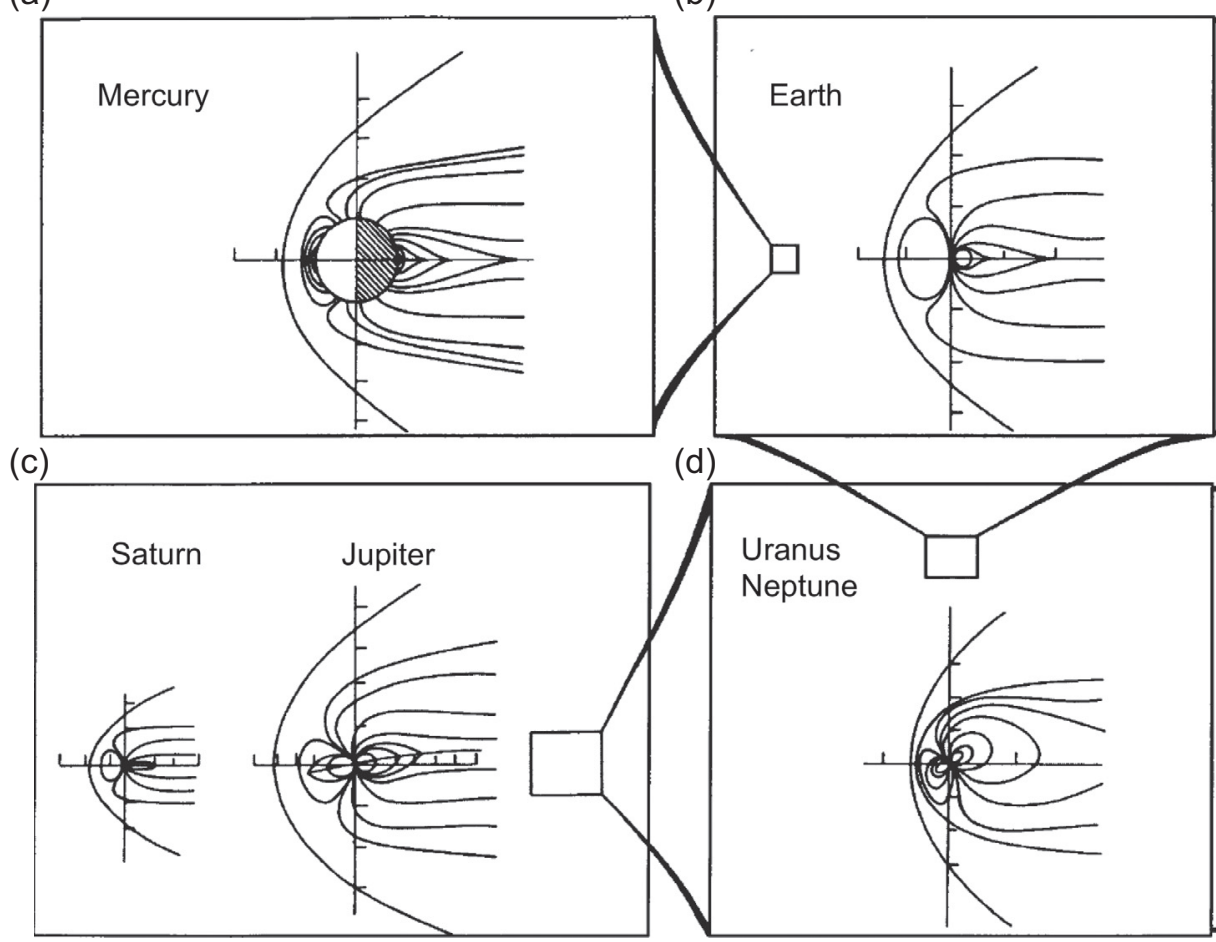

Fig. 1. Comparison of the sizes of magnetospheres of the outer planets, the Earth and Mercury. Boxes illustrate the relative size of the magnetospheres. The conical at the left of each planet represents the bow shock, whereas the swept-back lines represent the planetary magnetic field. (a) Mercury. (b) The Earth. (c) Saturn (left) and Jupiter (right). (d) Uranus or Neptune. Adapted from Kivelson \& Russell (1995).

overtakes this moon forming a "corotational wake" at the hemisphere leading the moon's orbital motion. The Cassini Plasma Spectrometer (CAPS) registered a strong enhancement in the flux of plasma ions when passing upstream of this wake.

In general, the interactions between Solar System bodies and their space environment depend strongly on whether the body is magnetized or unmagnetized. In Figure 1, the magnetospheres of the four outer planets, the Earth and Mercury are presented.

A typical case study for the outer Solar System is Jupiter and its moons Europa, a satellite without any intrinsic magnetic field, and Ganymede, not only the largest moon but also the only known satellite in the Solar System possessing its own magnetosphere. Leading/trailing hemisphere albedo and colour ratio asymmetries have been previously reported for both Ganymede (Clark et al. 1986; Calvin et al. 1995) and more significantly for Europa (McEwen 1986; Sack et al. 1992). For Ganymede, the leading hemisphere has a measurably higher ratio in the green/violet colour ratio $(\sim 0.79)$ than the trailing hemisphere $(\sim 0.73)$, implying a greater abundance of fine-grained frost on the leading hemisphere. Additionally, images of Ganymede acquired by Voyager 2 revealed that Ganymede's high-latitude regions are noticeably brighter than its equatorial regions (Smith et al. 1979). Khurana et al. (2007) showed that there is a clear link between plasma bombardment and polar cap brightening at Ganymede and suggested that sputter-induced redistribution and subsequent cold trapping of water molecules is responsible for the observed bright and dark patches of the bright polar terrain. These authors also suggested that leading vs. trailing brightness differences in Ganymede's low-latitude surface are due to enhanced plasma flux onto the leading hemisphere, rather than to darkening of the trailing hemisphere. This is because the equatorial region is shielded from electrons over all longitudes, whereas in the leading sector, magnetic reconnection (i.e. the breaking and topological rearrangement of magnetic field lines in a plasma, resulting in the conversion of magnetic field energy to plasma kinetic and thermal energy) could lead to some sputtering of surface material at low latitudes from recently trapped particles; in those regions, however, such a process is expected to have lower rates than in the polar caps. Recent simulations by Plainaki et al. (2015) of Ganymede's sputter-induced water exosphere support this scenario. In contrast to Ganymede, the entire surface of Europa is bombarded by Jovian plasma (maximum precipitating flux is at the trailing hemisphere apex), suggesting that sputter-induced redistribution of water molecules is a viable means of brightening the satellite's surface. As a consequence of these space weather conditions at Europa, the spatial distribution of the generated $\mathrm{H}_{2} \mathrm{O}$ exosphere is expected to follow the pattern of the ion precipitation to the surface, being most intense above the moon's trailing hemisphere (see Plainaki et al. 2012, 2013). On the basis of the above paradigm, it becomes clear that a "general approach" to the topic of planetary space weather, determined by the interactions between the body's environment and its surrounding space, is not straightforward.

\subsection{Space weather in the outer Solar System}

All planets in the outer Solar System possess magnetic fields, which can be assumed to have approximately either a dipole form (e.g. Jupiter and Saturn, with tilt angles $9.6^{\circ}$ and $0^{\circ}$, respectively) or a multipole form (e.g. Uranus and Neptune, with tilt angles $59^{\circ}$ and $47^{\circ}$, respectively). Space weather at the environments of the outer planets depends on one hand 
on the solar wind density and the IMF intensity and direction, and on the other hand on the planetary magnetic field tilt and plasma pressure inside the magnetosphere. Below we will discuss the different characteristics of these magnetospheres related to space weather.

\subsubsection{Space weather at Uranus, Neptune and exoplanetary analogues}

Neptune and Uranus have strong non-axial multipolar magnetic field components compared with the axial dipole component (Connerney et al. 1991; Herbert 2009). These highly nonsymmetric internal magnetic fields (Ness et al. 1986, 1989; Connerney et al. 1991; Guervilly et al. 2012), coupled with the relatively fast planetary rotation and the unusual inclination of the rotation axes from the orbital planes, imply that the magnetospheres of Uranus and Neptune are subject to drastic geometrical variations on both diurnal and seasonal timescales. The relative orientations of the planetary spin, the magnetic dipole axes and the direction of the solar wind flow determine the configuration of each magnetosphere and, consequently, the plasma dynamics. Space weather phenomena, therefore, at these environments are expected to be significantly variable in intensity and morphology over a range of timescales. The study of the interactions responsible for space weather phenomena at the environments of the icy giants can also be used as a template for exoplanetary investigations.

On the basis of the Voyager 2 occultation measurements, the atmosphere of Uranus up to the vicinity of the exobase (i.e. near $\sim 1.25 R_{\mathrm{U}}$, where $R_{\mathrm{U}}$ is the planet's radius) consists mainly of $\mathrm{H}_{2}$ (Herbert et al. 1987). At higher altitudes, atomic $\mathrm{H}$ becomes the major constituent. The Voyager IR spectrometer deduced also a He content of about $15 \%$ (see Fig. 2). At the current moment, our knowledge on space weather phenomena at Uranus is essentially based on (a) a limited set of plasma, FUV and IR measurements obtained during the Voyager 2 flyby (Herbert 2009), (b) a series of observations of the FUV and IR aurora with the Hubble Space Telescope (HST; Ballester 1998) and (c) observations from ground-based telescopes (e.g. Trafton et al. 1999). Voyager 2 provided a single snapshot of the Uranian magnetosphere at solstice, when the angle of attack between the solar wind axis and the magnetic dipole axis varied between $68^{\circ}$ and $52^{\circ}$, to some extent similar to the situation at the Earth's magnetosphere (Arridge et al. 2014). Moreover, the Voyager 2 plasma observations showed that when the Uranus dipole field is oppositely directed to the interplanetary field, magnetic plasma injection events are present in the inner magnetosphere, likely driven by reconnection every planetary rotation period (Sittler et al. 1987). However, the near alignment of the rotation axis with the planet-Sun line during solstice means that plasma motions produced by the rotation of the planet and by the solar wind are effectively decoupled (Selesnick \& Richardson 1986; Vasyliunas 1986) hence the related space weather phenomena are expected to differ substantially from those at Earth, both in timescales and spatial morphology. Due to the planet's large obliquity, near equinox, Uranus' asymmetric magnetosphere changes between nearpole-on and near-perpendicular configurations, every quarter of a planetary rotation. The configuration of Uranus' magnetosphere in the 2030s is shown in Figure 3.

The available in-situ measurements of space weatherrelated interactions in the Uranian system refer to a single configuration between the planet and the solar wind flow. Therefore, the time-dependent modulation of the magnetic (a)

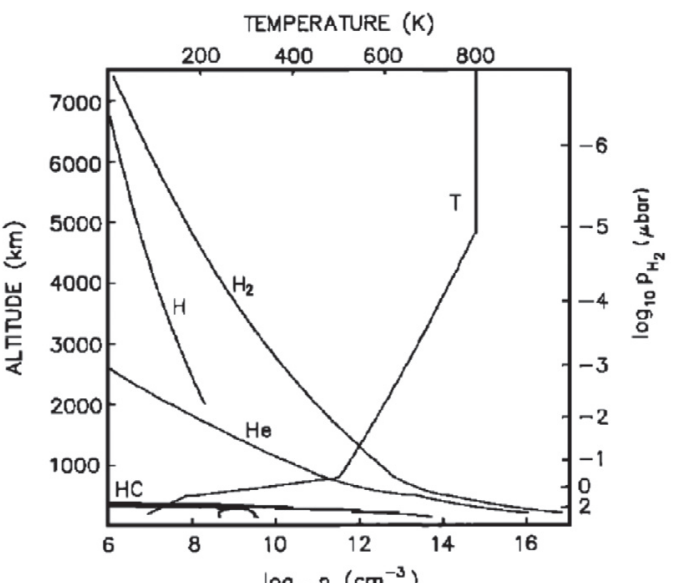

(b)

$\log _{10} n\left(\mathrm{~cm}^{-3}\right)$

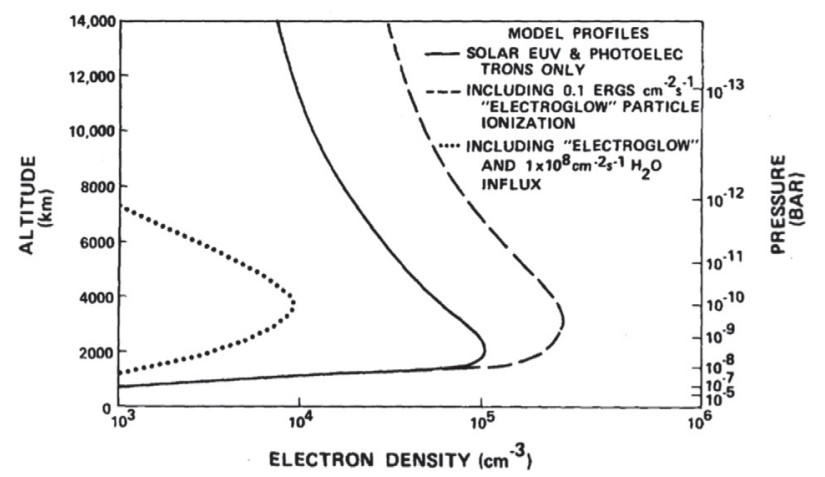

Fig. 2. (a) Abundance and temperature profiles of the main constituents of Uranus upper atmosphere, as inferred from the occultation observations by the Voyager 2 ultraviolet spectrometer. Helium was not detectable in the occultations hence it has been extrapolated upwards from IRIS measurements (Hanel et al. 1986). From Herbert et al. (1987). (b) Modelled ionospheric profiles of electron densities for Uranus using the Voyager inferred temperature profiles. From Waite \& Cravens (1987)

reconnection sites, the details of the solar wind plasma entry in the inner magnetosphere of Uranus, the plasma precipitation to the exosphere and ionosphere, the modes of interaction (pickup, sputtering and charge exchange) between the magnetospheric plasma and the rings and moons of Uranus are largely unknown. Proton and electron radiation belts (with energies up to tens of $\mathrm{MeVs}$ ), albeit slightly less intense than those at Saturn, were also observed in the inner magnetosphere of Uranus (Cheng et al. 1991; Mauk \& Fox 2010) but their diurnal and seasonal variability is also unknown to a great extent (Arridge et al. 2014). Recent observations and theoretical works on this thematic suggest a limited role for the solar wind-driven dynamics at equinox (Lamy et al. 2012; Cowley 2013) and solar wind-magnetosphere coupling via magnetic reconnection that varies strongly with season and solar cycles (Masters 2014). At the moment, therefore, it is not clear if space weather phenomena are determined predominantly by solar wind or by a more rotation-dominated magnetosphere, such as the one at Jupiter or Saturn (Arridge et al. 2014). Future missions to Uranus with the science goal, among others, to understand the solar wind-magnetosphere-ionosphere coupling and its role in determining the space weather conditions at the icy giant, have been recently proposed (Arridge et al. 2012, 2014; Turrini et al. 2014).

The aurora of Uranus is perhaps the most significant evidence of how space weather conditions around the planet 
(a)

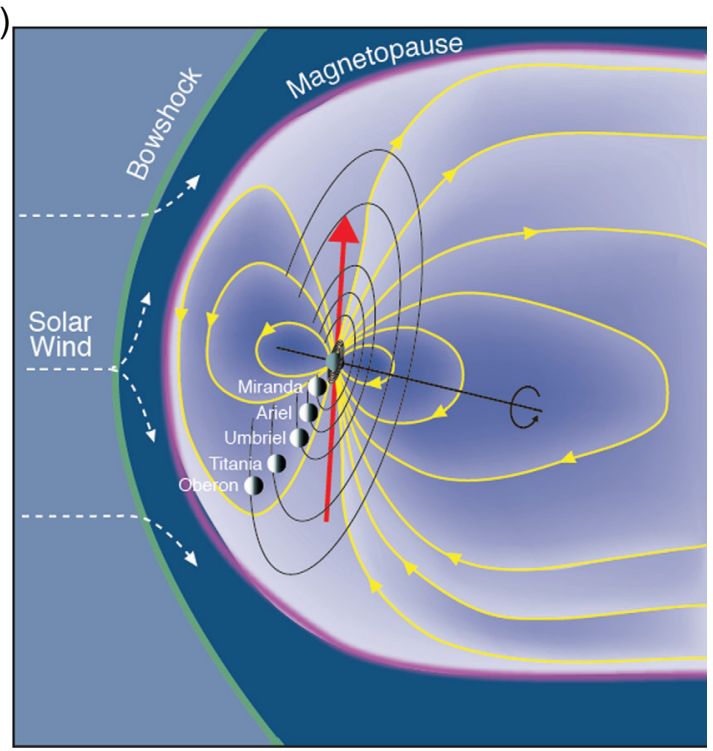

(b)

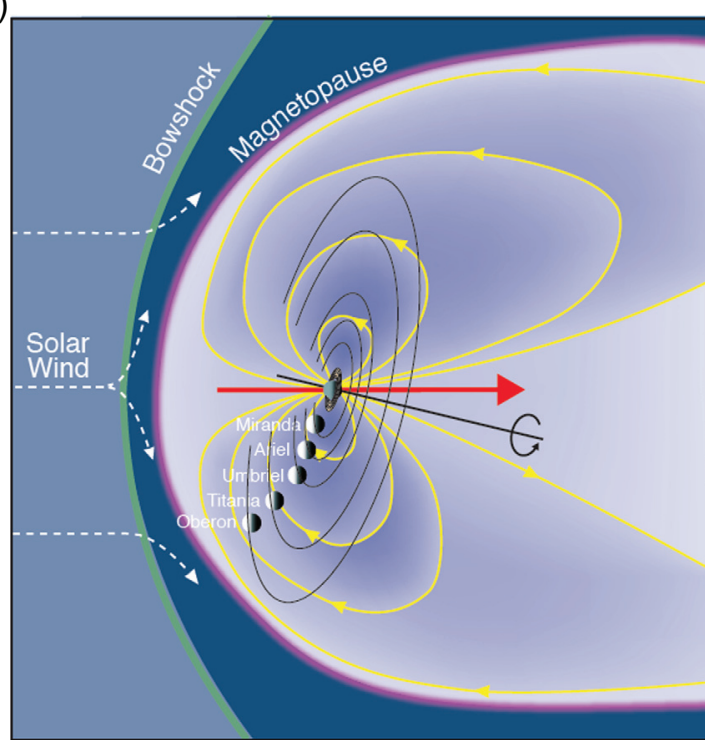

Fig. 3. The magnetosphere of Uranus in the late 2030s between solstice and equinox where the rotation axis has a significant out-ofplane component. Planetary rotation results in significantly different magnetosphere configurations. The angle between the magnetic dipole vector (red arrow) and the planet's rotational plane is $\sim 59^{\circ}$. Credit: Chris Arridge, Fran Bagenal and Steve Bartlett.

vary. It is underlined that one of the major open questions regarding Uranus is the determination of the exact mechanism providing the required additional heating of the planet's upper atmosphere. In a recent study, Barthelemy et al. (2014) analysed HST FUV spectral images obtained in November 2011 (Lamy et al. 2012) and in 2012, with the scope to extract any possible $\mathrm{H}_{2}$ emission produced in the upper atmosphere of the planet. For their interpretations of the data, they used simulated $\mathrm{H}_{2}$ emissions created by energetic particle precipitation in the upper atmosphere. They found that for the spectrum of November 2011, when an auroral spot was positively detected, a small contribution of $\mathrm{H}_{2}$ emission was identified providing, hence, evidence for the presence of precipitating electrons, giving a total energy input of at least $150 \mathrm{GW}$. In the context of space weather, it is important to note that Barthelemy et al. (2014) did not find any evidence for auroral electron-induced emission above the detection threshold in any other spectra except for those corresponding to November 2011. Future in-situ plasma and energetic neutral particle observations could provide significant feedback for the determination of the planet's space weather conditions and for determining if they indeed have a key role in this context. The planetary period modulation of the field-aligned currents at Uranus may lead to modulated auroral electron acceleration in regions of upward currents, as it happens in the case of Saturn (e.g., Nichols et al. 2008, 2010a, 2010b; Southwood \& Kivelson 2009; Badman et al. 2012; Hunt et al. 2014); associated modulated radio emissions at kilometre wavelengths generated via the cyclotron maser instability could be present (Zarka 1998; Lamy et al. 2010, 2011). Such observations can be essential for the identification of space weather phenomena at Uranus.

An additional particle source, at least in the inner magnetosphere of Uranus, may be the Cosmic Ray Albedo Neutron Decay (CRAND), responsible for the $\mathrm{MeV}$ ion spectra changes as a function of dipole $\mathrm{L}$, where $\mathrm{L}$ is the McIlwain parameter $^{4}$ (Krupp 2015). The CRAND source has also been suggested and confirmed to be a source for both Jupiter's (Bolton et al. 2015) and Saturn's radiation belts (Kollmann et al. 2013). Given the variability of the magnetosphere configuration at Uranus, the CRAND source may have a significant role in determining the space weather conditions around the planet, especially in the inner magnetosphere regions.

The magnetospheric interaction with the Uranian moons and rings, basically unknown at the current moment, is the agent of space weather at these systems. However, the Voyager data did not provide any evidence for such phenomena. Space weather phenomena occurring due to coupling between the planet's magnetosphere and the expected tenuous atmospheres around the Uranian moons and rings could be identified and studied through in-situ measurements of magnetic field, charged particles and surface-released neutrals. Moreover, remote imaging of charge-exchange energetic neutral atoms (ENAs) would offer a unique opportunity to monitor the plasma circulation where moons and/or Uranus' exosphere are present (Turrini et al. 2014). However, Krupp (2015) noticed that the highly variable inclination of the Uranian moons with respect to the magnetic equator of the planet could lead to highly variable interactions between magnetospheric populations and moon surfaces. In this case, water particles could be sputtered and hence contribute to the magnetospheric population once they get ionized, determining the space weather conditions in the moons' vicinity.

Neptune is a relatively weak source of auroral emissions at UV and radio wavelengths (Broadfoot et al. 1989; Bishop et al. 1995; Zarka et al. 1995, Zarka 1998). Although this nonobservation does not rule out an active magnetosphere, it rules out processes similar to those associated with the aurora observed at Uranus. Whereas the plasma in the magnetosphere of Uranus has a relatively low density and is thought to be primarily of solar wind origin, at Neptune, the distribution of plasma is generally interpreted as indicating that Triton is a major source (Belcher et al. 1989; Krimigis et al. 1989; Mauk et al. 1991, 1995; Richardson et al. 1991). Neptune's satellite Triton, the largest of the 14 known Neptunian moons, possesses a nitrogen and methane atmosphere with a surface pressure of 14 microbar and temperature of about $35.6 \mathrm{~K}$,

\footnotetext{
${ }^{4}$ The L-shell or McIlwain parameter describes the set of magnetic field lines which cross the planet's magnetic equator at the distance of a number of planetary radii equal to the $L$-value.
} 
extending to $950 \mathrm{~km}$ above the surface. The exosphere temperature reaches $95 \mathrm{~K}$ (Coustenis et al. 2010). Most of the atmosphere contains a diffuse haze, which probably consists of hydrocarbons and nitriles produced by the photolysis of $\mathrm{N}_{2}$ and $\mathrm{CH}_{4}$ (McKinnon \& Kirk 2007). Therefore, space weather at Neptune could be substantially the result of the interaction between Triton-originating plasma and Neptune's magnetosphere and atmosphere systems. It is underlined that escape of neutral hydrogen and nitrogen from Triton maintains a large neutral cloud (Triton torus) that is believed to be source of neutral hydrogen and nitrogen (Decker \& Cheng 1994). The escape of neutrals from Triton could be an additional plasma source for Neptune's magnetosphere (through ionization). An agent for possible space weather phenomena at Triton's vicinity is possibly the presence of active geysers, a source of ions for the whole system, similar to the one at Saturn's moon Enceladus (Porco et al. 2014). Since our knowledge of the plasma dynamics in the magnetosphere of Neptune as well as on the neutral particle production in Triton's atmosphere is limited (it is based only on a single flyby by Voyager 2 in 1989), new in-situ plasma and energetic neutral particle observations focused on Triton's region could be of particular importance for future studies related to planetary space weather in the outer Solar System. Space weather phenomena at Neptune's moons depend on combined effects of photoionization, electron impact ionization and charge exchange in the context of a complex coupling between an asymmetric planetary magnetosphere and satellite exospheres at large distances from the Sun.

\subsubsection{Space weather in the Saturnian system}

Saturn is the second largest planet in our Solar System, following Jupiter. Similar to the Jovian case, Saturn is a fast rotating system, with a rotation of approximately $10.5 \mathrm{~h}$ (Gombosi et al. 2009). Above the homopause, $\mathrm{H}_{2}, \mathrm{H}$ and $\mathrm{He}$ dominate the upper atmosphere (see Fig. 4). Space weather at Saturn is manifested by the occurrence of aurorae and thermospheric emissions providing insights into the state of the planet's thermosphere and ionosphere (Fig. 5).

The interaction of Saturn's magnetic field with the solar wind generates a giant magnetosphere which is dynamically and chemically coupled to all other components of Saturn's environment: the rings, the exosphere, the icy satellites (and their tenuous atmospheres), the Enceladus' plumes and Titan's upper atmosphere. It is thus dominated by numerous interactions between charged particles, neutral gas and dust in addition to the usual interactions with the solar wind (Blanc et al. 2002). Saturn's magnetosphere is believed to be an intermediate case between the magnetosphere of the Earth and the one of Jupiter (Andriopoulou et al. 2014). A unique feature is that it is neutral-dominated. The neutral-to-ion ratio is roughly 60 , for radial distances ranging from $3 R_{\mathrm{S}}$ to $5 R_{\mathrm{S}}$ (with $R_{\mathrm{S}}$ being Saturn's radius). This ratio increases for larger and smaller distances (Mauk et al. 2009; Melin et al. 2009; Shemansky et al. 2009). The density profiles of $\mathrm{H}_{2} \mathrm{O}$ and atomic $\mathrm{H}$ peak near the Enceladus orbit at a distance of $\sim 3.95 R_{\mathrm{s}}$ from the planet (Perry et al. 2010). Other Saturnian satellites residing in the planet's magnetosphere can act both as plasma sinks and sources. Observations from early flyby missions to Saturn revealed a very complex ring system with several gaps, where the energetic particle density drops, mainly due to the satellites' presence. The ring system itself (Johnson

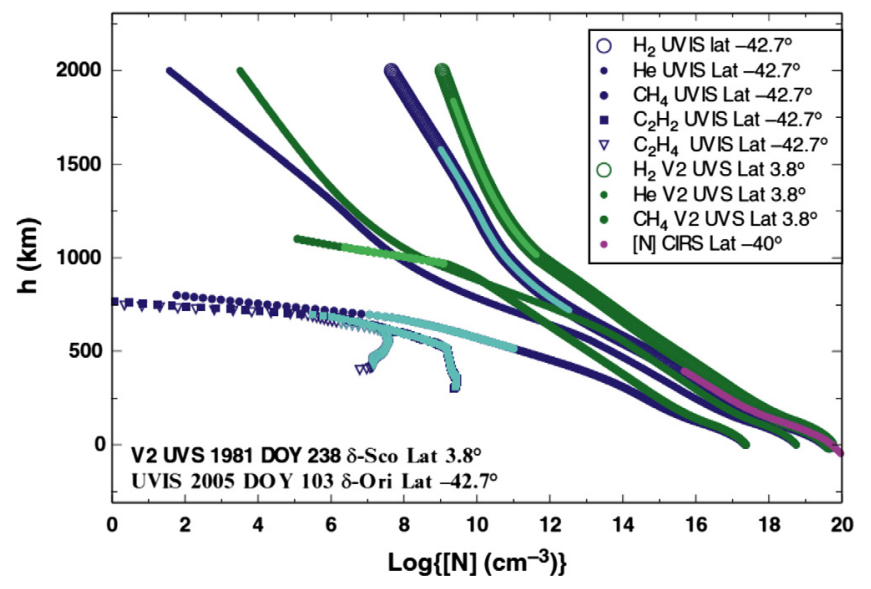

Fig. 4. Density profiles of various components of Saturn's upper atmosphere obtained from forward modelling of the Cassini UVIS $\delta$-Ori stellar occultation on 2005 DOY 103 at a latitude of $-42.7^{\circ}$. From the book Saturn from Cassini-Huygens (M.K. Dougherty et al. eds.), Chapter 8 by Nagy et al. (2009).

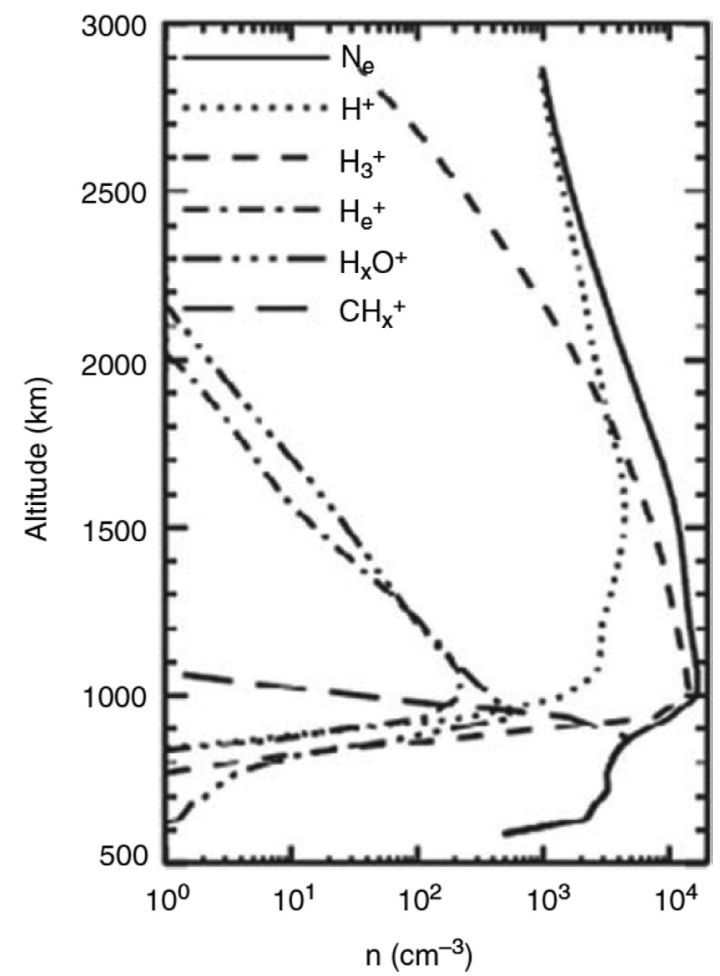

Fig. 5. Noon ion density profiles at Saturn. From the book Saturn from Cassini-Huygens (M.K. Dougherty et al. eds.), Chapter 8 by Nagy et al. (2009).

et al. 2006) and the planet's thermosphere (Shemansky et al. 2009) can be additional sources supplying neutrals in Saturn's magnetosphere. Indeed, a "ring ionosphere" was detected by the CAPS instrument on-board Cassini (Coates et al. 2005).

The investigation of the magnetopause reconnection driven by the solar wind started in the Voyager era. However, its significance is a subject of large debate to date. Voyager observations showed reconnection signatures at Saturn's magnetopause (Huddleston et al. 1997) and suggested that bursty reconnection similar to flux transfer events at Earth (Russell \& Elphic 1979) is not a significant mechanism at 
Saturn, because of the high magnetosonic Mach numbers ${ }^{5}$ which are reached close to Saturn. On the other hand, Cassini plasma and magnetic field observations revealed signatures of reconnection at Saturn's magnetopause (McAndrews et al. 2008). Recently, it was suggested that only a limited fraction of the magnetopause surface could become open (Masters et al. 2012). Moreover, recent studies indicated that reconnection has a less important role at Saturn than at the Earth, in large-scale transport near the subsolar region of the magnetopause (Lai et al. 2012). Finally, several auroral studies provide evidence for the importance of solar wind driven reconnection at Saturn's magnetopause (see among others Badman et al. 2011; Radioti et al. 2011b; Jasinski et al. 2014; Meredith et al. 2014).

\subsubsection{Solar wind effects on the aurora}

The main auroral emission at Saturn (Fig. 6) can be associated with the solar wind-magnetosphere interaction through the generation of field-aligned currents and plasma precipitation (e.g. Cowley et al. 2004), similar to the terrestrial aurora (e.g. Paschmann et al. 2002). Particularly, Cassini measurements in comparison with conjugated auroral observations suggest that the main auroral emission at Saturn is produced by the magnetosphere-solar wind interaction, through the shear in rotational flow across the open-closed field line boundary (OCFB; e.g. Cowley \& Bunce 2001; Bunce et al. 2008). The morphology of Saturn's aurora is demonstrated to respond to solar wind changes (Grodent et al. 2005; Clarke et al. 2009), controlled by the balance between the magnetic field reconnection rate at the dayside magnetopause and the reconnection rate in the nightside tail (Cowley et al. 2004; Badman et al. 2005, 2014).

HST observations (Gérard et al. 2004, 2005) and theoretical studies (Bunce et al. 2005) showed that reconnection occurring at the dayside magnetopause could give rise to bright UV auroral emissions at Saturn observed occasionally near noon, similar to the "lobe cusp spot" at Earth (i.e. emissions located at the cusp magnetic foot point, Fuselier et al. 2002). The magnetospheric "cusp" is the magnetospheric region where magnetosheath plasma has direct access to the ionosphere through reconnection. Specifically, it was proposed by Bunce et al. (2005) that pulsed reconnection at the low-latitude dayside magnetopause for northward-directed IMF is giving rise to pulsed twin-vortical flows in the magnetosphere and ionosphere in the vicinity of the OCFB lines. For the case of southward IMF and high-latitude lobe reconnection, bipolar field-aligned currents are expected, associated with auroral intensifications poleward of the OCFB lines.

Cassini Ultraviolet Imaging Spectrograph (UVIS) auroral observations revealed signatures of consecutive reconnection events at Saturn's magnetopause (Radioti et al. 2011b) in the form of bifurcations of the main emission (Fig. 6a). In the same study it was suggested that magnetopause reconnection could lead to a significant increase of the open flux within a couple of days. In particular, each reconnection event is estimated to open $\sim 10 \%$ of the flux contained within the polar cap. Additionally, Cassini multi-instrument observations, including auroral UV and IR data, confirmed that the auroral

\footnotetext{
5 The magnetosonic Mach number, $M_{f}$, is defined as the upstream (with respect to a collisionless shock) flow speed divided by the speed of the fast magnetosonic wave, which steepens to form the shock (Masters et al. 2011). $M_{f}$ is always $>1$ for shocks, by definition.
}

arcs are related to bursty reconnections at Saturn involving upward field-aligned currents (Badman et al. 2012) and suggested that these are efficient in transporting flux (Badman et al. 2013). A more recent study based on conjugated IMF and HST observations (Meredith et al. 2014) provided evidence of significant IMF dependence on the morphology of Saturn's dayside auroras and the bifurcated arcs. Finally, the auroral evidence of magnetopause reconnection at multiple sites along the same magnetic flux tube similar to the terrestrial case (Fasel et al. 1993) was shown to give rise to successive rebrightenings of auroral structures (Radioti et al. 2013b). The same auroral features, together with magnetospheric measurements, were discussed in Jasinski et al. (2014) in terms of cusp signatures at Saturn's high-latitude magnetosphere.

However, it should be noted that the significance of magnetopause reconnection at Saturn is under debate (Masters et al. 2012). Alternatively to solar wind driven reconnection, the viscous interaction of the solar wind with the planetary magnetosphere, which involves magnetic reconnection on a small scale (Delamere \& Bagenal 2013), influences Saturn's magnetopause dynamics. Cassini UVIS observations revealed recently the presence of small-scale structures in the dayside main auroral emissions indicative of magnetopause KelvinHelmholtz instabilities, which are key elements of the solar wind-magnetosphere viscous interaction (Grodent et al. 2011).

In addition to dayside magnetopause, the solar wind influences Saturn's magnetotail and this interaction leaves its footprint in the aurora. It has been suggested that Saturn's magnetotail is influenced by a combination of solar wind (Dungey 1961, like at Earth) and internally driven magnetic reconnection (Vasyliunas 1983) as well as viscous interaction of the solar wind with the planetary magnetosphere (Delamere \& Bagenal 2013). Tail reconnection in the Dungey-cycle manner is expected to result in bright and fast rotating aurorae, which expand poleward in the dawn sector, reducing significantly the size of the polar cap and thus resulting in the closure of flux (Badman et al. 2005; Cowley et al. 2005; Jia et al. 2012).

Particularly, intense auroral activity in the dawn auroral sector was recently observed by Cassini/UVIS and was characterized by significant flux closure with a rate ranging from 200 to $1000 \mathrm{kV}$ (Radioti et al. 2014). Additionally, Nichols et al. (2014) based on HST observations revealed auroral intensifications in the dawn auroral sector, propagating at 3.3 times the rigid corotation. These intensifications were suggested to be indicative of ongoing, bursty reconnection of lobe flux in the magnetotail, with flux closure rates of $280 \mathrm{kV}$ (Fig. 6b). Badman et al. (2015) reported an event of solar wind compression that impacted Saturn's magnetosphere, identified by an intensification, and extension to lower frequencies, of the Saturn kilometric radiation. This event was manifested in the auroral dawn region as a localized intense bulge of emission followed by contraction of the nightside aurora. These observations are interpreted as the response to tail reconnection events, initially involving Vasyliunas-type reconnection of closed mass-loaded magnetotail field lines, and then proceeding onto open lobe field lines, causing the contraction of the polar cap region on the nightside. Additionally, Radioti et al. (2016) revealed multiple intensifications within an enhanced auroral dawn region suggesting an $x$-line in the tail, which extends from 2 to 5 LT. Such UV intensifications have been also previously suggested to be associated with depolarizations in the tail (Jackman et al. 2013). The localized enhancements reported by Radioti et al. (2016) evolved 
(a)

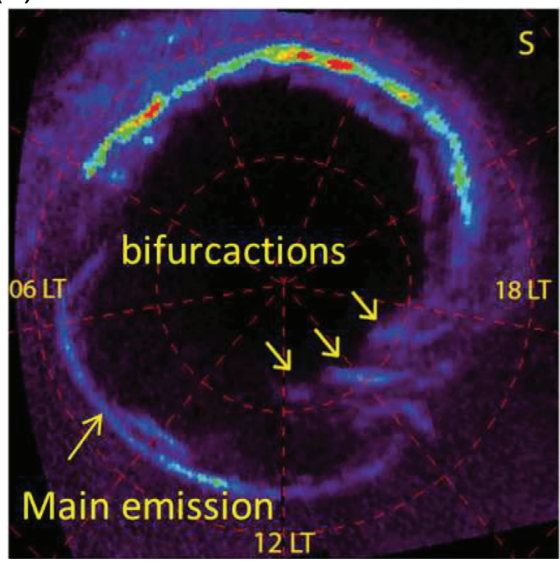

(b)

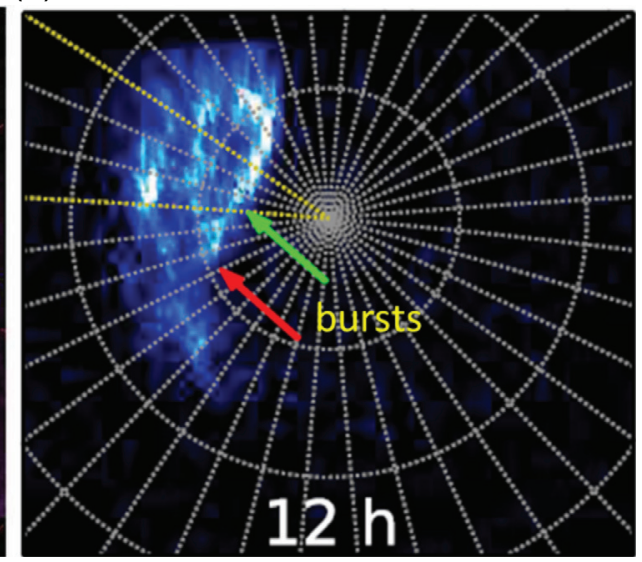

Fig. 6. (a) Polar projection of Saturn's southern aurora obtained with the FUV channel of UVIS on-board Cassini on DOY 21, 2009. Noon is to the bottom and dusk to the right. The grid shows latitudes at intervals of $10^{\circ}$ and meridians of $40^{\circ}$. Arrows indicate the main emission and the bifurcations of the main auroral emission. Adapted from Radioti et al. (2011b). (b) Polar projection of Saturn's aurora obtained with the HST on day 95,2013 . Noon is to the bottom and dusk to the right. A grey $10^{\circ} \times 10^{\circ}$ latitude-longitude grid is overlaid. Arrows show bursts of emissions indicative of reconnection of lobe flux in the magnetotail. Adapted from Nichols et al. (2014).

in arc and spot-like small-scale features. They are likely related to plasma flows enhanced from reconnection, which diverge into multiple narrow channels and then spread azimuthally and radially. The evolution of tail reconnection at Saturn may be illustrated by an ensemble of numerous narrow current wedges or inward transport initiated in the reconnection region, likely explained by multiple localized flow burst events.

Enhancements in ENA emission and Saturn kilometric radiation data, together with auroral observations from HST and UVIS, reported the initiation of several acceleration events in the midnight to dawn quadrant, at radial distances in the range from $15 R_{\mathrm{s}}$ to $20 R_{\mathrm{s}}$, related to tail reconnection (Mitchell et al. 2009). The formation of ENA emission is discussed in Section 2.1.2.2. Numerical simulations together with simultaneous UV and ENA emissions (Radioti et al. 2009a, 2013a) demonstrated that injected plasma populations can create auroral emissions at Saturn, by pitch angle diffusion associated with electron scattering by whistler-mode waves, while field-aligned currents driven by the pressure gradient along the boundaries of the cloud might have a smaller contribution.

Recently, UVIS auroral observations revealed the first and only observation of an Earth-like transpolar arc at Saturn (Fig. 7) (Radioti et al. 2014). At Earth, transpolar arcs are auroral features, which extend from the nightside auroral oval into the open magnetic field line region (polar cap) and they represent the optical signatures of magnetotail dynamics (e.g. Frank et al. 1982; Milan et al. 2005). The formation of the nightside polar arc at Saturn may be related to solar wind driven tail reconnection similarly to the terrestrial case (Milan et al. 2005). However, the rarity of the occurrence of the transpolar arc at Saturn indicates that the conditions for its formation are rarely met at the giant planet, contrary to the Earth.

\subsubsection{Space weather at the radiation belts}

The radiation belt environments of the outer planets are quite different from the one of the Earth, mainly due to the presence of moons and rings embedded in these systems. At Saturn, they were firstly explored using in-situ measurements from the

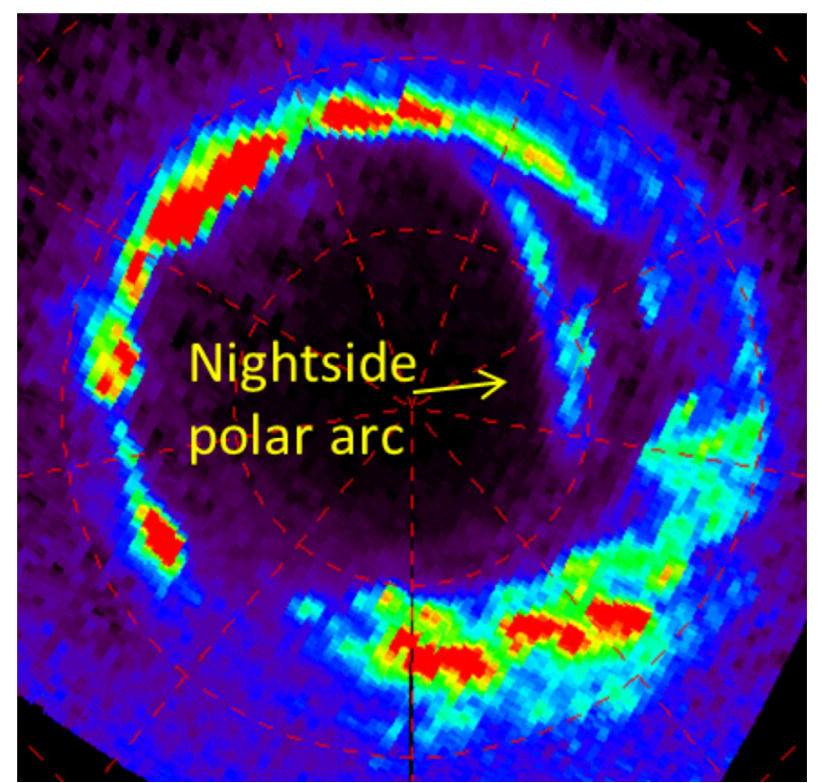

Fig. 7. Polar projection of Saturn's aurora as captured from UVIS on-board Cassini on DOY 224, 2008. Noon is to the bottom and dusk to the right. The arrow indicates the first observation of an Earth-like transpolar arc at Saturn. The formation of the nightside polar arc at Saturn may be related to tail reconnection. Adapted from Radioti et al. (2014).

Pioneer 11, Voyager 1 and 2 flyby missions (Van Allen 1983, 1984). The Cassini spacecraft arrived at Saturn in 2004 and it has since then been providing extensive data on the Kronian system and the radiation belts in particular.

The energy range of Saturn's charged particle population in the radiation belts is between some hundreds of $\mathrm{keV}$ up to tens of $\mathrm{MeV}$, while the radial extent of this magnetospheric region is between $2.3 R_{\mathrm{S}}$ up to $6 R_{\mathrm{S}}$ approximately (Gombosi et al. 2009; Kollmann et al. 2013). Figure 8 shows the typical phase space density profiles of protons in the Kronian radiation belts as a function of L-shell for several energy channels. Moving inside 2.3 $R_{\mathrm{s}}$, almost all the energetic particle population is 


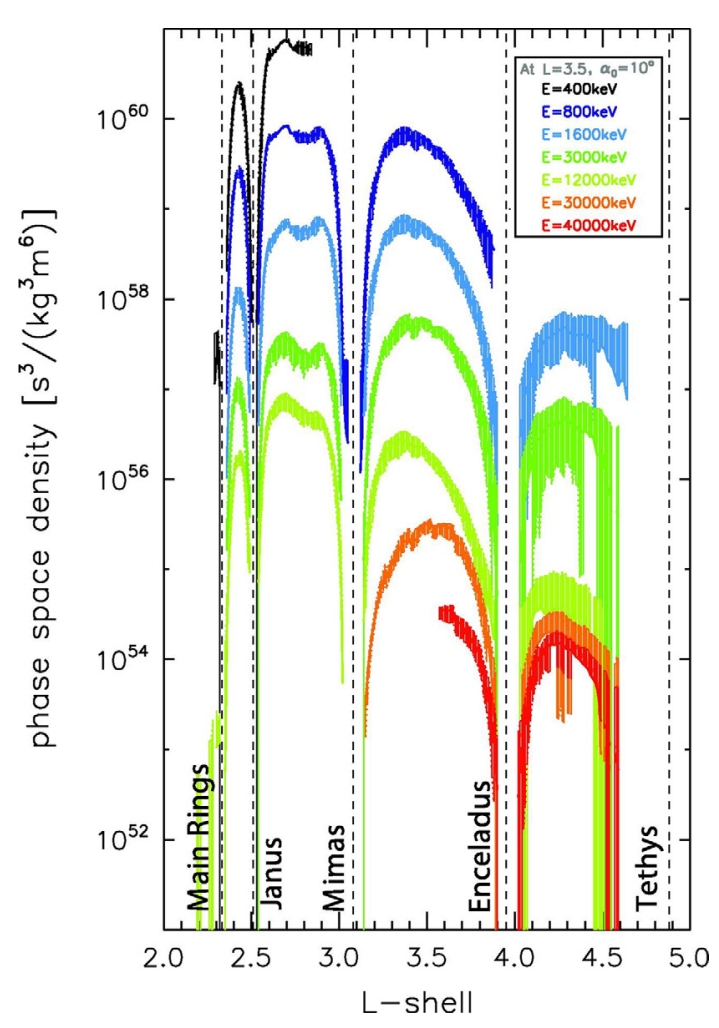

Fig. 8. Mission-averaged phase space density profiles of protons in Saturn's radiation belts. From Kollmann et al. (2013).

depleted due to the presence of the main rings. An exception may be the region inside the D-ring of Saturn, where, evidence of a tiny energetic particle trapping region has been reported (Krimigis et al. 2005). Its presence was inferred from the detection of an ENA population by the MIMI/INCA during the first Cassini orbit. The origin of this particle population is expected to be the main proton radiation belt, after the occurrence of double charge-exchange processes with particles of the Saturnian exosphere: these protons undergo a first charge-exchange, where they are transformed into planetdirected ENAs that can "overfly" the planetary rings, stripped off an electron upon entry in Saturn's exosphere and finally trapped as ions (Fig. 9).

The icy moons that inhabit the main radiation belts continuously absorb energetic particles at their orbits, separating in this way the ionic radiation belts and producing "sweeping corridors" (Kollmann et al. 2013; Kotova et al. 2015). Due to the approximate alignment of the magnetic axis with the rotation axis, the energetic particle losses due to the moons are expected to be much more pronounced than the ones in the other outer planets (Roussos et al. 2007). As a result, solar wind does not influence the inner radiation belt environment, which is rather stable. However, a new transient radiation belt was recently discovered near Dione's orbit, at radial distances from 4.89 to $8 R_{\mathrm{s}}$ approximately (Roussos et al. 2008). This transient belt can be observed for up to a few weeks or months and its occurrence is related to periods of enhanced solar activity. It can be considered therefore as an evident manifestation of space weather in the Saturnian system.

Another mechanism for the energetic particle loss in the Kronian radiation belts is the interaction of the ions with the moons' exospheres, and in particular with the exosphere and plumes of Enceladus (Tokar et al. 2006; Cassidy \& Johnson

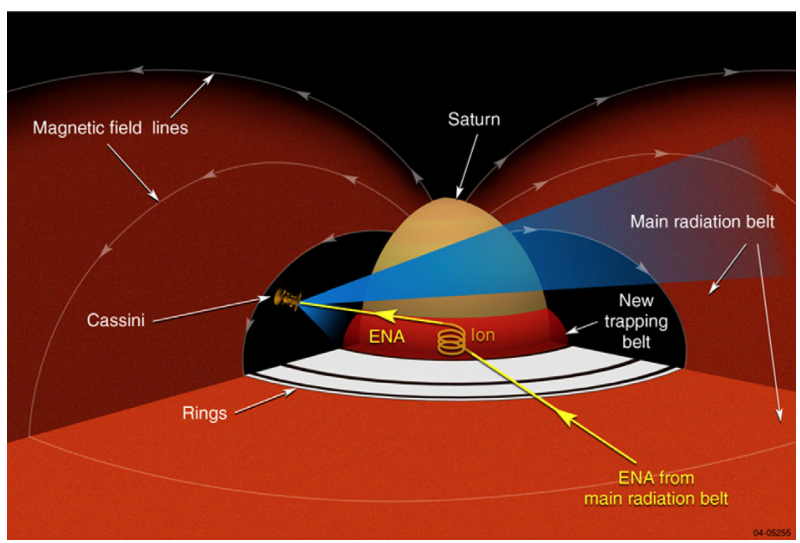

Fig. 9. Schematic of the charge-exchange/stripping process that begins as ENA emission from the main belt populates the innermost radiation belt ("new radiation belt") and ultimately produces ENA emission from Saturn's exosphere. From Krimigis et al. (2005).

2010). The most important source of the radiation belt protons with energies $>10 \mathrm{MeV}$ is the GCR population interacting with Saturn's atmosphere and rings through the CRAND process (Blake et al. 1983; Cooper 1983; Kollmann et al. 2013) (see also Sect. 2.1.1). Such a mechanism has an important role also at the Earth's radiation belts (Singer 1958). The CRAND process is also responsible for the most energetic component $(>10 \mathrm{MeV})$ of Saturn's innermost radiation belt (Kotova et al. 2014). While the proton radiation belts are characterized by stability, this is not the case for the electron radiation belts that are characterized by both complex temporal and spatial variations. Satellites orbiting within the magnetospheres of the giant planets are effective absorbers of trapped radiation, forming the characteristic and dominant structure of the radiation belts (Selesnick 1993) including electron macro and micro signatures. The solar UV irradiance from the thermosphere of Saturn and the solar wind are the most probable sources to account for the long-term variability of the electron radiation belts (Roussos et al. 2014), suggesting that external drivers play indeed an important role in Saturn's magnetospheric dynamics.

Several modelling studies have been performed to describe the electron radiation belts taking into account mainly the radial diffusion processes (Hood 1983, Santos-Costa et al. 2003). More recently, Lorenzato et al. (2012) adapted the Salammbô three-dimensional physical radiation belt model for Saturn's electron radiation belts (Santos-Costa et al. 2003). Apart from radial diffusion, this model considers other important physical processes governing radiation belt dynamics, and in particular, wave-particle interactions and losses due to the rings, the icy satellites and their exospheres (Lorenzato et al. 2012).

\subsubsection{Space weather at the Saturnian moons}

The plasma, magnetic field and neutral particles data obtained since the arrival of the Cassini spacecraft at Saturn in July 2004 have substantially enriched our knowledge of the magnetosphere-satellite interactions determining the space weather conditions at the giant planet's moons. In the following paragraphs we will focus on several of the moons of Saturn: Titan, the only known satellite with a dense nitrogen-dominated atmosphere, Rhea, Dione and Enceladus, a small icy moon identified as the major source of neutral particles and thus also as source of magnetospheric plasma. Titan is located within 
Saturn's magnetosphere for average solar wind conditions. The orbital velocities of these moons are exceeded by the speed of the (partially) corotating magnetospheric plasma. As a result, they are continuously "overtaken" by the magnetospheric plasma flow. The overall result of the interaction between plasma and satellite depends both on the plasma conditions, affected dynamically by internal plasma sources (e.g. neutral gas from Enceladus), and on the properties of each satellite itself (e.g. surface composition, endogenic neutral sources). Space weather conditions at the locations of these moons have been identified to some extent by the combination of interdisciplinary measurements with Cassini, however modelling and further investigation is necessary for resolving critical points to be taken into account during future missions.

Titan. Titan is the only other body, besides our own planet, to have a dense atmosphere composed essentially of molecular nitrogen $(\sim 97 \%)$, and hosting an active and complex organic chemistry created by the photolysis of methane $(\sim 2 \%$ in the stratosphere) and its interaction with nitrogen and hydrogen $(\sim 0.1 \%)$. Figure 10 shows the density profiles of the main components of Titan's upper atmosphere, as obtained from the Cassini Ion and Neutral Mass Spectrometer (INMS) measurements, averaged over all Cassini flybys. The reader is requested to refer to Cui et al. (2009b, Fig. 9), for a description of the observed diurnal variations of several representative ion species in Titan's ionosphere.

Adding to the Voyager 1 radio-occultation data, measurements by the Composite Infrared Radiometer Spectrometer (CIRS) on the orbiter and from the Huygens Atmospheric Structure Instrument (HASI) at the probe's landing site $\left(10^{\circ} \mathrm{S}, 192^{\circ} \mathrm{W}\right)$ from $1400 \mathrm{~km}$ in altitude down to the surface allowed us to reconstruct the temperature structure of Titan. A surface temperature of $93.65 \pm 0.25 \mathrm{~K}$ was measured for a pressure of $1467 \pm 1 \mathrm{mbar}$ (Fulchignoni et al. 2005). As revealed previously through Voyager data analysis, Titan's atmosphere is composed (from upper altitudes to lower ones) of an exosphere, a thermosphere, a mesosphere, a stratosphere and a troposphere, with two major temperature inversions at 40 and $250 \mathrm{~km}$, corresponding to the tropopause and stratopause, associated with temperatures of $70.43 \mathrm{~K}$ (min) and $186 \mathrm{~K}(\max )$. A mesopause was also found at $490 \mathrm{~km}$ (with $152 \mathrm{~K}$ ) in the early years of the Cassini mission, but has gradually disappeared in the recent years, leading to a more homogeneous vertical structure. The Voyager 1 UVS experiment had also recorded a temperature of $186 \pm 20 \mathrm{~K}$ at $1265 \mathrm{~km}$ during a solar occultation for a methane mixing ratio of $8 \pm 3 \%$ toward $1125 \mathrm{~km}$, placing the homopause level at around $925 \pm 70 \mathrm{~km}$.

The Cassini-Huygens mission demonstrated that Titan's atmosphere is a chemical factory in which the formation of complex positive and negative ions is initiated in the high thermosphere as a consequence of magnetospheric-ionosphericatmospheric interactions involving solar EUV, UV radiation, energetic ions and electrons (Waite et al. 2005). Indeed, besides the mother molecules, molecular nitrogen and methane, a diverse host of neutral components was discovered in the middle atmosphere, originating in the upper layers.

Table 1 (from Sittler et al. 2009) summarizes the main energy sources for Titan's upper atmosphere. As shown, energetic particles from Saturn's magnetosphere are, in addition to UV and EUV radiation, the most important energy

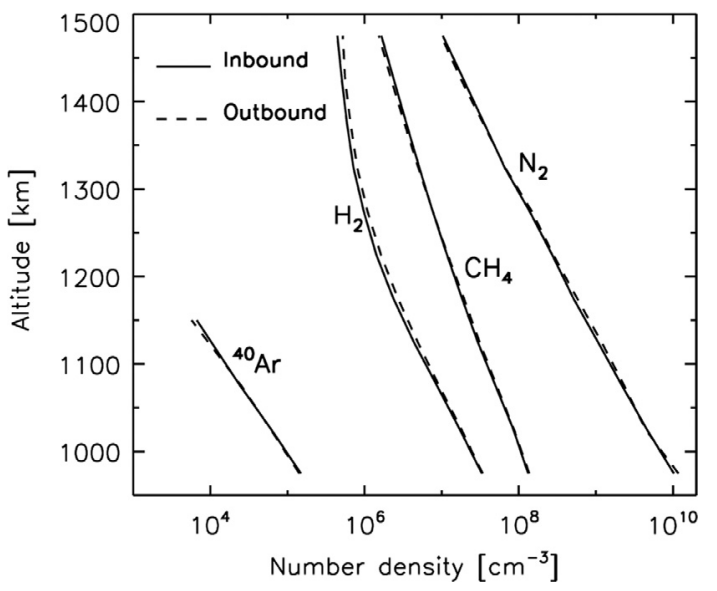

Fig. 10. The density profiles of $\mathrm{N}_{2}, \mathrm{CH}_{4}, \mathrm{H}_{2}$ and ${ }^{40} \mathrm{Ar}$, as obtained from the Cassini/INMS measurements, averaged over all Cassini flybys. The inbound (solid) and outbound (dashed) profiles are nearly identical, indicating that the wall effects are negligible for these species. From Cui et al. (2009a).

source for this complex chemical factory in Titan's upper atmosphere, and for creating an extended ionosphere.

Titan was found to have quite an extended ionosphere, between 700 and $2700 \mathrm{~km}$, essentially due to the lack of a strong intrinsic global magnetic field and the precipitation of energetic particles from Saturn's magnetosphere. At lower altitudes, galactic cosmic rays are responsible for the production of another ion layer in the atmosphere (between 40 and $140 \mathrm{~km}$ ), while the neutral atmospheric photochemistry is mainly driven by FUV solar photons.

Figure 11, adapted from Waite et al. (2004) and Sittler et al. (2009), shows schematically the main layers of Titan's upper atmosphere, and their interaction with the energetic plasma flow from Saturn's magnetosphere and the other energy sources. The direct analysis of the ionosphere by the INMS instrument during the low-altitude Cassini flybys of Titan shows the presence of many organic species at detectable levels, in spite of the very high altitude $(1100-1300 \mathrm{~km})$. The interpretation of INMS measurements (limited to masses up to 100 Daltons) and of Cassini/CAPS data (Coates et al. 2007, 2009) strongly suggests that high molecular weight species (up to several 1000 Daltons) may be present in the ionosphere (Waite et al. 2007, Fig. 7). Models applied to the data have pointed to the presence of complex molecules (see Tables 6.2 and 6.4 in Coustenis \& Taylor 2008). The vertical distributions of the trace gases increase with altitude, confirming that these species form in the upper atmosphere and then diffuse downward in the stratosphere. Among the trace species detected to date we find hydrocarbons $\left(\mathrm{C}_{2} \mathrm{H}_{2}, \mathrm{C}_{2} \mathrm{H}_{4}, \mathrm{C}_{2} \mathrm{H}_{6}\right.$, $\mathrm{C}_{3} \mathrm{H}_{4}, \mathrm{C}_{3} \mathrm{H}_{8}$, etc.) and nitriles $\left(\mathrm{HCN}, \mathrm{HC}_{3} \mathrm{~N}, \mathrm{C}_{2} \mathrm{~N}_{2}\right.$, etc.). Oxygen compounds such as $\mathrm{H}_{2} \mathrm{O}, \mathrm{CO}$ and $\mathrm{CO}_{2}$ have also been detected.

The Cassini-Huygens measurements have revolutionized our perception of the organic processes occurring in Titan's atmosphere, bringing forward a strong implication of the ionospheric chemistry in the formation of complex organic compounds in Titan's environment and acting as seed particles for the formation of tholin material and haze. These compounds are detectable in solar and stellar UV occultations and initiate the process of haze formation (Waite et al. 2007) to finally condense out starting at $\sim 950 \mathrm{~km}$ (Fig. 12). As the haze 
Table 1. Titan upper atmosphere energy sources. Adapted from Sittler et al. (2009).

\begin{tabular}{lccc}
\hline \hline Energy source & Energy flux $\left(\mathrm{erg} / \mathrm{cm}^{2} / \mathrm{s}\right)$ & Global input (Watts) & Comments \\
\hline Plasma protons & $1.6 \times 10^{-4}$ & $3.4 \times 10^{7}$ & Magnetized \\
Plasma electrons & $1.6 \times 10^{-4}$ & $3.4 \times 10^{7}$ & Magnetized \\
Plasma heavy ions & $1.5 \times 10^{-3}$ & $3.2 \times 10^{8}$ & Unmagnetized \\
Energetic ions & $5.0 \times 10^{-4}-1.0 \times 10^{-2}$ & $1.05 \times 10^{8}-2.0 \times 10^{9}$ & $27<$ Ep $<225 \mathrm{keV}$ \\
Energetic electrons & $2.0 \times 10^{-4}$ & $4.0 \times 10^{7}$ & $28<\mathrm{Ee}<533 \mathrm{keV}$ \\
UV airglow & $1.6 \times 10^{-3}$ & $3.5 \times 10^{8}$ & Altitude $\sim 1300 \mathrm{~km}$ \\
UV ionization & $1.6 \times 10^{-4}$ & $3.4 \times 10^{7}$ & Altitude $\sim 1300 \mathrm{~km}$ \\
GCR & $1.6 \times 10^{-4}-2.7 \times 10^{-3}$ & $3.2 \times 10^{7}-5.4 \times 10^{8}$ & \\
Dust & $1.8 \times 10^{-3}$ & $1.8 \times 10^{8}$ & Interplanetary dust \\
\hline
\end{tabular}

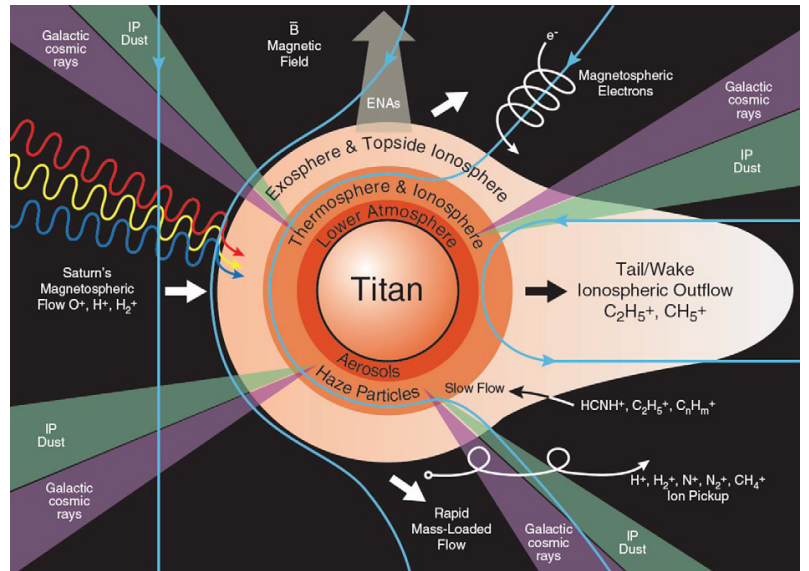

Fig. 11. The main layers of Titan's upper atmosphere, and its illumination by solar UV (blue rays), solar visible light (yellow rays) and solar infrared light (red rays). The energy input from Saturn's magnetospheric interaction shows a strong interaction with Titan's atmosphere and the corresponding induced magnetosphere with draped magnetic field lines. The other energy sources (galactic cosmic rays, interplanetary dust) are also schematically shown. Adapted from Waite et al. (2004) and Sittler et al. (2009).

particles fall through the atmosphere and grow, they become detectable with imaging systems such as the Cassini/Imaging SubSystem (ISS) at $\sim 500 \mathrm{~km}$ altitude and are ubiquitous throughout the stratosphere (Porco et al. 2005). They are strong absorbers of solar UV and visible radiation and play a fundamental role in heating Titan's stratosphere and driving wind systems in the middle atmosphere, much as ozone does in the Earth's middle atmosphere.

Both the Voyager and the Cassini data indicated the presence of temperature variations with latitude also with the coldest temperatures in the past 10 years recorded by Cassini first in the Northern regions associated with enhanced gas concentration and haze opacity (as this may be caused by more efficient cooling) or/and dynamical inertia at a time in the beginning of the mission (Bampasidis et al. 2012; Coustenis et al. 2015) when it was early southern summer on Titan to well after the northern spring equinox (in August 2009) and now into southern winter, with very cold temperatures and dramatically enhanced chemical composition found there now. In mid-2010 the epoch on Titan was the same as during the V1 encounter, almost 30 years before (1 Titan year, Coustenis et al. 2015). No significant changes in temperature were reported for the inter-annual time lapse, but extreme changes depending on the season were found, essentially at the poles, with the southern pole being currently extremely

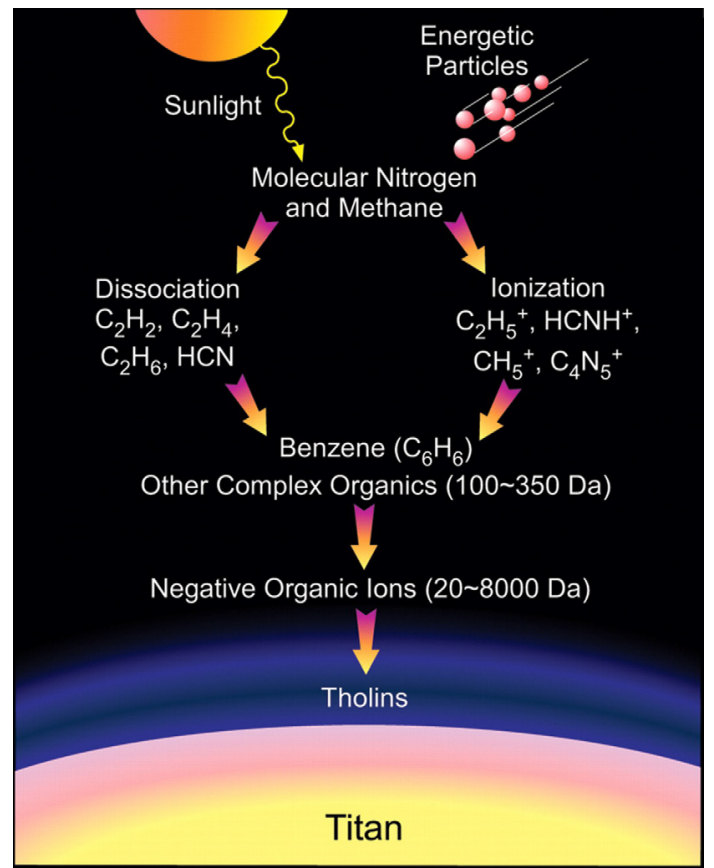

Fig. 12. Schematic of Titan's complex organic chemistry. Adapted from Waite et al. (2007).

cold and having reached that state in a short time (within a couple of years, Coustenis et al. 2016).

Since Titan is mostly inside Saturn's magnetosphere (e.g. Dandouras et al. 2009; Garnier et al. 2010), but can also get under some conditions into the magnetosheath (Garnier et al. 2009) or even in the solar wind (Bertucci et al. 2014), particle precipitation, hence ionization, can be very variable depending on the position of the satellite and on the local plasma conditions. Knowledge of the whole ionization profile in the atmosphere of Titan is however widespread. In the following paragraphs, we examine some of the salient features.

Energetic plasma from Saturn's magnetosphere (and under some conditions directly from the solar wind), solar EUV-XUV radiation and cosmic rays are the main sources of the ionization of the neutral gas in Titan's atmosphere (see also Table 1). Each source has a main ionization altitude that creates several ionospheric layers above $50 \mathrm{~km}$ (Hamelin et al. 2007; Cravens et al. 2008, 2009; López-Moreno et al. 2008, and references therein). The ion production rates in Titan's ionosphere, for the dayside (Richard et al. 2015a and references therein) and nightside (Richard et al. 2015b and references therein), have been extensively studied. During the daytime, the electron density varies between 3000 and $7000 \mathrm{~cm}^{-3}$ for a zenith angle 
of $0^{\circ}$, with a maximum at about $1050 \mathrm{~km}$. Both parameter variations with the zenith angle follow basically a Chapman law (Lilensten et al. 2005a). The major ions produced are $\mathrm{N}_{2}{ }^{+}, \mathrm{N}^{+}$and $\mathrm{CH}_{4}{ }^{+}$. Minor ions include $\mathrm{CH}_{3}{ }^{+}$and other minor $\mathrm{CH}_{4}$ ionization products. Vigren et al. (2013) used in-situ $\mathrm{N}_{2}$ and $\mathrm{CH}_{4}$ measurements by the Ion Neutral Mass Spectrometer (INMS) in order to determine (through modelling) the electron production rates in Titan's upper atmosphere. Finally, Titan's ionosphere also has a trace of doubly charged $\mathrm{N}_{2}{ }^{++}$ions (Lilensten et al. 2005b).

The particles precipitating in the atmosphere of Titan are of different origin and therefore of different energies. The less energetic ones are the protons and electrons of the solar wind, but exposure of Titan to the solar wind can occur only under high solar wind dynamic pressure conditions and if, at that time, Titan is in the subsolar part of its orbit (Bertucci et al. 2014) their typical energy is of a few keVs. They create an upper ionization layer at about $900 \mathrm{~km}$ and up to $1300 \mathrm{~km}$. Its vertical profile can be compared to that in the ionosphere of the Earth, with a similar F region (Galand et al. 1999). However, the most important interaction of Titan's upper atmosphere is with the energetic protons, heavy ions and electrons from Saturn's magnetosphere, which results in an energy input of the order of $10^{8}-10^{9} \mathrm{~W}$ (Table 1). It creates additional features known as electron bite-outs (Snowden \& Yelle 2014).

Three layers of detached haze can be seen in the atmosphere of Titan. Their origin lies in ion-neutral chemistry. The neutrals originate from the atmosphere. The electrons and ions are linked to three separate sources, all-dependent on space weather. Solar wind electrons create ionization around $900 \mathrm{~km}$, protons precipitating from Saturn create the layer at $500 \mathrm{~km}$ and cosmic rays are responsible for the layer at $65 \mathrm{~km}$ (Gronoff et al. 2009a, 2009b). However, the source is only part of the story of the layers, which have their own seasonal latitudinal variability (Larson et al. 2015). It seems that Titan is a unique laboratory for studying the effects (and interactions) between the three layers and in general the formation of haze and its interaction with the environment.

Titan's interaction with the solar wind is in many ways similar to that of unmagnetized planets like Mars and Venus (see also Sect. 2.2.1) and of active comets despite the different plasma properties in the outer Solar System (Bertucci et al. 2015). Therefore, Titan constitutes a space weather laboratory, providing a set of different configurations, determined by the moon's position in the Kronian magnetosphere and with respect to the Sun.

The Rhea and Dione icy moons. Oxygen and carbon-dioxide exospheres at Rhea and Dione were discovered by the Cassini INMS and CAPS instruments, during the north and south Rhea flybys (respectively, on March 2, 2010 and January 11, 2011) (Teolis et al. 2010), and during the equatorial Dione flyby, on December 12, 2011 (Teolis et al. 2010; Teolis \& Waite 2012). During the recent Dione north polar flyby on June 16, 2015, INMS has also detected $\mathrm{O}_{2}$ (Teolis \& Waite 2016). The $\mathrm{O}_{2}$ and $\mathrm{CO}_{2}$ densities at the time of closest approach to Dione were roughly equal to $\sim 2 \times 10^{10} \mathrm{~m}^{-3}$. These Cassini discoveries, together with the HST (Hall et al. 1995) and the Galileo spacecraft UV investigation (Hansen et al. 2005) observing an $\mathrm{O}_{2}$ exosphere around Jupiter's icy satellite Europa, suggest that the exosphere generation mechanisms could be common among the different icy moons in the outer
Solar System. Variabilities in these neutral environments due to interactions with the parent planet's magnetospheric environment result in the determination of space weather conditions in the near-satellite environment, indicative of the characteristic processes active at different orbital phases.

The exospheric density distributions around Rhea and Dione icy moons are closely related to their surface composition but the exact connection is still not clear. In general, Saturn's icy moon surfaces are dominated by water ice in crystalline form, in particular across Enceladus' south pole where the "tiger stripes" features are observed (Brown et al. 2006; Jaumann et al. 2008). $\mathrm{CO}_{2}$ is detected on Hyperion (Cruikshank et al. 2010), Iapetus (Cruikshank et al. 2008; Pinilla-Alonso et al. 2011; Clark et al. 2012), Phoebe (Clark et al. 2005; Buratti et al. 2008; Coradini et al. 2008), Tethys, Dione, Mimas and Rhea as well (Clark et al. 2008). The composition and distribution of the exogenic dark material dispersed on Dione's trailing hemisphere is discussed in Clark et al. (2008) and Stephan et al. (2010), while a detailed study of Rhea's properties is done by Stephan et al. (2012). Although so far several laboratory studies on the efficiency of the neutral particle release from ice bombarded by energetic particles have been done (see Famà et al. 2008 and references therein) the actual conditions in space can be very different from those in the laboratory. In this frame, measurements of the released neutrals above Rhea and Dione and their correlation with the surface composition data obtained by other instruments become very important: they can provide information on the way contaminants affect the exosphere composition and spatial distribution, determining the space weather conditions around the moons.

Although the existence of an $\mathrm{O}_{2}$ exosphere around Rhea and Dione has been recently explained on the basis of $\mathrm{H}_{2} \mathrm{O}$ ice radiolysis by Saturn's magnetospheric ions and subsequent sputtering of the dissociation products and their combinations (Teolis et al. 2010; Teolis \& Waite 2012), the origin of $\mathrm{CO}_{2}$ gas in both cases is still an open question. Exospheric $\mathrm{CO}_{2}$ (i) may be synthesized from radiolysis involving surface-bound oxygen and endogenic and/or implanted organics, and/or (ii) may be due to escape of primordial $\mathrm{CO}_{2}$ from the ice (Teolis \& Waite 2012). Moreover, the Cassini $\mathrm{CO}_{2}$ measurements pose an additional puzzling question because recent studies based on the Cassini Visible and Infrared Mapping Spectrometer (VIMS) data can find no evidence for a currently active endogenic $\mathrm{CO}_{2}$ source either at Dione (Stephan et al. 2012) or at Rhea (Stephan et al. 2010). Would then the presence of an exospheric $\mathrm{CO}_{2}$ abundance similar to that of $\mathrm{O}_{2}$ on these moons be consistent with a radiolysis scenario taking place upon a surface consisting mostly of $\mathrm{H}_{2} \mathrm{O}$ ice? Such a process would have a strong dependence on the impacting plasma conditions in the near-moon space environment and their variability. Teolis \& Waite (2016) estimated that the $\mathrm{O}_{2}$ source rates at Dione (Rhea), equal to $\sim 45(7) \times 10^{21} \mathrm{~s}^{-1}$, are $\sim 50(300)$ times lower than those expected from known $\mathrm{O}_{2}$ radiolysis yields from ion-irradiated pure water ice, measured in the laboratory. This finding rules out ion sputtering as a major exospheric source and implies a nanometre-scale surface refractory lag layer, consisting of concentrated carbonaceous impurities (Teolis \& Waite 2012); impact deposition, gardening and vaporization may control the global $\mathrm{O}_{2}$ source rates by fresh $\mathrm{H}_{2} \mathrm{O}$ ice exposure to surface radiolysis and trapped oxidant ejection. 
Enceladus. The plasma interaction of Saturn's icy moon Enceladus generates a hemisphere coupling current system that directly connects the giant planet's northern and southern polar magnetospheres. Several studies based on the Cassini/ Magnetometer (MAG) data from close Enceladus flybys suggest that the outgassing from the moon's interior exhibits time variability (e.g. Saur et al. 2008; Smith et al. 2010). The neutral gas in the Enceladus plume gets partially ionized due to charge exchange with the incident magnetospheric plasma and, to a minor degree, due to solar ultraviolet (UV) radiation and electron impact ionization. Simon et al. (2013a) predicted that changes in both the neutral gas and the dust component of the plume should lead to observable changes in the footprint brightness. To do this, these authors employed the energy flux radiated away by Enceladus' magnetospheric interaction as a measure of the footprint brightness in Saturn's polar ionosphere. A positive correlation between the energy transmitted from the Enceladus interaction region to each hemisphere of Saturn and the corresponding footprint brightness was assumed. Energy transport processes were not taken into account; instead, a sophisticated description of the energy generation in the vicinity of Enceladus was implemented. The findings by Simon et al. (2013a) clearly show that any variation in the endogenic activity of the moon can provoke equivalently space weather activity not only in the Saturnian magnetosphere in the vicinity of the moon, but also in the regions of the planet's ionosphere near the footprint. In the vicinity of the moon, since both the incident magnetospheric plasma and the neutrals in the Enceladus plume consist of water group particles, the charge-exchange process does not alter the total mass of the charged particles in the interaction region, but it only drains momentum from the plasma (Khurana et al. 2007; Kriegel et al. 2009). Simon et al. (2014) proposed that such continuities attributed to the Alfvenic nature of Enceladus plasma may also occur at the Jovian moon Europa where the HST recently observed a transient south-polar plume of water vapour (Roth et al. 2014a).

\subsubsection{Space weather in the Jovian system}

The magnetic moment of Jupiter's magnetic field is 20,000 times larger than the Earth's. As a result, Jupiter's magnetosphere extends to about $150 R_{\mathrm{J}}\left(R_{\mathrm{J}}=71,492 \mathrm{~km}\right)$ and its radiation belts are the strongest ones in the Solar System. The four major moons of Jupiter lie deep within the Jovian magnetopause. Io, with its strong volcanic activity and its sulphur dioxide atmosphere, injects constantly particles into the Jovian magnetosphere, in the form of either gas or plasma. The plasma and neutral source rate variability in the Jovian system, determined strongly by Io's volcanic activity, is expected to have a direct effect on the plasma interactions with Jupiter's ionosphere, the radiation belts, the lunar exospheres and the rings. Solar wind variability at the distance of Jupiter also plays a role in these interactions (Bunce et al. 2004). Therefore, identifying the characteristics of the interaction processes within the Jupiter system and distinguishing their spatial properties will set the basis for a better understanding of the space weather conditions around the giant planet.

Whereas Io, Europa and Ganymede are located in the inner magnetosphere of Jupiter ( $\lesssim 20 R_{\mathrm{J}}$ ), where the magnetic energy dominates the energy density, Callisto's orbit at $25 R_{\mathrm{J}}$ lies in the middle magnetosphere where a significant portion of the energy density resides in the thermal plasma and energetic particles that rotate around Jupiter. External currents produce a disk-like magnetic field structure. The interactions between the Galilean satellites and the plasma of the Jovian magnetosphere lead to local changes in the charged particle populations through either additions of new ions or through changes of the momentum that produce plasma heating or cooling. Plasma-surface interactions at Europa and Ganymede have been shown to generate neutral environments around these moons through either direct ion sputtering or radiolysis, followed by sputtering of the recombined water-dissociation products (Shematovich \& Johnson 2001; Shematovich et al. 2005; Cassidy et al. 2010, 2013). These exospheres have been demonstrated to be spatially non-uniform (Plainaki et al. 2012, $2013,2015)$, depending both on the moons' illumination by the Sun and on Jupiter's magnetospheric plasma properties. It is clear, therefore, that plasma and energetic ion dynamics at the near-Europa and near-Ganymede space result in space weather phenomena at different timescales, with effects on the morphology of the neutral environment around these icy moons.

\subsubsection{Solar wind effects on the aurora}

Jupiter's internal plasma sources (Io volcanism) and fast rotation are the main drivers of auroral dynamics, contrary to the solar wind driven terrestrial aurora. According to Grodent (2015), the aurora at Jupiter may be divided into four components (Fig. 13): the main emission (i.e. Grodent et al. $2003 \mathrm{~b}$ ), the emissions located equatorward of the main emission (i.e. Radioti et al. 2009b), the polar emissions (Grodent et al. 2003a; Stallard et al. 2003) and the satellite footprints (Clarke et al. 2002; Bonfond et al. 2008; Grodent et al. 2009). Each of these auroral components corresponds to different magnetospheric regions and is generated by different processes and thus not all of them respond to solar windmagnetosphere interaction.

Theoretical studies suggested that the main auroral emission at Jupiter is related to internal processes and in particular to the magnetosphere-ionosphere coupling current system associated with the breakdown of rigid corotation in the middle magnetosphere (Cowley \& Bunce 2001; Hill 2001). The main constituents of Jupiter's ionosphere are $\mathrm{H}_{3}{ }^{+}$ and $\mathrm{H}^{+}$(see Fig. 14). First-order models predicted that solar wind compression regions would induce an increase in the angular velocity of the equatorial plasma and decrease the currents related to the lag from corotation, thus resulting in a dimmer aurora (e.g. Southwood \& Kivelson 2001). Radioti et al. (2008a) showed that the main emission exhibits a persistent dim region (discontinuity) in the pre-noon sector (Fig. 13), which is suggested to represent a decrease in field-aligned current intensity possibly related to solar wind driven magnetospheric convection. This discontinuity in the main emission was further predicted by MHD simulations of the Jovian magnetosphere (Chané et al. 2013).

The high-latitude auroras inside the main emission (polar emissions, see Fig. 13) are extremely variable and may be magnetically connected to the middle and outer magnetosphere and possibly related to a sector of the Dungey and/ or Vasyliunas cycle flows (Cowley \& Bunce 2003; Grodent et al. 2003a; Stallard et al. 2003). A few regions in the polar aurora may be related to solar wind interactions in the dayside. An extremely bright flare (up to $10 \mathrm{MR}$ ) was observed close to magnetic noon, lasting a few minutes (Waite et al. 2001). Bonfond et al. (2011) reported two cases of 


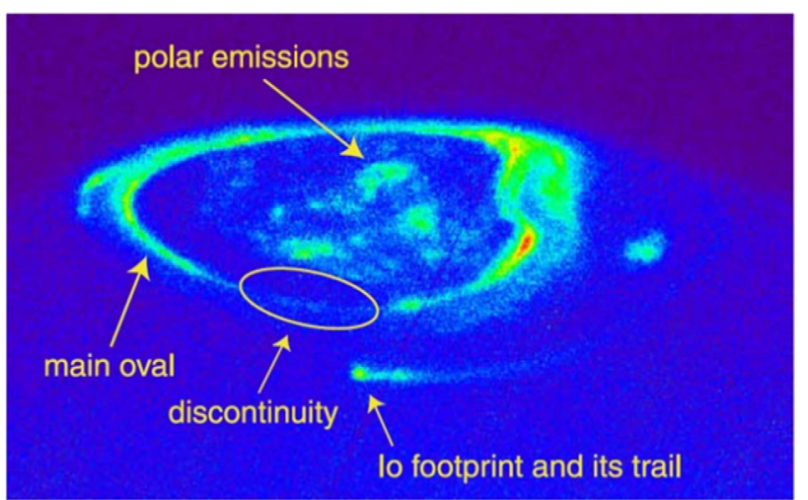

Fig. 13. HST-STIS image showing the FUV auroral emission at the north pole of Jupiter, taken on 28 December 2000. The arrows indicate the main auroral features: the main emission, the Io footprint and its trail and the polar emissions. The ellipse indicates the discontinuity in the main emission. From Radioti et al. (2008a).

quasiperiodic variations of flare emissions, with a reoccurrence rate of 2-3 minutes and noted a rapid downward propagation of the flares in one of the cases. They tentatively attributed these periodic flares to a signature of pulsed component reconnection on the dayside magnetosphere. Bunce et al. (2004) showed that periodic pulsed reconnection during intervals of strong solar wind interaction could excite high-latitude UV and X-ray emission (Gladstone et al. 2002; Elsner et al. 2005).

Occasionally, HST observed isolated spots in the polar region (high-latitude aurora) in the pre-noon and dusk region (Grodent et al. 2003a, 2004; Radioti et al. 2008b, 2010, 2011a) and related them to inward flow release from tail reconnection. While in some studies, due to their periodic occurrence, the spots were attributed to internally driven reconnection (e.g. Radioti et al. 2008b), for some others the origin of the reconnection (solar wind or internally driven) was uncertain (Grodent et al. 2004; Radioti et al. 2011a). Finally, quasi-sun-aligned polar filaments have also been reported (Nichols et al. 2009b). They were morphologically similar to the terrestrial transpolar arcs, which may be related to solar wind tail dynamics (Milan et al. 2005); nevertheless, the Jovian arcs are possibly not generated in the same manner.

Several studies provided evidence of the correlation of the solar wind pressure with the Jovian auroral emissions. The first evidence came from observations of the planet's $\mathrm{H}_{3}{ }^{+}$emissions (NASA IRTF) combined with solar wind data (Ulysses), which showed a correlation between the change in solar wind dynamic pressure between their auroral observations and the total intensity of the $\mathrm{H}_{3}^{+}$auroral emission (Baron et al. 1996). Later on, several studies reported a correlation of the auroral emitted power with the solar wind pressure (Nichols et al. 2007; Clarke et al. 2009; Nichols et al. 2009a). Based on images acquired in 2000 , while Cassini was upstream of Jupiter, Nichols et al. (2007) reported enhanced auroras associated with a compression region (Fig. 15), in contrast with theoretical predictions (Southwood \& Kivelson 2001). In a follow-up study, Clarke et al. (2009) compared the brightness of the whole Jovian aurora with solar wind conditions during the large 2007 HST campaign and concluded on a correlation of the auroral brightness with the solar wind pressure. Nichols et al. (2009a) considered individual components of auroral emissions (the low-latitude emissions, the main emissions and the high-latitude emissions) and showed that the outer

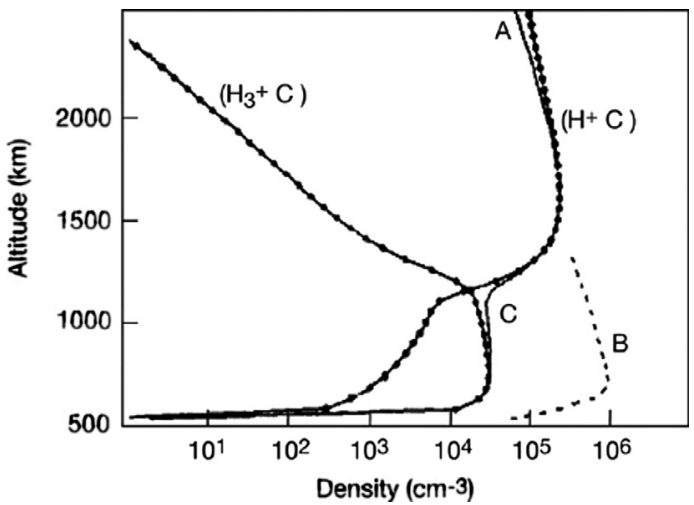

Fig. 14. Measured (curve A) and modelled density profiles of various constituents of Jupiter's ionosphere. From Majeed \& McConnell (1991).

regions do not appear to be correlated with the solar wind, but enhancements of the main emissions and partially the polar emissions are associated with solar wind compression regions, as in Nichols et al. (2007).

\subsubsection{Space weather at the radiation belts}

Jupiter has the most intense radiation belts in our Solar System, already discovered in the $1950 \mathrm{~s}$ through radio emission measurements from the ground (Burke \& Franklin 1955; Bolton et al. 2004). Both the energetic electron and proton fluxes show an increase with decreasing radial distance, with a peak observed near the orbit of the moon Amalthea $(L \sim 2.54)$ and a secondary peak at a distance of $L \sim 1.5$ with energies up to $50 \mathrm{MeV}$ approximately (Bolton et al. 2002). Satellites also inhabit the inner Jovian magnetosphere, leading to particle losses. However, due to the fact that Jupiter has a dipole tilt of approximately $10^{\circ}$, these losses are not as pronounced as in the Saturnian case (Santos-Costa \& Bourdarie 2001; Lilensten et al. 2014), nevertheless, they are still very important.

A thin ring system is present in the Jovian environment, extending to distances in the range $1.3<L<3.1$. It is mainly composed of dust particles with sizes $0.1-100 \mu \mathrm{m}$, which are not large enough to cause significant particle losses like the ones due to the moons (Showalter et al. 1987; Ockert-Bell et al. 1999; Bolton et al. 2004). Nevertheless, the dust-plasma interaction definitely modifies the radiation belt dynamics.

Energy losses of particles caused by synchrotron emission, in combination with a very extended Jovian atmosphere, lead to significant atmospheric losses (Abel \& Thorne 2003). In particular, losses due to synchrotron emission are considered to be the most significant ones in the innermost part of the Jovian radiation belts, $L<1.8$ (Bolton et al. 2004). Similar to the Earth's case, radial diffusion plays a significant role in determining the dynamics of the Jovian radiation belts. However, the most widely accepted radial diffusion mechanism is not related to nightside reconnection processes, as for the Earth's radiation belts, but to winds originating from the planet's ionosphere (Brice \& McDonough 1973; Schardt \& Goertz 1983). Both short- and long-term variations have been reported to exist in Jupiter's radiation belts. Correlations found with solar wind variations (Bolton et al. 1989) and solar F10.7 flux enhancements (Miyoshi et al. 1999) have been proposed to be related to the long- and short-term variations, accordingly. Finally, wave-particle interactions also constitute 

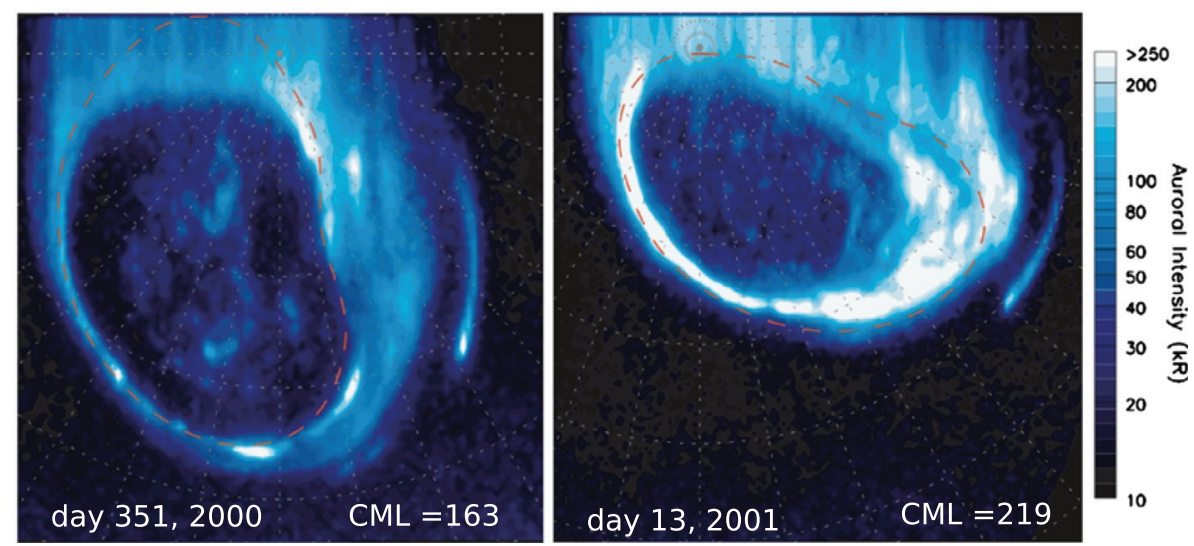

Fig. 15. Two representative polar projections of Jupiter's northern UV aurora obtained by the HST at different CMLS on day 351 , in 2000 and on day 13, in 2001, respectively. The CML of each image is aligned toward the bottom of each image, such that dawn is to the left, dusk to the right, and midnight to the top. The intensity scale is logarithmic and saturated at $250 \mathrm{kR}$. The red dashed line indicates the statistical reference oval from Grodent et al. (2003a). A $10^{\circ} \times 10^{\circ}$ jovigraphic grid is overlaid. The auroras correspond to rarefaction and compression of solar wind conditions. Adapted from Nichols et al. (2007).

both source and loss acceleration mechanisms in the system. They rather seem to be more effective in the outermost part of the belts, outside the orbit of Io $(L \sim 5.9)$, and only play a minor role in the innermost part (Bolton et al. 2004; Woodfield et al. 2014).

Since the Jovian radiation belt environment is considered to be one of the most intense and hazardous environments in our Solar System, several models to describe it have been developed (e.g. Santos-Costa et al. 2001; Santos-Costa \& Bourdarie 2001; Sicard-Piet et al. 2011).

\subsubsection{Space weather at the Galilean moons}

Io's atmosphere is not homogeneous, showing increased density near the volcanic plumes and possibly above other hot spots on the surface. Io's lower latitudes are partially shielded by the presence of a $\mathrm{SO}_{2}$ vapour-pressure controlled atmosphere, which is much less dense at the relatively cold poles. Based on laboratory work (Moore 1984), Wong and Johnson (1996) suggested that Io's poles have been darkened by plasma flux. The icy moons Europa and Ganymede possess tenuous atmospheres, often referred to as exospheres, dominated, near the surface, by $\mathrm{O}_{2}$. The $\mathrm{O}_{2}$ species has been observed indirectly through UV measurements of atomic oxygen de-excitation attributed mainly to electron-impact dissociative excitation of a molecular oxygen exosphere (Hall et al. 1995, 1998; Saur et al. 2011; McGrath et al. 2013; Roth et al. 2014a). At Europa and Ganymede, the Jovian magnetospheric plasma confined by Jupiter's magnetic field slightly subcorotates, with plasma flow velocities that are much greater than the orbital velocity of these moons (in anticlockwise direction, if seen from the North). As a result, the bulk plasma flow is constantly overtaking these satellites. The ions of the Jovian magnetosphere impacting the moon surfaces release material via direct ion sputtering and radiolysis (Johnson 2001). Considering that water ice is the major surface component, the generated exosphere is expected to be a mixture of $\mathrm{H}_{2} \mathrm{O}, \mathrm{O}_{2}$ and $\mathrm{H}_{2}$ and of some other water products, such as $\mathrm{OH}$ and $\mathrm{O}$ (Shematovich et al. 2005; Smyth \& Marconi 2006; Coustenis et al. 2010; Dalton et al. 2010; Plainaki et al. 2012). Exospheric $\mathrm{H}_{2} \mathrm{O}$ and $\mathrm{H}_{2}$ are expected to dominate at higher altitudes, except in a constrained region above the moons' subsolar point where sublimated water can locally have increased densities (Plainaki et al. 2012, 2015). Some minor species (e.g. Na) are also expected to exist in these exospheric environments (Leblanc et al. 2005; Cassidy et al. 2008). Charge-exchange collisions between magnetospheric plasma and the tenuous atmospheres of the icy moons result in the production of neutral particles that are subsequently set in orbit around Jupiter, forming the moons' neutral gas clouds. Inside these neutral gas clouds, as well as inside the actual moon exospheres, ionization processes (due to either photons or electrons) and charge-exchange collisions (due to the mangetospheric plasma) create plasma sources for the Jovian magnetosphere; once these particles are charged, they are accelerated by the Lorentz force and start to corotate around Jupiter. The total ionospheric outflow at Ganymede estimated on the basis of Galileo PLS measurements (Frank et al. 1997) was found to be $\sim 2$ orders of magnitude higher than the pick-up rate of newly ionized particles from the moon's extended exosphere, as observed by the Galileo magnetometer during two upstream flybys of Ganymede (Volwerk et al. 2013). Variability in the flux of the magnetospheric plasma of iogenic origin, therefore, results in a series of space weather effects in the whole system, with impacts on the actual neutral environment's spatial and energy distributions around the moons, as well as on the supply to the magnetosphere of charged particle of lunar origin.

Variations in the charged population properties due to variability in Io's volcanic activity and/or the moon's orbital position with respect to Jupiter's plasma sheet (JPS) could result in different surface precipitation patterns for the ions impacting Ganymede (see Fig. 16). As a consequence, space weather phenomena at Ganymede are expected to manifest themselves through variations (a) in the location of the OCFB boundary and consequently in the plasma precipitation patterns and (b) in the plasma-neutral interactions determining the exosphere spatial distribution. In fact, the intrinsic magnetic field of Ganymede, reconnecting with the external Jovian magnetic field, partially shields the surface from the ion impact, especially at the equatorial latitudes (e.g. Kivelson et al. 1997). The scale height of the plasma sheet at the distance of Ganymede, cantered roughly around the Jovian magnetic equator, is low (Khurana et al. 2004, 2007). Since Jupiter's magnetic axis is tilted by $10^{\circ}$ with respect to its rotational axis, the plasma sheet oscillates up and down the satellite (McGrath et al. 2013). Above about $10 \mathrm{keV}$ the ion 
(a)

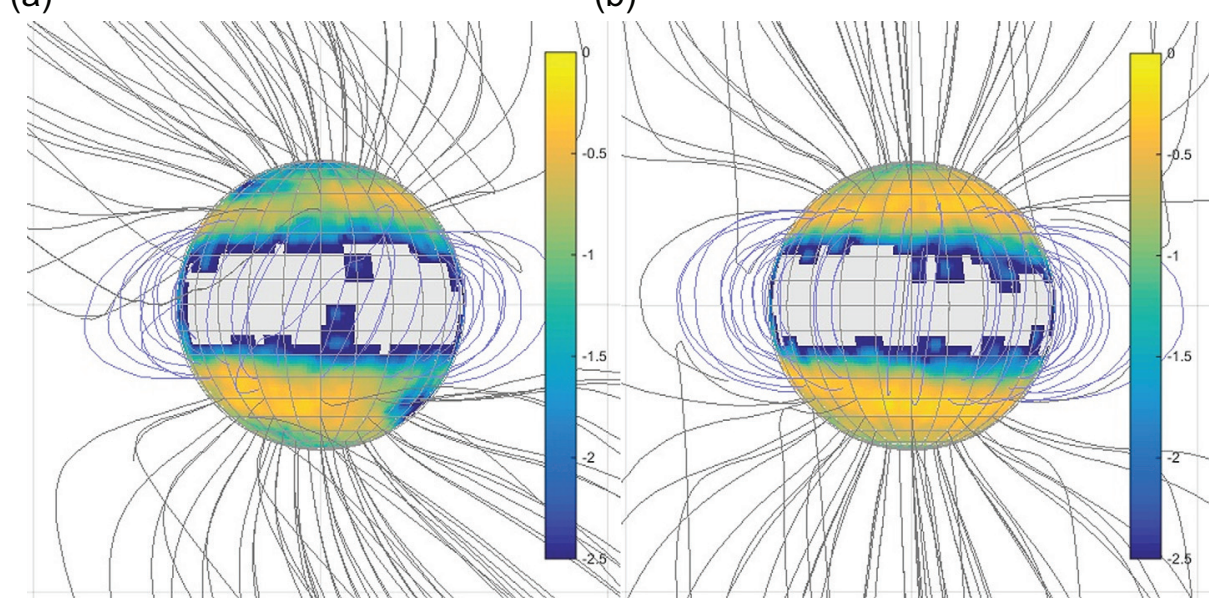

Fig. 16. Normalized flux (in logarithmic scale) of $100 \mathrm{keV} \mathrm{O}^{+}$impacting Ganymede's surface for two different configurations: (a) Ganymede is under Jupiter's plasma sheet and (b) Ganymede is in the centre of Jupiter's plasma sheet. The magnetic field configurations in each case are given by the Jia et al. (2009) model and they are consistent with the G8 (a) and G2 (b) Galileo flybys.

flux falls off with increasing energy (Paranicas et al. 1999). However, the charged energetic particle populations play a significant role in the magnetosphere-moons interactions (Mauk et al. 1996), having distributions that vary in both long (depending on the moon's orbit) and short (depending on the iogenic plasma properties) terms. The highest ion precipitation rate, leading to intense sputtering and radiolysis effects, is expected to occur near the OCFB regions (e.g. Kivelson et al. 1997). The dynamics of the Jovian magnetospheric plasma controls the dynamics of plasma entry and circulation in Ganymede's magnetosphere, primarily through reconnection between Jupiter's and Ganymede's magnetic fields (Jia et al. 2010). Eventually, it determines the ion precipitation toward the moon's surface (Johnson 1997). Plasma variations, in both density and speed direction, affect also the exosphere generation rates, determining the actual supply of surfacereleased material to the neutral environment as well as the spatial distribution of the latter. It has been shown that the spatial distribution of an icy moon exosphere strongly depends on the illumination of the moon, since the surface temperature is responsible for the efficiency of radiolysis (Plainaki et al. 2012) as well as for the sublimation rate (Smyth \& Marconi 2006). In an analogous way, the variability in the plasma electron density distribution around the moon during different orbital phases and/or different periods of plasma injections from Io affects the exosphere loss rates and determines its actual morphology. The moon exosphere source-loss balance, consequently, depends on a complex pattern of planetary space weather conditions. Plainaki et al. (2015) used previously published estimates of plasma parameters (McNutt et al. 1981; Scudder et al. 1981; Gurnett et al. 1996; Eviatar et al. 2001; Kivelson et al. 2004) to calculate the rates of the most important plasma-moon interactions leading to the loss of Ganymede's exosphere. They found that the loss rate for $\mathrm{H}_{2} \mathrm{O}$ in the polar caps is due to its charge exchange with ionospheric $\mathrm{O}_{2}^{+}$whereas in the closed magnetic field lines region, the $\mathrm{H}_{2} \mathrm{O}$ loss rate is lower by almost one order of magnitude and is mainly due to charge exchange between ionospheric $\mathrm{O}^{+}$and $\mathrm{H}_{2} \mathrm{O}$. The exospheric $\mathrm{O}_{2}$ net loss rate in the polar caps is likely due to electron impact ionization.

As at Ganymede, also at Europa, the dominant exospheric species is $\mathrm{O}_{2}$ due to its non-sticking property: in lack of sufficient energy to overcome Europa's gravity (Smyth \& Marconi 2006) it bounces multiply to the icy surface until it gets lost through ionization, charge-exchange or dissociation processes. As a result, a thin and dense exospheric envelope (with a thickness of some hundreds of $\mathrm{km}$ ), consisting mainly of thermal $\mathrm{O}_{2}$ molecules, accumulates around the moon (Plainaki et al. 2012, 2013). Plainaki et al. (2010a) estimated that the $\mathrm{O}_{2}$ mean free path in Europa's atmosphere ranges from $13 \mathrm{~km}$ to $78 \mathrm{~km}$. The scale-height estimations vary from $17 \mathrm{~km}$ to $26 \mathrm{~km}$ (Ip 1996; Plainaki et al. 2010a). Therefore, Europa's $\mathrm{O}_{2}$ tenuous atmosphere can be considered as a transitional case between a collisional and a collisionless (exosphere) environment. There are some uncertainties in the estimated column density of the molecular oxygen exosphere of Europa (ranging between $\sim 10^{14}$ and $10^{15} \mathrm{~cm}^{-2}$ ) because the Jovian magnetospheric electrons responsible for the observed UV auroral emissions can be partially diverted and cooled through interactions with the near-surface exosphere (Saur et al. 1998; Schilling et al. 2007). Indeed, such space weather phenomena evidenced in the magnetospheric electron spectrum and spatial distribution could be the determining factors for the observed auroral emissions studied several times in the past (see McGrath et al. 2004; Saur et al. 2011, 2015; Roth et al. 2014a, 2014b, 2016). Moreover, the exosphere generation and loss depend on the actual energetic particle and plasma distributions, respectively, around the moon. In Figure 17, it is shown that the density profile of the main exospheric species $\mathrm{O}_{2}$ at the Sun-subsolar point direction is subject to variations due to the varying impinging plasma and energetic particle distributions (note that the configurations between Europa, Jupiter and the Sun in Fig. 17 correspond to different orbital phases - see Plainaki et al. 2013 for details).

Recently, Bagenal et al. (2015) reviewed remote and in-situ observations of plasma properties at Europa's orbit, between Io's dense UV-emitting plasma torus and Jupiter's dynamic plasma sheet. These authors evaluated variations and uncertainties in plasma properties in space and in time providing a basis for further study of the plasma interaction with Europa. Lucchetti et al. (2016) used the state-of-the-art plasma model by Bagenal et al. (2015), based on original unexploited Galileo data, to calculate the loss rates of Europa's tenuous atmosphere due to different plasma-neutral processes. 

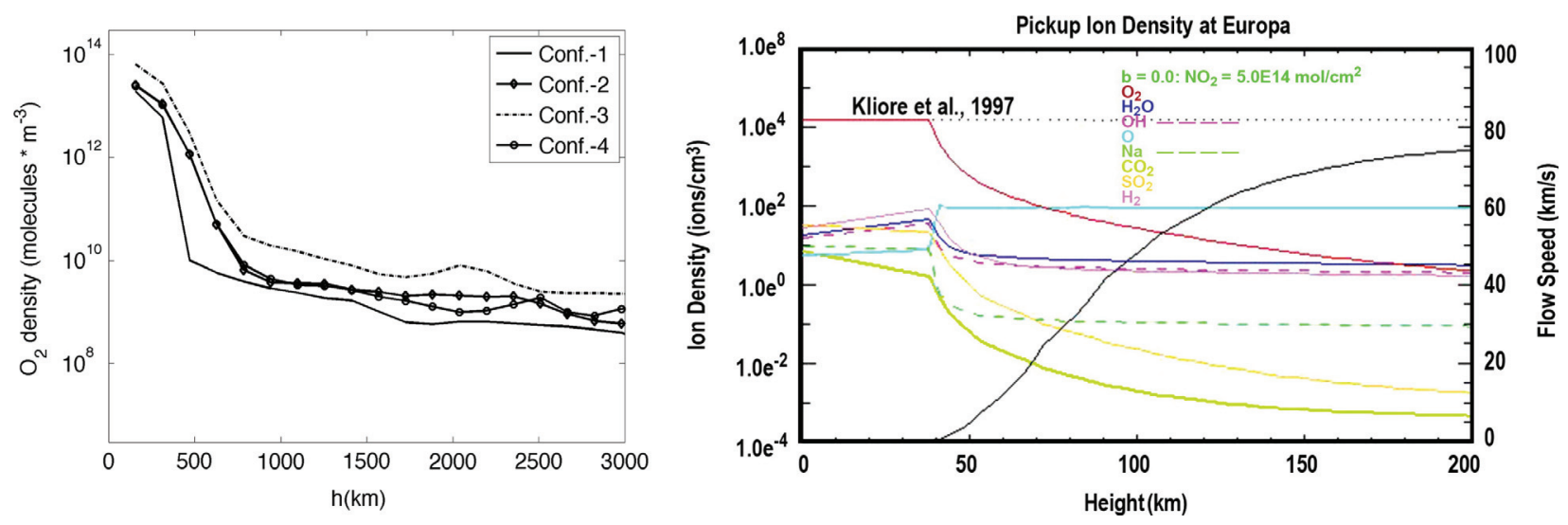

Fig. 17. (a): Density profile of the $\mathrm{O}_{2}$ exospheric species on Europa, in the Sun-subsolar point direction, as estimated with the EGEON model (Plainaki et al. 2012, 2013), accounting for the release-yield revisions (see Milillo et al. 2015; Plainaki et al. 2015). Different configurations correspond to different orbital phases hence to different orientations between plasma impact direction and illumination. (b) Ion density profiles at Europa. The dominant component, $\mathrm{O}_{2}^{+}$, the $\mathrm{H}_{2}{ }^{+}$population, water group ions (e.g. $\mathrm{O}^{+}, \mathrm{OH}^{+}$and $\mathrm{H}_{2} \mathrm{O}^{+}$) and the $\mathrm{Na}^{+}, \mathrm{SO}_{2}{ }^{+}$and $\mathrm{CO}_{2}{ }^{+}$trace species are considered. The flow speed (black line) of the plasma as it is mass loaded and eventually stops below the altitude of $\sim 40 \mathrm{~km}$ (ionopause), is also shown (right ordinate axis). From Sittler et al. (2013).

Due to the high variability of the plasma properties in the vicinity of Europa, the investigation was performed for three sample plasma environment cases identified by Bagenal et al. as hot/low density, cold/high density and an intermediate case. Therefore, the estimates by Lucchetti et al. (2016) referred indeed to the effect of the plasma environment variability in the actual loss of the moon's exosphere hence to the effects of space weather in the moon's neutral environment. Using the same plasma model, Dols et al. (2016) modelled the interaction of the Jovian magnetospheric plasma with the atmosphere of Europa, using a multi-species chemistry model, where the atmospheric distributions of $\mathrm{H}_{2}$ and $\mathrm{O}_{2}$ are prescribed. They estimated changes in the plasma composition resulting from this interaction as well as the reaction rates integrated over the simulation domain for several upstream plasma conditions (ion density, ion temperature and flow velocity). Both works by Lucchetti et al. (2016) and Dols et al. (2016) are relevant to future missions to Europa and to space weather in the moon's vicinity.

At Callisto the main component of the moon's surrounding neutral environment is believed to be $\mathrm{CO}_{2}$. Moreover, a recent study based on the analysis of HST data by Cunningham et al. (2015) provided evidence that Callisto has a significant atmosphere, which is probably denser than that of Europa or Ganymede and thus probably exceeded in mass among Solar System satellites only by the atmospheres of Io, Triton and Titan. The $\mathrm{O}_{2}$ atmosphere was found to be fully collisional even on the illuminated leading side of the moon, capable of supporting winds and other dynamical phenomena, protecting the surface from sputtering by magnetospheric particles. If this is the case, then contrary to Europa and Ganymede, at Callisto space weather is expected to depend mainly on the solar photon precipitating flux and its interactions with the atmosphere. An extended study of Callisto's neutral environment is also found in Vorburger et al. (2015).

In view of future missions to Jupiter, detailed and accurate knowledge of the conditions near the icy moons, in terms of atmosphere/exosphere density, scale height, ionosphere and plasma environment, is necessary. Moreover, the possible existence of water vapour at Europa, as suggested by Roth et al. (2014a), superimposed to the nominal exosphere, may lead to interactions between vapour and radiation and
UV environment. The neutral environment however tenuous is still an obstacle for the incoming magnetospheric ions and the ionospheric conductivity can divert the magnetospheric plasma flow around Europa (Saur et al. 1998). In order to be able to predict with accuracy the space weather conditions at an icy moon, one needs to determine the interactions and their variability based on both modelling and observation analysis. Some significant effort in this direction has already been made. Saur et al. (1998) developed a 2D plasma model to study the interaction of the Jovian magnetosphere with Europa's neutral environment and to identify sources and sinks that maintain the neutral $\mathrm{O}_{2}$ atmosphere. The 3D non-collisional Monte Carlo EGEON model by Plainaki et al. $(2012,2013)$ described the main exospheric components that are directly generated by ion sputtering and radiolysis. These authors also discussed the spatial variability of the exospheric environment expected at an icy moon and identified its relation to the direction of the magnetospheric ion fluxes impacting the surface (Plainaki et al. 2010a, 2012). In the context of planetary space weather, in particular, the variability along Europa's orbit of the $\mathrm{O}_{2}$ escape rate was studied in detail in Plainaki et al. (2013). It was also shown that the $\mathrm{O}_{2}$ exospheric densities at high altitudes are higher on the sunlit hemisphere, thus having a periodic modulation during the moon's orbit around Jupiter. Milillo et al. (2015) provided an analytical 3D model for Europa's $\mathrm{O}_{2}$ density, able to describe the molecular oxygen exosphere by reproducing the two-component profiles and the asymmetries due to different configurations among Europa, Jupiter and the Sun. The model by Milillo et al. (2015) was obtained by a non-linear fit procedure of the EGEON Monte Carlo model (Plainaki et al. 2012, 2013) to a Chamberlain density profile (Chamberlain 1963), simplified by Rairden et al. (1986). In view of space weather studies, this model is able to describe various exosphere properties through specific variable parameters thus allowing a detailed investigation of the exospheric characteristics. The $\mathrm{H}_{2}$ escape ratio is significantly higher and the hydrogen gas easily escapes from Europa's gravitational field (Plainaki et al. 2012). Smyth \& Marconi (2006) developed a 2D axisymmetric kinetic model considering ion-neutral collisions and assuming that the source rates for the various species $\left(\mathrm{H}_{2} \mathrm{O}, \mathrm{O}_{2}, \mathrm{H}_{2}\right.$ etc. $)$ were determined by partitioning the $\mathrm{O}_{2}$ source rate derived by the UV 
brightness of the O emissions reported by Hall et al. (1995). Shematovich et al. (2005) developed a 1-D collisional Monte Carlo model of Europa's atmosphere in which the sublimation and sputtering sources of $\mathrm{H}_{2} \mathrm{O}$ molecules and their molecular fragments are accounted for as well as the radiolytically produced $\mathrm{O}_{2}$. Similar efforts considering the generation and spatial distribution of the exospheres of Ganymede and Callisto, as a result of the plasma interactions with both surface and tenuous atmospheres, have been performed (see for example works by Marconi 2007; Turc et al. 2014; Plainaki et al. 2015 for Ganymede and Vorburger et al. 2015 for Callisto). Such investigations are of significant importance in the context of observations planning during future missions to the Jupiter's system (see also Sect. 3.2.1).

\subsection{Space weather in the inner Solar System}

Among the inner Solar System planets, Mercury is the one most subject to space weather since it is tightly linked to the Sun's activity. In fact, the proximity of Mercury to the Sun makes this planet and its environment a particularly interesting target to study extreme environmental conditions leading to unique Sun-planet coupling effects and consequent planet evolution. In the frame of space weather, Mars and Venus seem quite comparable for they both have a very similar atmospheric composition and no intrinsic magnetic fields. However, they exhibit very important differences. The distance from the Sun is one of the main factors, but not necessarily the key one. Venus, orbiting close to its parent-star, has a denser atmosphere than Mars. In Section 2.2.1 we describe the Mars and Venus atmospheres, discuss the magnetic fields - or rather their absence at the present time, and the potential implication of the solar activity and galactic cosmic rays. In Section 2.2.2 we discuss the extreme Mercury's environment where magnetosphere-IMF coupling determining the plasma-surface interactions becomes the dominant agent for space weather.

\subsubsection{Space weather at Mars and Venus}

Due to the planet's proximity to the Sun, the solar energy input at Venus has been too high to allow for long-term presence of oceans, leading to evaporation and to a massive accumulation of water vapour and $\mathrm{CO}_{2}$ in the atmosphere as well as to a runaway greenhouse effect. Water has been prone to massive loss in space, as suggested by the very high $\mathrm{D} / \mathrm{H}$ ratio (Donahue et al. 1997; Fedorova et al. 2008), enhanced by the direct erosion of the atmosphere by the solar wind; this is a major space weather effect, in the absence of an intrinsic magnetic field. The scenario of water evolution on Mars is still debated, but loss in space seems to have played again a major role, with this scenario being favourable due to the planet's lower mass (with respect to Venus) and lack of a global magnetosphere.

Figure 18 shows typical profiles of the thermospheric and ionospheric densities at Venus (see also Gronoff et al. 2008). The Venusian atmosphere above $95-100 \mathrm{~km}$ is characterized by a dynamical regime, dominated by a solar-antisolar circulation (Brecht \& Bougher 2012). The transition region around the altitude of $\sim 100 \mathrm{~km}$ hosts a number of peculiar phenomena such as the variable ozone layer (Montmessin et al. 2011) and strong variations in air temperatures (Piccialli et al. 2015). Incidentally, we note that this region is subject to high-energy deposition rates due to incoming cosmic rays during strong SEP events, as discussed by Nordheim et al.

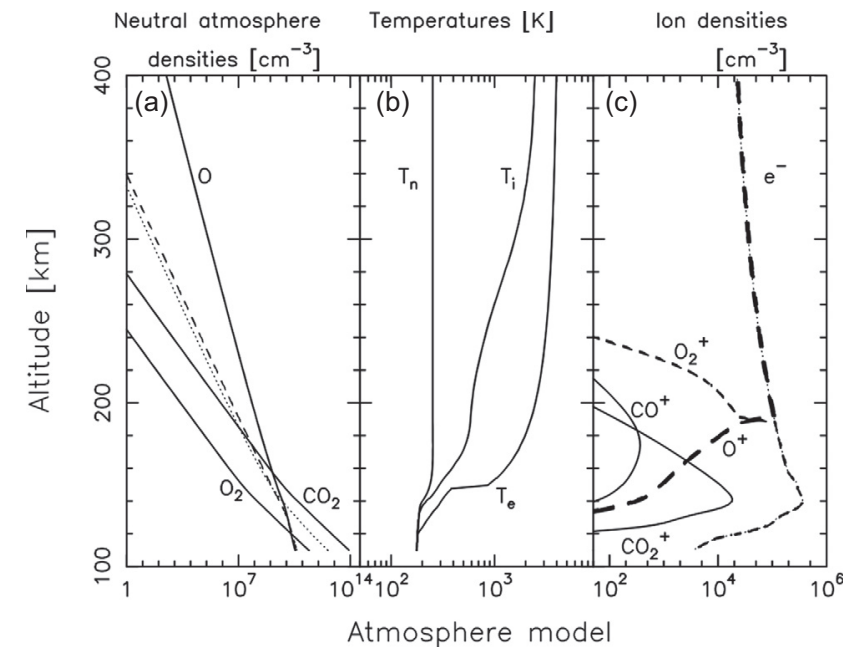

Fig. 18. Dayside upper atmosphere model for Venus. The dotted line (a) represents the $\mathrm{N}_{2}$ density and the dashed line the $\mathrm{CO}$ density. In (b) $T_{\mathrm{n}}$ is the neutral temperature, $T_{\mathrm{i}}$ the ion temperature and $T_{\mathrm{e}}$ the electron temperature. In (c) an ion density semi-empirical model is used. This model was computed for F10.7 = 80, latitude: $45^{\circ}$ at noon. From Gronoff et al. (2008).

(2015) and by Plainaki et al. (2016). Above the very rough level of $110 \mathrm{~km}$, dissociation of molecules by UV radiation becomes important. In particular, the dissociation of $\mathrm{CO}_{2}$ produces significant amounts of free oxygen at the dayside. These atoms experience recombination once they start drifting into the antisolar region, leading to the 1.27 and $1.58 \mu \mathrm{m}$ emissions by $\mathrm{O}_{2}\left(\mathrm{a}^{1} \Delta_{\mathrm{g}}-\mathrm{X}^{3} \Sigma_{\mathrm{g}}{ }^{-}\right)$, used as a powerful proxy for dynamics (Drossart et al. 2007). A similar scheme applies to the NO airglow UV emission, ultimately generated after the UV dissociation of $\mathrm{CO}_{2}$ and $\mathrm{N}_{2}$ at the dayside (Stiepen et al. 2013). Electron densities measured by radio-occultations (Pätzold et al. 2007) allow one to derive the overall structure of the ionosphere. A first layer (V1) is found around the altitude of $\sim 130 \mathrm{~km}$ and is caused by solar X-ray and impact ionization by photoelectrons. The main peak (V2) is located around the altitude of $\sim 140 \mathrm{~km}$ and is mostly attributed to the EUV ionization. A third, less defined layer (V3) is found around $\sim 180 \mathrm{~km}$ in altitude, and is likely induced by transport phenomena (Fox 2007; Peter et al. 2014 and references therein). Other aspects of the Venus atmosphere above the altitude of $\sim 100 \mathrm{~km}$ could not be investigated with the Venus Express payload, and the related information is based on older datasets. Considering the neutral species, $\mathrm{CO}_{2}$ dominates the composition of the atmosphere up to an altitude of $\sim 160 \mathrm{~km}$ on the dayside; above this altitude, the $\mathrm{CO}_{2}$-dissociation products ( $\mathrm{CO}$ firstly and then $\mathrm{O}$ ) surpass the $\mathrm{CO}_{2}$ content. Molecular nitrogen becomes lesser than the atomic one in the region between $170 \mathrm{~km}$ (nightside) and $200 \mathrm{~km}$ (dayside) (Von Zahn et al. 1983). Keeping in mind the very low content of water in the Venusian atmosphere, it is not surprising to measure very low contents of $\mathrm{H}_{2}$ and $\mathrm{H}$ (the latter always dominant) in the upper atmosphere (Keating et al. 1985). Considering the ionic species, there is evidence that $\mathrm{O}_{2}^{+}$is the main component below $180 \mathrm{~km}$ (Brace et al. 1983); above this altitude $\mathrm{O}^{+}$becomes dominant.

Our current knowledge of the upper Martian atmosphere has been summarized very recently in Fox (2015). Fundamental experimental data were derived from the observations of airglows by the Spectroscopy for Investigation of 
Characteristics of the Atmosphere of Mars (SPICAM) instrument on-board Mars Express: while NO is useful to constrain dynamics around the $100 \mathrm{~km}$ level (Gagné et al. 2013), CO and $\mathrm{CO}_{2}{ }^{+} \mathrm{UV}$ emissions allow the direct monitoring of the efficiency of ionization processes (Stiepen et al. 2015). Nonetheless, our knowledge on the complete profiles of several species still relies on data from the Viking entry probes (see Bougher et al. 2015a and references therein). The bulk composition of the neutral atmosphere remains very similar to the one observed at the surface up to the altitude of $\sim 140 \mathrm{~km}$, where the mixing ratio of oxygen begins to increase; finally, oxygen becomes the main neutral species above $\sim 200 \mathrm{~km}$. The ionosphere is dominated at most altitudes by $\mathrm{O}_{2}{ }^{+}$, with a density that reaches a maximum $\left(10^{5}\right.$ ions $\left./ \mathrm{cm}^{3}\right)$ around the altitude of $\sim 130 \mathrm{~km}$. Other important components are: the $\mathrm{CO}_{2}{ }^{+}$ species, peaking $\left(10^{4}\right.$ ions $\left./ \mathrm{cm}^{3}\right)$ at similar altitudes; the $\mathrm{NO}^{+}$ species, peaking $\left(10^{4}\right.$ ions $\left./ \mathrm{cm}^{3}\right)$ at the altitude of $\sim 100 \mathrm{~km}$; other important ionic species at lower altitudes. Electron densities have been extensively monitored by means of the Mars Express radio science experiment (Pätzold et al. 2009). The Martian thermosphere and ionosphere density profiles were recently modelled by Bougher et al. (2015b) and they are presented in Figure 19. Similarly to what was found on Venus, the main peak M2, located at the altitude of $\sim 130 \mathrm{~km}$ (Fox et al. 1996), is ascribed to UV ionization. Major new experimental constraints in this context are being collected from the Mars Atmosphere and Volatile Evolution (MAVEN) mission in orbit since September 2014. Since among the main goals of this mission are the characterization of atmospheric loss processes (with special attention to water) and the identification of their relation to the solar wind (Bougher et al. 2014), major scientific return related to space weather is expected in the next years.

The atmospheric escape is a strong evidence of planetary space weather effects at the terrestrial planets. Mars surface studies have shown that large quantities of liquid water were present in its early history (Parker 1989; Parker et al. 1993). If spread over the whole planet, this water ocean would have a depth of about $150 \mathrm{~m}$ (Carr \& Head 2003) and up to 1000 m (Baker et al. 2000; Clifford \& Parker 2001). In order to maintain liquid vapour under a $\mathrm{CO}_{2}$ atmosphere, a pressure of 1-3 bar is necessary, while today, the pressure is only about 4-9 mbar (Forget et al. 2009) above a quite dry soil. Whereas an in-depth discussion of the actual presence of water on Mars is beyond the scope of this article, we note that the abovementioned studies provide proof that the atmosphere of Mars escaped some time between the Noachian era (4.6-4.0 Gyrs) and the present. Several non-thermal processes related to this escape have been proposed in the literature. None of them is sufficient to explain the totality of the atmospheric escape, which necessitates taking into account a combination of all of them. The main mechanisms at work are (Chassefiere \& Leblanc 2004; Lundin et al. 2007; Lilensten et al. 2013): (a) photochemical escape, or dissociative recombination in which the ions produced by UV photoionization, electron impact and charge exchange with the solar wind protons, recombine generating escaping energetic neutrals; (b) pickup ion escape where the ions produced by photoionization are picked up by the solar wind; (c) atmospheric sputtering leading to exospheric neutrals undergoing collisions with energetic ions (mainly $\mathrm{O}^{+}$, initially formed from exospheric photoionization, being picked up and swept away by the solar wind magnetic lines, and finally re-impacting the exobase, provoking secondary escape); (d) hydrodynamic escape

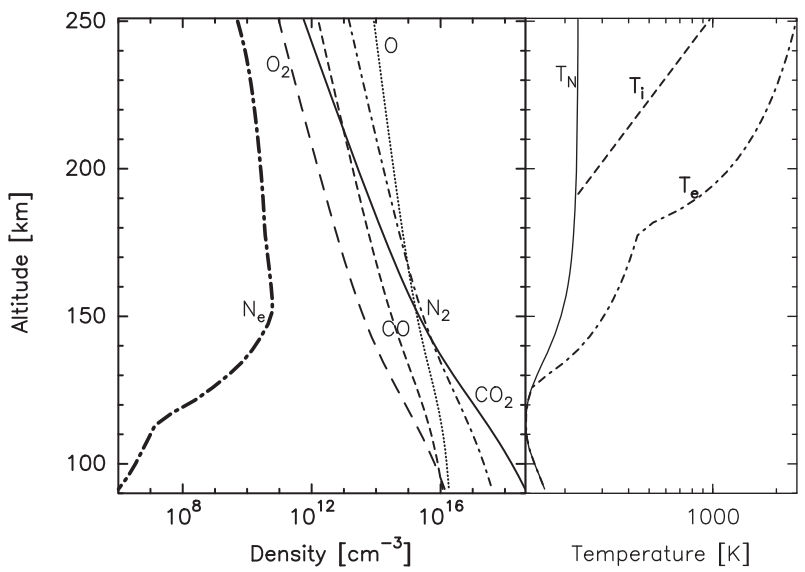

Fig. 19. Mars ionospheric and thermospheric profiles computed with 1D M-GITM (Bougher et al. 2015b) on January 15th, 2001, just before a flare at the location of MGS at $73^{\circ} \mathrm{E} 84^{\circ} \mathrm{N}$ with a solar zenith angle $1^{\circ}$.

(escaping light neutrals transferring momentum to heavier species), particularly during the Early Noachian era (Chassefiere \& Leblanc 2004). Therefore, the atmospheric escape at Mars has a strong connection to the solar activity (Luhmann et al. 1987; Lundin et al. 2008), since the solar EUV flux creates ions that can diffuse through the atmosphere and be picked up by the solar wind. In particular, the EUV solar flux has a strong influence on the atmospheric escape through two main mechanisms: (a) it ionizes and dissociates $\mathrm{CO}_{2}$ to form $\mathrm{CO}_{2}^{+}, \mathrm{CO}^{+}, \mathrm{O}^{+}$and some minor species such as $\mathrm{CO}^{++}$and $\mathrm{C}^{+}$; these daughter-ions are lighter than the neutral $\mathrm{CO}_{2}$ and diffuse upward, where a part of them gets picked up by the solar wind; (b) being unstable and highly energetic (i.e. with energy up to several $\mathrm{eVs}$ ), $\mathrm{CO}^{++}$dissociates in two fragments: $\mathrm{C}^{+}$and $\mathrm{O}^{+}$. The internal energy is released as kinetic energy, which is higher than the escape energy. These two mechanisms have been fully studied in Lilensten et al. (2013). Moreover, Chaufray et al. (2007) showed that the sputtering contribution to the total oxygen escape is smaller by one order of magnitude than the contribution due to the dissociative recombination. The two preceding mechanisms are the most easily tested against empirical data (Lundin et al. 2007). However, there are still a number of "nonthermal" electromagnetic acceleration processes that energize the plasma and lead to atmospheric escape. All together, these mechanisms are sufficient to explain the actual pressure observed at Mars. They all depend on the solar wind and on the solar flux, which varies in time, and may therefore be considered as space weather effects. Some other mechanisms are internal to Mars, such as volcanism (Brain et al. 1998; Gillmann et al. 2009; Grott et al. 2011) or release of surface material to the atmosphere due to meteoritic impacts. ASPERA on-board the ESA Mars Express probe (e.g. Lundin et al. 1990) showed that Mars is still experiencing atmospheric escape (Dubinin et al. 2008), with an escape flux ranging between $2 \times 10^{24}$ ions per sec and $3 \times 10^{25}$ ions per sec (Lundin et al. 1990; Lundin et al. 2008; Nilsson et al. 2011).

Observation by the MAVEN spacecraft of the upper Mars atmosphere during a CME showed an enhancement in the escape rate of ions to space during the event, suggesting that ion loss during solar events in early Mars history may have been a major contributor to the long-term evolution of the Mars atmosphere (Jakosky et al. 2015). 
The case of Venus is quite different. Due to its proximity to the Sun, one would expect a larger escape flux. However, this is not the case. Masunaga et al. (2014) used ASPERA 4 on-board ESA Venus Express to estimate a total $\mathrm{O}^{+}$escape rate of $(5.8 \pm 2.9) \times 10^{24} \mathrm{~s}^{-1}$, during perpendicular IMF, and $(4.9 \pm 2.2) \times 10^{24} \mathrm{~s}^{-1}$, during horizontal IMF; larger rates are expected during CMEs (Luhmann et al. 2007, 2008). Coates et al. (2015) suggested that the presence of photoelectrons in the tail, together with low-energy ions, is possible evidence for a polar wind style escape at Venus, along the draped magnetic field around the planet. Through two distinct examples, they showed the effect of this additional escape and estimated a loss rate of $2.2 \times 10^{23} \mathrm{~s}^{-1}$ with an error up to $50 \%$. The lower escape rates (with respect to their values on Mars) are mainly due to Venus' larger gravity. The escape velocity is $10.4 \mathrm{~km} / \mathrm{s}$ at Venus while it is only half of this value at Mars. While several mechanisms may give enough energy to the atoms to escape Mars, they are not efficient to accelerate them enough to escape Venus (Lilensten et al. 2013), except during periods of strong solar events (Luhmann et al. 2007, 2008). Therefore, depending on the planet, the atmospheric escape is the effect of the regular solar state (e.g. at Mars) or of its variability hence of the planetary space weather conditions in the planet's neighbourhood (e.g. not only at Venus, but also at the Earth).

The thermospheric glows at Mars and Venus are characterized by the importance of the CO Cameron and fourth positive bands below $270 \mathrm{~nm}$ (Fox \& Bougher 1991; Leblanc et al. 2006a, 2006b). Other important emissions are the ones of atomic oxygen, especially the red and green lines, and the one of $\mathrm{CO}_{2}^{+}$. In particular, it has recently been proposed that the Fox-Duffendack-Barker bands must be observable in Mars, especially in the blue part (Lilensten et al. 2015). Both Mars and Venus upper thermospheres are mainly composed of atomic oxygen. The main emissions of this atom are the red line, the green line and the 130.4 and $135.6 \mathrm{~nm}$ multiplet. The deactivation of the $\mathrm{O}\left({ }^{1} \mathrm{~S}\right)$ oxygen state creates the green line $\mathrm{O}\left({ }^{1} \mathrm{~S}\right) \rightarrow \mathrm{O}\left({ }^{1} \mathrm{D}\right)$ at $557.7 \mathrm{~nm}$ (94\% of the emission) and the $297.2 \mathrm{~nm}$ emission $\left(\mathrm{O}\left({ }^{1} \mathrm{~S}\right) \rightarrow \mathrm{O}\left({ }^{3} \mathrm{P}\right)\right)(6 \%)$. After a first detection in 1999 (Slanger et al. 2001, 2004), the green line has been recently observed at Venus after solar flares and CMEs (Gray et al. 2014). The red line $\left(\mathrm{O}\left({ }^{1} \mathrm{D}\right) \rightarrow \mathrm{O}\left({ }^{3} \mathrm{P}\right)\right)$ is actually a triplet at $630,636.3$ and $639.1 \mathrm{~nm}$ produced through: electron impact on $\mathrm{O}$; dissociative recombination of $\mathrm{O}_{2}^{+}$; photodissociation of $\mathrm{O}_{2}$; thermal electron impact on $\mathrm{O}$; cascading from $\mathrm{O}\left({ }^{1} \mathrm{~S}\right)$ state; dissociative recombination of $\mathrm{CO}^{+}$; quenching of the $\mathrm{O}\left({ }^{1} \mathrm{~S}\right)$ state that produces $\mathrm{O}\left({ }^{1} \mathrm{D}\right) ; \mathrm{CO}_{2}$ photodissociation; and chemical reactions of $\mathrm{O}_{2}$ with $\mathrm{N}\left({ }^{2} \mathrm{D}\right)$ and $\mathrm{N}^{+}$. The other main emissions of the atomic oxygen are the $844.6 \mathrm{~nm}$ line which corresponds to the $\mathrm{O}\left({ }^{3} \mathrm{P}\right)$ to $\mathrm{O}\left({ }^{3} \mathrm{~S}\right)$ transition and the triplet at $130.2,130.4$ and $130.6 \mathrm{~nm}$ due to the unstable $\mathrm{O}\left({ }^{3} \mathrm{~S}\right)$ state.

The aurorae at Mars have been discovered in 2005 during the Mars Express mission. Their detection by Bertaux et al. (2005) was made using the UV channel of the SPICAM instrument. These aurorae are located close to vertically aligned crustal magnetic fields and have been largely studied since then in an attempt to determine the exact process for their creation and their potential effect on the upper atmosphere of Mars (Leblanc et al. 2006a, 2006b; Brain et al. 2012). We note, however, that these aurorae have only been studied in the UV spectral range. On Venus, continuous and highly variable emissions at $130.4 \mathrm{~nm}$ have been observed at the nightside by the Pioneer Venus Orbiter ultraviolet spectrometer (Phillips et al. 1986) with intensities occasionally exceeding $100 \mathrm{R}$. These "auroral" emissions are attributed to the precipitation of suprathermal electrons associated with solar flares and/or interplanetary shocks. Recently, signatures of $\mathrm{CO}$ Cameron and $\mathrm{CO}_{2}^{+}$doublet ultraviolet auroral emissions have been detected with SPICAM (Gérard et al. 2015); they showed that the Mars aurora is a temporary and spatially localized phenomenon appearing near the open-closed magnetic field line boundary in cusp-like structures.

Atmospheric ionization by the impinging GCR is another effect of planetary space weather. Venus is constantly being bombarded by cosmic rays, high-energy charged particles of solar, galactic and extragalactic origin. Below the penetration depth of solar EUV and X-ray photons $(<100 \mathrm{~km})$, solar and galactic rays are the primary ionization source in the Venusian atmosphere (Borucki et al. 1982). At an orbital distance of 0.72 $\mathrm{AU}$, Venus is exposed to high particle fluxes from sporadic SEP events and CMEs. Due to the absence of a global magnetic field cosmic rays have unimpeded access to the Venusian atmosphere and due to a total shielding depth of $10^{5} \mathrm{~g} / \mathrm{cm}^{2}$ (the terrestrial value is $10^{3} \mathrm{~g} / \mathrm{cm}^{2}$ ) (Borucki et al. 1982), cosmic ray air showers develop extensively.

As cosmic rays represent the major ionization source in the Venusian middle and lower atmosphere (below $\sim 100 \mathrm{~km}$ ), cosmic ray ionization rates have a strong influence on fundamental atmospheric properties such as electrical conductivity, atmospheric chemistry and charging of cloud particles (Dubach et al. 1974; Borucki 1982; Michael et al. 2009; Aplin 2013; Nordheim et al. 2015). It has also been suggested that aerosols may form by direct condensation of gaseous sulphuric acid onto ions in the lower atmosphere, thus providing a possible link between cloud formation and cosmic ray ionization in the Venusian atmosphere (Aplin 2006, 2013). Atmospheric ionization due to the background flux of GCR particles peaks within the main cloud deck at $\sim 63 \mathrm{~km}$ and recent modelling by Nordheim et al. (2015) and Plainaki et al. (2016) has shown that transient SEP events may enhance ionization rates at this altitude by more than an order of magnitude and by up to five orders of magnitude at higher altitudes ( 80-100 km; Plainaki et al. 2016). Extreme SEP events may yield even larger enhancements in atmospheric ionization rates, and the impact of "Carrington-level" SEP events on the Venusian atmosphere was recently investigated by Dartnell et al. (2015). Thus, several important atmospheric properties may in turn be sensitive to variability in cosmic ray conditions at Venus both over long (solar cycle) and short (SEP events) timescales.

While the large atmospheric pressure at the surface and the high altitude of the Venusian cloud layer appears to exclude the possibility of cloud-to-ground lightning (Gurnett et al. 2001; Aplin 2006), several authors have suggested that lightning discharges above, between or within clouds may occur (Borucki 1982; Russell \& Scarf 1990; Gurnett et al. 2001). Positive detections of radio wave emissions due to Venusian lightning have been reported (Ksanfomaliti et al. 1979; Taylor et al. 1979; Russell et al. 2007). However, the lack of radio observations by the Cassini spacecraft during two close flybys and the lack of optical detections by Venus Express as well as groundbased observers leave the question of Venusian lightning somewhat unresolved (Yair et al. 2008; Yair 2012). While the exact mechanism for charging and for initiating lightning within the Venusian clouds is not currently known (Yair 2012) it is nonetheless likely that cosmic ray ionization has an important role in this process as it is believed to be the primary source of 
atmospheric ions at middle and low altitudes. Thus, it is plausible that changes to atmospheric ionization rates due to cosmic ray variability may affect the occurrence of electrical phenomena in the Venusian atmosphere (Nordheim et al. 2015) hence manifesting a relation between space weather and meteorological phenomena.

Large changes in atmospheric ionization induced by sporadic SEP events may potentially also affect radio wave propagation in the Venusian atmosphere. At Mars, the MARSIS radar instrument on-board Mars Express has experienced sporadic blackouts (Withers 2011) and recent modelling by Norman et al. (2014) suggestthat this may be due to enhanced ionization from SEP events. As SEP events may produce very large enhancements in the ion production rate in the Venusian upper and middle atmosphere, investigations of space weather effects at Venus are relevant to future missions to the planet, particularly those carrying lowfrequency radar instruments (Nordheim et al. 2015).

The Martian atmosphere, with its significantly lower total shielding depth $\left(\sim 16 \mathrm{~g} / \mathrm{cm}^{2}\right.$, according to Kminek \& Bada 2006 ) is not capable of significantly attenuating energetic GCR and SEP particles. Indeed, for GCR particles, the peak in secondary particle production typically occurs within the top metre of the Martian subsurface (Simonsen \& Nealy 1993; Molina-Cuberos 2001; Gurtner et al. 2006; Dartnell et al. 2007a, 2007b), while SEP particles are capable of affecting the shallow subsurface to depths of $\sim 10 \mathrm{~cm}$ (Dartnell et al. 2007b). While, contrary to Venus, Mars possesses significant local crustal magnetic fields (e.g. Acuña et al. 1999), it is nevertheless not significantly shielded from GCR and SEP particles (Dartnell et al. 2007a). At Mars, cosmic rays are therefore capable of strongly affecting conditions at the surface and within the near subsurface. This has strong implications for astrobiology and planetary habitability as any putative life forms near the surface would be exposed to large radiation doses capable of sterilizing the top $\sim 20 \mathrm{~cm}$ of the Martian subsurface (Simonsen \& Nealy 1993; Mileikowsky et al. 2000; Pavlov et al. 2002; Dartnell 2011; Dartnell et al. 2007a, 2007b, 2010). Additionally, cosmic ray irradiation may destroy complex organics at the surface and within the near subsurface (Kminek \& Bada 2006; Pavlov et al. 2012), with implications both for surface chemistry and for the search for ancient biomarkers on Mars. The large cosmic ray-induced radiation dose at the surface may also prove problematic for future human exploration of Mars (e.g. Saganti et al. 2004).

Cosmic ray ionization in the Martian atmosphere has been studied extensively using computational models (Whitten et al. 1971; Molina-Cuberos 2001; Molina-Cuberos et al. 2001; Norman et al. 2014; Gronoff et al. 2015), with the general result that the atmospheric ionization profile due to GCR is relatively flat, with monotonically increasing ionization rates with decreasing altitude and increasing atmospheric density. Recent in-situ measurements at the Martian surface by the RAD instrument (Hassler et al. 2012) on board the Mars Science Laboratory rover agree relatively well with the quiet-time GCR predictions made by these computational modelling efforts (Hassler et al. 2014; Kim et al. 2014; Gronoff et al. 2015).

Unlike the quiet-time background GCR flux, SEP events have been shown to produce large altitude-dependent enhancements in atmospheric ionization, with the altitude of the ionization peak depending strongly on the shape of the SEP primary spectrum (Sheel et al. 2012; Norman et al. 2014; Gronoff et al. 2015). In-situ measurements by RAD have revealed that the relatively moderate SEP event of 11 April 2013 produced a 30\% enhancement over background dose rates at the Martian surface (Hassler et al. 2014). Recent computational studies have shown that strong SEP events may produce ionization and dose rate enhancements of more than four orders of magnitude both at altitude in the Martian atmosphere and at the surface (Norman et al. 2014; Gronoff et al. 2015).

\subsubsection{Space weather at Mercury}

Mercury possesses a weak intrinsic global magnetic field (discovered by Mariner 10; Vilas et al. 1988) that supports a small and dynamic magnetosphere, though hardly able to protect the planet from the Sun's action. For this reason, Mercury's environment is a complex and tightly coupled system where the magnetosphere, exosphere (see Fig. 20) and surface are linked by interaction processes that facilitate exchange of both material and energy (Killen \& Ip 1999; Killen et al. 2001; Milillo et al. 2010). Investigations regarding the coupling of Mercury's magnetosphere with the IMF, as well as the planet's interaction with the Sun (in terms of both electromagnetic and energetic particle radiation) and with interplanetary dust, can provide important clues for the planet evolution from the Solar System formation to now (Orsini et al. 2014). Basically, the resulting effects and types of interactions along the planet's orbit, at such a close distance from the Sun, are among the major Solar System key-questions to be answered in the future.

The Parker spiral forms an angle of about $20^{\circ}$ with the solar wind radial direction, less than half of its value at the Earth's orbit $\left(\sim 45^{\circ}\right)$; this implies a change of the relative ratio of the IMF components with respect to the near-Earth conditions and an increase of the weight of the IMF $B x$ component (Massetti et al. 2003). The solar wind is much more intense at Mercury's orbit than at any other planet of the Solar System (Burlaga 2001). Although the solar wind velocity remains relatively constant throughout the heliosphere, its density at Mercury's orbit is 5-10 times larger than its typical values at the Earth. Additionally, the strength of the IMF is higher, having, on average, a value of $30 \mathrm{nT}$ (Raines et al. 2014). Therefore, solar wind Alfven speed is high, the Mach number is low and the reconnection rate with Mercury's magnetic field is high (Slavin \& Holzer 1979). We note that Mercury's intrinsic magnetic field has an intensity equal to $2 \times 10^{-7} \mathrm{~T}$ at the equator.

The recent magnetic field measurements of the NASA MErcury Surface, Space ENvironment, GEochemistry, and Ranging (MESSENGER) mission, operated around Mercury from 2008 to April 2015 (Solomon et al. 2007), indicate that Mercury's magnetic dipole moment is offset northward from the planet's centre by $0.2 R_{\mathrm{M}}$ (where $R_{\mathrm{M}}$ is Mercury's radius, equal to $2440 \mathrm{~km}$ ) (Alexeev et al. 2010; Anderson et al. 2011a). The relative dimension of Mercury with respect to the dimension of its own magnetosphere is much larger than in the Earth's case (see also Fig. 1). At Mercury, the average subsolar magnetopause standoff distance is $\sim 1.45 R_{\mathrm{M}}$ (Winslow et al. 2013) whereas at the Earth it is $\sim 10 R_{\mathrm{E}}$ (Fairfield 1971). Upstream of the magnetosphere, Mercury's bow shock is located at an average distance of $1.96 R_{\mathrm{M}}$ away from the planet (Winslow et al. 2013). We now know that 


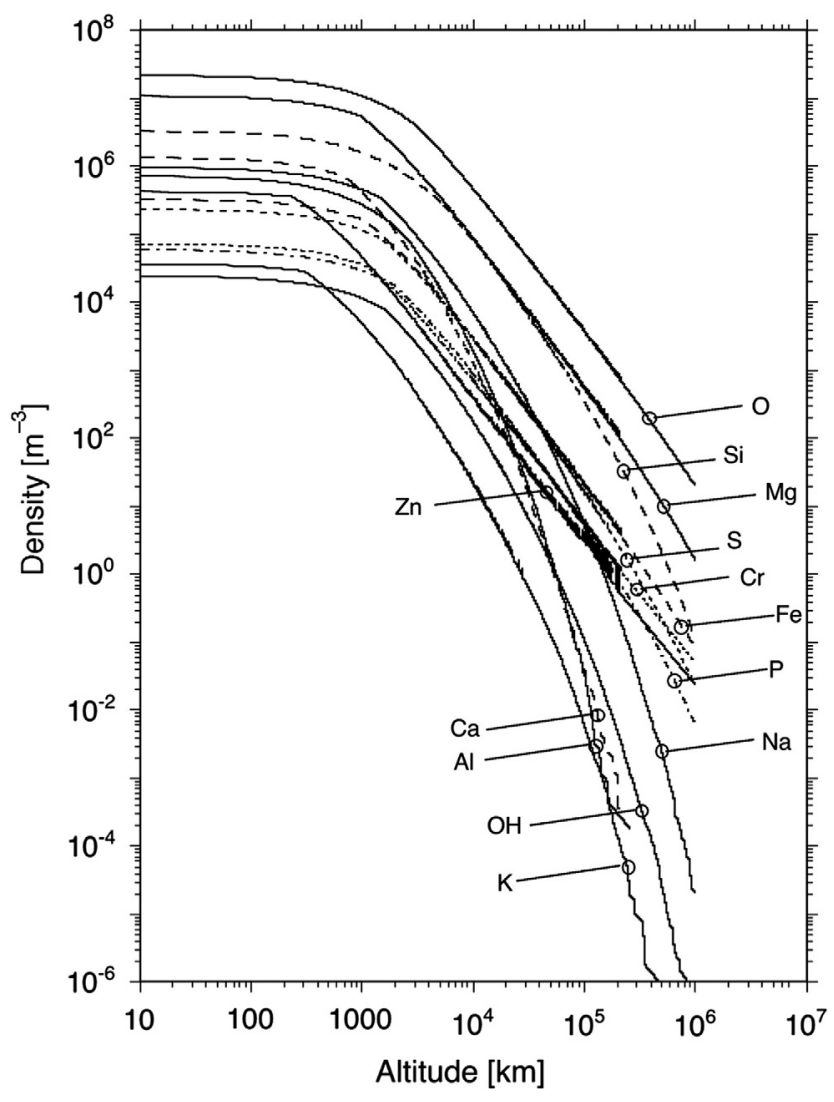

Fig. 20. Modelled density profiles of different species in the exosphere of Mercury, released from the surface through sputtering. From Wurz et al. (2010).

Mercury's magnetosphere is highly dynamic (e.g. Slavin et al. 2009, 2010; DiBraccio et al. 2013), so it cannot be considered as a stable structure where plasma distributes according to well-characterized populations, like in the Earth's magnetosphere. On the contrary, fast (few seconds) events like Flux Transfer Events (FTEs; Slavin et al. 2012b), depolarizations (Sundberg et al. 2012) and plasmoids (Slavin et al. 2012a) are observed. Given Mercury's iron-rich electrically conducting core (Smith et al. 2012), Hood \& Schubert (1979) and Suess \& Goldstein (1979) predicted that induction effects at Mercury would cause the subsolar magnetopause to remain at or above the distance of $1.2 R_{\mathrm{M}}$. In fact, changes in upstream solar wind pressure will create changes in the magnetic field normal to the planetary surface. According to Faraday's law, these time-dependent changes will generate currents in the conducting core, which will serve to oppose this change in magnetic field and temporarily increase Mercury's magnetic moment (Hood \& Schubert 1979; Suess \& Goldstein 1979; Glassmeier et al. 2007). Slavin et al. (2014) analysed three extreme solar wind dynamic pressure events using MESSENGER magnetic field and plasma measurements, confirming that the magnetopause standoff distance will only be compressed below $\sim 1.2 R_{\mathrm{M}}$ for solar wind pressures larger than $\sim 60 \mathrm{nT}$.

To characterize how the coupled system dynamically responds to the external forcing, Jia et al. (2015) developed a global resistive MHD model that also explicitly includes the planetary interior with layers of different conductivities by using time-dependent solar wind conditions. They demonstrated that the induction effect plays an important role in determining the global magnetospheric structure. The solar wind entering the magnetosphere of Mercury through its large cusps can circulate or alternatively reach the surface. The solar wind impacting region, in a first approximation, corresponds to the footprint of the open field lines; the position and dimension of this footprint depend on solar wind conditions (Kallio \& Janhunen 2003, 2004; Massetti et al. 2003). Evidences of proton fluxes precipitating toward the surface have been registered recently by MESSENGER/FIPS data below the Northern cusps (Zurbuchen et al. 2011).

Together with the protons and $\mathrm{He}^{2+}$ of solar wind origin, FIPS and Mercury Atmospheric and Surface Composition Spectrometer (MASCS) detected ions of planetary origin (McClintock \& Lankton 2007; Zurbuchen et al. 2008; Vervack et al. 2010) both inside and outside of the magnetosphere. The distributions of the two most abundant species, i.e. the $\mathrm{Na}^{+}$and $\mathrm{O}^{+}$-group ions, show an abundant enhancement in the region of Mercury's northern magnetospheric cusp. $\mathrm{Na}^{+}$-group and $\mathrm{O}^{+}$group ions are also very abundant in the nightside near-equatorial region, and often near high-latitude, dayside crossings of the magnetopause (Zurbuchen et al. 2011; Raines et al. 2013). Raines et al. (2014) focused on the $\mathrm{Na}^{+}$-group energy, flow direction and spatial distribution in Mercury's Northern cusp region (Fig. 21). These authors argued that the high energies $\left(\sim 2.7 \mathrm{keV}\right.$ on average) of the $\mathrm{Na}^{+}$-group ions in Mercury's cusp demonstrate that these populations have not been produced locally in the cusp, but they must have been previously accelerated. They also found a regular occurrence of keV-energy $\mathrm{Na}^{+}$-group ions flowing northward in the dayside magnetosphere. From these measurements, the authors hypothesized that neutral $\mathrm{Na}$ atoms were ionized in the vicinity of the subsolar magnetopause and accelerated into the cusp through reconnection.

A significant electron population has been observed at Mercury. Electrons within the 200-680 eV energy range were already detected by Mariner 10 and interpreted as coming from a hot plasma sheet (Ogilvie \& Desch 1977). Furthermore, bursts of low- and moderate energy electrons (tens to hundreds of keVs, Ho et al. 2012) have been often recorded by the MESSENGER Energetic Particle Spectrometer (EPS) and X-Ray Spectrometer (XRS). A systematic monitoring permitted to map the burst occurrence. The largest burst events were either at high (northern) latitudes or near local midnight, and similar in particle intensity, spectra and pitch angle. They are interpreted as the result of acceleration by the same mechanism. Other electron observed events require further studies for complete explanations: lower-energy events were also seen near the equator, but they were mostly absent in both the dawn and dusk local time sectors. Another group of events occurred upstream of Mercury's bow shock, similar to upstream events found at Earth during which particles are accelerated at the bow shock and subsequently travel upstream into the solar wind.

The SEPs (electrons and $\mathrm{H}^{+}, \mathrm{He}^{++}, \mathrm{C}^{6+}, \mathrm{O}^{7+}$ and $\mathrm{Fe}^{12+}$, with energies in the $\mathrm{MeV}-\mathrm{GeV}$ range) ejected from the base of the solar corona and above, in association with flares or CMEs (Kahler et al. 1984), may encounter Mercury along their propagation path, fulfilling the planet's environment with a significant flux of energetic particles. Leblanc et al. (2003), through simulations of SEP trajectories inside Mercury's magnetosphere, showed that significant SEP fluxes can reach Mercury's surface not only into the cusps footprint, as in the case of solar wind, but also at the planet's equator, elongated toward dawn. 
(a)

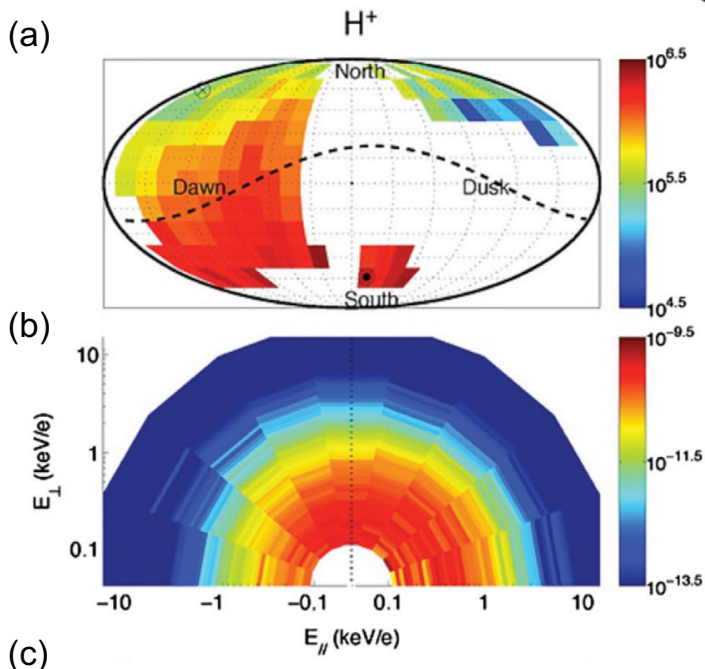

(c)

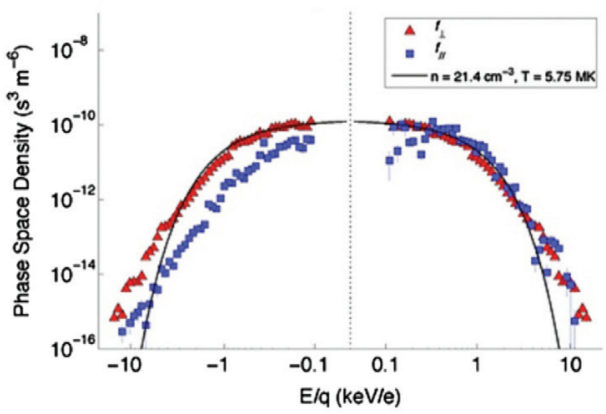

Cusp

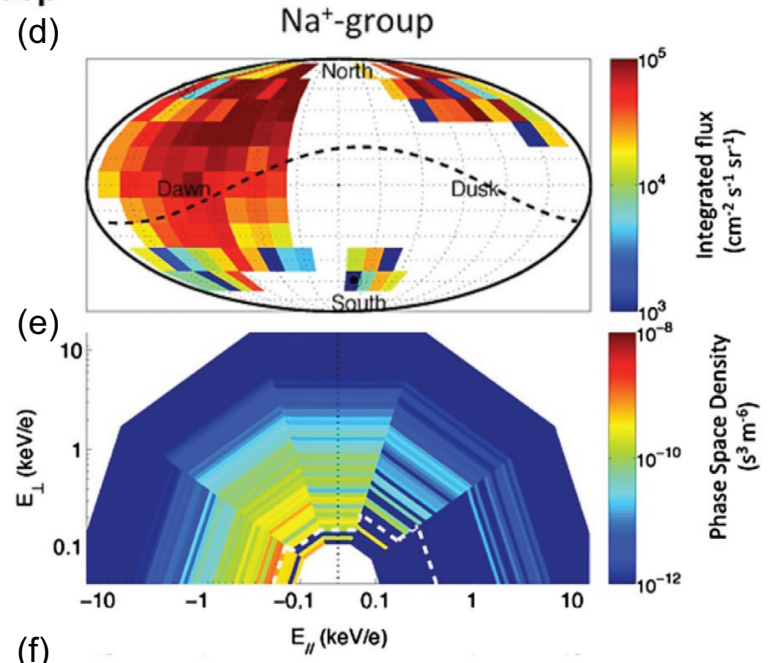

(f)

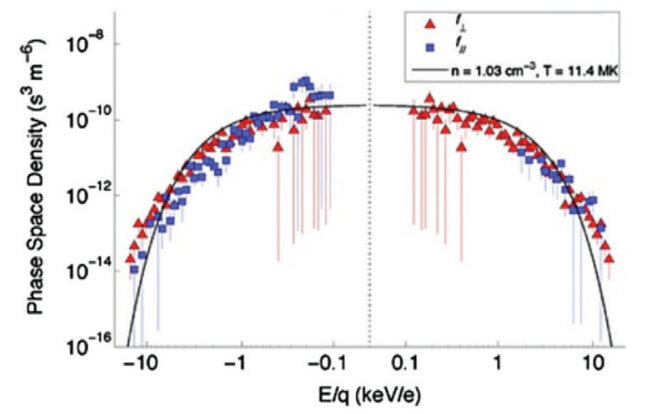

Fig. 21. Kinetic properties of protons and $\mathrm{Na}^{+}$-group ions within the cusp, accumulated over 77 cusp crossings. Top panels (a, d) show flow direction histograms for protons and $\mathrm{Na}^{+}$-group ions. The middle panels $(\mathrm{b}, \mathrm{e})$ are energy-resolved pitch angle distributions, which show the flow direction and energy of ions relative to the magnetic field in $20^{\circ}$ (protons) and $36^{\circ}\left(\mathrm{Na}^{+}\right.$-group) bins. Slices through these distributions in the parallel, anti-parallel and perpendicular directions are shown in the bottom panels (c, f). These figures show protons which are flowing down toward the surface, as well as loss cone of $>40^{\circ}$ in width. Low-energy $(100-300 \mathrm{eV}) \mathrm{Na}^{+}$-group ions appear to be upwelling from the surface, while those at energies up to $10 \mathrm{keV}$ have large perpendicular energy components. Reproduced from Raines et al. (2014).

Extensive penetration of pick-up ions into the magnetosphere was found in the post-noon and dusk local time sectors at the time of MESSENGER's first flyby during northward IMF conditions (Sarantos et al. 2009). These pick-up photoions from magnetosheath may be an important source of hot planetary ions within the magnetosphere (through the Kelvin-Helmholtz instability).

The effects of the planetary space weather are also strongly evident in the exosphere characteristics. Mercury's exosphere comprises many species, reflecting the surface composition according to the release process efficiencies (Wurz \& Lammer 2003). The fluxes released from Mercury's surface through different mechanisms, and thus the associated exospheric densities, were estimated by Wurz et al. (2010). In Figure 20, we present the modelled density profiles of the species released through sputtering (Wurz et al. 2010). In fact, the particles are released from the surface mainly after the action of solar UV and/or IR radiation as well as solar wind precipitation. Depending on both Sun's activity and distance along the eccentric orbit of Mercury, the exosphere varies both in intensity and morphology (e.g. Killen \& Ip 1999; Mangano et al. 2015); in addition, the radiation pressure produces a $\mathrm{Na}$ tail with an elongation variation from a few $R_{\mathrm{M}}$ up to more than a thousand $R_{\mathrm{M}}$ (Potter et al. 2002; Schmidt et al. 2010). In Figure $22 \mathrm{a}$, some examples of $\mathrm{Na}$ variability in the disk are shown. The solar wind and planetary ions interact with the surface to produce ion sputtering, backscattering and internal structure alteration via chemical sputtering and/or enhanced diffusion (Mura et al. 2009; Sarantos et al. 2009). Furthermore, micrometeoroid impact vaporization plays a role in the exosphere generation too, which may overlap the effects linked to the Sun (Leblanc \& Johnson 2003; Killen \& Hahn 2015). In this complicated picture, the neutral environment observations should be interpreted by evaluating the most important mechanisms depending on species, True Anomaly Angle (TAA), Local Time (LT) and longitudinal and surface features. The debate on the most important surface release process acting on Mercury remains open within the scientific community: it is a key-point for constraining the planet's long- and short-term evolutionary scenario.

The mostly observed neutral species at Mercury is Na, thanks to its simple optical emission doublet, directly identified from ground-based telescopes, and to its high density in the planet's environment. The $\mathrm{Na}$ emission exhibits often a typical morphology with two peaks at mid-latitudes in the dayside, inside regions possibly linked to the magnetic cusp footprints on the surface (see Fig. 22b). Nevertheless, despite the many exospheric observations from the Earth and from space (with MESSENGER), a comprehensive explanation of the totality of the $\mathrm{Na}$ measurements is not yet existent in the literature. On one side, as previously stated, the configurations of the $\mathrm{Na}$ exosphere (similarly to the $\mathrm{K}$ exosphere), derived from 
(a)

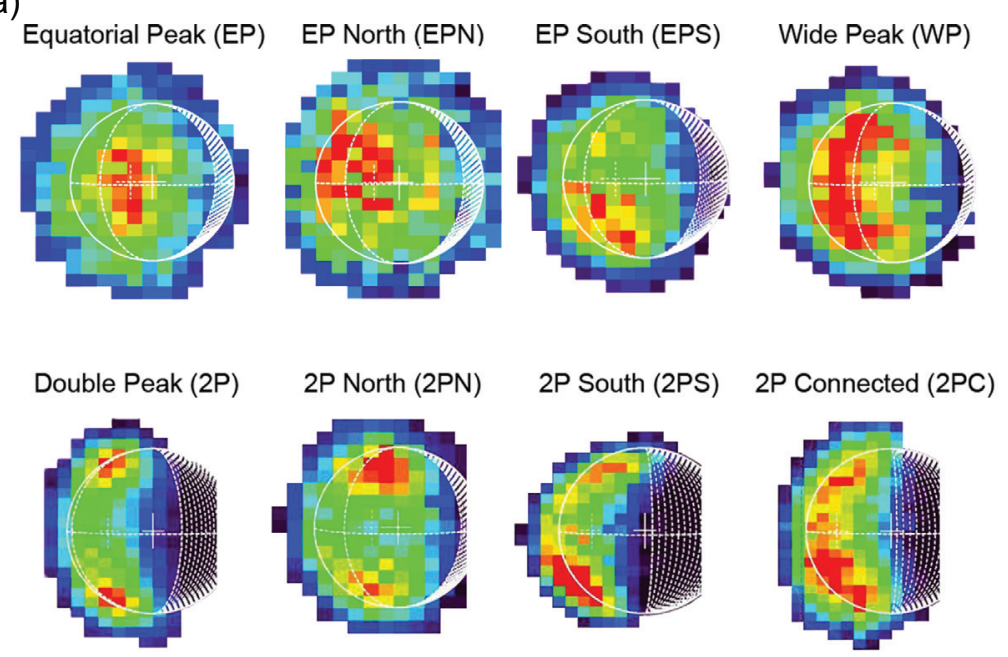

(b)

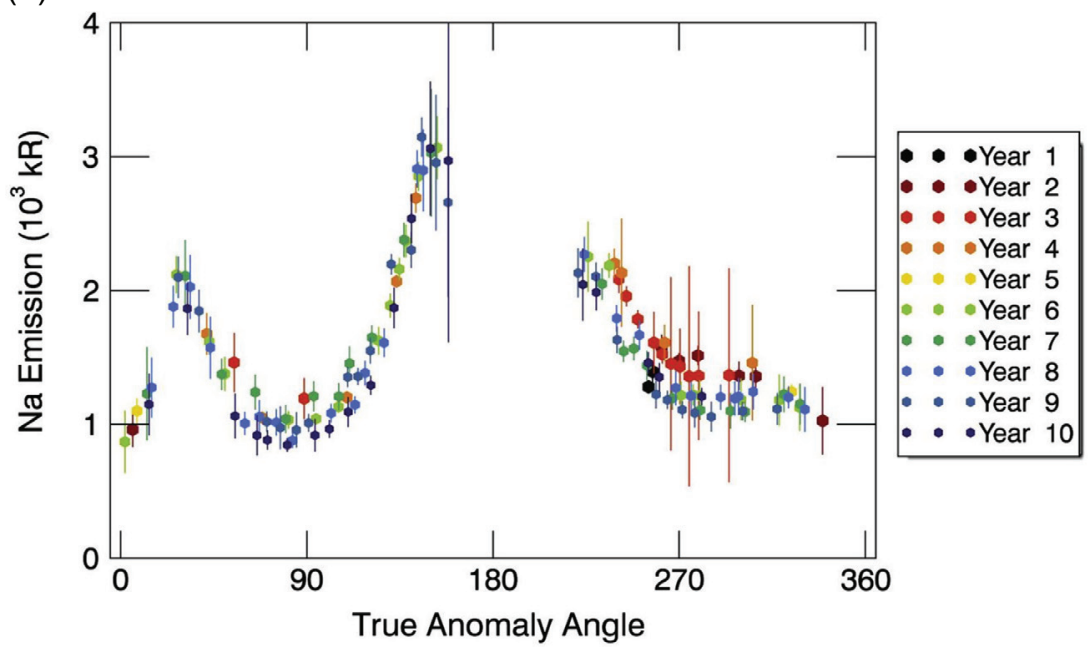

Fig. 22. (a) Examples of different Na-emission patterns identified in the Hermean exosphere through ground-based observation with the THEMIS solar telescope. From Mangano et al. (2015). (b) Surface sodium density estimated from observations near the subsolar point, as a function of the true anomaly angle. From Cassidy et al. (2015).

Earth ground-based observations, suggest a strict relation of the exosphere generation with the plasma precipitation regions, varying in timescale of hours. The statistical correlation with the $z$-component of the IMF with the double peak configuration (Mangano et al. 2015) indeed seems to favour a major role of plasma impact in the cusps projection onto the surface. On the other side, the intensity of such an exospheric signal is hardly consistent with a simple ion sputtering release (e.g. Leblanc et al. 2008), thus a more complex scenario (e.g. combination of several processes) should be invoked to account for this discrepancy (Mura et al. 2009). Indeed, such scenarios have already been proposed in the past. In particular, it has been suggested that the ion precipitation not only produces direct sputtering, but also enhances the release due to photon stimulated desorption, either through the breaking of the $\mathrm{Na}$ atomic bond to the surface (chemical sputtering, see Mura et al. 2009) or through the enhancing of the $\mathrm{Na}$ diffusion inside the regolith grains (Sarantos et al. 2009). Furthermore, the MESSENGER UV observations do not confirm this variability as well as the double peak configuration. On the contrary, the MESSENGER observations indicate exosphere variability modulated along the orbit, related only to seasonal variations and with a vertical profile purely consistent with a photon stimulated desorption release process (see Fig. 22b) (Cassidy et al. 2015). Kameda et al. (2009) measured the $\mathrm{Na}$ full-disk intensity from ground observations and confirmed a small short-time-scale modulation; they suggested a relation between the average intensity modulation and the crossing of the interplanetary dust disk.

Another disagreement between the MESSENGER data and the ground-based observations is the lack of evidence of a North-South asymmetry in the Na double peak distributions, obtained from the statistical analysis of the $\mathrm{Na}$ observations with the THEMIS telescope (Mangano et al. 2015). This fact may indicate that there is not any significant dipole shift as indicated by the MESSENGER data (Anderson et al. 2011b). Possible effects of higher order magnetic dipole harmonics could have a role (Richer et al. 2012), since a quadrupole component could produce a similar magnetosphere configuration in the northern hemisphere, even if significant differences should be present in the Southern magnetosphere. These two cases could not be discriminated in the MESSENGER data, since the spacecraft was in a high-eccentricity orbit with periherm close to the north pole (Solomon et al. 2007), obtaining images of the planet's exosphere through limb observations at the equatorial latitude. 
The distributions of other species, like $\mathrm{Mg}$ and $\mathrm{Ca}$, as observed by MESSENGER, peak at dawn, consistently with a release induced by micrometeoroid vaporization, assuming a preferential precipitation in the leading side (Burger et al. 2014). Ca seasonal variation follows the behaviour expected from impact vaporization by interplanetary dust, due to Mercury's substantial radial excursions (heliocentric distances between $0.306 \mathrm{AU}$ at perihelion and $0.465 \mathrm{AU}$ at aphelion). However, impact vaporization due to a single dust disk cannot explain all the variations seen in Mercury's Ca source rate; the strong peak in the $\mathrm{Ca}$ signal near TAA $=25^{\circ} \pm 5^{\circ}$ requires an additional source of dust. It can be attributed to the crossing of Mercury's orbital plane and a comet stream (Killen \& Hahn 2015).

The exospheric particles move independently from each other in a non-collisional regime; nevertheless, they do not move along simple ballistic trajectories: in fact, the alkalireleased particles are subject to radiation pressure that pushes them in anti-sunward direction, thus producing the tail observed in $\mathrm{Na}$ and in $\mathrm{K}$ (Potter et al. 2002).

Furthermore, some particles like oxides can be released as molecules, and impact vapour can remain in the uncondensed state after the initial fireball becomes collisionless (e.g. Berezhnoy \& Klumov 2008). The interaction with the solar UV radiation can dissociate the molecules, thus increasing their temperature. Such processes can explain the high temperature observed in $\mathrm{Ca}$ and $\mathrm{Mg}$ exospheric profiles (Killen et al. 2005).

Also the UV photons can ionize the exosphere, thus generating an ion population of planetary origin at low energy, not dense enough to be considered an ionosphere, that can be further accelerated and circulate inside the magnetosphere (Delcourt et al. 2003; Seki et al. 2013), as recently observed by MESSENGER (Raines et al. 2013). The absence of a conducting ionosphere implies that any field-aligned currents must close through the planet's surface. At a further step, the ions are either lost into the solar wind or impact again onto the surface producing a new generation of released material.

As mentioned before, the knowledge of the releasing process able to produce escape of material from the planet at present is crucial for understanding the long-term evolutionary scenario. In fact, in ancient times some of the release mechanisms were much more intense than today, due to different Sun conditions. As a matter of fact, according to Orsini et al. (2014), under the present conditions the surface material loss rate seems not to be able to produce a significant erosion of the planetary surface; in the past, instead, the long-term effects over billions of years could have affected the surface evolution of the planet in the context of the evolution of the Solar System. Following the timeline of the Solar System's history drawn by Coradini et al. (2011), Mercury formed in the Primordial Solar System before the end of the Late Heavy Bombardment (LHB, i.e., between 3.9 and 3.7 Ga ago), which marked the transition to the Modern Solar System, a phase lasting until now and characterized by less violent and more regular, secular processes (Coradini et al. 2011). Both the Sun's temperature and global luminosity increased vs. time (Guinan $\&$ Ribas 2002), hence in early phases the particle emission due to thermal desorption was not capable of producing significant erosion rates. The UV radiation at $100-360 \AA$ wavelengths is able to desorb $\mathrm{Na}$ and $\mathrm{K}$ from the planet's surface through Photon Stimulated Desorption (PSD; Killen et al. 2007). UV radiation was much more intense in early phases, so that it could have been able to remove volatile species, like $\mathrm{Na}$ and $\mathrm{K}$, and hence cause volatile depletion of the crust of Mercury down to depths of a few kilometres (during the Sun's T-Tauri phase), given that eruptive or bombardment-related mechanisms could have been able to extract such volatiles from underground (Orsini et al. 2014). The present $\mathrm{Na}$ abundance on Mercury's surface, with respect to the cosmic original abundance, could be explained by the effectiveness of this extraction from underground and subsequent PSD release. Moreover, both solar wind velocity and density significantly decreased from ancient times to today (Lundin et al. 2007). Ion precipitation on the planet's surface generates material emission through ion sputtering processes, producing a significant portion of material able to escape from Mercury (Wurz \& Lammer 2003; Mura et al. 2006). According to Orsini et al. (2014), the estimated erosion due to ion sputtering amounts to $10 \mathrm{~m}$ across the last $4.4 \mathrm{Ga}$, and $1 \mathrm{~m}$ since the end of LHB. Even if the LHB would have erased most of the pre-existing geological features, the overall loss rate from the surface of Mercury, via all Sun-related surface erosion and volatile depletion processes, is an important element for the interpretation of the surface features.

\section{Comparative planetology considerations}

\subsection{A synthetic view of space weather all over the Solar System}

Based on the discussions in Section 2, it is clearly understood that the different characteristics of the plasma-body interactions result in different space weather conditions at different locations in the Solar System. Whereas space weather in the inner Solar System is determined predominantly by the energy release from the Sun, in the form of solar photon flux, solar wind, CMEs and SEPs, space weather in the outer Solar System is driven by the balance between the incoming energy of solar origin and the internal sources in the form of plasma, neutral particles and fast planetary rotation. A typical example of such a process takes place is Jupiter's ionosphere: whereas the main auroral emission is controlled by Io's volcanism and the fast planetary rotation (Cowley \& Bunce 2001), it is also influenced by solar wind compressions (Nichols et al. 2007).

In Table 2 we summarize the characteristics of different environments in the Solar System related to planetary space weather. Space weather phenomena in planetary systems have their origin in plasma and energetic particle sources, whereas their development is strongly conditioned by the planetary bodies' magnetic fields (where present). Plasma populations can be of solar, planetary or satellite origin and they can be described in general by Maxwellian distributions, which often show non-Maxwellian suprathermal tails decreasing as a power law in velocity (kappa distributions, Vasyliunas 1968). The energetic particle populations have energies in the $\mathrm{keV}-\mathrm{GeV}$ range and originate either from sources external to the planetary system (e.g. GCR, SEPs) or from plasma acceleration mechanisms within the system. On the basis of their energy distribution and ion composition, the internal plasma sources in a planetary system (e.g. exosphere, ionosphere, moons, rings) can be distinguished, in general, from the external ones (e.g. solar wind, SEP, cosmic rays) (Krupp 2015). Nevertheless, Mauk (2014), through a 
Table 2. Different environments in the Solar System and their characteristics related to planetary space weather. In order to characterize the effects of solar emissions and winds on different bodies, we consider the existence of ionosphere (I), magnetosphere (M), exosphere (E). The interplanetary (or planetary) magnetic field inclination, given in the Table's last column, is measured with respect to the body's spin axis; the estimations of the IMF inclination are based on the Parker model, for an average solar wind velocity equal to $450 \mathrm{~km} / \mathrm{s}$

\begin{tabular}{|c|c|c|c|c|c|c|c|c|c|}
\hline \multicolumn{10}{|c|}{ Planets } \\
\hline \multirow[t]{2}{*}{ Solar system body } & \multirow[t]{2}{*}{$\begin{array}{l}\text { Distance from } \\
\text { the Sun (in } A U \text { ) }\end{array}$} & \multirow[t]{2}{*}{$\begin{array}{l}\text { Body's radius (in } \\
\text { km) }\end{array}$} & \multirow{2}{*}{$\begin{array}{l}\text { Rotation } \\
\text { period (in } \\
\text { hours) }\end{array}$} & \multirow{2}{*}{$\begin{array}{l}\text { Planetary } \\
\text { space } \\
\text { weather } \\
\text { regions }\end{array}$} & \multirow[t]{2}{*}{$\begin{array}{l}\text { Atmosphere major } \\
\text { composition }\end{array}$} & \multirow{2}{*}{$\begin{array}{l}\text { Magnetic } \\
\text { field } \\
\text { morphology }\end{array}$} & \multirow[t]{2}{*}{$\begin{array}{l}\text { Solar wind density } \\
\quad\left(\text { in } \mathrm{cm}^{-3} \text { ) }\right.\end{array}$} & \multicolumn{2}{|c|}{$\begin{array}{c}\text { Interplanetary magnetic } \\
\text { field }\end{array}$} \\
\hline & & & & & & & & $\begin{array}{l}\text { Intensity } \\
\text { (nT) }\end{array}$ & $\begin{array}{l}\text { Inclination } \\
\text { (in deg) }\end{array}$ \\
\hline Neptune & $30.07^{1}$ & $24,764^{1}$ & $16.11^{1}$ & $\mathrm{I}, \mathrm{M}, \mathrm{E}$ & $\mathrm{H}_{2}, \mathrm{He}, \mathrm{CH}_{4}{ }^{1}$ & Dipole & $0.008^{15}$ & $0.11^{22}$ & 88.1 \\
\hline Uranus & $19^{1}$ & $25,559^{1}$ & $17.24^{1}$ & $\mathrm{I}, \mathrm{M}, \mathrm{E}$ & $\mathrm{H}_{2}, \mathrm{He}, \mathrm{CH}_{4}^{1}$ & Multipole & $0.02^{15}$ & $0.22^{23}$ & 87.0 \\
\hline Saturn & $9.5^{1}$ & $60,330^{1}$ & $10.656^{1}$ & $\mathrm{I}, \mathrm{M}, \mathrm{E}$ & $\mathrm{H}_{2}, \mathrm{He}^{1}$ & Dipole & $0.1^{15}$ & $0.2-0.8^{24}$ & 83.9 \\
\hline Jupiter & $5.2^{1}$ & $71,398^{1}$ & $9.925^{1}$ & I, M, E & $\mathrm{H}_{2}, \mathrm{He}^{1}$ & Dipole & $0.3^{15}$ & $0.5-1.21^{25}$ & 79.0 \\
\hline Mars & $1.524^{1}$ & $3,390^{1}$ & $24.623^{1}$ & $\mathrm{I}, \mathrm{E}$ & $\mathrm{CO}_{2}, \mathrm{~N}_{2}{ }^{1}$ & Crustal fields & $3.5^{15}$ & $2-3^{26}$ & 56.4 \\
\hline Venus & $0.723^{1}$ & $6,051^{1}$ & $5,832.6^{1}$ & $\mathrm{I}, \mathrm{E}$ & $\mathrm{CO}_{2}, \mathrm{~N}_{2}{ }^{1}$ & Induced & $16^{15}$ & $12^{27}$ & 35.5 \\
\hline Mercury & $0.31-0.47^{1}$ & $2,439^{1}$ & $1,407.6^{1}$ & $\mathrm{M}, \mathrm{E}$ & $\begin{array}{c}\text { Exosphere: } \mathrm{Na} \text {, } \\
\mathrm{K}, \mathrm{Mg}, \mathrm{Ca}, \mathrm{H}, \\
\mathrm{He}, \mathrm{Al}^{1}\end{array}$ & Dipole & $32-73^{16}$ & $15-30^{28}$ & 21.1 \\
\hline
\end{tabular}

\begin{tabular}{|c|c|c|c|c|c|c|c|c|c|c|}
\hline \multicolumn{2}{|c|}{ Solar system body } & \multirow{2}{*}{$\begin{array}{l}\text { Distance from } \\
\text { the parent planet } \\
\text { (in planet radii) }\end{array}$} & \multirow[t]{2}{*}{$\begin{array}{l}\text { Body's radius (in } \\
\text { km) }\end{array}$} & \multirow{2}{*}{$\begin{array}{l}\text { Rotation } \\
\text { period (in } \\
\text { Earth } \\
\text { days) }\end{array}$} & \multirow{2}{*}{$\begin{array}{l}\text { Planetary } \\
\text { space } \\
\text { weather } \\
\text { regions }\end{array}$} & \multirow{2}{*}{$\begin{array}{l}\text { Major composition of } \\
\text { the neutral } \\
\text { environment }\end{array}$} & \multirow{2}{*}{$\begin{array}{l}\text { Magnetic } \\
\text { field } \\
\text { morphology }\end{array}$} & \multirow{2}{*}{$\begin{array}{l}\text { Magnetospheric } \\
\text { plasma density (in } \\
\mathrm{cm}^{-3} \text { ) }\end{array}$} & \multicolumn{2}{|c|}{$\begin{array}{l}\text { Planet's magnetic field at } \\
\text { the satellite's distance }\end{array}$} \\
\hline & & & & & & & & & $\begin{array}{l}\text { Direction } \\
\text { and } \\
\text { intensity } \\
\text { (nT) }\end{array}$ & Inclination \\
\hline \multirow[t]{4}{*}{$\begin{array}{l}\text { Jupiter's } \\
\text { satellites }\end{array}$} & Io & $5.91^{2}$ & $1,821.6^{2}$ & S $1.77^{2}$ & $\mathrm{E}$ & Exosphere: $\mathrm{SO}_{2}{ }^{4}$ & - & $\begin{array}{l}1,920 \text { (ions) } 2,500 \\
\text { (electrons) }^{17}\end{array}$ & $-1,900^{29}$ & 32,33 \\
\hline & Europa & $9.40^{2}$ & $1,560.8^{2}$ & S $3.55^{2}$ & $\mathrm{E}$ & $\begin{array}{l}\text { Exosphere: } \mathrm{O}_{2}, \mathrm{H}_{2} \mathrm{O} \\
\text { gas plume }\end{array}$ & Induced $^{12}$ & $\begin{array}{c}8.8-40.6\left(\mathrm{~S}^{2+}\right) \\
\left(\mathrm{O}^{2+}\right) 7.6-23.2 \\
-290 \text { (electrons) })^{18}\end{array}$ & $-420^{29}$ & 32,33 \\
\hline & Ganymede & $14.97^{2}$ & $2,631.2^{2}$ & S $7.15^{2}$ & M, E & Exosphere: $\mathrm{O}_{2}, \mathrm{H}^{6}$ & $\begin{array}{l}\text { Intrinsic } \\
\text { dipole and } \\
\text { induced }^{13}\end{array}$ & $\begin{array}{c}1-8 \text { (ions) } 1-10 \\
\text { (electrons) }^{17}\end{array}$ & $-90^{29}$ & 32,33 \\
\hline & Callisto & $26.33^{2}$ & $2,410.1^{2}$ & S $16.69^{2}$ & $\mathrm{E}$ & Exosphere: $\mathrm{CO}_{2}, \mathrm{O}_{2}{ }^{7}$ & Induced $^{12}$ & $\begin{array}{l}0.10 \text { (ions) } 0.15 \\
\quad(\text { electrons })^{17}\end{array}$ & $-30^{29}$ & 32,33 \\
\hline \multirow[t]{4}{*}{$\begin{array}{l}\text { Saturn's } \\
\text { satellites }\end{array}$} & Enceladus & $3.95^{3}$ & $257 \times 251 \times 248^{3}$ & S $1.37^{3}$ & $\mathrm{E}$ & $\begin{array}{c}\text { Exosphere (gas } \\
\text { plume): } \mathrm{H}_{2} \mathrm{O}, \mathrm{CO}_{2}, \\
\text { Mass } 28\left(\mathrm{CO}^{8} \text { or } \mathrm{N}_{2}\right) \\
\left.\mathrm{CH}_{4}{ }^{8}\right)\end{array}$ & $?^{14}$ & $\begin{array}{l}\text { (ions) } \\
\text { (electrons) }\end{array}$ & $-370^{30}$ & 32,34 \\
\hline & Dione & $6.26^{3}$ & $563 \times 561 \times 560^{3}$ & S $2.74^{3}$ & $\mathrm{E}$ & Exosphere: $\mathrm{O}_{2}, \mathrm{CO}_{2}{ }^{9}$ & $?^{14}$ & $\begin{array}{l}13 \text { (electrons) } \\
\text { (ions) }^{19}\end{array}$ & $-75^{30}$ & 32,34 \\
\hline & Rhea & $8.74^{3}$ & $765 \times 763 \times 762^{3}$ & $\mathrm{~S} 4.52^{3}$ & $\mathrm{E}$ & Exosphere: $\mathrm{CO}_{2}, \mathrm{O}_{2}{ }^{10}$ & $?^{14}$ & $\begin{array}{l}2 \text { (electrons) } \\
\text { (ions) })^{0.4-3}\end{array}$ & $-25^{30}$ & 32,34 \\
\hline & Titan & $20.27^{3}$ & $2,575^{3}$ & S $15.95^{3}$ & I, E & $\begin{array}{l}\text { Atmosphere: } \mathrm{N}_{2}, \\
\mathrm{CH}_{4}{ }^{11}\end{array}$ & Induced $^{14}$ & $\begin{array}{c}30-1,000 \text { (electrons) } \\
20-2,000 \text { (ions) }\end{array}$ & $-5.1^{31}$ & 32,34 \\
\hline
\end{tabular}


${ }_{1}^{1}$ As reported in NASA Planetary Fact Sheet http://nssdc.gsfc.nasa.gov/planetary/factsheet/

${ }^{2}$ From the NASA Jovian Satellite Fact Sheet http://nssdc.gsfc.nasa.gov/planetary/factsheet/joviansatfact.html; Note that S stands for synchronous rotation.

${ }^{3}$ From the NASA Saturnian Satellite Fact Sheet http://nssdc.gsfc.nasa.gov/planetary/factsheet/saturniansatfact.html; Note that S stands for synchronous rotation.

${ }^{4}$ As reported in Lopes \& Williams (2005).

5 As reported in Lopes \& Williams (2005).

${ }^{5}$ For observations interpreted as indicative for an $\mathrm{O}_{2}$ atmosphere see Hall et al. (1995, 1998), Saur et al. (2011) and McGrath et al. (2004). Observations interpreted as indicative for a transient water plume were discussed in Roth et al. (2014a).

${ }^{6}$ See Hall et al. (1998), Feldman et al. (2000), Barth et al. (1997).

${ }^{7}$ See Carlson (1999), Cunningham et al. (2015).

${ }^{8}$ See Waite et al. (2006).

${ }^{9}$ See Tokar et al. (2012), Teolis \& Waite (2016).

${ }^{10}$ See Teolis et al. (2010).

${ }_{11}^{11}$ See Waite et al. (2004), Coustenis et al. (2010).

12 For Europa see Khurana et al. (1998, 2009). For Callisto see Khurana et al. (1998).

${ }^{13}$ See Kivelson et al. (1996).

${ }^{14}$ It is currently not known if induced magnetic fields are generated at the inner Saturnian moons. Titan's ionosphere, being subject to direct erosion by the incident plasma ?ow, generates an induced magnetosphere around the moon: the ambient magnetospheric ?eld drapes around Titan' s ionosphere, leading to the formation of a magnetic pile-up region at the ramside and a

bipolar magnetotail in the wake region (see Simon et al. 2013b for details)

${ }_{16}$ As reported in Richardson et al. (2004).

${ }_{17}$ As reported in Milillo et al. (2005).

${ }_{17}^{17}$ As reported in Kivelson et al. (2004, Table 21.1).

${ }^{18}$ For a detailed description of the plasma environment at Europa see Bagenal et al. (2015) and Delamere et al. (2005).

¿ See Sittler et al. (2006).

¿ $\quad{ }_{21}^{20}$ See Edberg et al. (2010).

స 22 See Mandt et al. (2012).

${ }^{23}$ See Voigt et al. (1983).

${ }^{24}$ See Jackman et al. (2004). These values were measured by Cassini in 2004 and correspond to magnetopause crossings.

${ }^{25}$ Ulysses data $(1992,1998,2004)$ and Cassini data (2000) taken from Table 1 in Nichols et al. (2006).

${ }^{26}$ Estimation based on the analysis of Mars Global Surveyor (MGS) Magnetometer (MAG) data from the region upstream from Mars (Brain et al. 2003). The spiral angle was found in the range $32^{\circ}-70^{\circ}$.

${ }^{27}$ See Luhmann et al. (1986).

${ }^{28}$ See Figure 5 in Baker et al. (2013). CME passages (with stronger magnetic fields in and draped around the CMEs) and other transient solar wind can result in IMF enhancements (Baker et al. 2013).

${ }^{29}$ Since neither the orbits nor the spin axes of the moons are significantly inclined to the parent planet's equatorial plane, we present here the averages around the moon's orbit as reported in Kivelson \& Bagenal (2007). The minus sign indicates that the average field over a planetary rotation period is southward oriented, i.e. antiparallel to the planet's rotation axis.

${ }^{30}$ See Khurana et al. (2008).

31 See Kivelson \& Bagenal (2007).

32 The magnetic fields of Jupiter and Saturn at the orbits of their moons oscillate in intensity and direction at the giant planets rotation periods, equal to $9.925 \mathrm{~h}$ and $10.656 \mathrm{~h}$, respectively.

${ }^{33}$ The average field over a planetary rotation period is southward oriented, i.e. antiparallel to Jupiter's axis of rotation (Kivelson \& Bagenal 2007). The spin axes of the moons are not significantly inclined to the parent planet's equatorial plane: $0.04^{\circ}$ (Io); $0.47^{\circ}$ (Europa); $0.21^{\circ}$ (Ganymede); $0.51^{\circ}$ (Callisto), according to http://nssdc.gsfc.nasa.gov/planetary/factsheet/ joviansatfact.html. Therefore the inclination of the parent planet's magnetic field (with respect to the satellite's spin axis) is negligible.

${ }^{34}$ As in the Jupiter's case, the spin axes of the moons are not significantly inclined to the parent planet's equatorial plane: $0.00^{\circ}$ (Enceladus); $0.02^{\circ}$ (Dione); $0.35^{\circ}$ (Rhea); $0.33^{\circ}$ (Titan), according to http://nssdc.gsfc.nasa.gov/planetary/factsheet/saturniansatfact.html. Therefore the inclination of the parent planet's magnetic field (with respect to the satellite's spin axis) is negligible. 
comparative analysis for Earth, Jupiter, Saturn, Uranus and Neptune, showed that the ion composition and energy spectra are subject to interaction processes in the magnetospheres dependent on the strength of plasma sources. This means that the space weather conditions at these systems are dynamically determined by interactions hence any attempt to identify the related phenomena, to study their potential impact on electronics and systems on-board spacecraft, and to develop a concept of forecasting, should begin from an in-depth study of the plasma source and loss processes. Laurenza et al. (2009) have recently developed a technique for obtaining a short-term warning of SEP events, based on the flare location, flare size and evidence of particle escape. Moreover, an extended review of the plasma-related processes in the Solar System has been provided by Seki et al. (2015).

Below, we briefly discuss the agents of planetary space weather from a comparative planetology point of view.

\subsubsection{Plasma and magnetic fields}

Numerous studies in the solar-terrestrial science field have essentially enriched our knowledge on the interaction between plasma of solar origin and the Earth, a strongly magnetized planet. The Earth's intrinsic magnetic field deflects the solar wind at the magnetopause location, a region where magnetic reconnection is likely to occur allowing the solar plasma entry in the Terrestrial magnetosphere.

The mechanisms through which the solar wind enters a planetary magnetosphere determine at first place the characteristics of the space weather phenomena in that environment. The solar wind-magnetosphere dynamics depend on one hand on the solar wind density, flow speed and IMF intensity in the vicinity of the body, and on the other hand on the magnetic obstacle due to the planet's own magnetic field. A necessary condition for reconnection is that the IMF has an anti-parallel component to the planetary magnetic field at this location. The angle between the IMF and the radial direction varies with increasing distance from the Sun, ranging from $\sim 20^{\circ}$ at Mercury's orbital distance of $\sim 0.4$ AU (Kabin et al. 2000) to $\sim 83^{\circ}$ at Saturn's orbital distance of $\sim 9.5$ AU (Jackman et al. 2008); the IMF strength varies with the inverse square distance from the Sun (see Table 2). Through the IMF-planetary magnetic field reconnection, mass, energy and momentum from the solar wind can be transferred into the planetary magnetosphere.

The rate of the magnetopause reconnection is determined by both the strength and the orientation of the IMF with respect to the planet's magnetic field, and the plasma conditions in the magnetosheath adjacent to the magnetopause (Seki et al. 2015). For the Earth, this is due to the high average Alfvenic Mach number at $1 \mathrm{AU}$ (Slavin et al. 1984) resulting in high plasma- $\beta^{6}$ magnetosheath and thin, weak plasma depletion layers (Zwan \& Wolf 1976). Nevertheless, other Solar System planets possessing an intrinsic magnetic field (such as Mercury, Jupiter and Saturn) are characterized by different plasma conditions and magnetic field strength, thus reconnection rate is likely to differ from the terrestrial magnetosphere case (Slavin et al. 2014). Typically high plasma- $\beta$ magnetosheaths at the Earth and outer planets cause the magnetic fields on either side of the magnetopause to differ largely in magnitude

\footnotetext{
6 The plasma- $\beta$ parameter is defined as the ratio of plasma thermal to magnetic pressure.
}

hence reconnection is only possible for large shear angles ${ }^{7}$ (Sonnerup 1974). On the contrary, for low plasma- $\beta$ magnetosheaths and well-developed plasma depletion layers (as it happens at Mercury Gershman et al. 2013), the similar in magnitude magnetic fields on either side of the magnetopause allow reconnection even for low shear angles (DiBraccio et al. 2013; Slavin et al. 2014). On the basis of MESSENGER observations, it has been found that the rate of reconnection at Mercury's magneopause is independent of the IMF direction (DiBraccio et al. 2013), being on average three times larger than its value at Earth (Slavin et al. 2009). During encounters with CMEs, however, the upstream Mach number at $1 \mathrm{AU}$ obtains values similar to the ones at Mercury and similar effects are expected (Lavraud et al. 2013). Based on a combination of Cassini spacecraft observations and simulations at Saturn, Masters et al. (2012) argued that plasma- $\beta$ conditions adjacent to Saturn's magnetopause largely restrict reconnection to regions of the boundary where the adjacent magnetic fields are close to anti-parallel, giving some evidence that the magnetopause surface that can become open may be limited. They showed that under relatively low magnetosheath $\beta$ conditions this restriction becomes less severe, demonstrating in this way that the nature of solar wind-magnetosphere coupling via reconnection can vary between planets and thus may not always be Earth-like. In this context, it becomes clear that the variability of the interplanetary magnetic field conditions determines, dynamically: (a) the spatial extension of the reconnection site (starting point of the space weather activity); (b) the reconnection rate (a parameter that is strongly related to the further evolution of the space weather activity).

Other important physical processes favouring the solar wind entry in a planetary magnetosphere are the anomalous diffusion across the magnetopause, caused by the KelvinHelmoltz instability, and the kinetic Alfvén waves. The Kelvin-Helmoltz instability is an MHD instability driven by a flow shear between the magnetosheath and the magnetosphere (Seki et al. 2015). Hasegawa et al. (2004a) noted that the plasma content in the outer terrestrial magnetosphere increases during northward solar wind magnetic field conditions (Mitchell et al. 1987; Hasegawa et al. 2004b), contrary to what would be expected in case reconnection were dominant. Hasegawa et al. (2004b) showed that during northward solar wind magnetic field conditions - in the absence of active reconnection at low latitudes - there is a solar wind transport mechanism associated with the nonlinear phase of the Kelvin-Helmholtz instability, supplying plasma sources for various space weather phenomena in the circum-terrestrial space.

A comparison of the parameters determining the characteristics of plasma sources in different magnetospheres of our Solar System is found in Bagenal (2013). We summarize the main characteristics of the Solar System planetary magnetospheres, related to space weather in Table 3. As shown in Table 3, the magnetospheric circulation is driven primarily either by the solar wind or by the planet's rotation. Within the same planetary system, the fraction of the magnetosphere that corotates in the region up to a critical distance from the planet, $R_{\mathrm{c}}$, is given by $R_{\mathrm{c}} / R_{\mathrm{mp}}=\left(R_{\mathrm{mp}} \Omega / \eta V_{\mathrm{sw}}\right)^{1 / 2}$, where $R_{\mathrm{mp}}$ is the magnetopause distance, $\Omega$ is the planet's rotation velocity, $\eta$ is the efficiency of the reconnection process in

\footnotetext{
${ }^{7}$ The shear angle is defined as the rotation of the magnetic field from the magnetosheath into the magnetosphere (DiBraccio et al. 2013).
} 
Table 3. Characteristic parameters of Solar System magnetospheres determining planetary space weather. Adapted from Bagenal (2013) and Krupp (2015).

\begin{tabular}{|c|c|c|c|c|c|c|c|c|c|}
\hline $\begin{array}{l}\text { Solar } \\
\text { System } \\
\text { body }\end{array}$ & $\begin{array}{l}\text { Magnetopause } \\
\text { nose distance }\end{array}$ & $\begin{array}{l}\text { Surface magnetic field } \\
\text { at the equator }(\mathrm{T})\end{array}$ & $\begin{array}{c}\text { Dipole } \\
\text { tilt }^{1}\end{array}$ & Obliquity $^{2}$ & "Plasma sources ${ }^{3}$ & $\begin{array}{c}\text { Plasma } \\
\text { composition (main } \\
\text { species) }\end{array}$ & Plasma motion & $\begin{array}{c}\text { Plasma } \\
\beta^{4}\end{array}$ & Lifetime \\
\hline Neptune & $24 \mathrm{R}_{\mathrm{N}}$ & $0.14 \times 10^{-4}$ & $-47^{\circ}$ & $29.6^{\circ}$ & Triton (solar wind?) & $\mathrm{N}^{+}, \mathrm{H}^{+}$ & $\begin{array}{c}\text { Rotation (and solar wind } \\
\text { driven?) }\end{array}$ & 0.2 & days \\
\hline Uranus & $25 \mathrm{R}_{\mathrm{U}}$ & $0.23 \times 10^{-4}$ & $-59^{\circ}$ & $97.9^{\circ}$ & Ionosphere (solar wind) & $\mathrm{H}^{+}$ & $\begin{array}{c}\text { Solar wind driven and } \\
\text { rotation }\end{array}$ & 0.1 & $\begin{array}{l}1-30 \\
\text { days }\end{array}$ \\
\hline Saturn & $19 \mathrm{R}_{\mathrm{S}}$ & $0.22 \times 10^{-4}$ & $-0^{\circ}$ & $26.7^{\circ}$ & $\begin{array}{l}\text { Enceladus (Rings, Tethys, } \\
\text { Dione, Titan, solar wind) }\end{array}$ & $\mathrm{H}_{2} \mathrm{O}^{+}, \mathrm{O}^{+}, \mathrm{H}^{+}$ & Rotation & $1-5$ & $\begin{array}{c}30-50 \\
\text { days }\end{array}$ \\
\hline Jupiter & $42 \mathrm{R}_{\mathrm{J}}$ & $4.28 \times 10^{-4}$ & $-9.6^{\circ}$ & $3.1^{\circ}$ & Io, Europa (ionosphere) ${ }^{7}$ & $\mathrm{O}^{\mathrm{n}+}, \mathrm{S}^{\mathrm{n}+}, \mathrm{H}^{+}$ & Rotation & $10-100$ & $\begin{array}{c}20-80 \\
\text { days }\end{array}$ \\
\hline $\begin{array}{l}\text { Mercury } \\
\text { Ganymede }\end{array}$ & $\begin{array}{l}1.4-1.6 \mathrm{R}_{\mathrm{M}} \\
\quad \sim 2 \mathrm{R}_{\mathrm{G}}\end{array}$ & $\begin{array}{c}2 \times 10^{-7} \\
7.19 \times 10^{-75}\end{array}$ & $\begin{array}{l}+14^{\circ} \\
176^{\circ}\end{array}$ & $\begin{array}{c}0^{\circ} \\
0.2^{\circ}\end{array}$ & $\begin{array}{l}\text { Solar wind } \\
\text { Jupiter's magnetosphere } \\
\text { (Ganymede's exosphere) }\end{array}$ & $\mathrm{O}^{\mathrm{n}+}, \stackrel{\mathrm{H}^{+}}{\mathrm{S}^{\mathrm{n}^{+}}, \mathrm{H}^{+}}$ & $\begin{array}{l}\text { Solar wind driven } \\
\text { Corotation (Jupiter's } \\
\text { magnetosphere driven) }\end{array}$ & $\begin{array}{l}\sim 2^{8} \\
0.4^{9}\end{array}$ & $\begin{array}{l}\text { Minutes } \\
\text { Min to } \\
\text { hours }\end{array}$ \\
\hline
\end{tabular}

${ }^{1}$ Angle between the magnetic and rotation axes.

2 Inclination of the planet's equator to the orbit.

3 The dominant plasma sources are indicated. In parentheses we indicate the believed secondary plasma sources.

${ }_{5}^{4}$ The plasma $\beta$ parameter is defined as the ratio of plasma pressure to magnetic pressure, $\beta=n \mathrm{kT} /\left(B^{2} / 2 \mu_{0}\right)$.

${ }_{6}^{5}$ From Kivelson et al. (1996).

${ }^{6}$ Blanc et al. (2015).

${ }^{7}$ Bolton et al. (2015)

${ }^{8}$ Raines et al. (2014).

${ }^{9}$ Temporal analysis of the auroral power of Ganymede's footprint reveals variations of different timescales: (a) a 5-h timescale, associated with the periodic flapping of Jupiter's plasma sheet over Ganymede; (b) a 10-40 min timescale, possibly associated with energetic magnetospheric events, such as plasma injections; (c) a 100-s timescale corresponding to quasiperiodic fluctuations, which might relate to bursty reconnections on Ganymede's magnetopause and/or to the recurrent presence of acceleration structures above Jupiter's atmosphere. From Grodent et al. (2009). 
harnessing the solar wind momentum and $V_{\mathrm{sw}}$ is the velocity of the solar wind (Bagenal 1992). So, in the magnetospheres of rapidly rotating planets with strong magnetic fields (e.g. Jupiter, Saturn), plasma motions are dominated by rotation. A similarity among magnetospheres of this kind, with direct effects on space weather, is that their icy moons supply heavy ions such as watergroup species, oxygen, sulphur and nitrogen. Due to the fast planet rotation, such ions are concentrated near the centrifugal equator in a disk-like current sheet and associated plasma sheet, whereas, away from it the plasma density drops (Krupp 2015). In smaller magnetospheres around slowly rotating planets (e.g. Mercury), solar wind controls mainly the plasma motions. A major difference between the magnetospheres of Jupiter and Saturn compared with Uranus and Neptune, with impact on the actual interactions ruling space weather, is the plasma- $\beta$ value. For Uranus and Neptune, $\beta$ is in the range from $\sim 0.1$ to 0.2 , while for Saturn $\beta$ is in the range from $\sim 1$ to 5 and for Jupiter $\beta$ is in the range from $\sim 10$ to 100 (see also Table 3), meaning that the Jovian and the Saturnian magnetospheres are particle-driven and vice versa for Uranus and Neptune (Krupp 2015). Table 3 shows that in the magnetospheres where plasma circulation is driven by the solar wind, plasma of solar origin becomes an important source (the dominant one in case of Mercury). On the contrary, in the magnetospheres where the plasma circulation is mainly driven by the planetary rotation (e.g. Saturn, Jupiter) the internal plasma sources (e.g. the volcanic activity at Jupiter's moon Io and the plume activity at Saturn's moon Enceladus) determine in a significant way the characteristics of the interactions within the system. The magnetosphere of Neptune is a more complicated case since at the current moment it is not clear if solar wind could have an important role in driving the plasma motion, as it is believed in the case of Uranus. We note that the distribution of plasma at Neptune is generally interpreted as indicative of Triton acting as a major plasma source (Krimigis et al. 1989; Richardson et al. 1991).

At the moons of the giant planets the plasma-body interactions have significantly different characteristics with respect to those in bodies with or without magnetic field, depending on the electrical conductivity of the moons' ionospheres and the presence of exospheric neutrals that act as the interface between the environment external to the body and the surface. For example, in the vicinity of the Galilean moons, the formation of a shock bounding the upstream region has not been observed (Kivelson et al. 2004). This is due to the fact that in the vicinity of these bodies the MHD waves can transfer energy and momentum faster than the relative speed of Jupiter's magnetospheric plasma. Therefore, the fastest MHD waves can propagate upstream of a moon whereas the perturbations that slow and divert the flow develop gradually and shocks do not form, except possibly at Callisto (Kivelson et al. 2004). Moons are significant plasma sources for the outer planets' magnetospheres providing material through volcanism, plumes or particles released directly from their surfaces due to sputtering, radiolysis and sublimation (Cassidy et al. 2010; Plainaki et al. 2012, 2015). Io and Europa are believed to be major plasma sources for Jupiter's magnetosphere, similarly to Enceladus for Saturn's and Triton for Neptune's magnetospheres (see Table 3). Water plumes have been observed in-situ at Enceladus (Porco et al. 2014) and remotely at Europa, in December 2012 (Roth et al. 2014a). No further evidence for plumes was found during subsequent HST observations of Europa's auroral emission (Roth et al. 2014b). The transient nature of the plume phenomenon is expected to have impacts on the space weather interactions in the moon's exosphere (e.g. presence or absence of watergroup plasma ions). We note that the Voyager 2 data provided no evidence for major particle release from the icy surfaces of the Uranian moons, however the possibility cannot be ruled out (Mauk et al. 1987).

Interactions between plasma and moon undersurface oceans can provide evidence for the existence of such liquid oceans. This is the case of Jupiter's moon Europa (see Khurana et al. 2009 and references therein). However, the weak periodic variation of Saturn's field due to the near alignment of the planet's magnetic dipole axis with the spin axis (Smith et al. 1980; Connerney et al. 1981) does not allow the diagnosis of induction at the moons, as it happens at Jupiter. The strongest time-varying fields suitable for sounding Saturnian moons exist around those moons that enter the solar wind such as Iapetus (Dougherty et al. 2009). Future studies of space weather at these bodies, therefore, could provide some insights into magnetic induction.

When a planet has a global magnetic field or when a satellite is embedded into the magnetosphere of a planet (e.g. Ganymede's mini-magnetosphere embedded in Jupiter's magnetosphere), ionospheric ions can escape providing a source of plasma for the surrounding magnetosphere. Atmospheric escape is one dramatic effect of planetary space weather. At Uranus and probably Neptune the ionospheres play important roles in providing plasma to the magnetospheres while for Jupiter and Saturn only minor contributions for the overall budget of plasma are assumed to originate in the atmosphere/ionosphere of those planets (Krupp 2015). Exoplanets with short orbital separation from their stars lose also their atmospheres (Vidal-Madjar et al. 2003). At some satellites (e.g. Io) atmospheric escape creates a torus (Steffl et al. 2008). These outflowing ions along magnetic fields, observed many times in the past at different environments (for a review see Chappell 2015), influence the space weather conditions around the body in the Solar System and their measurement (when possible) can be used as a proxy for the actual interaction processes taking place within. Seki et al. (2015) summarized the most important types of ionospheric ion outflows from a magnetized planet or satellite with atmosphere, namely the polar wind, the bulk ion upflow, the ion conics and the ion beams. Especially the two latter categories are highly dependent on the external space conditions hence their variability determines in a dynamic manner the space weather conditions around the body. For example, ion conics (named after the typical shape of the velocity distribution function of ion outflows) are caused by transverse acceleration in terms of local magnetic field when there is an energy input such as electron precipitation into a planetary ionosphere under an open magnetic field line geometry (Seki et al. 2015). Equivalently, when the magnetospheric population has significant differential anisotropy between the ion distribution and the electron distribution, significant parallel potential drops can develop (Wu et al. 2002) accelerating electron either downwards, causing discrete auroras, or upwards creating ion outflows in the form of fieldaligned energetic beams.

\subsubsection{The role of flares and CMEs}

Solar wind disturbances can also have significant planetary space weather manifestations, as it happens in the circum-terrestrial space weather case. According to Lilensten 
et al. (2014) these disturbances are of two categories: (a) the solar wind stream interactions resulting in Parker spiral compression regions and rarefactions, and (b) Interplanetary CMEs (ICMEs), that is plasma structures propagating outward through the solar wind, causing the background plasma and field to pile up in front of them creating leading interplanetary shocks. Nevertheless, the planetary space weather effects produced by these two classes of solar wind disturbances are not identical. In fact, Slavin et al. (2014), investigating the structure of Mercury's dayside magnetosphere during ICMEs and high-speed stream events, identified different effects. In particular, ICME events at Mercury resulted in thick, low plasma- $\beta$ depletion layers and high reconnection rates of $0.1-0.2$, despite small magnetic shear angles across the magnetopause of only $27-60^{\circ}$. On the contrary, the high-speed stream event produced a high plasma- $\beta$ magnetosheath with no plasma depletion layer and large magnetic shear angles of $148-166^{\circ}$, but low reconnection rates of $0.03-0.1$ (Slavin et al. 2014). This study confirmed that the rate of magnetic reconnection at Mercury is primarily controlled by the dynamic space weather conditions in the planet's vicinity, which determine the plasma- $\beta$ in the adjacent magnetosheath. Moreover, similarly to the Earth case, the distance to the subsolar magnetopause is reduced during these solar wind disturbance events from its mean of 1.45 Mercury radii to between 1.03 and 1.12 Mercury radii (distances measured with respect to the planetary magnetic dipole position). We note that in most simple scenarios applied for the circum-terrestrial space weather case, the shock impacting the subsolar point of the magnetosphere drives the magnetopause earthward in a piston-like motion that launches a fast-mode wave that propagates both radially inward (earthward) and azimuthally around the Earth (Araki et al. 1997; Gannon et al. 2005). For the Earth case, Pudovkin et al. (1998), in a statistical study on the magnetopause compression dependence on solar wind parameters, showed that the observed magnetopause standoff distances vary by a factor up to $\sim 2$ depending on the orientation of the IMF field.

The study of the interplanetary propagation and arrival-time of a CME at a planet's vicinity can be done through the use of the ensemble WSA-ENLIL+Cone model from the Community Coordinated Modeling Center (CCMC; located at NASA Goddard Space Flight Center). The global 3D MHD ENLIL model provides a time-dependent description of the background solar wind plasma and magnetic field using the WSA coronal model (Arge \& Pizzo 2000; Arge et al. 2004) as input at the inner boundary of 21.5 solar radii (Odstrčil et al. 1996; Odstrčil \& Pizzo 1999a, 1999b; Odstrčil 2003; Odstrčil et al. 2004). A homogeneous, overpressured hydrodynamic plasma cloud is launched through the inner boundary of the heliospheric computational domain and into the background solar wind. The modelled CME cloud is approximated by a sphere, however ENLIL also supports other CME shapes such as an ellipsoid that can have an arbitrary tilt with respect to the solar equator. The outer radial boundary can be adjusted to include planets or spacecraft of interest (e.g. 2 AU to include both Earth and Mars, 5 AU to include Ulysses, $10 \mathrm{AU}$ to include Cassini). It covers $60^{\circ}$ north to $60^{\circ}$ south in latitude and $360^{\circ}$ in azimuth. Figure $23 \mathrm{~b}$ shows the propagation of a CME in the Internal Solar System, ejected from the Sun on 4 June 2011, as simulated through the WSA$\mathrm{ENLIL}+\mathrm{Cone}$ model; for comparison the situation during quiet solar conditions is also shown (Fig. 23a). Recently, the WSA-ENLIL+Cone model was applied for the case of
7 January 2014 CME event (Mays et al. 2015). We note that during favourable alignments of the planets, in-situ observations of the solar wind at Mercury, Venus, Earth and Mars provide valuable data to verify the model and develop better forecasts.

Impulsive and gradual SEP events (Reames 1999) are likely to affect the atmosphere of a planetary body in the inner Solar System, being associated with populations with energies ranging, typically, up to some hundreds of $\mathrm{MeVs} /$ nucleon, highly dependent on solar activity. Studies of the solarterrestrial physics have shown that on rare occasions, the SEPs arriving at the distance of $1 \mathrm{AU}$ from the Sun can have energies up to a few GeVs/nucleon (Gopalswamy et al. 2012). Depending on their velocity direction, these relativistic solar particles may overcome the Earth's magnetic cut-off at different latitudes and enter the Terrestrial atmosphere. ${ }^{8}$ Upon entry, they can produce atmospheric showers of secondary particles with sufficient energy to be detected by ground-level Neutron Monitor detectors and with intensities that exceed the GCR background (see among others: Storini et al. 2005; Belov et al. 2005; Cliver 2006; Bombardieri et al. 2008; Plainaki et al. 2007, 2010b; Mavromichalaki et al. 2011). The resulting ground-level enhancements (GLEs) of the cosmic ray intensity are a dramatic effect of the circum-terrestrial space weather, being related to the most energetic class of SEP events (Plainaki et al. 2014). SEP fluxes reaching Venus and Mars are of special interest because the atmospheres of these planets are poorly protected by the weak magnetic fields. Depending on the solar particle energy and direction, SEP events at Venus may result in increased ionization rates in the atmosphere (see estimations by Nordheim et al. 2015; Plainaki et al. 2016). The NASA MAVEN mission will provide feedback on the SEP interactions with Mars' upper atmosphere and on how they have contributed to its evolution over time. Since the increased electron flux impacting a planetary atmosphere can also initiate processes such as charge exchange and ionization, the variability in electron flux can be equivalently considered as an indicator for space weather action.

During solar flares, variation in the solar flux in the spectral range extending from the UV to the X-ray wavelengths can have a direct effect on the ionization of the atmospheres of planetary bodies as well as on their heating and dimensions (for details see Schunk \& Nagy 2009). Relatively long wavelength photons $(>90 \mathrm{~nm})$ cause, in general, dissociation, whereas shorter ones cause ionization (Seki et al. 2015), with efficiencies depending on the atmospheric species in each planetary environment. During flares, the solar irradiance at these energetic wavelengths can increase by one order of magnitude (Lilensten et al. 2014). For the terrestrial planets possessing an atmosphere the increased solar flux causes similar effects. At Venus the higher solar EUV fluxes produce a more extended neutral thermosphere and a denser exosphere, as well as an increase of the photoionization rate (Luhmann et al. 2007). The unusually dense Martian ionosphere on 15 and 26 April 2001, detected through enhancements in radio signals from the Mars Global Surveyor (MGS), was attributed to some extra production of ions and electrons after the occurrence of several flares. Similar modifications in the Earth's ionosphere at these times were also measured (we note that the Sun, Earth and Mars were nearly in a straight line at that time) (Lollo et al. 2012). We note that photoionization is the major source of ionization in sunlit ionospheres, providing well-defined

\footnotetext{
${ }^{8}$ The minimum cut-off energy is equal to $\sim 433 \mathrm{MeV} /$ nucleon.
} 

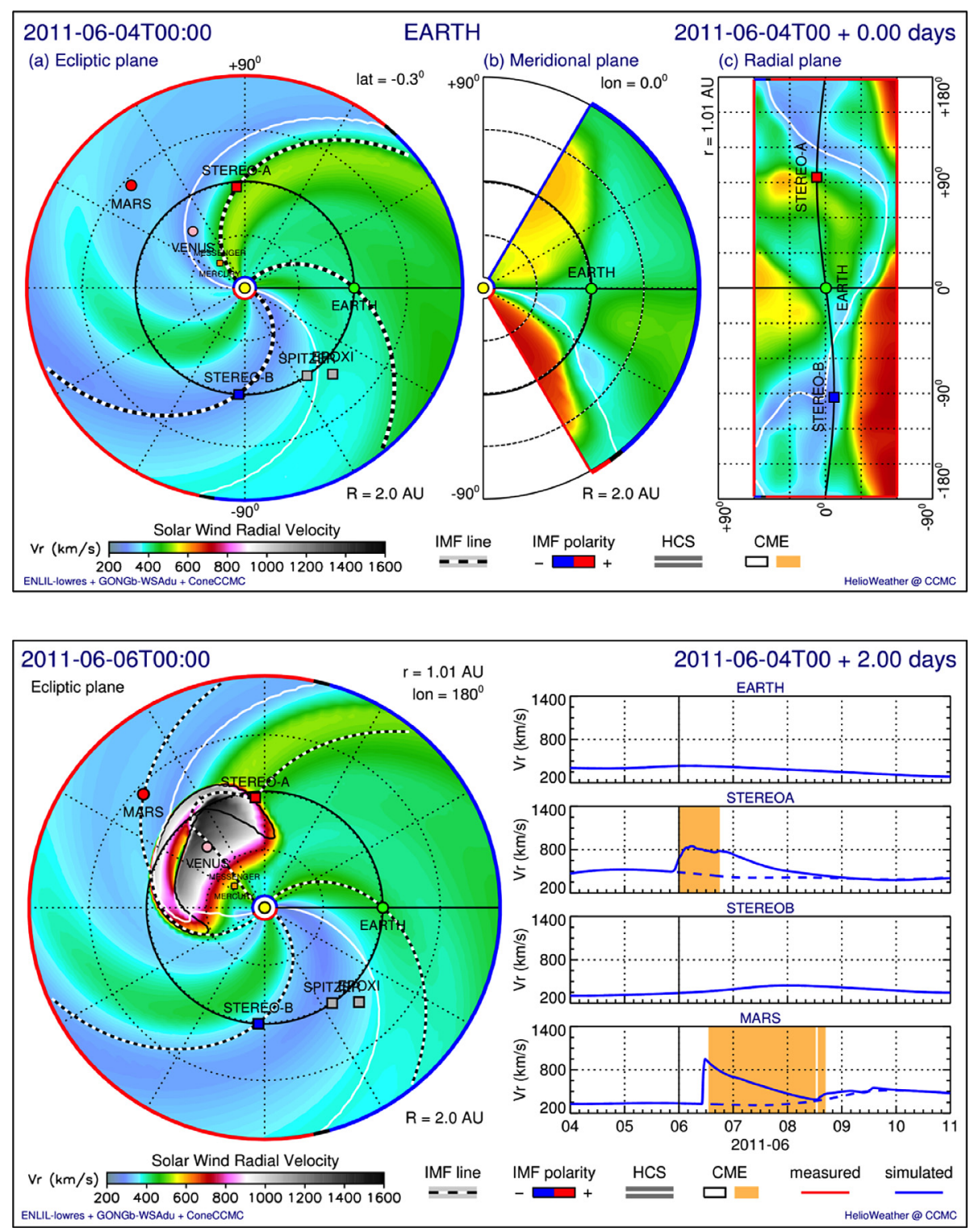

Fig. 23. Global view of the 4 June 2011 CME, on 6 June 2011 (lower panel) in comparison with the quiet solar conditions' situation (upper panel). (a) WSA - ENLIL + Cone radial velocity contour plot for the (a) Ecliptic plane, (b) meridional plane of Earth and (c) 1 AU sphere in cylindrical projection. (b) the global view of the CME on 6 June 2011 at the Ecliptic plane. The simulated radial velocity profiles at the Earth's, STEREO A, STEREO B and Mars positions are presented in the graph. The simulated CME duration is shown in yellow. The image is courtesy of Leila Mays.

unique features in the electron energy spectrum, which can be seen also at locations remote from where they are produced (Coates et al. 2011). As neutrals from the planetary atmospheres are ionized, ions and photoelectrons are being generated. Ionospheric photoelectrons can be used as an important diagnostic tool for the topology of the solar wind interaction with both magnetized and unmagnetized objects (Coates et al. 2011), possibly playing a role also in enhancing the ion escape. Ionospheric photoelectron peak features are also seen in Saturn's ring ionosphere (Coates et al. 2005) and in the neutral-rich inner magnetosphere of Saturn (Schippers et al. 2009) and they are predicted in Saturn's ionosphere (Galand et al. 2009).

\subsubsection{Energetic particle populations}

As evidenced in Sections 2.1.2.2 and 2.1.3.2, the energetic charged particles trapped in the radiation belts of the strongly magnetized planets give rise to space-planetary environment interactions. It is widely known that if the planetary magnetic field is non-uniform, the energetic ions and electrons drift in opposite directions around the planet creating an (azimuthal) ring current, which can provoke perturbations to the planet's magnetic field if the plasma $\beta$ parameter is high (e.g. Jupiter and Saturn, see also Table 3). Bagenal (1992) estimated that the charged particle energy builds up to only $1 / 1000$ of the magnetic field energy in each magnetosphere in the Solar System, independently from the different total energy content in each case. The energetic charged particle populations at Jupiter and Saturn are close to this limit (Connerney et al. 1983) whereas the radiation belts of Uranus and Neptune are much less than this limit (Connerney et al. 1991). The existence of high-energy protons in the inner radiation belts of Jupiter, Saturn, Uranus and Neptune is believed to be the result of the CRAND process (Kollmann et al. 2013; Bolton et al. 2015; Krupp 2015). 
The charged energetic particles in planetary magnetospheres can be related to space weather, in either a direct or an indirect way.

Directly: Charge-exchange between energetic ions and cold neutrals in planetary (or lunar) atmospheres or neutral tori results in the generation of fast ENAs which are no longer trapped in the planet's magnetic field. These ENAs leave the interaction region in ballistic orbits in the direction of the incident ion velocity at the time of the impact. Imaging their distribution allows us to investigate the structure of planetary magnetospheres (Brandt et al. 2001) and, therefore, to map the actual space weather process in action. The magnetospheres emit copious quantities of ENAs at energies between tens of eVs and hundreds of keVs, which carry significant amounts of energy and mass as found by Mauk et al. (2003). These authors analysed 50-80 keV ENA images of Jupiter's magnetosphere and identified two dominating emission regions: the upper atmosphere of Jupiter itself and a torus of emission residing just outside the orbit of Jupiter's satellite Europa. Scattering of the energetic particles by waves can direct them into the upper atmospheres of planets where they can excite auroral emission (Cheng et al. 1987).

Indirectly: The impact of energetic ions on an icy satellite surface is followed by the release of surface material to the body's exosphere through direct sputtering and/or radiolysis (for an example of how these release mechanisms work see: Johnson et al. 2004; Cassidy et al. 2010; Teolis et al. 2010; Plainaki et al. 2012). The generated exospheres are furthermore subject to loss processes either due to gravity escape and particle sticking to the surface or due to photon or plasma-induced ionization/dissociation of their constituents. The variability of both the energetic ions and plasma populations and possibly the body's illumination by the Sun (Plainaki et al. 2013) are responsible for the dynamic generation and loss of the moon exospheres. Space weather effects in these environments, consequently, are effectively seen in the spatial and energy distributions of the neutral particle densities at these moons. The variability of these distributions, therefore, can be an index for the effectiveness of space weather interactions within the whole system.

\subsubsection{The aurorae and the energy balance problem}

As evidenced in previous sections, airglow is a manifestation of planetary space weather. Auroral emissions, occurring when charged particles in a planetary object's magnetosphere collide with atoms in its upper atmosphere, causing them to glow, are an important demonstration of planetary space weather.

A comparison between the measured exospheric temperatures of the giant planets with those calculated from solar EUV inputs alone (see Table 4) showed that considerable additional energy sources are required to produce the temperatures observed in the giant planets (Miller et al. 2005). Several mechanisms strongly related to the variability of the environment of the charged populations at the giant planets have been proposed as possible energy sources resulting in the exospheric temperature increase (see Waite et al. 1983; Young et al. 1997; Grodent et al. 2001). Particle precipitation in Jupiter's auroral/polar regions is estimated to provide an additional 10-100 TW (Clarke et al. 1987). However, a considerable part of this energy input may be deposited below the homopause, from where much of the UV auroral radiation emanates. Below the homopause, hydrocarbons radiate away the energy very efficiently (Drossart et al. 1993), meaning that the actual direct energy input into the upper atmosphere is probably less than $10 \mathrm{TW}$ globally (Miller et al. 2005). Thermally driven winds could distribute the large amounts of energy deposited in the auroral regions (Waite et al. 1983) however an "energy gap" is expected due to the rapid planet rotation. Müller-Wodarg et al. (2012) have shown that a global energy input to the lower thermosphere of Saturn can increase the exospheric temperature from the $\approx 180 \mathrm{~K}$ predicted from solar heating alone to $\approx 410 \mathrm{~K}$, in close agreement with the measurements of Smith et al. (1983). Clarke et al. (2009) showed that there is a correlation of the Jovian auroral brightness with the solar wind pressure and Nichols et al. (2009a) concluded that enhancements of the main auroral emissions and partially the polar emissions are associated with solar wind compression regions. Moreover, Gurnett et al. (2002) discovered that there is solar wind modulation of Jupiter's radio emission. In addition, recent auroral observations of an Earth-like transpolar arc at Saturn (Fig. 7) (Radioti et al. 2014) may be related to solar wind driven tail reconnection similarly to the terrestrial case (Milan et al. 2005). Based on these findings it becomes clear that the role of space weather in driving energy deposition in the giant planets upper atmospheres could be important.

Before hitting the atmosphere, charged particles also emit radio waves into space. In a recent study, Nichols et al. (2012) suggested that auroras do occur also on bodies outside our Solar System and the auroral radio emissions are powerful enough to be detectable across interstellar distances. Such a result indeed indicates that planetary space weather could be far more intense in environments other than our Solar System hence understanding its features and processes could also be of help when investigating the characteristics of other Solar Systems as well as the exoplanet physics. Recently, Hallinan et al. (2015) reported simultaneous radio and optical spectroscopic observations (obtained with the Karl G. Jansky Very Large Array (VLA) radio telescope and the Double Spectrograph (DBSP) on the 5.1-m Hale telescope, respectively) of auroral emissions of an object at the end of the stellar main sequence (i.e. at the boundary between stars and brown dwarfs). Hallinan et al. (2015) argued that these aurorae are powered by processes originating much further out in the magnetosphere of the dwarf star (with respect to the heliosphere). Although the nature of the electromagnetic engine powering brown-dwarf aurorae is still to be determined, the scenario of the subcorotation of magnetospheric plasma on closed field lines, powering in turn magnetosphere-ionosphere coupling currents, has been suggested as a plausible model for this case (Schrijver 2009; Nichols et al. 2012). The mass loading required in this model can be achieved through interaction with the interstellar medium (ISM), the sputtering of the dwarf atmosphere by auroral currents, a volcanically active orbiting planet or magnetic reconnection in the photosphere. Moreover, Hallinan et al. (2015) showed that since the observed auroral emissions depended mainly on magnetic dipole moment and rotation (and may have been weakly coupled to other physical characteristics), aurora may be present at detectable levels even in the faintest $\mathrm{T}$ and $\mathrm{Y}$ dwarfs and bodies as well as from exoplanets.

\subsubsection{The role of galactic cosmic rays and transient events}

The modulation of the GCR by the disturbed solar wind (e.g. CMEs) in the near-Earth space has been studied for several solar cycles both in theoretical basis and through observations 
C. Plainaki et al.: Scientific aspects and future perspectives of planetary space weather

Table 4. Comparison of predicted and measured exospheric temperatures, Texo, of the giant planets. From Miller et al. (2005).

\begin{tabular}{lcccc}
\hline \hline Solar system giant planet & Heliocentric distance (AU) & Absorbed solar flux $\left(\mathrm{W} \mathrm{m}^{-2}\right)$ & $T_{\text {exo }}$ observed (K) & $T_{\text {exo }}$ calculated (K) \\
\hline Neptune & 30.07 & $1.1 \times 10^{-6}$ & 600 & 132 \\
Uranus & 19.19 & $2.7 \times 10^{-6}$ & 800 & 138 \\
Saturn & 9.57 & $1.1 \times 10^{-5}$ & 420 & 177 \\
Jupiter & 5.20 & $3.7 \times 10^{-5}$ & 940 & 203 \\
\hline
\end{tabular}

(Dorman 2009). The interaction of GCR with the terrestrial atmosphere results in the formation of atmospheric cascades and the generation of secondary species with both the spatial and energy distributions determined by the incident GCR spectrum and the atmosphere itself. In a similar way, the GCRs entering the Venusian atmosphere have been shown to generate a series of secondary neutral and ionized species being the major ionization source in the Venusian middle and lower atmosphere (below $\sim 100 \mathrm{~km}$ ) and related possibly with the cloud formation process in this planet (Nordheim et al. 2015). Recently, UV and VIS observations of Titan's emissions during its 2009 eclipse by Saturn revealed a weak airglow around the moon, as well as a brighter emission from its disk (West et al. 2012). In the absence of solar XUV stimulus, the role of the precipitating cosmic rays in producing Titan's airglow and chemistry could be estimated. Although cosmic ray ionization and chemiluminescence were the proposed candidates for these emissions (West et al. 2012), Lavvas et al. (2014) showed that the observed limb emissions were consistent with magnetospheric plasma energy input, while emissions instigated by cosmic ray excitation were too weak to explain the observed disk emissions. These authors concluded that in order to explain the observed disk emissions, the scattered stellar light from Titan's disk is the most likely scenario. It is worth mentioning, however, that according to Gronoff et al. (2011), the ionization by each Z-group of cosmic rays in the atmosphere of Titan results in the formation of an ionization layer peaking at $65 \mathrm{~km}$ altitude, independently of the solar activity, in consistency with the Huygens measurements (Hamelin et al. 2007; López-Moreno et al. 2008). The observed correlation between cosmic ray ionization altitudes and aerosol layers urges for further investigations in this direction in the future.

Transient events such as micrometeoroid dust particle impacts can be considered as potential external suppliers of water into all other outer planet stratospheres, modifying locally and temporarily the space weather conditions at these environments. The similar $\mathrm{H}_{2} \mathrm{O}$ fluxes per unit area found in the four giant planets and Titan (Feuchtgruber et al. 1997; Coustenis et al. 1998; Moses et al. 2000), combined with the rather constant dust flux $\left(\sim 3 \times 10^{-18} \mathrm{~g} \mathrm{~cm}^{-2} \mathrm{~s}^{-1}\right)$ measured in interplanetary space beyond $5 \mathrm{AU}$ (Landgraf et al. 2002), have been regarded as evidence that micrometeoroids interplanetary dust particles (IDPs) or interstellar - are the dominant source (Moses et al. 2000). Hartogh et al. (2011) showed that Enceladus is a quantitatively viable source of Saturn's water, evidencing the role of local or sporadic sources in the dynamic interactions between planetary atmospheres and particles.

\subsubsection{Space weather and interstellar medium}

Planetary space weather is also affected by the interstellar medium, which starts to affect the solar wind well before the Termination Shock (TS), the region of the heliosphere where the solar wind slows down to subsonic speed. The neutrals in the Local Interstellar Medium (LISM) can enter the heliosphere reaching unchanged the various planetary environments of the Solar System. The helium reaches Earth essentially unchanged from the LISM except for the acceleration by the Sun's gravity, whereas the LISM H is coupled to the protons in the heliosheath via charge exchange and thus is slowed, heated and diverted in the flow direction compared to the He (Richardson 2010). These LISM neutrals are ionized, the He inside $1 \mathrm{AU}$ and the $\mathrm{H}$ outside $5 \mathrm{AU}$. The generated pick-up ions are accelerated up to the solar wind speed and have an initial temperature equal to the solar wind energy, i.e. $\sim 1 \mathrm{keV}$. They are the dominant heliospheric components outside $\sim 10 \mathrm{AU}$ and by the TS almost the $20 \%$ of the total ion population consists of pick-up ions (Richardson 2010). Since the latter are hot, they influence the dynamics and composition of the solar wind hence its interactions with planetary environments, especially beyond the distance of $\sim 10$ AU from the Sun. Therefore, the pick-up ions are an important component of space weather at these locations. In this context, knowledge of the overall structure of the heliosphere is necessary. ENA images obtained by the Ion and Neutral Camera (INCA) on-board Cassini (Krimigis et al. 2009) revealed a broad belt of energetic protons with non-thermal pressure comparable to that of the local interstellar magnetic field. Such a picture for the shape of the heliosphere was not consistent with the previously believed cometary-type interaction of the possible bow shock and/or heliopause, heliosheath and TS (Fichtner et al. 2006). Energetic neutral atom cameras on board the Interstellar Boundary Explorer (IBEX) mission of NASA mapped the heliospheric boundary at lower $(\sim 6 \mathrm{keV})$ energies (Funsten et al. 2009; McComas et al. 2009). Both IBEX and INCA identified in the energetic neutral atom images dominant topological features (ribbon or belt) that can be explained on the basis of a model that considers an energetic neutral atominferred non-thermal proton pressure filling the heliosheath from the termination shock to the heliopause (Krimigis et al. 2009). Such results should be considered in future models devoted to planetary space weather purposes, especially when it concerns the outer Solar System science.

\subsection{Space weather and upcoming Solar System missions: current necessities and future science investigations}

Global radiation modelling including parameterization of space weather conditions in theoretical or empirical plasmainteractions models would be a very useful tool to support future in-situ observations during space missions. Exosphere and atmosphere modelling linked to the space weather variability around planetary bodies, determined either by the Sun or by strong parent planet magnetospheres, is absolutely necessary for the planning of related space observations and synergies between different mission-payload instruments and for the interpretation of space weather related effects, already 
registered by past missions (e.g. the atmospheric escape at Mars due to solar wind effects, or the aurora variability at Jupiter, related to the solar wind activity).

Numerous missions up to the orbit of Mars have provided a relatively comprehensive view of space weather conditions in the inner Solar System (except perhaps for Mercury's environment, for which the BEPI COLOMBO mission is expected to provide important add-ons to the current MESSENGER results). In addition, the ongoing MAVEN mission is continuously providing new important material related to space weather at the red planet. On the other hand, there are many uncertainties about physical processes revealed in the space surrounding the outer planets and their moons. In view of future missions to the outer Solar System, an accurate identification of the space weather conditions around a planet can contribute significantly in the estimation of the water abundances in the planetary atmospheres and their long- and short-term variability. Such an issue can play a significant role in identifying possible conditions for habitability, one of the main targets of the upcoming ESA JUICE mission. Recently, in the context of the study of habitability conditions in our Solar System, the payload for a future NASA mission to Europa has been selected. The determination of the vertical profile of water in the atmospheres of the outer planets and Titan is a diagnostic of its origin (e.g. cometary impact vs. permanent dust deposition), but also of the rate at which water is transported within and removed from the atmosphere (Hartogh et al. 2009). On the basis of this rate, it will be possible to estimate accurately the key input fluxes for discriminating between possible sources. In this context, studies performed for different space weather conditions are really important. Timescales for relevant observations should be determined on the basis of the variability timescales for the critical parameters, such as the vertical and horizontal mixing times of water in the planetary atmospheres. The NASA JUNO mission, arriving at Jupiter in August 2016, is dedicated to the study of Jupiter's atmosphere, ionosphere and high-latitude magnetosphere thus providing important data for the characterization of the space weather around the giant planet. In particular, with JUNO it will be possible to characterize plasma sources and losses through a series of in-situ measurements by different instruments on-board spacecraft (Bagenal et al. 2014).

In view of future missions to Solar System bodies, understanding the particle environments and their interactions with space radiation becomes crucial. For example, the presence of high fluxes of charged energetic particles in the radiation belts of a planet can affect electronic systems on-board spacecraft, endangering their normal operation. Planetary space weather forecasting is essential in order to design risk mitigation architectures that will ensure the undamaged operation of electronic systems aboard a spacecraft under different environment conditions and, eventually, guarantee the safety of astronauts during manned missions. Such architectures are expected to include radiation monitoring, effective spacecraft shelter and reliable communication systems. Eventual real-time monitoring of the space environment can contribute substantially in this direction.

The role of atmospheric drag in satellite aero-entry/ aerobraking (Duvall et al. 2005; Forbes et al. 2006) is strongly dependent on planetary space weather conditions. Specification of the neutral atmosphere density, its scale height and its variability, as driven by the solar spectral irradiance in the UV, by Joule heating by ionospheric currents and/or by an intense radiation environment (e.g. in case of bodies embedded in giant magnetospheres) all depend on planetary space weather conditions. Engineering issues related to the drag effect in Titan's atmosphere are not yet fully understood. Continuous monitoring of the EUV flux is required to mitigate disruptions in satellite communication, and in remote sensing.

In an overall effort to consider planetary space weather effects during future mission planning, solar monitoring is essential. Especially current and future missions in the inner Solar System (e.g. BEPI COLOMBO), having operations that are highly affected by space weather, could be supported by a series of solar observations. The lack of continuous observations could be compensated by the outputs of global MHD models. In summary, the main directions of the ongoing efforts in the planetary space weather discipline are:

1. Radiation modelling including integration of the supporting measurements at different planetary environments such as Mercury, Venus, Mars, Jupiter and Saturn;

2. Observations of solar wind and interplanetary medium conditions in a wide range of heliospheric distances, and development of tools to model CME, CIR and SEP propagation through the Solar System (e.g. the CDPP propagation tool: http://propagationtool.cdpp.eu/);

3. Modelling of planetary and satellite atmospheres and exospheres; modelling of the coupling processes between these environments and the parent planets' magnetospheres;

4. Investigation of ionospheric effects on radio signals;

5. Solar (UV, X-rays) monitoring.

We note that the absence of continuous solar spectral irradiance measurements has favoured the use of different solar proxies, such as the solar radio flux at $10.7 \mathrm{~cm}$ (F10.7 index), the sunspot number and the MgII core-to-wing index (Lilensten et al. 2014). However, according to Dudok de Wit et al. (2009) a single proxy cannot reproduce the variability in a spectral band at all timescales and, moreover, significant discrepancies in solar EUV-proxy correlations on solar cycle and solar rotation timescales can appear.

Whereas knowledge of space weather conditions at different planetary systems is a requisite for future mission planning, the scientific data to be obtained by these missions are going to provide in turn an important feedback for interdisciplinary science in the field of planetary space weather. Below we report two indicative examples, related to the future ESA JUICE and ESA/JAXA BEPI COLOMBO missions, in the outer and inner Solar System, respectively.

\subsubsection{Outer Solar System: space weather science with JUICE}

One of the highest priorities for future space missions is the Jupiter system and, specifically, its moons Europa and Ganymede. The ESA JUICE L1 mission will mainly characterize Ganymede and its magnetosphere, the Jovian magnetosphere and its dynamics, and it will provide information on the environments of Europa and Callisto (Krupp 2015). A deep knowledge of the radiation environment at Jupiter is of high importance particularly for determining critical parameters for the various JUICE payload instruments during different observational phases of the mission. All payload instruments of the JUICE mission have to be tested to survive damage from the radiation belts once they are located in the vicinity of the giant planet. In this context, identification of the planetary 
space weather conditions in the Jupiter system, through modelling and past data analysis, becomes of crucial importance.

In the Jupiter system, the identification of the chemical and physical characteristics of the subsurface ocean of the large moons is one of the essential objectives of the JUICE mission. A possible detection of the liquid water oceans in the moons' interior could be achieved thanks to the detection of induced magnetic fields originating from the interaction of the magnetospheric ions with these oceans (Khurana et al. 2009). Plasma currents above the moon surfaces create a major background signal during the detection of intrinsic oceanic signals (Sittler et al. 2013) hence the identification of their variability, as determined by in-situ plasma current measurements and associated models, is significant for setting the limits on the ocean source of the induced magnetic field. In addition, the measurement of the composition of the ejected - through sputtering and radiolysis - material provides the necessary feedback for distinguishing the abundances in the magnetospheric ion composition originating from the surface, from those originating from the ocean (Sittler et al. 2013). In this context, monitoring of the space weather conditions around the icy moons offers a unique possibility to complement the subsurface ocean measurements and to possibly assess their variability. Of particular importance for the monitoring of space weather conditions at Jupiter's icy moons is the determination of the currents due to ionospheric and pick-up ions, e.g. $\mathrm{O}_{2}{ }^{+}$in the case of Europa.

Detection of high-energy neutral atoms (HENA) and low-energy neutral atoms (LENA) at the Jovian system, with the Jovian Energetic Neutrals and Ions (JENI) and the Jovian Neutral Atoms (JNA) sensors, respectively, on-board JUICE/ PEP, in the energy range $0.01-300 \mathrm{keV}$, will provide information regarding the characteristics of the Io plasma torus and the radiation environment, as well as the related acceleration mechanisms (Futaana et al. 2015). The simultaneous imaging of the Io plasma torus at different mission phases will therefore permit the investigation of variations in plasma energy and spatial density, providing space weather monitoring in a short timeframe within the JUICE mission. Moreover, investigation of the trans-Europa gas torus (Mauk et al. 2003) through LENA measurements could provide complementary information on the interactions between Jupiter's magnetospheric plasma and the moon's exosphere. Using both energy spectra and their periodic features, together with the dispersion signatures and the mass spectra, it will be possible to distinguish between the Io and Europa tori and hence to determine the actual space weather conditions at the point of the measurement. We note that the energy spectrum of LENAs from Io should have a peak around $\sim 74 \mathrm{~km} / \mathrm{s}$, whereas the LENAs from Europa should have a peak around $\sim 150 \mathrm{~km} / \mathrm{s}$, corresponding to their respective corotation velocities (Futaana et al. 2015). Additionally, through the UV, plasma and LENA measurements with JUICE, it will be possible to search for spatial and temporal variations in the neutral and plasma densities at the satellites' near-surface regions.

\subsubsection{Inner Solar System: space weather science with BEPI COLOMBO}

The exploration of Mercury is expected to provide a major feedback for interdisciplinary science in the context of planetary space weather. After the first in-situ measurements provided by three flybys of Mariner 10 (Vilas et al. 1988) in 1974-1975, as described in Section 2.2.2, our knowledge of the Mercury environment has been greatly improved by the recent NASA MESSENGER mission. Based on the scientific return of this mission, it has been possible to address many issues related to the environment of Mercury, nevertheless, new questions have arisen. For example, it is still not known if the planet's magnetic field is shifted northwards. No clear evidence of hemisphere asymmetries was found in the $\mathrm{Na}$ exosphere distributions, which, on the other hand, seem to be related to the ion precipitation through the cusps. Whereas the precipitation of solar wind protons and upwelling surface ions has been observed in-situ by MESSENGER, the causeeffect relationship between these populations and the quickly accelerated ions of planetary origin has not been identified yet. Moreover, the electron and ion acceleration mechanisms are not yet completely understood. The role of planetary ions in the magnetosphere dynamics and in releasing new material after impacting the surface is still an open question. We also note that although the global exospheric intensity measured in-situ seems to be reproduced seasonally, the Na ground-based observations reveal distributions varying in hour timescales. Additionally, the exact mechanisms of the species-dependent generation process, their variability and the related spatial distributions have not yet been explained adequately. For example $\mathrm{Na}$ is released mainly on the dayside, while the $\mathrm{Ca}$ and/or Mg distributions seem to be unrelated to the planet's illumination. In fact, we would expect a major PSD effect on the dayside for volatile materials and a release of both volatiles and refractories, due to ion precipitation and micrometeoroid impact vaporization. If the current system closes onto the surface, given the absence of an ionosphere, what is the surface conductivity? Which is the role of induction effects, as a response to Sun's activity? In fact, the complex Hermean system requires in-depth investigations to explain the major in-situ and ground-based observational evidences and to draw a coherent global picture of the system's functioning.

The tools for the investigations related to space weather at Mercury are on one hand the development of more accurate magnetospheric and plasma circulation theoretical models (including induction effects, high reconnection rates, fast variability and effects of the surface and exosphere interaction) and on the other hand, the development of exosphere multiprocesses generation models including the effects of the environment's interaction with the Sun's radiation. The modelling efforts should be paired up with further groundbased observations and, later, with the observations of the Mercury-dedicated ESA/JAXA mission, BEPI COLOMBO (Benkhoff et al. 2010). The BEPI COLOMBO mission consists of two spacecraft orbiting at Mercury: the Mercury Magnetospheric Orbiter (MMO), with its spinning eccentric orbit, is fully dedicated to magnetosphere and Hermean environment topics while the Mercury Planetary Orbiter (MPO), with its low altitude orbit, is mainly dedicated to the planet's observation and to the monitoring of its response to external agents. It will be the first time that coordinated multi-point observations could be obtained in a planet different from the Earth (Milillo et al. 2010). Joint observations from the two spacecraft are of key importance because many phenomena in Mercury's environment are temporarily and spatially varying.

A deep analysis of the environment phenomena at Mercury could be of great importance during the BEPI COLOMBO science operation planning. In fact, some gaps in the MESSENGER results are due to the limitation in spatial coverage, due to the highly eccentric orbit with periherm close 
to the Northern hemisphere and/or to limitation in observations coverage. For example, the $\mathrm{Na}$ observations were limited to equatorial regions (Cassidy et al. 2015), therefore, a possible interpretation of mismatch with the ground-based observations results could have been obtained. BEPI COLOMBO, with the MPO/SERENA particle package (Orsini et al. 2010), will observe in the dayside simultaneously the precipitating ions (by SERENA/MIPA) and the backscattered ENA from the surface (by SERENA ELENA) as well as the bulk exosphere composition (by SERENA/STROFIO). The MPO/MAG magnetometer will complement the in-situ measurement, while the MMO could be in the favourable condition to monitor the upstream solar wind and IMF. This full monitoring will permit to have a comprehensive picture of the cause-effect link and to finally reveal the still unsolved problem of the surface release mechanisms (Milillo et al. 2010). This is just an example of the deep investigation of Sun-Mercury interaction that will be obtained with this outstanding mission.

The comprehensive payload of the BEPI COLOMBO mission includes, besides the fields and particles sensors, also a radiation monitor (BERM), and a Sun X-Rays and SEP monitor (SIXS), which will both allow to have a monitoring service of the space weather conditions, possibly critical at Mercury's orbit. Since the Hermean magnetosphere occupies a unique position in the space plasma physics scenarios, the study of Mercury's magnetospheric processes will not only provide a clear picture of the planetary magnetosphere itself but it will also broaden our field of view of space plasma physics, in general.

\section{Planetary space weather as a tool to understand the circum-terrestrial space weather}

The lessons learned from the study of the interactions of planetary bodies with plasma and solar photon radiation can be an important feedback for further and more in-depth understanding of the circum-terrestrial space weather phenomena. Actually, there are several open questions related to this field; a comparative investigation among the results obtained through observations and/or modelling of different environments in the Solar System could provide important feedback for the interpretation of space weather phenomena at the Earth. We will briefly show how this can indeed take place, by focusing on some specific open issues in space weather science.

The terrestrial magnetopause standoff distance is known to be determined from the balance between the outer solar wind dynamic pressure and the inner magnetosphere pressure, which is generated by both the terrestrial dipole field and the fields generated by the magnetospheric current systems. For a fixed solar wind dynamic pressure the subsolar magnetopause is displaced toward the Earth when the IMF turns southward (Pudovkin et al. 1998), an effect that has been associated with the erosion caused by the magnetic reconnection (see also Sect. 3.1) at the dayside magnetopause (Samsonov et al. 2012). The opposite effect, namely, displacement of the subsolar magnetopause away from the Earth for a radial IMF orientation has also been observed (Merka et al. 2007) and studied in detail (e.g. Dušík et al. 2010). Despite the numerous studies on the position of the outer boundary of the terrestrial magnetosphere, in a recent paper Samsonov et al. (2012) showed that the total pressure on the magnetopause differs from the solar wind dynamic pressure in the majority of the examined cases. Such a result is also consistent with the expansion of the magnetosphere observed experimentally for an IMF radial direction, when no magnetic barrier is formed. In the isotropic MHD approximation, Samsonov et al. (2012) showed that the difference between the total pressure on the subsolar magnetopause during northern and radial IMFs does not exceed $10 \%-12 \%$, whereas in the anisotropic approximation this difference increases up to $15 \%-20 \%$. Nevertheless, as these authors also state, some cases of more substantial magnetopause displacements have been reported in the past (several radii of the Earth). Transition processes, such as oscillations of the magnetopause (e.g. Laakso et al. 1998) or kinetic effects, could be responsible for the magnetopause oscillation. Consequently their inclusion in the MHD models could lead to better approximations of the evolutionary changes of the terrestrial magnetosphere. From the above it is clear that an improvement of the current models for the circum-terrestrial space weather and an in-depth comparison with the available data are necessary in the future. In such a context, MHD simulations destined for the solar wind interactions with the magnetospheres of planetary bodies other than the Earth could be of great help since they provide an extended range of variability for the various factors determining space weather in the near-body environment. In particular, considering the evolution of the solar wind from 0 to $5 \mathrm{AU}$ (see study by Hanlon et al. 2004), a more complete set of solar wind parameter values can be obtained and further used for constraining the MHD models applied for the Earth case.

In the context of the circum-terrestrial space weather, a phenomenon of particular interest is the magnetospheric substorm, also referred to as auroral substorm or, simply, substorm. A magnetospheric substorm is a transient process initiated on the nightside of the Earth in which a significant amount of energy derived from the solar wind-magnetosphere interaction is deposited in the auroral ionosphere and in the magnetosphere (Rostoker et al. 1980). The process consists of the extraction of energy from the solar wind, the storage of this energy in the magnetotail leading to an increase of the lobe magnetic flux, the formation of a thin current sheet within the tail plasma sheet, the disruption of the tail current and the release of a plasmoid (Baker et al. 1999). From the observational point of view, a substorm, contrary to a geomagnetic storm, is seen primarily at the polar regions as a sudden brightening and increased movement of the auroral arcs, taking place over a period of a few hours. Although, the progress in studying auroral substorms at Earth (consisting of the growth phase, the expansion phase and the recovery phase) has been substantial in the last 50 years, there are still several details in the related physical mechanisms that have not been identified. In particular, according to Akasofu (2015), it is still not understood how could the unloaded energy produce a secondary dynamo, which powers the expansion phase of the magnetospheric substorm. Some information that may be of help for answering such open questions in a coherent frame can be obtained also through the study of equivalent phenomena in other planetary systems (where existent). For example, Mercury has a high reconnection rate in the dayside with a strong coupling between the solar wind and the magnetosphere and there is evidence of nighside reconnection (Raines et al. 2014). Moreover, there is increasing evidence that internally driven nightside (or tail) reconnection processes occur also in the Jovian magnetosphere (Nishida 1983; Louarn et al. 1998; Woch et al. 1998, 1999; Russell et al. 2000). To describe these processes, Kronberg et al. (2005) have used the term "substorm-like", whereas in other works the phenomenon is 
also referred to as "Vasyliunas tail reconnection" process. Although the overall morphology of an internally driven nightside reconnection process in the Jovian magnetosphere appears at first sight very much similar to the terrestrial one (Kronberg et al. 2005), the observed disturbances at Jupiter seem to re-occur with a much longer characteristic time constant of 2-4 days (Louarn et al. 1998; Woch et al. 1998; Radioti et al. 2008b). This intrinsic periodicity has led to the suggestion that nightside reconnection processes in the Jovian system are not driven by solar wind-magnetosphere interactions but represent an internally-driven process (Kronberg et al. 2005). More specifically, at Jupiter, it is believed to be the centrifugal force on rapidly rotating, massloaded flux tubes leading to a thinning of the plasma sheet, which enables spontaneous reconnection (Vasyliunas 1983). Russell et al. (2000) analysed Galileo data of the Jovian current sheet and found that on the nightside of the magnetodisk beyond $50 R_{\mathrm{J}}$ the strength of the perturbation in the normal component increases so that periodically (but approximately once every $4 \mathrm{~h}$ ) the normal component rises above $2 \mathrm{nT}$, either northward or southward, and once a day, above $5 \mathrm{nT}$. These enhancements were attributed to episodic reconnection of confined patches of the near-Jovian magnetotail and clearly indicated that fast rotation of the Jovian magnetosphere produces important differences between Earth's magnetotail and Jupiter's magnetodisk. Nevertheless, the phenomenon is not yet completely understood as Vogt et al. (2010) showed that the origin of the nightside reconnection at Jupiter might not be only internal; there is also evidence of solar wind driven tail reconnection. On the basis of these findings and the results of other studies referring either to the Jovian system (e.g. Southwood \& Kivelson 2001; Woch et al. 2002) or to the Saturnian one (e.g. Cowley et al. 2005; Jackman et al. 2009), it is clear that investigation on tail reconnection physics at magnetospheric systems other than the Earth's can provide important feedback for a better understanding of the substorm physical mechanisms at our own planet.

The study of planetary atmospheres other than the Earth's, and in particular the study of the role of plasma-neutral interactions in their evolution, could contribute to our understanding of the long-term space weather (referred to also as space climate) effects and finally the origins of life itself (Yamauchi \& Wahlund 2007). Titan is particularly interesting in this respect, as its atmosphere is thought to resemble the prebiotic Earth (Molina-Cuberos et al. 2001).

In the outer Solar System the interactions between solar wind and planetary environment can be significantly influenced by the interstellar pick-up ions hence a detailed evaluation of the role of the latter at large distances from the Sun could be of help in understanding better such processes in the near-Earth space. In particular, the measurements of the interplanetary magnetic field fluctuations together with those of plasma and pick-up ion density variations, at different distances from the Sun, can provide information on the origin of turbulence in the solar wind and its evolution from its source to the heliopause. Comparative planetology in the context of planetary space weather will therefore allow to set constraints on the processes of energy transfer in different regions of the Solar System and hence to understand the mechanisms of the energy dissipation also in the circum-terrestrial space.

As an example of how results obtained through studies in planetary magnetosphere physics are essential for our understanding of terrestrial phenomena related to space weather, we point to the study of the ring current at Saturn by Krimigis et al. (2007). These authors analysed images of the ring current at Saturn based on measurements from the magnetospheric imaging instrument (MIMI) on-board Cassini and found that the ring current can be highly variable with strong longitudinal asymmetries that corotate nearly rigidly with the planet. This result by Krimigis et al. (2007) contrasts with the Earth's ring current, where there is no rotational modulation and initial asymmetries are organized by local time effects. A comparative view, therefore, of the same phenomenon at two different environments within the Solar System can be essential for our in-depth understanding of the physical mechanisms which govern it.

\section{Summary and concluding remarks}

In this article, we attempted to present the current state of planetary space weather and to analyse future necessities. We first discussed the definition of planetary space weather and showed its in-depth relation to the interactions between environments and fields. We then examined its special characteristics at different regions of the Solar System, analysing the effects of solar wind, solar energetic particles, UV flux, cosmic rays, and magnetospheric populations, on planetary and lunar atmospheres.

Starting with the outer Solar System, we discussed the effect of a strongly tilted non-axial magnetic field in the case of Uranus and Neptune, leading to a series of open questions, including the one on the nature of the interaction of the magnetospheres with the moons. In the case of the Saturn system, our understanding on planetary space weather has been essentially improved thanks to extended observations of the Cassini mission. However, the interactions of Saturn's giant magnetosphere (and the associated currents) with the moons have not been yet fully investigated. Titan constitutes of course a major goal as its $\mathrm{N}_{2} / \mathrm{CH}_{4}$ atmosphere is dense hence subject to a series of short- and long-term interactions with the magnetospheric plasma and the UV environment. Moreover, Enceladus, a major source of water-ions and ionized waterproducts for the Saturn system, is expected to be a body of major interest in the context of planetary space weather. Approaching the Sun, Jupiter introduces a new step in the complexity, as it adds the interaction between the planet's magnetosphere and a magnetized body (Ganymede) within the same planetary system. Ganymede, the only known satellite possessing an intrinsic magnetic field, is strongly subject to space weather since variations in the plasma environment in its vicinity result in different morphologies of its sputter-induced exosphere. The radiolytically produced $\mathrm{O}_{2}$ exosphere, as at Europa, is expected to depend not only on the plasma impacting the surface but also on the moon's illumination by the Sun (Plainaki et al. 2013, 2015). Therefore, the overall result of variable plasma conditions at the vicinity of these moons will be the effect of both space weather and the body's orbital motion. As a consequence, the Galilean moons along their orbit around Jupiter are expected to have exospheres with different morphologies which in any case get dynamically configured by the plasma source and loss processes (see for example Plainaki et al. 2013; Sittler et al. 2013). Some of the most intriguing open questions related to space weather in the outer Solar System are the following:

- How does the solar wind determine the strength and morphology of auroral features in the giant planets? 
- What is the dependence of the atmospheric energy balance at giant planets on solar wind-magnetosphere coupling?

- What is the major plasma source at Neptune and which are the factors determining its variability? Are magnetospheric plasma motions at the distance of $\sim 30 \mathrm{AU}$ dominated by solar wind or planetary rotation?

- What are the global configurations of the magnetospheres of Uranus and Neptune and how do they determine plasma dynamics during different seasons and periods of solar activity?

- How do the plasma-neutral interactions at Titan compare with those at the Galilean satellites?

- How is space weather related to plasma-surface interactions at Ganymede?

- What is the role of internal exosphere sources (e.g. ice sputtering, gas plumes) in determining the actual space weather conditions around Europa?

The inner Solar System looks somehow more familiar. The atmospheric escape (existent also in the outer Solar System as well) is a major feature in need of a thorough exploration. Moreover, the inner Solar System provides a set of configurations (e.g. with or without a magnetic field, with or without an atmosphere), which are easier to observe from the Earth or from space. Mercury, the terrestrial planet that up to now has been investigated to a lesser extent, offers an excellent future possibility to study space weather in a region very close to the body's parent-star. Extreme conditions of solar wind, UV flux and surface temperature, in combination with patterns of plasma precipitation to the surface guided by Mercury's magnetic field, result in a dynamic exosphere configuration. Solar events such as CMEs and/or SEP events activate macroscopic planetary space weather phenomena; in fact, they have a direct and strong impact on Mercury's environment inducing planet reaction generating surface currents, induction effects, reconnection and flux transfer events, with variability timescales much shorter than the ones at the Earth's case. In this context, the future BepiColombo mission with two spacecraft and with its comprehensive payload, and in particular with the SERENA experiment on board the MPO spacecraft, will provide important feedback for characterizing space weather in Mercury's vicinity. Moreover, the study of the relation between the environments of Mercury and Venus with the Sun could be useful also for the interpretation of the observations of exoplanets, which are often located at close distances to their parent-star, hence in conditions similar to the ones at these two terrestrial planets. Some outstanding open questions related to space weather in the inner Solar System are the following:

- What is the cause-effect relationship between solar wind, surface-released particles and ions of planetary origin quickly accelerated at Mercury?

- If the current system in Mercury's magnetosphere closes onto the surface, given the absence of an ionosphere, which is the surface conductivity? Which is the role of induction as a response to solar activity?

- What is the role of GCR and SEPs in Venus' atmospheric chemistry and cloud formation during different conditions of solar activity?

In the current paper, we also discussed how feedback for answering several open questions related to planetary space weather can be obtained from the future ESA JUICE and
ESA/JAXA BEPI COLOMBO missions. As a conclusion, we would like to list some of the possible scientific objectives of hypothetical future missions related to space weather:

In the outer Solar System

- to determine the role of solar wind in auroral features in planetary and lunar atmospheres;

- to determine the role of solar wind in configuring the planetary magnetospheres, especially at large distances from the Sun and to understand the atmospheric energy balance and magnetosphere coupling at the giant planets;

- to understand plasma dynamics at different planetary systems during different seasons and periods of solar activity and to determine plasma sources and losses and their variability at short and long term;

- to understand the role of internal exosphere sources (e.g. ice sputtering, gas plumes) in determining the actual space weather conditions at the giant planet satellites.

\section{In the inner Solar System}

- to determine the cause-effect relationship between solar wind, GCRs, SEPs and surface-released (or atmosphere escaping) particles at the terrestrial planets;

- to understand the role of GCR and SEPs in atmospheric chemistry and possibly in cloud formation;

- to understand the role of space weather in the evolution history of terrestrial planets.

The first goal related to the achievement of such objectives could be the parameterization of both a series of physical quantities (and of their variations) determining the range and the intensity of space weather conditions and a series of quantities describing the actual resulting space weather phenomena at the vicinity of the Solar System body. Physical quantities belonging to the first category could be related to solar activity (SEP, CMEs, solar wind) and of course to the physical characteristics of bodies and planetary systems (e.g. dimension, dipole magnetic field, distance from the Sun etc.). Instead, physical quantities of the second category could be the body's magnetosphere morphology, the plasma density, composition and energy spectrum at the body's vicinity, the efficiency of surface-released material (in case of bodies with an exosphere), and the efficiency of the escape (in case of bodies with an atmosphere). The parameterized quantities of both categories would help to provide a first-order grouping of the related phenomena and to obtain feedback for determining the cause-effect relationship in space weather.

A strategy for investigating space weather in the entire Solar System is to select a series of instruments to send to different planets and to different zones in the heliosphere, in particular out of the ecliptic plane, in order to measure the solar wind, IMF or electric field at different solar latitudes. Due to the diversity of the planetary environments (e.g. in terms of energy and intensity ranges for the expected ion and electron populations), the best-suited instrument performances should be considered during each mission. Moreover, the payload instruments of a space mission devoted to space weather investigations (e.g. ion sensors, spectrometers etc.) should be selected in the best-suited configuration. Due to possible budget constraints, the number of instruments on board a spacecraft dedicated to planetary space weather 
investigations needs to be carefully considered. An indicative payload could include the following instruments:

- ion and neutral mass spectrometer;

- ENA imager;

- energetic particle detector;

- plasma wave instrument;

- magnetometer and electric field detector;

- simple diodes to analyse some features of the solar radiation and of the planetary emissions or, alternatively, a UV spectrometer.

What is of crucial importance is to have the instruments both calibrated and cross-calibrated. Moreover, it would be useful to have these instruments operating at periods where other missions are ongoing, in order to increase science output and perform multi-spacecraft studies.

The most critical aspects for the success of a hypothetical space weather mission in the outer Solar System are the availability of radioisotope-powered energy sources and the achievement of a decent data transfer rate. Solar panels for energy generation have been proposed in the past but they may not be feasible or practical at long distances from the Sun (Turrini et al. 2014). Americium-based thermoelectric generators are already under study in Europe (Ambrosi 2013). On-board data compression, installation of more stations on the ground and calibration of the data volume to be collected during different mission phases with an acceptable downlink rate could provide solutions to the problem of efficient data transfer rates.

Acknowledgements. The authors would like to thank the referee for valuable comments that helped to improve the quality of the paper. This paper is financially supported by the Italian Space Agency (ASI) under contract "SERENA", No. I/090/06/0. The idea for writing this article came out after a series of discussions in the context of the 11th European Space Weather Week (2014). JL's contribution is under the European contract EUROPLANET Horizon 2020 research and innovation programme under Grant Agreement No. 654208, Task 4, package 7: VA1-PSWD (Planetary Space Weather and Diary) and under grant by the French Programme National de Planétologie. AR is supported by the PRODEX Programme managed by the European Space Agency in collaboration with the Belgian Federal Science Policy Office. Discussions in this paper have been partially performed in the context of the activities of the 2014 ISSI International Team \#322, Towards a global unified model of Europa's exosphere in view of the JUICE mission, http://www.issibern.ch/teams/exospherejuice/. Simulation results based on the WSA-ENLIL+Cone model have been provided by the Community Coordinated Modeling Center at Goddard Space Flight Center through their public Runs on Request system (http://ccmc.gsfc.nasa.gov; run number: Alexey_Isavnin_011316_SH_1). The WSA model was developed by N. Arge at AFRL and the ENLIL Model was developed by D. Odstrcil at GMU. The authors thank G. Gronoff (LARC - NASA) and David Pawlowski (East Michigan University) for their help in getting the data for the Mars upper atmosphere, Mathieu Barthelemy (Grenoble University Space Center, France) for helpful discussions concerning space weather applications, Sergio Fabiani (INFN, Italy) and Alda Rubini (INAF-IAPS, Italy) for discussions on technological issues regarding future space missions, and Leila Mays (NASA) for providing material based on the WSA-ENLIL+Cone simulations. The authors also thank Chris Arridge (Lancaster University, UK) and Fran Bagenal (University of Colorado, USA) for useful feedback considering the magnetosphere of Uranus, Alessandro Mura (INAF-IAPS, Italy) and Helen Mavromichalaki
(National and Kapodistrian University of Athens, Greece) for fruitful discussions on interplanetary physics and circum-terrestrial space weather, and Panayiotis Lavvas (CNRS, France) for providing feedback related to Titan atmospheric science. The editor thanks an anonymous referee for the assistance in evaluating this paper.

\section{References}

Abel, B., and R.M. Thorne. Relativistic charged particle precipitation into Jupiter's sub-auroral atmosphere. Icarus, 166, 311-319, 2003, DOI: 10.1016/j.icarus.2003.08.017.

Acuña, M.H., J.E.P. Connerney, N.F. Ness, R.P. Lin, D. Mitchell, et al. Global distribution of crustal magnetization discovered by the Mars global surveyor MAG/ER experiment. Science, 284 (5415), 790-793, 1999, DOI: 10.1126/science.284.5415.790.

Akasofu, S.I. Auroral substorms as an electrical discharge phenomenon. PEPS, 2, 20, 2015, DOI: 10.1186/s40645-015-0050-9.

Alexeev, I.I., E.S. Belenkaya, J.A. Slavin, H. Korth, B.J. Anderson, et al. Mercury's magnetospheric magnetic field after the first two MESSENGER flybys. Icarus, 209 (1), 23-39, 2010.

Ambrosi, R. European space nuclear power systems: enabing technology for space exploration missions, in: "Uranus beyond Voyager 2" Workshop, Meudon, France, 16-18 Sept., 2013.

Andersen, V. Observations of cosmic ray modulation with MARIE. $B A A S, \mathbf{1}, 38,2006$.

Anderson, B.J., C.L. Johnson, H. Korth, M.E. Purucker, R.M. Winslow, et al. The global magnetic field of Mercury from MESSENGER orbital observations. Science, 333, 1859-1862, 2011a.

Anderson, B.J., J.A. Slavin, H. Korth, S.A. Boardsen, T.H. Zurbuchen, J.M. Raines, G. Gloeckler, R.L. McNutt, and S.C. Solomon. The dayside magnetospheric boundary layer at Mercury. Planet. Space Sci., 59 (15), 2037-2050, 2011 b.

André, N., M. Blanc, S. Maurice, P. Schippers, E. Pallier, et al. Identification of Saturn's magnetospheric regions and associated plasma processes: synopsis of Cassini observations during orbit insertion. Rev. Geophys., 46, RG4008, 2008, DOI: $10.1029 / 2007 R G 000238$.

Andriopoulou, M., E. Roussos, N. Krupp, C. Paranicas, M. Thomsen, S. Krimigis, M.K. Dougherty, and K.-H. Glassmeier. Spatial and temporal dependence of the convective electric field in Saturn's inner magnetosphere. Icarus, 229, 57-70, 2014, DOI: 10.1016/j.icarus.2013.10.028.

Aplin, K.L. Atmospheric electrification in the Solar System. Surv. Geophys., 27, 63-108, 2006, DOI: 10.1007/s10712-005-0642-9.

Aplin, K.L. Electrifying atmospheres: charging, ionisation and lightning in the Solar System and beyond, in SpringerBriefs in Astronomy, Springer, Netherlands, Dordrecht, 2013, DOI: $10.1007 / 978-94-007-6633-4$.

Araki, T., S. Fujitani, M. Emoto, K. Yumoto, K. Shiokawa, et al. Anomalous sudden commencement on March 24, 1991. J. Geophys. Res., 102 (A7), 14075-14086, 1997, DOI: 10.1029/96JA03637.

Arge, C.N., and V.J. Pizzo. Improvement in the prediction of solar wind conditions using near-real time solar magnetic field updates. J. Geophys. Res. [Space Phys.], 105 (A5), 10465-10479, 2000.

Arge, C.N., J.G. Luhmann, D. Odstrcil, C.J. Schrijver, and Y. Li. Stream structure and coronal sources of the solar wind during the May 12th, 1997 CME. J. Atmos. Sol. Terr. Phys., 66 (15), 1295-1309, 2004.

Arridge, C.S., C.B. Agnor, N. André, K.H. Baines, L.N. Fletcher, et al. Uranus Pathfinder: exploring the origins and evolution of Ice Giant planets. Exp. Agric., 33, 753-791, 2012 ,

DOI: $10.1007 / \mathrm{s} 10686-011-9251-4$.

Arridge, C.S., N. Achilleos, J. Agarwal, C.B. Agnor, R. Ambrosi, et al. The science case for an orbital mission to Uranus: exploring the origins and evolution of ice giant planets. Planet. Space Sci., 104, 122-140, 2014, DOI: 10.1016/j.pss.2014.08.009. 
Badman, S.V., E.J. Bunce, J.T. Clarke, S.W.H. Cowley, J.-C. GeéRard, D. Grodent, and S.E. Milan. Open flux estimates in Saturn's magnetosphere during the January 2004 Cassini-HST campaign, and implications for reconnection rates. J. Geophys. Res. [Space Phys.], 110, A11216, 2005, DOI: $10.1029 / 2005 J A 011240$.

Badman, S.V., N. Achilleos, K.H. Baines, R.H. Brown, E.J. Bunce, M.K. Dougherty, H. Melin, J.D. Nichols, and T. Stallard. Location of Saturn's northern infrared aurora determined from Cassini VIMS images. Geophys. Res. Lett., 38, L03102, 2011, DOI: 10.1029/2010GL046193.

Badman, S.V., N. Achilleos, C.S. Arridge, K.H. Baines, R.H. Brown, et al. Cassini observations of ion and electron beams at Saturn and their relationship to infrared auroral arcs. J. Geophys. Res. [Space Phys.], 117, A01211, 2012, DOI: $10.1029 / 2011 \mathrm{JA} 017222$.

Badman, S.V., A. Masters, H. Hasegawa, M. Fujimoto, A. Radioti, D. Grodent, N. Sergis, M.K. Dougherty, and A.J. Coates. Bursty magnetic reconnection at Saturn's magnetopause. Geophys. Res. Lett., 40, 1027-1031, 2013, DOI: 10.1002/grl.50199.

Badman, S.V., C.M. Jackman, J.D. Nichols, J.T. Clarke, and J.-C. Gérard. Open flux in Saturn's magnetosphere. Icarus, 231, 137-145, 2014, DOI: 10.1016/j.icarus.2013.12.004.

Badman, S.V., G. Branduardi-Raymont, M. Galand, S.L.G. Hess, N. Krupp, L. Lamy, H. Melin, and C. Tao. Auroral processes at the Giant planets: energy deposition, emission mechanisms, morphology and spectra. Space Sci. Rev., 187, 99-179, 2015, DOI: 10.1007/s11214-014-0042-x.

Bagenal, F. Giant planet magnetospheres. Annu. Rev. Earth Planet. Sci., 20, 289-328, 1992.

Bagenal, F. Planetary Magnetospheres. In: T.D. Oswalt, L.M. French, and P. Kalas, Editors. Planets, Stars and Stellar Systems, Springer Science+Business Media, Dordrecht, 251, ISBN: 978-94-007-5605-2, 2013.

Bagenal, F., A. Adriani, F. Allegrini, S.J. Bolton, B. Bonfond, et al. Magnetospheric science objectives of the JUNO mission. Space Sci. Rev., 1-69, 2014, DOI: 10.1007/s11214-014-0036-8.

Bagenal, F., E. Sidrow, R.J. Wilson, T.A. Cassidy, V. Dols, F.J. Crary, A.J. Steffl, P.A. Delamere, W.S. Kurth, and W.R. Paterson. Plasma conditions at Europa's orbit. Icarus, 261, 1-13, 2015, DOI: 10.1016/j.icarus.2015.07.036.

Baker, D.N., T.I. Pulkkinen, J. Büchner, and A.J. Klimas. Substorms: a global instability of the magnetosphere-ionosphere system. J. Geophys. Res., 104, 14601-14612, 1999.

Baker, D.N., G. Poh, D. Odstrcil, N. Arge, M. Benna, et al. Solar wind forcing at Mercury: WSA-ENLIL model results. J. Geophys. Res. [Space Phys.], 118, 45-57, 2013, DOI: $10.1029 / 2012 J A 018064$.

Baker, V.R., R.G. Strom, J.M. Dohm, V.C. Gulick, J.S. Kargel, G. Komatsu, G.G. Ori, and J.W. Rice. Mars' Oceanus Borealis, ancient glaciers, and the MEGAOUTFLO hypothesis. Lunar and Planetary Institute Science Conference Abstracts, 31, 2000.

Ballester, G.E. Magnetospheric interactions in the major planets. In: W. Wamsteker, R. Gonzalez Riestra, and B. Harris, Editors. Ultraviolet Astrophysics Beyond the IUE Final Archive, 413, ESA Special Publication, 21, 1998.

Bampasidis, G., A. Coustenis, R.K. Achterberg, S. Vinatier, P. Lavvas, et al. Thermal and chemical structure variations in Titan's stratosphere during the Cassini mission. Astrophys. $J ., 760,144,2012$, DOI: $10.1088 / 0004-637 X / 760 / 2 / 144$.

Baron, R.L., T. Owen, J.E.P. Connerney, T. Satoh, and J. Harrington. Solar wind control of Jupiter's $\mathrm{H}^{+3}$ auroras. Icarus, 120, 437-442, 1996, DOI: 10.1006/icar.1996.0063.

Barth, C.A., C.W. Hord, A.I.F. Stewart, W.R. Pryor, K.E. Simmons, W.E. McClintock, J.M. Ajello, K.L. Naviaux, and J.J. Aiello. Galileo ultraviolet spectrometer observations of atomic hydrogen in the atmosphere of Ganymede. Geophys. Res. Lett., 24, 2147-2150, 1997.

Barthelemy, M., and G. Cessateur. Sensitivity of upper atmospheric emissions calculations to solar/stellar UV flux. J. Space
Weather Space Clim., 4 (27), A35, 2014, DOI: $10.1051 / \mathrm{swsc} / 2014033$.

Belcher, J.W., H.S. Bridge, B. Coppi, G.S. Gordon Jr., A.J. Lazarus, et al. Plasma observations near Neptune - initial results from Voyager 2. Science, 246, 1478-1483, 1989, DOI: $10.1126 /$ science. 246.4936 .1478 .

Belov, A., E. Eroshenko, H. Mavromichalaki, C. Plainaki, and V. Yanke. Solar cosmic rays during the extremely high ground level enhancement on 23 February 1956. Ann. Geophys., 23, 2281, 2005.

Benkhoff, J., J. van Casteren, H. Hayakawa, M. Fujimoto, H. Laakso, M. Novara, P. Ferri, H.R. Middleton, and R. Ziethe. BepiColombo-Comprehensive exploration of Mercury: mission overview and science goals. Planet. Space Sci., 58 (1-2), 2-20, 2010, DOI: 10.1016/j.pss.2009.09.020.

Berezhnoy, A.A., and B.A. Klumov. Impacts as a source of the atmosphere on Mercury. Icarus, 195, 511-522, 2008.

Bertaux, J.L., F. Leblanc, O. Witasse, E. Quemerais, J. Lilensten, et al. Discovery of an aurora on Mars. Nature, 435 (7043), 790-794, 2005.

Bertucci, C., D.C. Hamilton, W.S. Kurth, G.B. Hospodarsky, D.G. Mitchell, N.J.T. Edberg, N. Sergis, and M.K. Dougherty. Titan interaction with the supersonic solar wind: Cassini T96 observations. In: AGU Fall Meeting Abstracts, 4322, 2014.

Bertucci, C., D.C. Hamilton, W.S. Kurth, G. Hospodarsky, D. Mitchell, N. Sergis, N.J.T. Edberg, and M.K. Dougherty. Titan's interaction with the supersonic solar wind. Geophys. Res. Lett., 42, 193-200, 2015, DOI: 10.1002/2014GL062106.

Bishop, J., S.K. Atreya, P.N. Romani, G.S. Orton, B.R. Sandel, and R.V. Yelle. The middle and upper atmosphere of Neptune. In: D.P. Cruikshank, M.S. Matthews, and A.M. Schumann, Editors. Neptune and Triton, University of Arizona Pub., USA, 427-487, 1995.

Blake, J.B., H.H. Hilton, and S.H. Margolis. On the injection of cosmic ray secondaries into the inner Saturnian magnetosphere. I - Protons from the CRAND process, J. Geophys. Res. [Space Phys.], 88, 803-807, 1983, DOI: 10.1029/JA088iA02p00803.

Blanc, M., S. Bolton, J. Bradley, M. Burton, T.E. Cravens, et al. Magnetospheric and plasma science with Cassini-Huygens. Space Sci. Rev., 104, 253-346, 2002, DOI: $10.1023 / \mathrm{A}: 1023605110711$.

Blanc, M., D.J. Andrews, A.J. Coates, D.C. Hamilton, C.M. Jackman, et al. Saturn plasma sources and associated transport processes. Space Sci. Rev., 192 (1), 237-283, 2015, DOI: $10.1007 / \mathrm{s} 11214-015-0172-9$.

Bolton, S.J., S. Gulkis, M.J. Klein, I. de Pater, and T.J. Thompson. Correlation studies between solar wind parameters and the decimetric radio emission from Jupiter. J. Geophys. Res. [Space Phys.], 94, 121-128, 1989, DOI: 10.1029/JA094iA01p00121.

Bolton, S.J., M. Janssen, R. Thorne, S. Levin, M. Klein, et al. Ultrarelativistic electrons in Jupiter's radiation belts. Nature, 415, 987-991, 2002.

Bolton, S.J., R.M. Thorne, S. Bourdarie, I. de Pater, and B. Mauk. Jupiter's inner radiation belts. In: F. Bagenal, T. Dowling, and W. McKinnon, Editors. Jupiter: The Planet, Satellites and Magnetosphere, Cambridge Univ. Press, 671-688, 2004.

Bolton, S.J., F. Bagenal, M. Blanc, T. Cassidy, E. Chané, et al. Jupiter's magnetosphere: plasma sources and transport. Space Sci. Rev., 192 (1-4), 209-236, 2015, DOI: $10.1007 / \mathrm{s} 11214-015-0184-5$.

Bombardieri, D.J., M.L. Duldig, J.E. Humble, and K.J. Michael. An improved model for relativistic solar proton acceleration applied to the 2005 January 20 and earlier events. Astrophys. J., 682 , 1315-1327, 2008

Bonfond, B., D. Grodent, J.-C. Gérard, A. Radioti, J. Saur, and S. Jacobsen. UV Io footprint leading spot: a key feature for understanding the UV Io footprint multiplicity? Geophys. Res. Lett., 35, L05107, 2008, DOI: 10.1029/2007GL032418.

Bonfond, B., M.F. Vogt, J.-C. Gérard, D. Grodent, A. Radioti, and V. Coumans. Quasi-periodic polar flares at Jupiter: a signature of 
pulsed dayside reconnections? Geophys. Res. Lett., 38, L02104, 2011, DOI: $10.1029 / 2010$ GL045981.

Borucki, W., Z. Levin, R. Whitten, and R. Keesee. Predicted electrical conductivity between 0 and $80 \mathrm{~km}$ in the Venusian atmosphere. Icarus, 321, 302-321, 1982.

Borucki, W.J. Comparison of Venusian lightning observations. Icarus, 52, 354-364, 1982,

DOI: $10.1016 / 0019-1035(82) 90118-X$.

Boudjemai, A., R. Hocine, and S. Guerionne. Space Environment Effect on Earth Observation Satellite Instruments. In: Recent Advances in Space Technologies (RAST), 2015 7th International Conference on, IEEE, 627-634, ISBN: 978-1-4673-7760-7, 2015, DOI: 10.1109/RAST.2015.7208419.

Bougher, S.W., T.E. Cravens, J. Grebowsky, and J. Luhmann. The aeronomy of Mars: characterization by MAVEN of the upper atmosphere reservoir that regulates volatile escape. Space Sci. Rev., 195, 423-456, 2015a, DOI: 10.1007/s11214-014-0053-7.

Bougher, S.W., D.J. Pawlowski, J.M. Bell, S. Nelli, T. McDunn, J.R. Murphy, M. Chizek, and A.J. Ridley, Mars global ionospherethermosphere model (MGITM): solar cycle, seasonal, and diurnal variations of the Mars upper atmosphere. J. Geophys. Res. [Planets], 120, 311-342, 2015b.

Brace, L.H., H.A. Taylor, T.I. Gombosi, A.J. Kliore, W.C. Knudsen, and A.F. Nagy. The ionosphere of Venus - observations and their interpretation in Venus. University of Arizona Press, Tucson, USA, 779-840, 1983.

Brain, D.A., M. Bruce, and R. Jakosky. Atmospheric loss since the onset of the Martian geologic record: combined role of impact erosion and sputtering. J. Geophys. Res., 103, 22689-22694, 1998.

Brain, D.A., F. Bagenal, M.H. Acuña, and J.E.P. Connerney. Martian magnetic morphology: contributions from the solar wind and crust. J. Geophys. Res., 108 (A12), 1424, 2003,

DOI: 10.1029/2002JA009482.

Brain, D.A., S.W. Bougher, S.H. Brecht, G.M. Chanteur, S. Curry, et al. Comparison of global models for the escape of Martian atmospheric plasma. In: AGU Fall Meeting Abstracts, 1969, 2012.

Brandt, P.C., S. Barabash, E.C. Roelof, and C.J. Chase. Energetic neutral atom imaging at low altitudes from the Swedish microsatellite Astrid: observations at low $(\leq 10 \mathrm{keV})$ energies. J. Geophys. Res., 106, 24663-24674, 2001.

Brecht, A.S., and S.W. Bougher. Dayside thermal structure of Venus' upper atmosphere characterized by a global model. J. Geophys. Res., 117, E08002, 2012, DOI: $10.1029 / 2012$ JE004079.

Brice, N., and T.R. McDonough. Jupiter's radiation belts. Icarus, 18, 206-219, 1973, DOI: 10.1016/0019-1035(73)90204-2.

Broadfoot, A.L., S.K. Atreya, J.L. Bertaux, J.E. Blamont, A.J. Dessler, et al. Ultraviolet spectrometer observations of Neptune and Triton. Science, 246, 1459-1466, 1989 , DOI: $10.1126 /$ science.246.4936.1459.

Brown, R.H., R.N. Clark, B.J. Buratti, D.P. Cruikshank, J.W. Barnes, et al. Composition and physical properties of Enceladus' surface. Science, 311, 1425-1428, 2006, DOI: 10.1126/science.1121031.

Bunce, E.J., S.W.H. Cowley, and T.K. Yeoman. Jovian cusp processes: implications for the polar aurora. J. Geophys. Res. [Space Phys.], 109, A09S13, 2004,

DOI: 10.1029/2003JA010280.

Bunce, E.J., S.W.H. Cowley, and S.E. Milan. Interplanetary magnetic field control of Saturn's polar cusp aurora. Ann. Geophys., 23, 1405-1431, 2005,

DOI: 10.5194/angeo-23-1405-2005.

Bunce, E.J., C.S. Arridge, S.W.H. Cowley, and M.K. Dougherty. Magnetic field structure of Saturn's dayside magnetosphere and its mapping to the ionosphere: results from ring current modeling. J. Geophys. Res. [Space Phys.], 113, A02207, 2008, DOI: $10.1029 / 2007 J A 012538$.

Buratti, B.J., K. Soderlund, J. Bauer, J.A. Mosher, M.D. Hicks, et al. Infrared $(0.83-5.1 \mu \mathrm{m})$ photometry of Phoebe from the Cassini visual infrared mapping spectrometer. Icarus, 193, 309-322, 2008, DOI: 10.1016/j.icarus.2007.09.014.

Burger, M.H., R.M. Killen, W.E. McClintock, A.W. Merkel, R.J. Vervack, T.A. Cassidy, and M. Sarantos. Seasonal variations in Mercury's dayside calcium exosphere. Icarus, 238, 51-58, 2014.

Burke, B.F., and K.L. Franklin. Observations of a Variable radio source associated with the planet Jupiter. J. Geophys. Res. [Space Phys.], 60, 213-217, 1955, DOI: 10.1029/JZ060i002p00213.

Burlaga, L.F. Magnetic fields and plasmas in the inner heliosphere: helios results. Planet. Space Sci., 49 (14-15), 1619-1627, 2001,

Calvin, W.M., R.N. Clark, R.H. Brown, and J.R. Spencer. Spectra of the icy Galilean satellites from 0.2 to $5 \mu \mathrm{m}$ : a compilation, new observations, and a recent summary. J. Geophys. Res., 100, 19041-19048, 1995.

Carlson, R.W. A tenuous carbon dioxide atmosphere on Jupiter's moon Callisto. Science, 283, 820-821, 1999.

Carr, M.H., and J.W. Head. Oceans on Mars: an assessment of the observational evidence and possible fate. J. Geophys. Res. [Planets], 108, 5042, 2003.

Cassidy, T.A., and R.E. Johnson. Collisional spreading of Enceladus' neutral cloud. Icarus, 209, 696-703, 2010, DOI: 10.1016/j.icarus.2010.04.010.

Cassidy, T.A., R.E. Johnson, P.E. Geissler, and F. Leblanc. Simulation of $\mathrm{Na} \mathrm{D}$ emission near Europa during eclipse. J. Geophys. Res. [Space Phys.], 113, E02005, 2008, DOI: $10.1029 / 2007 J E 002955$.

Cassidy, T., P. Coll, F. Raulin, R.W. Carlson, R.E. Johnson, M.J. Loeffler, K.P. Hand, and R.A. Baragiola. Radiolysis and photolysis of icy satellite surfaces: experiments and theory. Space Sci. Rev., 153, 299-315, 2010, DOI: 10.1007/s11214-009-9625-3.

Cassidy, T.A., C.P. Paranicas, J.H. Shirley, J.B. Dalton III, B.D. Teolis, R.E. Johnson, L. Kamp, and A.R. Hendrix. Magnetospheric ion sputtering and water ice grain size at Europa. Planet. Space Sci., 77, 64-73, 2013, DOI: 10.1016/j.pss.2012.07.008.

Cassidy, T.A., A.W. Merkel, M.H. Burger, M. Sarantos, R.M. Killen, W.E. McClintock, and R.J. Vervack. Mercury's seasonal sodium exosphere: MESSENGER orbital observations. Icarus, 248 , 547-559, 2015, DOI: 10.1016/j.icarus.2014.10.037.

Chamberlain, J.W. Planetary coronae and atmospheric evaporation, Planet. Space Sci., 11, 901-960, 1963.

Chané, E., J. Saur, and S. Poedts. Modeling Jupiter's magnetosphere: influence of the internal sources. J. Geophys. Res. [Space Phys], 118, 2157-2172, 2013, DOI: 10.1002/jgra.50258.

Chappell, C.R. The role of the ionosphere in providing plasma to the terrestrial magnetosphere - an historical overview. Space Sci. Rev., 192 (1-4), 5-25, 2015.

Chassefiere, E., and F. Leblanc. Mars atmospheric escape and evolution; interaction with the solar wind. Planet. Space Sci., 52, 1039-1058, 2004.

Chaufray, J.Y., R. Modolo, F. Leblanc, G. Chanteur, R.E. Johnson, and J.G. Luhmann. Mars solar wind interaction: formation of the Martian corona and atmospheric loss to space. J. Geophys. Res. [Planets], 112, 9009, 2007.

Cheng, A.F., S.M. Krimigis, B.H. Mauk, E.P. Keath, C.G. Maclennan, L.J. Lanzerotti, M.T. Paonessa, and T.P. Armstrong. Energetic ion and electron phase space densities in the magnetosphere of Uranus. J. Geophys. Res. [Space Phys.], 92, $15315-15328,1987$.

Cheng, A.F., S.M. Krimigis, and L.J. Lanzerotti. Energetic particles at Uranus. In: J.T. Bergstralh, E.D. Miner, and M.S. Matthews, Editors. Uranus, Univ. of Arizona Press, USA, 831-893, 1991.

Clark, R.N., F.P. Fanale, and M.J. Gaffey. Surface composition of satellites. In: J. Burns, and M.S. Matthews, Editors. Satellites, University of Arizona Press, Tucson, 437-491, 1986.

Clark, R.N., R.H. Brown, R. Jaumann, D.P. Cruikshank, R.M. Nelson, et al. Compositional maps of Saturn's moon Phoebe from imaging spectroscopy. Nature, 435, 66-69, 2005, DOI: $10.1038 /$ nature 03558 . 
Clark, R.N., J.M. Curchin, R. Jaumann, D.P. Cruikshank, R.H. Brown, et al. Compositional mapping of Saturn's satellite Dione with Cassini VIMS and implications of dark material in the Saturn system. Icarus, 193, 372-386, 2008,

DOI: $10.1016 /$ j.icarus.2007.08.035.

Clark, R.N., D.P. Cruikshank, R. Jaumann, R.H. Brown, J.M. Curchin, et al. The composition of Iapetus: mapping results from Cassini VIMS. Icarus, 218, 831-860, 2012, DOI: 10.1016/j.icarus.2012.01.008.

Clarke, J.T., M.K. Hudson, and Y.L. Yung. The excitation of the far ultraviolet electroglow emissions on Uranus, Saturn, and Jupiter. J. Geophys. Res. [Space Phys.], 92 (A13), 15139-15147, 1987.

Clarke, J.T., J. Ajello, G. Ballester, L. Ben Jaffel, J. Connerney, et al. Ultraviolet emissions from the magnetic footprints of Io, Ganymede and Europa on Jupiter. Nature, 415, 997-1000, 2002.

Clarke, J.T., J. Nichols, J.C. Gérard, D. Grodent, K.C. Hansen, et al. Response of Jupiter's and Saturn's auroral activity to the solar wind. J. Geophys. Res. [Space Phys.], 114, A05210, 2009, DOI: 10.1029/2008JA013694.

Clifford, S.M., and T.J. Parker. The evolution of the Martian hydrosphere. AGU Fall Meeting Abstracts, 2001.

Cliver, E.W. The unusual relativistic solar proton events of 1979 August 21 and 1981 May 10. Astrophys. J., 639, 1206-1217, 2006, DOI: $10.1086 / 499765$.

Coates, A.J., H.J. McAndrews, A.M. Rymer, D.T. Young, F.J. Crary, et al. Plasma electrons above Saturn's main rings: CAPS observations. Geophys. Res. Lett., 32, L14S09, 2005 , DOI: $10.1029 / 2005$ GL022694.

Coates, A.J., F.J. Crary, G.R. Lewis, D.T. Young, J.H. Waite, and E.C. Sittler. Discovery of heavy negative ions in Titan's ionosphere. Geophys. Res. Lett., 34, L22103, 2007, DOI: $10.1029 / 2007$ GL030978.

Coates, A.J., A. Wellbrock, G.R. Lewis, G.H. Jones, D.T. Young, F.J. Crary, and J.H. Waite. Heavy negative ions in Titan's ionosphere: altitude and latitude dependence. Planet. Space Sci., 57 (14-15), 1866-1871, 2009.

Coates, A.J., S.M.E. Tsang, A. Wellbrock, R.A. Frahm, J.D. Winningham, S. Barabash, R. Lundin, D.T. Young, and F.J. Crary. Ionospheric photoelectrons: comparing Venus, Earth, Mars and Titan. Planet. Space Sci., 59 (10), 1019-1027, 2011.

Coates, A.J., A. Wellbrock, R.A. Frahm, J.D. Winningham, A. Fedorov, S. Barabash, and R. Lundin. Distant ionospheric photoelectron energy peak observations at Venus. Planet. Space Sci., 113, 378-384, 2015.

Connerney, J.E.P., M.H. Acuña, and N.F. Ness. Saturn's ring current and inner magnetosphere. Nature, 292, 724-726, 1981.

Connerney, J.E.P., M.H. Acuña, and N.F. Ness. Currents in Saturn's magnetosphere, J. Geophys. Res., 88, (A11), 8779-8789, 1983.

Connerney, J.E.P., M.H. Acuña, and N.F. Ness. The magnetic field of Neptune. J. Geophys. Res. [Space Phys.], 96, 19023-19042, 1991.

Cooper, J.F. Nuclear cascades in Saturn's rings - cosmic ray albedo neutron decay and origins of trapped protons in the inner magnetosphere. J. Geophys. Res. [Space Phys.], 88, 3945-3954, 1983, DOI: 10.1029/JA088iA05p03945.

Coradini, A., F. Tosi, A.I. Gavrishin, F. Capaccioni, P. Cerroni, et al Identification of spectral units on Phoebe. Icarus, 193, 233-251, 2008, DOI: 10.1016/j.icarus.2007.07.023.

Coradini, A., D. Turrini, C. Federico, and G. Magni. Vesta and Ceres: crossing the history of the Solar System. Space Sci. Rev., 163, 25-40, 2011.

Coustenis, A., and F.W. Taylor. Titan: exploring an earthlike world, 2nd edn., World Scientific Publishing Co, USA, 2008.

Coustenis, A., A. Salama, E. Lellouch, T. Encrenaz, G.L. Bjoraker, R.E. Samuelson, T. de Graauw, H. Feuchtgruber, and M.F. Kessler. Evidence for water vapor in Titan's atmosphere from ISO/SWS data. $A \& A$, 336, L85-L89, 1998.

Coustenis, A., T. Tokano, M.H. Burger, T.A. Cassidy, R.M. Lopes, R.D. Lorenz, K.D. Retherford, and G. Schubert. Atmospheres/ exospheres characteristics of icy satellites. Space Sci. Rev., 153, 155-184, 2010.

Coustenis, A., D.E. Jennings, R.K. Achterbergh, G. Bampasidis, P. Lavvas, C.A. Nixon, N.A. Teanby, C.M. Anderson, and F.M. Flasar. Titan's temporal evolution in stratospheric trace gases near the poles. Icarus, 270, 409-420, 2016,

DOI: 10.1016/j.icarus.2015.08.027.

Cowley, S., E. Bunce, and R. Prangé. Saturn's polar ionospheric flows and their relation to the main auroral oval. Ann. Geophys., 22, 1379-1394, 2004, DOI: 10.5194/angeo-22-1379-2004.

Cowley, S.W.H. Response of Uranus' auroras to solar wind compressions at equinox. J. Geophys. Res. [Space Phys.], 118 2897-2902, 2013, DOI: 10.1002/jgra.50323.

Cowley, S.W.H., and E.J. Bunce. Origin of the main auroral oval in Jupiter's coupled magnetosphere-ionosphere system. Planet. Space Sci., 49, 1067-1088, 2001, DOI: $10.1016 / \mathrm{S} 0032-0633(00) 00167-7$

Cowley, S.W.H., and E.J. Bunce. Corotation-driven magnetosphereionosphere coupling currents in Saturn's magnetosphere and their relation to the auroras. Ann. Geophys., 21, 1691-1707, 2003, DOI: 10.5194/angeo-21-1691-2003.

Cowley, S.W.H., S.V. Badman, E.J. Bunce, J.T. Clarke, J.-C. GéRard, D. Grodent, C.M. Jackman, S.E. Milan, and T.K. Yeoman. Reconnection in a rotation-dominated magnetosphere and its relation to Saturn's auroral dynamics. J. Geophys. Res. [Space Phys.], 110, A02201, 2005, DOI: 10.1029/2004JA010796.

Cravens, T.E., I.P. Robertson, S.A. Ledvina, D. Mitchell, S.M Krimigis, and J.H. Waite. Energetic ion precipitation at Titan. Geophys. Res. Lett., 35, L03103, 2008, DOI: $10.1029 / 2007$ GL032451.

Cravens, T.E., R.L. McNutt, J.H. Waite, I.P. Robertson, J.G. Luhmann, W. Kasprzak, and W.-H. Ip. Plume ionosphere of Enceladus as seen by the Cassini ion and neutral mass spectrometer. Geophys. Res. Lett., 36, L08106, 2009, DOI: 10.1029/2009GL037811.

Cruikshank, D.P., E. Wegryn, C.M. Dalle Ore, R.H. Brown, J.-P. Bibring, et al. Hydrocarbons on Saturn's satellites Iapetus and Phoebe. Icarus, 193, 334-343, 2008, DOI: $10.1016 /$ j.icarus.2007.04.036.

Cruikshank, D.P., A.W. Meyer, R.H. Brown, R.N. Clark, R. Jaumann, et al. Carbon dioxide on the satellites of Saturn: results from the Cassini VIMS investigation and revisions to the VIMS wavelength scale. Icarus, 206, 561-572, 2010, DOI: 10.1016/j.icarus.2009.07.012.

Cui, J., R.V. Yelle, V. Vuitton, J.H. Waite Jr., W.T. Kasprzak, et al. Analysis of Titan's neutral upper atmosphere from Cassini Ion Neutral Mass Spectrometer measurements. Icarus, 200, 581-615, 2009a, DOI: 10.1016/j.icarus.2008.12.005.

Cui, J., M. Galand, R.V. Yelle, V. Vuitton, J.-E. Wahlund, et al. Diurnal variations of Titan's ionosphere. J. Geophys. Res., 114, A06310, 2009b, DOI: 10.1029/2009JA014228.

Cunningham, N.J., J.R. Spencer, P.D. Feldman, D.F. Strobel, K. France, and S.N. Osterman. Detection of Callisto's oxygen atmosphere with the Hubble Space Telescope. Icarus, 254, 178-189, 2015, DOI: 10.1016/j.icarus.2015.03.021.

Dalton, B., D. Cruikshank, K. Stephan, T. McCord, A. Coustenis, R. Carlson, and A. Coradini. Chemical composition of icy satellite surfaces. Space Sci. Rev., 153, 113-154, 2010.

Dandouras, I., P. Garnier, D.G. Mitchell, E.C. Roelof, P.C. Brandt, N. Krupp, and S.M. Krimigis. Titan's exosphere and its interaction with Saturn's magnetosphere. Philos. Trans. R. Soc. London: Ser. A, 367, 743-752, 2009,

DOI: $10.1098 /$ rsta.2008.0249.

Dartnell, L.R. Ionizing radiation and life. Astrobiology, 11, 551-582, 2011, DOI: 10.1089/ast.2010.0528.

Dartnell, L.R., L. Desorgher, J.M. Ward, and A.J. Coates. Martian sub-surface ionising radiation: biosignatures and geology. Biogeosciences, 4, 545-558, 2007a,

DOI: $10.5194 / \mathrm{bg}-4-545-2007$. 
Dartnell, L.R., L. Desorgher, J.M. Ward, and A.J. Coates. Modelling the surface and subsurface Martian radiation environment: implications for astrobiology. Geophys. Res. Lett., 34, L02207, 2007b, DOI: 10.1029/2006GL027494.

Dartnell, L.R., S.J. Hunter, K.V. Lovell, A.J. Coates, and J.M. Ward. Low-temperature ionizing radiation resistance of Deinococcus radiodurans and Antarctic Dry Valley bacteria. Astrobiology, 10, 717-732, 2010, DOI: 10.1089/ast.2009.0439.

Dartnell, L.R., T.A. Nordheim, M.R. Patel, J.P. Mason, A.J. Coates, and G.H. Jones. Constraints on a potential aerial biosphere on Venus: I. Cosmic rays. Icarus, 257, 396-405, 2015.

Decker, R.B., and A.F. Cheng. A model of Triton's role in Neptune's magnetosphere. J. Geophys. Res. [Space Phys.], 99, 19027, 1994, DOI: 10.1029/94JE01867.

Delamere, P.A., and F. Bagenal. Magnetotail structure of the giant magnetospheres: implications of the viscous interaction with the solar wind. J. Geophys. Res. [Space Phys.], 118 (11), 7045-7053, 2013.

Delamere, P.A., F. Bagenal, and A. Steffl. Radial variations in the Io plasma torus during the Cassini era. J. Geophys. Res. [Space Phys.], 110, A12223, 2005, DOI: 10.1029/2005JA011251.

Delcourt, D.C., S. Grimald, F. Leblanc, J.-J. Berthelier, A. Millilo, A. Mura, S. Orsini, and T.E. Moore. A quantitative model of the planetary $\mathrm{Na}^{+}$contribution to Mercury's magnetosphere. Ann. Geophys., 21 (8), 1723-1736, 2003.

DiBraccio, G.A., J.A. Slavin, S.A. Boardsen, B.J. Anderson, H. Korth, et al. MESSENGER observations of magnetopause structure and dynamics at Mercury. J. Geophys. Res. [Space Phys.], 118, 997-1008, 2013, DOI: 10.1002/jgra.50123.

Dols, V.J., F. Bagenal, T.A. Cassidy, F.J. Crary, and P.A. Delamere. Europa's atmospheric neutral escape: Importance of symmetrical $\mathrm{O}_{2}$ charge exchange. Icarus, 264, 387-397, 2016.

Donahue, T.M., D.H. Grinspoon, R.E. Hartle, and R.R. Hodgee. Ion/neutral escape of hydrogen and deuterium: evolution of water. In: S.W. Bougher, D.M. Hunten, and R.J. Philips, Editors. Venus II - Geology, Geophysics, Atmosphere, and Solar Wind Environment, University of Arizona Press, Tuscon, 385-414, 1997.

Dorman, L. Cosmic Rays in Magnetospheres of the Earth and other Planets, 358, Springer Science \& Business Media, Germany, 2009.

Dougherty, M.K., L.W. Esposito, and S.M. Krimigis. Saturn from Cassini-Huygens, Springer, Germany, 2009, DOI: 10.1007/978-1-4020-9217-6.

Drossart, P., B. Bézard, S.K. Atreya, J. Bishop, J.H. Waite, and D. Boice. Thermal profiles in the auroral regions of Jupiter. J. Geophys. Res. [Planets], 98 (E10), 18803-18811, 1993.

Drossart, P., G. Piccioni, J.-C. Gérard, M.A. Lopez-Valverde, A. Sanchez-Lavega, et al. A dynamic upper atmosphere of Venus as revealed by VIRTIS on Venus Express. Nature, 450, 641-645, 2007.

Dubach, J., R. Whitten, and J. Sims. The lower ionosphere of Venus. Planet. Space Sci., 22, 525-536, 1974, DOI: $10.1016 / 0032-0633(74) 90087-7$.

Dubinin, E., R. Modolo, M. Fraenz, J. Woch, G. Chanteur, et al. Plasma environment of Mars as observed by simultaneous MEX-ASPERA-3 and MEX-MARSIS observations. J. Geophys. Res. [Space Phys], 113, 10217, 2008.

Dudok de Wit, T., M. Kretzschmar, J. Lilensten, and T. Woods. Finding the best proxies for the solar UV irradiance. Geophys. Res. Lett., 36, 10107, 2009, DOI: 10.1029/2009GL037825.

Dungey, J.W. Interplanetary magnetic field and the auroral zones. Phys. Rev. Lett., 6, 47-48, 1961 DOI: 10.1103/PhysRevLett.6.47.

Dušík, Š., G. Granko, J. Šafránková, Z. Němeček, and K. Jelínek. IMF cone angle control of the magnetopause location: statistical study. Geophys. Res. Lett., 37, L19103, 2010,

DOI: $10.1029 / 2010$ GL044965.
Duvall, A.L., C.G. Justus, and V.W. Keller. Global Reference Atmospheric Models for Aeroassist Applications. In: $3 \mathrm{rd}$ international planetary probe workshop (Anavyssos, Attiki, Greece), 27, 20060004756 (NASA Marshall Space Flight Center, Huntsville, USA), 2005.

Edberg, N.J.T., J.-E. Wahlund, K. Ågren, M.W. Morooka, R. Modolo, C. Bertucci, and M.K. Dougherty. Electron density and temperature measurements in the cold plasma environment of Titan: implications for atmospheric escape. Geophys. Res. Lett., 37, L20105, 2010, DOI: 10.1029/2010GL044544.

Elsner, R.F., N. Lugaz, J.H. Waite, T.E. Cravens, G.R. Gladstone, et al. Simultaneous Chandra X ray, Hubble Space Telescope ultraviolet, and Ulysses radio observations of Jupiter's aurora. J. Geophys. Res. [Space Phys.], 110, A01207, 2005, DOI: 10.1029/2004JA010717.

Eviatar, A., V.M. Vasyliunas, and D.A. Gurnett. The ionosphere of Ganymede. Planet. Space Sci., 49, 327-336, 2001, DOI: $10.1016 / \mathrm{S} 00320633(00) 00154-9$.

Fairfield, D.H. Average and unusual locations of the Earth's magnetopause and bow shock. J. Geophys. Res., 76 (28), 6700, 1971.

Famà, M., J. Shi, and R.A. Baragiola. Sputtering of ice by low-energy ions. Surf. Sci., 602, 156-161, 2008.

Fasel, G.J., L.C. Lee, and R.W. Smith. A mechanism for the multiple brightenings of dayside poleward-moving auroral forms. Geophys. Res. Lett., 20, 2247-2250, 1993, DOI: 10.1029/93GL02487.

Fedorova, A., O. Korablev, A.-C. Vandaele, J.-L. Bertaux, D. Belyaev, et al. HDO and $\mathrm{H} 2 \mathrm{O}$ vertical distributions and isotopic ratio in the Venus mesosphere by solar occultation at infrared spectrometer on board Venus Express. J. Geophys. Res., 113, E00B22, 2008, DOI: 10.1029/2008JE003146.

Feuchtgruber, H., E. Lellouch, T. de Graauw, B. Bézard, T. Encrenaz, and M. Griffin. External supply of oxygen to the atmospheres of the giant planets. Nature, 389 (6647), 159-162, 1997.

Fichtner, H., B. Heber, and M. Leipold. The science with the interstellar heliopause probe. Astrophys. Space Sci. Trans., 2, 33-43, 2006.

Fleshman, B.L., P.A. Delamere, and F. Bagenal. Modeling the Enceladus plume-plasma interaction. Geophys. Res. Lett., 37, L03202, 2010, DOI: 10.1029/2009GL041613.

Forbes, J.M., S. Bruinsma, and F.G. Lemoine. Solar rotation effects on the thermospheres of Mars and Earth. Science, 312, 1366-1368, 2006, DOI: 10.1126/science.1126389.

Forget, F., F. Montmessin, J.-L. Bertaux, F. Gonzalez-Galindo, S. Lebonnois, E. Quemerais, A. Reberac, E. Dimarellis, and M.A. Lopez-Valverde. Density and temperatures of the upper Mar atmosphere measured by stellar occultations with Mars Express SPICAM. J. Geophys. Res. [Planets], 114, 1004, 2009.

Fox, J.L. Near-terminator Venus ionosphere: how Chapman-esque? J. Geophys. Res., 112, E04S02, 2007, DOI: $10.1029 / 2006 J E 002736$.

Fox, J.L. The chemistry of protonated species in the martian ionosphere. Icarus, 252, 366-392, 2015, DOI: $10.1016 /$ j.icarus.2015.01.010.

Fox, J.L., and S.W. Bougher. Structure, luminosity, and dynamics of the Venus thermosphere. In: Venus Aeronomy, Springer, Netherlands, 357-489, 1991.

Fox, J.L., P. Zhou, and S.W. Bougher. The Martian thermosphere/ ionosphere at high and low solar activities. Adv. Space Res., 17 (11), 203-218, 1996, DOI: 10.1016/0273-1177(95)00751-Y.

Frank, L.A., J.D. Craven, J.L. Burch, and J.D. Winningham. Polar views of the earth's aurora with Dynamics Explorer. Geophys. Res. Lett., 9, 1001-1004, 1982, DOI: $10.1029 /$ GL009i009p01001.

Frank, L.A., W.R. Paterson, K.L. Ackerson, and S.J. Bolton. Outflow of hydrogen ions from Ganymede. Geophys. Res. Lett., 24 (17), 2151-2154, 1997. 
Fulchignoni, M., F. Ferri, F. Angrilli, A.J. Ball, A. Bar-Nun, et al. In situ measurements of the physical characteristics of Titan's environment. Nature, 438, 785-791, 2005, DOI: 10.1038 /nature04314.

Funsten, H.O., F. Allegrini, G.B. Crew, R. DeMajistre, P.C. Frisch, et al. Structures and spectral variations of the outer heliosphere in IBEX energetic neutral atom maps. Science, 326, 5955-5964, 2009.

Fuselier, S.A., H.L. Collin, A.G. Ghielmetti, E.S. Claflin, T.E. Moore, et al. Localized ion outflow in response to a solar wind pressure pulse. J. Geophys. Res. [Space Phys.], 107, 1203, 2002, DOI: 10.1029/2001JA000297.

Futaana, Y., S. Barabash, M. Yamauchi, S. McKenna-Lawlor, R. Lundin, et al. Mars Express and Venus Express multi-point observations of geoeffective solar flare events in December 2006. Planet. Space Sci., 6, 873-880, 2008.

Futaana, Y., S. Barabash, X.-D. Wang, M. Wieser, G.S. Wieser, P. Wurz, N. Krupp, and P.C. Brandt. Low-energy energetic neutral atom imaging of Io plasma and neutral tori. Planet. Space Sci., 108, 41-53, 2015.

Gagné, M.-Ė., J.-L. Bertaux, F. González-Galindo, S.M.L. Melo, F. Montmessin, and K. Strong. New nitric oxide (NO) nightglow measurements with SPICAM/MEx as a tracer of Mars upper atmosphere circulation and comparison with LMD-MGCM model prediction: evidence for asymmetric hemispheres. J. Geophys. Res. [Planets], 118, 2172-2179, 2013, DOI: $10.1002 /$ jgre. 20165 .

Galand, M., J. Lilensten, D. Toublanc, and S. Maurice. The ionosphere of Titan: ideal diurnal and nocturnal cases. Icarus, 140, 92-105, 1999, DOI: 10.1006/icar.1999.6113.

Galand, M., L. Moore, B. Charnay, I. Mueller-Wodarg, and M. Mendillo. Solar primary and secondary ionization at Saturn. J. Geophys. Res., 114 (A6), A06313, 2009.

Gannon, J.L., X. Li, and M. Temerin. Parametric study of shockinduced transport and energization of relativistic electrons in the magnetosphere. J. Geophys. Res., 110, A12206, 2005, DOI: 10.1029/2004JA010679.

Garnier, P., J.-E. Wahlund, L. Rosenqvist, R. Modolo, K. Agren, et al. Titan's ionosphere in the magnetosheath: Cassini RPWS results during the T32 flyby. Ann. Geophys., 27, 4257-4272, 2009, DOI: 10.5194/angeo-27-4257-2009.

Garnier, P., I. Dandouras, D. Toublanc, E.C. Roelof, P.C. Brandt, et al. Statistical analysis of the energetic ion and ENA data for the Titan environment. Planet. Space Sci., 58, 1811-1822, 2010, DOI: $10.1016 /$ j.pss.2010.08.009.

Gérard, J.-C., D. Grodent, J. Gustin, A. Saglam, J.T. Clarke, and J.T. Trauger. Characteristics of Saturn's FUV aurora observed with the Space Telescope Imaging Spectrograph. J. Geophys. Res. [Space Phys.], 109, A09207, 2004, DOI: $10.1029 / 2004 J A 010513$.

Gérard, J.-C., E.J. Bunce, D. Grodent, S.W.H. Cowley, J.T. Clarke, and S.V. Badman. Signature of Saturn's auroral cusp: simultaneous Hubble Space Telescope FUV observations and upstream solar wind monitoring. J. Geophys. Res. [Space Phys.], 110, A11201, 2005, DOI: 10.1029/2005JA011094.

Gérard, J.C., L. Soret, L. Libert, R. Lundin, A. Stiepen, A. Radioti, and J.L. Bertaux. Concurrent observations of ultraviolet aurora and energetic electron precipitation with Mars Express. J. Geophys. Res. [Space Phys.], 120 (8), 6749-6765, 2015.

Gershman, D.J., J.A. Slavin, J.M. Raines, T. Zurbuchen, B. Anderson, H. Korth, D. Baker, and S. Solomon. Magnetic flux pileup and plasma depletion in Mercury's subsolar magnetosheath. J. Geophys. Res., 118, 7181, 2013.

Gillmann, C., P. Lognonné, E. Chassefiere, and M. Moreira. The present-day atmosphere of Mars: where does it come from? Earth Planet. Sci. Lett., 277, 384-393, 2009.

Gladstone, G.R., J.H. Waite, D. Grodent, W.S. Lewis, F.J. Crary, et al. A pulsating auroral X-ray hot spot on Jupiter. Nature, 415, 1000-1003, 2002.
Glassmeier, K.-H., J. Grosser, U. Auster, D. Constantinescu, Y. Narita, and S. Stellmach. Electromagnetic induction effects and dynamo action in the Hermean system. Space Sci. Rev., 132, 511-527, 2007, DOI: 10. 1007/s11214-007-9244-9.

Gombosi, T.I., T.P. Armstrong, C.S. Arridge, K.K. Khurana, S.M. Krimigis, N. Krupp, A.M. Persoon, and M.F. Thomsen. Saturn's Magnetospheric Configuration. In: M.K. Dougherty, L.W. Esposito, and S.M. Krimigis, Editors. Saturn from Cassini-Huygens, Springer Science+Business Media B.V, Heidelberg, 203, 2009.

Gopalswamy, N., H. Xie, S. Yashiro, S. Akiyama, P. Mäkelä, and I.G. Usoskin. Properties of ground level enhancement events and the associated solar eruptions during solar cycle 23. Space Sci. Rev., 171, 23-60, 2012, DOI: 10.1007/s11214-012-9890-4.

Gray, C.L., N.J. Chanover, T.G. Slanger, and K. Molaverdikhani. The effect of solar flares, coronal mass ejections, and solar wind streams on Venus' $5577 \AA$ oxygen green line. Icarus, 233, 342-347, 2014.

Grasset, O., M.K. Dougherty, A. Coustenis, E.J. Bunce, C. Erd, et al. JUpiter ICy moons Explorer (JUICE): an ESA mission to orbit Ganymede and to characterise the Jupiter system. Planet. Space Sci., 78, 1-21, 2013, DOI: 10.1016/j.pss.2012.12.002

Grodent, D., J.H. Waite, and J.C. Gérard. A self-consistent model of the Jovian auroral thermal structure. J. Geophys. Res. [Space Phys.], 106 (A7), 12933-12952, 2001.

Grodent, D., J.T. Clarke, J. Kim, J.H. Waite, and S.W.H. Cowley. Jupiter's main auroral oval observed with HST-STIS. J. Geophys. Res. [Space Phys.], 108, 1389, 2003a,

DOI: $10.1029 / 2003$ JA009921.

Grodent, D., J.T. Clarke, J.H. Waite, S.W.H. Cowley, J.-C. Gérard, and J. Kim. Jupiter's polar auroral emissions. J. Geophys. Res. [Space Phys.], 108, 1366, 2003b, DOI: 10.1029/2003JA010017.

Grodent, D., J.-C. Gérard, J.T. Clarke, G.R. Gladstone, and J.H. Waite. A possible auroral signature of a magnetotail reconnection process on Jupiter. J. Geophys. Res. [Space Phys.], 109, A05201, 2004, DOI: 10.1029/2003JA010341.

Grodent, D., J.-C. Gérard, S.W.H. Cowley, E.J. Bunce, and J.T. Clarke. Variable morphology of Saturn's southern ultraviolet aurora. J. Geophys. Res. [Space Phys.], 110, A07215, 2005, DOI: $10.1029 / 2004 J A 010983$.

Grodent, D., B. Bonfond, A. Radioti, J.-C. Gerard, X. Jia, J.D. Nichols, and J.T. Clarke. Auroral footprint of Ganymede. J. Geophys. Res. [Space Phys.], 114, A07212, 2009, DOI: 10.1029/2009JA014289.

Grodent, D., J. Gustin, J.-C. Gerard, A. Radioti, B. Bonfond, and W.R. Pryor. Small-scale structures in Saturn's ultraviolet aurora. J. Geophys. Res. [Space Phys.], 116, A09225, 2011,

DOI: $10.1029 / 2011$ JA016818.

Grodent, D.A. Brief review of ultraviolet auroral emissions on giant planets. Space Sci. Rev., 187, 23-50, 2015, DOI: $10.1007 / \mathrm{s} 11214-014-0052-8$.

Gronoff, G., J. Lilensten, C. Simon, M. Barthélemy, and F. Leblanc. Modelling the Venusian airglow. $A \& A, 482,1015-1029,2008$, DOI: 10.1051/0004-6361:20077503.

Gronoff, G., J. Lilensten, L. Desorgher, and E. Flückiger. Ionization processes in the atmosphere of Titan. I. Ionization in the whole atmosphere. $A \& A, \mathbf{5 0 6}, 955-964,2009$ a,

DOI: $10.1051 / 0004-6361 / 200912371$.

Gronoff, G., J. Lilensten, and R. Modolo. Ionization processes in the atmosphere of Titan. II. Electron precipitation along magnetic field lines. $A \& A$, 506, 965-970, 2009b,

DOI: $10.1051 / 0004-6361 / 200912125$.

Gronoff, G., C. Mertens, J. Lilensten, L. Desorgher, E. Flückiger, and P. Velinov. Ionization processes in the atmosphere of Titan. III. Ionization by high-Z nuclei cosmic rays. $A \& A, \mathbf{5 2 9}, \mathrm{A} 143$, 2011, DOI: $10.1051 / 0004-6361 / 201015675$.

Gronoff, G., R.B. Norman, and C.J. Mertens. Computation of cosmic ray ionization and dose at Mars. I: A comparison of HZETRN and Planetocosmics for proton and alpha particles. 
Adv. Space Res., 55, 1799-1805, 2015, DOI: 10.1016/j.asr.2015.01.028.

Grott, M., A. Morschhauser, D. Breuer, and E. Hauber. Volcanic outgassing of $\mathrm{CO}_{2}$ and $\mathrm{H}_{2} \mathrm{O}$ on Mars. Earth Planet. Sci. Lett., 308, 391-400, 2011

Guervilly, C., P. Cardin, and N. Schaeffer. A dynamo driven by zonal jets at the upper surface: applications to giant planets. Icarus, 218, 100-114, 2012, DOI: 10.1016/j.icarus.2011.11.014.

Guinan, E.F., and I. Ribas. Our changing Sun: the role of solar nuclear evolution and magnetic activity on Easrth's atmosphere and climate. ASP Conference Series, 269, 86-106, 2002.

Gurnett, D.A., W.S. Kurth, A. Roux, S.J. Bolton, and C.F. Kennel. Evidence for a magnetosphere at Ganymede from plasma-wave observations by the Galileo spacecraft. Nature, 384, 535-537, 1996, DOI: $10.1038 / 384535 \mathrm{a} 0$.

Gurnett, D.A., P. Zarka, R. Manning, W.S. Kurth, G.B. Hospodarsky, T.F. Averkamp, M.L. Kaiser, and W.M. Farrell. Non-detection at Venus of high-frequency radio signals characteristic of terrestrial lightning. Nature, 409, 313-315, 2001, DOI: 10.1038/35053009.

Gurnett, D.A., W.S. Kurth, G.B. Hospodarsky, A.M. Persoon, P. Zarka, et al. Control of Jupiter's radio emission and aurorae by the solar wind. Nature, 415 (6875), 985-987, 2002.

Gurtner, M., L. Desorgher, E.O. Flückiger, and M.R. Moser. A Geant4 application to simulate the interaction of space radiation with the Mercurian environment. Adv. Space Res., 37, 1759-1763, 2006, DOI: 10.1016/j.asr.2004.12.015.

Hall, D.T., D.F. Strobel, P.D. Feldman, M.A. McGrath, and H.A. Weaver. Detection of an oxygen atmosphere on Jupiter's moon Europa. Nature, 373 (6516), 677-681, 1995.

Hall, D.T., P.D. Feldman, M.A. McGrath, and D.F. Strobel. The far ultraviolet oxygen airglow of Europa and Ganymede. Astrophys. J., 499, 475-481, 1998 .

Hallinan, G., S.P. Littlefair, G. Cotter, S. Bourke, L.K. Harding, et al. Magnetospherically driven optical and radio aurorae at the end of the stellar main sequence. Nature, 523 (7562), 568-571, 2015.

Hamelin, M., C. Béghin, R. Grard, J.J. López-Moreno, K. Schwingenschuh, et al. Electron conductivity and density profiles derived from the mutual impedance probe measurements performed during the descent of Huygens through the atmosphere of Titan. Planet. Space Sci., 55, 1964-1977, 2007, DOI: 10.1016/j.pss.2007.04.008.

Hanel, R., B. Conrath, F.M. Flasar, V. Kunde, W. Maguire, et al. Infrared observations of the Uranian system. Science, 233 (4759), 70-74, 1986.

Hanlon, P.G., M.K. Dougherty, R.J. Forsyth, M.J. Owens, K.C. Hansen, G. Tóth, F.J. Crary, and D.T. Young. On the evolution of the solar wind between 1 and $5 \mathrm{AU}$ at the time of the Cassini Jupiter flyby: multispacecraft observations of interplanetary coronal mass ejections including the formation of a merged interaction region. J. Geophys. Res. [Space Phys.], 109 (A9), A09S03, 2004

Hansen, C.J., D.E. Shemansky, and A.R. Hendrix. Cassini UVIS observations of Europa's oxygen atmosphere and torus. Icarus, 176, 305-315, 2005 .

Hartogh, P., E. Lellouch, J. Crovisier, M. Banaszkiewicz, F. Bensch, et al. Water and related chemistry in the Solar System. A guaranteed time key programme for Herschel. Planet. Space Sci., 57 (13), 1596-1606, 2009.

Hartogh, P., D.C. Lis, D. Bockelée-Morvan, M. de Val-Borro, and N. Biver. Ocean-like water in the Jupiter-family comet $103 \mathrm{P} /$ Hartley 2. Nature, 478 (7368), 218-220, 2011.

Hasegawa, H., M. Fujimoto, Y. Saito, and T. Mukai. Dense and stagnant ions in the low-latitude boundary region under northward interplanetary magnetic field. Geophys. Res. Lett., 31, L06802, 2004a, DOI: 10.1029/2003GL019120.

Hasegawa, H., M. Fujimoto, T.-D. Phan, H. Reme, A. Balogh, M.W. Dunlop, C. Hashimoto, and R. TanDokoro. Transport of solar wind into Earth's magnetosphere through rolled-up KelvinHelmholtz vortices. Nature, 430, 755-758, 2004b.
Hassler, D.M., C. Zeitlin, R.F. Wimmer-Schweingruber, S. Böttcher, C. Martin, et al. The Radiation Assessment Detector (RAD) investigation. Space Sci. Rev., 170, 503-558, 2012, DOI: $10.1007 / \mathrm{s} 11214-012-9913-1$

Hassler, D.M., C. Zeitlin, R.F. Wimmer-Schweingruber, B. Ehresmann, S. Rafkin, et al. Mars' surface radiation environment measured with the Mars Science Laboratory's Curiosity rover. Science, 343, 1244797, 2014,

DOI: $10.1126 /$ science. 1244797.

Herbert, F. Aurora and magnetic field of Uranus. J. Geophys. Res. [Space Phys] , 114, A11, 2009, DOI: 10.1029/2009JA014394.

Herbert, F., B.R. Sandel, R.V. Yelle, J.B. Holberg, A.L. Broadfoot, D.E. Shemansky, S.K. Atreya, and P.N. Romani. The upper atmosphere of Uranus - EUV occultations observed by Voyager 2. J. Geophys. Res., 92, 15093-15109, 1987.

Hill, T.W. The Jovian auroral oval. J. Geophys. Res. [Space Phys.], 106, 8101-8108, 2001, DOI: 10.1029/2000JA000302.

Ho, G.C., S.M. Krimigis, R.E. Gold, D.N. Baker, B.J. Anderson, et al. Spatial distribution and spectral characteristics of energetic electrons in Mercury's magnetosphere. J. Geophys. Res., 117, A00M04, 2012.

Hood, L.L. Radial diffusion in Saturn's radiation belts - a modeling analysis assuming satellite and ring E absorption. J. Geophys. Res. [Space Phys.], 88, 808-818, 1983, DOI: $10.1029 / \mathrm{JA} 088 \mathrm{iA02p00808.}$

Hood, L.L., and G. Schubert. Inhibition of solar wind impingement on Mercury by planetary induction currents. J. Geophys. Res., 84, 2641-2647, 1979.

Huddleston, D.E., C.T. Russell, G. Le, and A. Szabo. Magnetopause structure and the role of reconnection at the outer planets. J. Geophys. Res. [Space Phys.], 102, 24289-24302, 1997, DOI: 10.1029/97JA02416.

Hunt, G.J., S.W.H. Cowley, G. Provan, E.J. Bunce, I.I. Alexeev, E.S. Belenkaya, V.V. Kalegaev, M.K. Dougherty, and A.J. Coates. Field-aligned currents in Saturn's southern nightside magnetosphere: subcorotation and planetary period oscillation components. J. Geophys. Res. [Space Phys.], 119, 9847-9899, 2014, DOI: 10.1002/2014JA020506.

Ip, W.-H. Europa's oxygen exosphere and its magnetospheric interaction. Icarus, 120, 317-325, 1996, DOI: 10.1006/icar.1996.0052.

Jackman, C.M., N. Achilleos, E.J. Bunce, S.W.H. Cowley, M.K. Dougherty, G.H. Jones, S.E. Milan, and E.J. Smith. Interplanetary magnetic field at $\sim 9$ AU during the declining phase of the solar cycle and its implications for Saturn's magnetospheric dynamics. J. Geophys. Res., 109, A11203, 2004 DOI: $10.1029 / 2004 J A 010614$

Jackman, C.M., R.J. Forsyth, and M.K. Dougherty. The overall configuration of the interplanetary magnetic field upstream of Saturn as revealed by Cassini observations. J. Geophys. Res., 113, A08114, 2008, DOI: 10.1029/2008JA013083.

Jackman, C.M., L. Lamy, M.P. Freeman, M.P. Freeman, P. Zarka, B. Cecconi, W.S. Kurth, S.W.H. Cowley, and M.K. Dougherty. On the character and distribution of lower-frequency radio emissions at Saturn and their relationship to substorm-like events. J. Geophys. Res., 114, A08211, 2009, DOI: $10.1029 / 2008 J A 013997$

Jackman, C.M., N. Achilleos, S.W.H. Cowley, E.J. Bunce, A. Radioti, D. Grodent, S.V. Badman, M.K. Dougherty, and W. Pryor. Auroral counterpart of magnetic field dipolarizations in Saturn's tail. Planet. Space Sci., 82, 34-42, 2013, DOI: $10.1016 /$ j.pss.2013.03.010.

Jakosky, B.M., J.M. Grebowsky, J.G. Luhmann, J. Connerney, F. Eparvier, et al. MAVEN observations of the response of Mars to an interplanetary coronal mass ejection. Science, 350 (6261), $1-7,2015$.

Jasinski, J.M., C.S. Arridge, L. Lamy, J.S. Leisner, M.F. Thomsen, et al. Cusp observation at Saturn's high-latitude magnetosphere by the Cassini spacecraft. Geophys. Res. Lett., 41, 1382-1388, 2014, DOI: 10.1002/2014GL059319. 
Jaumann, R., R.H. Brown, K. Stephan, J.W. Barnes, and L.A. Soderblom. Fluvial erosion and post-erosional processes on Titan. Icarus, 197, 526-538, 2008, DOI: $10.1016 /$ j.icarus.2008.06.002.

Jia, X., R.J. Walker, M.G. Kivelson, K.K. Khurana, and J.A. Linker. Properties of Ganymede's magnetosphere inferred from improved three-dimensional MHD simulations. J. Geophys. Res. [Space Phys.], 114, A09209, 2009, DOI: 10.1029/2009JA014375.

Jia, X., R.J. Walker, M.G. Kivelson, K.K. Khurana, and J.A. Linker. Dynamics of Ganymede's magnetopause: intermittent reconnection under steady external conditions. J. Geophys. Res., 115, A12202, 2010, DOI: 10.1029/ 2010JA015771.

Jia, X., K.C. Hansen, T.I. Gombosi, M.G. Kivelson, G. Tóth, D.L. DeZeeuw, and A.J. Ridley. Magnetospheric configuration and dynamics of Saturn's magnetosphere: a global MHD simulation. J. Geophys. Res. [Space Phys.], 117, A05225, 2012, DOI: 10.1029/2012JA017575.

Jia, X., J.A. Slavin, T.I. Gombosi, L.K.S. Daldorff, G. Toth, and B. van der Holst. Global MHD simulations of Mercury's magnetosphere with coupled planetary interior: induction effect of the planetary conducting core on the global interaction. J. Geophys. Res. [Space Phys.], 120, 4763-4775, 2015, DOI: 10.1002/2015JA021143.

Johnson, R.E. Plasma-induced sputtering of an atmosphere. Space Sci. Rev., 69, 215-253, 1994.

Johnson, R.E. Polar caps on Ganymede and Io revisited. Icarus, 128, 469-471, 1997, DOI: 10.1006/icar.1997.5746.

Johnson, R.E. Surface chemistry in the Jovian magnetosphere radiation environment. In: R. Dessler, Editor. Chemical Dynamics in Extreme Environments, Adv. Ser. Phys. Chem. World Scientific, Singapore 11, 390-419 (Chapter 8), 2001.

Johnson, R.E., R.W. Carlson, J.F. Cooper, C. Paranicas, M.H. Moore, and M.C. Wong. Radiation effects on the surface of the Galilean satellites. In: F. Bagenal, T. Dowling, and W. McKinnon, Editors. Jupiter: The Planet, Satellites and Magnetosphere, Cambridge Univ. Press, 485-512, (Chapter 20), 2004.

Johnson, R.E., J.G. Luhmann, R.L. Tokar, M. Bouhram, J.J. Berthelier, et al. Production ionization and redistribution of $\mathrm{O}_{2}$ in Saturn's ring atmosphere. Icarus, 180, 393-402, 2006, DOI: 10.1016/j.icarus.2005.08.021.

Johnson, R.E., M.H. Burger, T.A. Cassidy, F. Leblanc, M. Marconi, and W.H. Smyth. Composition and Detection of Europa's SputterInduced Atmosphere. In: R.T. Pappalardo, W.B. McKinnon, K. Khurana, and K. Khurana, Editors. Europa, University of Arizona Press, Tucson, 507-527, 2009.

Kabin, K., T.I. Gombosi, D.L. Dezeeuw, and K.G. Powell. Interaction of Mercury with the solar wind. Icarus, 143 (2), 397, 2000.

Kahler, S.W., N.R. Sheeley Jr., R.A. Howard, D.J. Michels, M.J. Koomen, R.E. McGuire, T.T. von Rosenvinge, and D.V. Reames. Associations between coronal mass ejections and solar energetic proton events. J. Geophys. Res., 89, 9683-9693, 1984, DOI: 10.1029/JA089iA11p09683.

Kallio, E., and P. Janhunen. Solar wind and magnetospheric ion impact on Mercury's surface. Geophys. Res. Lett., 30 (17), 1877, 2003, DOI: 10.1029/2003GL017842.

Kallio, E., and P. Janhunen. The response of the Hermean magnetosphere to the interplanetary magnetic field. Adv. Space Res., 33 (12), 2176-2181, 2004

Kameda, S., I. Yoshikawa, M. Kagitani, and S. Okano. Interplanetary dust distribution and temporal variability of Mercury's atmospheric Na. Geophys. Res. Lett., 36, L15201, 2009, DOI: $10.1029 / 2009$ GL039036.

Keating, G.M., J.L. Bertaux, S.W. Bougher, R.E. Dickinson, T.E. Cravens, et al. Models of Venus neutral upper atmosphere: Structure and composition. Adv. Space Res., 5 (11), 117-171, 1985, DOI: 10.1016/0273-1177(85)90200-5.

Khurana, K.K., M.G. Kivelson, D.J. Stevenson, G. Schubert, C.T. Russell, R.J. Walker, and C. Polanskey. Induced magnetic fields as evidence for subsurface oceans in Europa and Callisto. Nature, 395, 777-780, 1998, DOI: 10.1038/27394.

Khurana, K.K., M.G. Kivelson, V.M. Vasyliunas, N. Krupp, J. Woch, A. Lagg, B.H. Mauk, and W.S. Kurth. The configuration of Jupiter's magnetosphere. In: F. Bagenal, T. Dowlingand, and W. McKinnon, Editors. Jupiter: The Planet, Satellites, Magnetosphere, Cambridge University Press, UK, 593-616, 2004.

Khurana, K.K., R.T. Pappalardo, N. Murphy, and T. Denk. The origin of Ganymede's polar caps. Icarus, 191, 193-202, 2007.

Khurana, K.K., C.T. Russell, and M.K. Dougherty. Magnetic portraits of Tethys and Rhea. Icarus, 193, 465-474, 2008, DOI: $10.1016 /$ j.icarus.2007.08.005.

Khurana, K.K., M.G. Kivelson, K.P. Hand, and C.T. Russel. Electromagnetic induction from Europa's ocean and the deep interior. In: Robert.T. Pappalardo, William.B. McKinnon, and K. Khurana, Editors. Europa, University of Arizona Press, Tucson, 572-586, 2009.

Killen, R., G. Cremonese, H. Lammer, S. Orsini, A.E. Potter, et al. Processes that Promote and Deplete the Exosphere of Mercury. Space Sci. Rev., 132 (2-4), 433-509, 2007, DOI: $10.1007 / \mathrm{s} 11214-007-9232-0$.

Killen, R.M., and W.-H. Ip. The surface-bounded atmospheres of Mercury and the Moon. Rev. Geophys., 37 (i.3), 361-406, 1999.

Killen, R.M., A.E. Potter, P. Reiff, M. Sarantos, B.V. Jackson, P. Hick, and B. Giles. Evidence of space weather at Mercury. J. Geophys. Res., 106 (E9), 20509-20525, 2001.

Killen, R.M., T.A. Bida, and T.H. Morgan. The calcium exosphere of Mercury. Icarus, 173 (2), 300-311, 2005.

Killen, R.M., and J.M. Hahn. Impact vaporization as a possible source of Mercury's calcium exosphere. Icarus, 250, 230-237, 2015

Kim, M.-H.Y., F.A. Cucinotta, H.N. Nounu, C. Zeitlin, D.M. Hassler, et al. Comparison of Martian surface ionizing radiation measurements from MSL-RAD with Badhwar-O'Neill 2011/ HZETRN model calculations. J. Geophys. Res. [Planets], 119, 1311-1321, 2014, DOI: 10.1002/2013JE004549.

Kivelson, M.G., and C.T. Russell. Introduction to space physics, Cambridge University Press, Cambridge, ISBN: 0-521-45104-3, 1995.

Kivelson, M.G., K.K. Khurana, C.T. Russell, R.J. Walker, J. Warnecke, F.V. Coroniti, C. Polanskey, D.J. Southwood, and G. Schubert. Discovery of Ganymede's magnetic field by the Galileo spacecraft. Nature, 384, 537-541, 1996.

Kivelson, M.G., K.K. Khurana, F.V. Coroniti, S. Joy, C.T. Russell, R.J. Walker, J. Warnecke, L. Bennett, and C. Polanskey. Magnetic field and magnetosphere of Ganymede. Geophys. Res. Lett., 24, 2155, 1997, DOI: 10.1029/97GL02201.

Kivelson, M.G., F. Bagenal, W.S. Kurth, F.M. Neubauer, C. Paranicas, and J. Saur. Magnetospheric interactions with satellites. In: F. Bagenal, T. Dowling, and W. McKinnon, Editors. Jupiter: The Planet, Satellites and Magnetosphere, Cambridge University Press, UK, 513-536, 2004.

Kivelson, M.G., and F. Bagenal. Planetary Magnetospheres. In: Encyclopedia of the Solar System, Academic Press-Elsevier, ISBN-13: 978-0-12-088589-3/ISBN-10: 0-12-088589-1, (Chapter 28), 2007.

Kminek, G., and J.L. Bada. The effect of ionizing radiation on the preservation of amino acids on Mars. Earth Planet. Sci. Lett., 245, 1-5, 2006, DOI: 10.1016/j.eps1.2006.03.008.

Kollmann, P., E. Roussos, C. Paranicas, N. Krupp, and D.K. Haggerty. Processes forming and sustaining Saturn's proton radiation belts. Icarus, 222, 323-341, 2013, DOI: $10.1016 /$ j.icarus.2012.10.033.

Kotova, A., E. Roussos, N. Krupp, and I. Dandouras. Simulation of the galactic cosmic rays interaction with Saturn's atmosphere and rings, in: COSPAR communication, Moscow, 2014.

Kotova, A., E. Roussos, N. Krupp, and I. Dandouras. Modeling of the energetic ion observations in the vicinity of Rhea and Dione. Icarus, 258, 402-417, 2015, DOI: 10.1016/j.icarus.2015.06.031. 
Kriegel, H., S. Simon, J. Müller, U. Motschmann, J. Saur, K.-H. Glassmeier, and M.K. Dougherty. The plasma interaction of Enceladus: 3D hybrid simulations and comparison with Cassini MAG data. Planet. Space Sci., 57, 2113-2122, 2009,

DOI: 10.1016/j.pss.2009.09.025.

Krimigis, S.M., C.O. Bostrom, A.F. Cheng, T.P. Armstrong, and W.I. Axford. Hot plasma and energetic particles in Neptune's magnetosphere. Science, 246, 1483-1489, 1989,

DOI: $10.1126 /$ science.246.4936.1483.

Krimigis, S.M., D.G. Mitchell, D.C. Hamilton, N. Krupp, S. Livi, et al. Dynamics of Saturn's magnetosphere from MIMI during Cassini's orbital insertion. Science, 307, 1270-1273, 2005, DOI: $10.1126 /$ science.1105978.

Krimigis, S.M., N. Sergis, D.G. Mitchell, D.C. Hamilton, and N. Krupp. A dynamic, rotating ring current around Saturn. Nature, 450, 1050-1053, 2007, DOI: 10.1038/nature06425.

Krimigis, S.M., D.G. Mitchell, E.C. Roelof, K.C. Hsieh, and D.J. McComas. Imaging the interaction of the heliosphere with the interstellar medium from Saturn with Cassini. Science, 326 (5955), 971, 2009.

Kronberg, E.A., J. Woch, N. Krupp, A. Lagg, K.K. Khurana, and K.-H. Glassmeier. Mass release at Jupiter: substorm-like processes in the Jovian magnetotail. J. Geophys. Res., 110, A03211, 2005, DOI: 10.1029/2004JA010777.

Krupp, N. Comparison of plasma sources in Solar System magnetospheres. Space Sci. Rev., 192, 285-295, 2015, DOI: $10.1007 / \mathrm{s} 11214-015-0176-5$.

Ksanfomaliti, L.V., N.M. Vasilchikov, O.F. Ganpantserova, E.V. Petrova, A.P. Suvorov, G.F. Filippov, O.V. Iablonskaia, and L.V. Iabrova. Electrical discharges in the atmosphere of Venus. Sov. Astron. Lett., 5, 122-126, 1979.

Laakso, H., D.H. Fairfield, M.R. Collier, H. Opgenoorth, T.-D. Phan, et al. Oscillations of magnetospheric boundaries driven by IMF rotations. Geophys. Res. Lett., 25 (15), 3007-3010, 1998.

Lai, H.R., H.Y. Wei, C.T. Russell, C.S. Arridge, and M.K. Dougherty. Reconnection at the magnetopause of Saturn: perspective from FTE occurrence and magnetosphere size. J. Geophys. Res. [Space Phys.], 117, A05222, 2012, DOI: 10.1029/2011JA017263.

Lamy, L., P. Schippers, P. Zarka, B. Cecconi, C.S. Arridge, et al. Properties of Saturn kilometric radiation measured within its source region. Geophys. Res. Lett., 37, L12104, 2010, DOI: 10.1029/2010GL043415.

Lamy, L., B. Cecconi, P. Zarka, P. Canu, P. Schippers, et al. Emission and propagation of Saturn kilometric radiation: magnetoionic modes, beaming pattern, and polarization state. J. Geophys. Res. [Space Phys.], 116, A04212, 2011, DOI: 10.1029/2010JA016195.

Lamy, L., R. Prangé, K.C. Hansen, J.T. Clarke, P. Zarka, et al. Earthbased detection of Uranus' aurorae. Geophys. Res. Lett., 39, L07105, 2012, DOI: 10.1029/2012GL051312.

Landgraf, M., J.-C. Liou, H.A. Zook, and E. Grun. Origins of Solar System dust beyond Jupiter. Astroph. J., 123, 2857-2861, 2002.

Landis, G.A., S.G. Bailey, and R. Tischler. Causes of Power-Related Satellite Failures. In: IEEE 4th World conference on photovoltaic energy conversion, 2, 1943, 2006,

DOI: $10.1109 /$ WCPEC.2006.279878.

Larson, E.J.L., O.B. Toon, R.A. West, and A.J. Friedson. Microphysical modeling of Titan's detached haze layer in a 3D GCM. Icarus, 254, 122-134, 2015,

DOI: 10.1016/j.icarus.2015.03.010.

Laurenza, M., E.W. Cliver, J. Hewitt, M. Storini, A.G. Ling, C.C. Balch, and M.L. Kaiser. A technique for short-term warning of solar energetic particle events based on flare location, flare size, and evidence of particle escape. Space Weather, 7, S04008, 2009, DOI: $10.1029 / 2007$ SW000379.

Lavraud, B., E. Larroque, E. Budnik, V. Génot, J.E. Borovsky, et al. Asymmetry of magnetosheath flows and magnetopause shape during low Alfven Mach number solar wind. J. Geophys. Res., 118, 1089, 2013, DOI: 10.1002/jgra.50145.
Lavvas, P., R.A. West, G. Gronoff, and P. Rannou. Titan's emission processes during eclipse. Icarus, 241, 397-408, 2014.

Leblanc, F., and R.E. Johnson. Mercury's sodium exosphere. Icarus, 164, 261-281, 2003.

Leblanc, F., A.E. Potter, R.M. Killen, and R.E. Johnson. Origins of Europa Na cloud and torus. Icarus, 178, 367-385, 2005, DOI: $10.1016 /$ j.icarus.2005.03.027.

Leblanc, F., J.G. Luhmann, R.E. Johnson, and M. Liu. Solar Energetic particle event at Mercury. Planet. Space Sci., 51, 339-352, 2003

Leblanc, F., J.Y. Chaufray, J. Lilensten, O. Witasse, and J.L. Bertaux. Martian dayglow as seen by the SPICAM UV spectrograph on Mars Express. J. Geophys. Res. [Planets], 111, E09S11, 2006a, DOI: $10.1029 / 2005 J E 002664$.

Leblanc, F., O. Witasse, J. Winningham, D. Brain, J. Lilensten, P.L. Blelly, R.A. Frahm, J.S. Halekas, and J.L. Bertaux. Origins of the Martian aurora observed by Spectroscopy for investigation of characteristics of the atmosphere of Mars (SPICAM) on board Mars Express. J. Geophys. Res. [Space Phys.], 111, A09313, 2006b, DOI: 10.1029/2006JA011763.

Leblanc, F., A. Doressoundiram, N. Schneider, V. Mangano, A. López Ariste, C. Lemen, B. Gelly, C. Barbieri, and G. Cremonese. High latitude peaks in Mercury's sodium exosphere: spectral signature using THEMIS solar telescope. Geophys. Res. Lett., 35, L18204, 2008, DOI: 10.1029/2008GL035322.

Lilensten, J., and A. Belehaki. Developing the scientific basis for monitoring, modelling and predicting space weather. Acta. Geophys, 57, 1-14, 2009, DOI: 10.2478/s11600-008-0081-3.

Lilensten, J., C. Simon, O. Witasse, O. Dutuit, R. Thissen, and C. Alcarez. A fast comparison of the diurnal secondary ion production in the ionosphere of Titan. Icarus, 174, 285, $2005 \mathrm{a}$.

Lilensten, J., O. Witasse, C. Simon, H. Soldi-Lose, O. Dutuit, R. Thissen, and C. Alcaraz. Prediction of a $\mathrm{N}_{2}{ }^{++}$layer in the upper atmosphere of Titan. Geophys. Res. Lett., 32, L03203, 2005b, DOI: 10.1029/2004GL021432.

Lilensten, J., C. Simon Wedlund, M. Barthélémy, R. Thissen, D. Ehrenreich, G. Gronoff, and O. Witasse. Dications and thermal ions in planetary atmospheric escape. Icarus, 222, 169-187, 2013, DOI: 10.1016/j.icarus.2012.09.034.

Lilensten, J., A.J. Coates, V. Dehant, T. Dudok de Wit, R.B. Horne, F. Leblanc, J. Luhmann, E. Woodfield, and M. Barthélemy. What characterizes planetary space weather? Astron. Astrophys. Rev., 22-79, 2014, DOI: 10.1007/s00159-014-0079-6.

Lilensten, J., D. Bernard, M. Barthélémy, G. Gronoff, C. Simon Wedlund, and A. Opitz. The blue, red and green aurorae at the Red planet. Planet. Space Sci., 2015.

Lollo, A., P. Withers, K. Fallows, Z. Girazian, M. Matta, and P.C. Chamberlin. Numerical simulations of the ionosphere of Mars during a solar flare. J. Geophys. Res., 117 (A5), A05314, 2012.

Lopes, R.M.C., and D.A. Williams. Io after Galileo. Rep. Prog. Phys., 68, 303-340, 2005, DOI: 10.1088/0034-4885/68/2/R02.

López-Moreno, J.J., G.J. Molina-Cuberos, M. Hamelin, R. Grard, F. Simões, et al. Structure of Titan's low altitude ionized layer from the Relaxation Probe onboard HUYGENS. Geophys. Res. Lett., 35, L22104, 2008, DOI: 10.1029/2008GL035338.

Lorenzato, L., A. Sicard, and S. Bourdarie. A physical model for electron radiation belts of Saturn. J. Geophys. Res. [Space Phys.], 117, A08214, 2012, DOI: 10.1029/2012JA017560.

Louarn, P., A. Roux, S. Perraut, W. Kurth, and D. Gurnett. A study of the large-scale dynamics of the Jovian magnetosphere using the Galileo plasma wave experiment. Geophys. Res. Lett., 25, 2905-2908, 1998.

Lucchetti, A., C. Plainaki, G. Cremonese, A. Milillo, T. Cassidy, $\mathrm{X}$. Jia, and V. Shematovich. Loss rates of Europa's exosphere. Planet. Space Sci., 2016, in press,

DOI: 10.1016/j.pss.2016.01.009.

Luhmann, J.G., R.J. Warniers, C.T. Russell, J.R. Spreiter, and S.S. Stahara. A gas dynamic magnetosheath field model for unsteady interplanetary fields - Application to the solar wind interaction 
with Venus. J. Geophys. Res., 91, 3001-3010, 1986, DOI: 10.1029/JA091iA03p03001.

Luhmann, J.G., C.T. Russell, F.L. Scarf, L.H. Brace, and W.C. Knudsen. Characteristics of the marslike limit of the venus-solar wind interaction. J. Geophys. Res., 92, 8545-8557, 1987.

Luhmann, J.G., W.T. Kasprzak, and C.T. Russell. Space weather at Venus and its potential consequences for atmosphere evolution. J. Geophys. Res. [Planets], 112, E04S10, 2007, DOI: $10.1029 / 2006 J E 002820$.

Luhmann, J.G., A. Fedorov, S. Barabash, E. Carlsson, Y. Futaana, et al. Venus Express observations of atmospheric oxygen escape during the passage of several coronal mass éjections. J. Geophys. Res., 113 (52), E00B04, 2008, DOI: 10.1029/2008JE003092.

Lundin, R., A. Zakharov, R. Pellinen, S.W. Barabash, H. Borg, et al. ASPERA/Phobos measurements of the ion outflow from the Martian ionosphere. Geophys. Res. Lett., 17, 873-876, 1990.

Lundin, R., H. Lammer, and I. Ribas. Planetary magnetic fields and solar forcing: implications for atmospheric evolution. Space Sci. Rev., 129, 245-278, 2007.

Lundin, R., S. Barabash, A. Fedorov, M. Holmstrom, H. Nilsson, J. Sauvaud, and M. Yamauchi. Solar forcing and planetary ion escape from Mars. Geophys. Res. Let., 35, 9203, 2008.

Majeed, T., and J.C. McConnell. The upper ionospheres of Jupiter and Saturn. Planet. Space Sci., 39, 1715-1732, 1991.

Mandt, K.E., D.A. Gell, M. Perry, J.H. Waite Jr., F.A. Crary, et al. Ion densities and composition of Titan's upper atmosphere derived from the Cassini Ion Neutral Mass Spectrometer: analysis methods and comparison of measured ion densities to photochemical model simulations. J. Geophys. Res., 117, E10006, 2012, DOI: 10.1029/2012JE004139.

Mangano, V., S. Massetti, A. Milillo, C. Plainaki, S. Orsini, and F. Leblanc. THEMIS Na exosphere observations of Mercury and their correlation with in-situ magnetic field measurements by MESSENGER. Planet. Space Sci., 115, 102-109, 2015.

Marconi, M.L. A kinetic model of Ganymede's atmosphere. Icarus, 190, 155-174, 2007.

Massetti, S., S. Orsini, A. Milillo, A. Mura, E. de Angelis, H. Lammer, and P. Wurz. Mapping of the cusp plasma precipitation on the surface of Mercury. Icarus, 166 (i.2), 229-237, 2003.

Masters, A. Magnetic reconnection at Uranus' magnetopause. J. Geophys. Res. [Space Phys.], 119, 5520-5538, 2014, DOI: 10.1002/2014JA020077.

Masters, A., S.J. Schwartz, E.M. Henley, M.F. Thomsen, B. Zieger, et al. Electron heating at Saturn's bow shock. J. Geophys. Res. [Space Phys.], 116, A10107, 2011.

Masters, A., N. Achilleos, J.C. Cutler, A.J. Coates, M.K. Dougherty, and G.H. Jones. Surface waves on Saturn's magnetopause. Planet. Space Sci., 65, 109-121, 2012, DOI: $10.1016 /$ j.pss.2012.02.007.

Masunaga, K., Y. Futaana, G. Stenberg, S. Barabash, T.L. Zhang, A. Fedorov, S. Okano, and N. Terada. Dependence of $\mathrm{O}^{+}$escape rate from the Venusian upper atmosphere on IMF directions. Geophys. Res. Lett., 40 (9), 1682-1685, 2014, DOI: $10.1002 /$ grl.50392.

Mauk, B.H. Comparative investigation of the energetic ion spectra comprising the magnetospheric ring currents of the Solar System. J. Geophys. Res. [Space Phys.], 119 (12), 9729-9746, 2014.

Mauk, B.H., and N.J. Fox. Electron radiation belts of the Solar System. J. Geophys. Res., 115, A12220, 2010, DOI: $10.1029 / 2010 J A 015660$.

Mauk, B.H., S.M. Krimigis, E.P. Keath, A.F. Cheng, and T.P. Armstrong. The hot plasma and radiation environment of the Uranian magnetosphere. J. Geophys. Res., 92, 15283-15308, 1987, DOI: 10.1029/ JA092iA13p15283.

Mauk, B.H., E.P. Keath, M. Kane, M. Krimigis, A.F. Cheng, M.H. Acuña, T.P. Armstrong, and N.F. Ness. The magnetosphere of Neptune - hot plasmas and energetic particles. J. Geophys. Res. [Space Phys.], 96, 19061, 1991.
Mauk, B.H., S.M. Krimigis, A.F. Cheng, and R.S. Selesnick. Energetic particles and hot plasmas of Neptune. In: D.P. Cruikshank, M.S. Matthews, and A.M. Schumann, Editors. Neptune and Triton, Astronomisches Rechen-Institut Publisher, Germany, 169-232, 1995.

Mauk, B.H., S.A. Gary, M. Kane, E.P. Keath, S.M. Krimigis, and T.P. Armstrong. Hot plasma parameters of Jupiter's inner magnetosphere. J. Geophys. Res. [Space Phys.], 101, 7685-7696, 1996, DOI: 10.1029/96JA00006.

Mauk, B.H., D.G. Mitchell, S.M. Krimigis, E.C. Roelof, and C.P. Paranicas. Energetic neutral atoms from a trans-Europa gas torus at Jupiter. Nature, 421, 920-922, 2003, DOI: $10.1038 /$ nature 01431 .

Mauk, B.H., D.C. Hamilton, T.W. Hill, G.B. Hospodarsky, R.E. Johnson, et al. Fundamental plasma processes in Saturn's magnetosphere. In: M.K. Dougherty, L.W. Esposito, and S.M. Krimigis, Editors. Saturn from CassiniHuygens, Springer Science+Business Media B.V, Heidelberg, 281, 2009.

Mavromichalaki, H., A. Papaioannou, C. Plainaki, C. Sarlanis, G. Souvatzoglou, et al. Applications and usage of the real-time Neutron Monitor Database. Adv. Space Res., 47 (12), 2210-2222, 2011, DOI: 10.1016/j.asr.2010.02.019.

Mays, M.L., N.P. Savani, G. Collinson, A. Taktakishvili, P.J. MacNeice, and Y. Zheng. Propagation of the 2014 January 7 $\mathrm{CME}$ and resulting geomagnetic non-event. Astrophys. J., 812, 145,2015

McAndrews, H.J., C.J. Owen, M.F. Thomsen, B. Lavraud, A.J. Coates, M.K. Dougherty, and D.T. Young. Evidence for reconnection at Saturn's magnetopause. J. Geophys. Res. [Space Phys.], 113, A04210, 2008, DOI: 10.1029/2007JA012581.

McClintock, W.E., and M.R. Lankton. The Mercury atmospheric and surface composition spectrometer for the MESSENGER mission. Space Sci. Rev., 131 (1-4), 481-521, 2007.

McComas, D.J., F. Allegrini, P. Bochsler, M. Bzowski, E.R. Christian, et al. Global observations of the interstellar interaction from the Interstellar Boundary Explorer (IBEX). Science, 326 (5955), 959, 2009.

McEwen, A.S. Exogenic and endogenic albedo and color patterns on Europa. J. Geophys. Res., 91, 8077-8097, 1986.

McGrath, M.A., E. Lellouch, D.F. Strobel, P.D. Feldman, and R.E. Johnson. Satellite atmospheres. In: F. Bagenal, T. Dowling, and W. McKinnon, Editors. Jupiter: The Planet, Satellites and Magnetosphere, Cambridge University Press, UK, 457-483, 2004.

McGrath, M.A., X. Jia, K. Retherford, P.D. Feldman, D.F. Strobel, and J. Saur. Aurora on Ganymede. J. Geophys. Res. [Space Phys.], 118, 2043-2054, 2013, DOI: 10.1002/jgra.50122.

McKinnon, W.B., and R.L. Kirk. In: L.-A. McFadden, P.R. Weissman, and T.V. Weissman, Editors. Encyclopedia of the Solar System, Academic Press, Elsevier Ed., UK, 483-502, ISBN: 978-0-12-088589-3, Chapter 26, 2007.

McNutt, R.L., J.W. Belcher, and H.S. Bridge. Positive ion observations in the middle magnetosphere of Jupiter. J. Geophys. Res. [Space Phys.], 86, 8319-8342, 1981, DOI: $10.1029 / \mathrm{JA0} 086 \mathrm{AA} 10 \mathrm{p} 08319$.

Melin, H., D.E. Shemansky, and X. Liu. The distribution of atomic hydrogen and oxygen in the magnetosphere of Saturn. Planet. Space Sci., 57, 1743-1753, 2009, DOI: $10.1016 /$ j.pss.2009.04.014.

Meredith, C.J., S.W.H. Cowley, and J.D. Nichols. Survey of Saturn auroral storms observed by the Hubble Space Telescope: implications for storm time scales. J. Geophys. Res. [Space Phys. J, 119, 9624-9642, 2014, DOI: 10.1002/2014JA020601.

Merka, J., A. Szabo, J. Šafránková, and Z. Němeček. Earth's bow shock and magnetopause in the case of a field-aligned upstream flow: Observation and model comparison. J. Geophys. Res. [Space Phys.], 108 (A7), SMP 2-1, 2007,

DOI: $10.1029 / 2002 J A 009697$. 
Michael, M., S.N. Tripathi, W.J. Borucki, and R.C. Whitten. Highly charged cloud particles in the atmosphere of Venus. J. Geophys. Res., 114, E04008, 2009, DOI: 10.1029/2008JE003258.

Milan, S.E., B. Hubert, and A. Grocott. Formation and motion of a transpolar arc in response to dayside and nightside reconnection. J. Geophys. Res. [Space Phys.], 110, A01212, 2005, DOI: $10.1029 / 2004 J A 010835$.

Mileikowsky, C., F.A. Cucinotta, J.W. Wilson, B. Gladman, G. Horneck, et al. Natural transfer of viable microbes in space. Icarus, 145, 391-427, 2000, DOI: 10.1006/icar.1999.6317.

Milillo, A., P. Wurz, S. Orsini, D. Delcourt, E.Kallio, et al. Surfaceexosphere-magnetosphere system of Mercury. Space Sci. Rev., 117 (3-4), 397-443, 2005.

Milillo, A., M. Fujimoto, E. Kallio, S. Kameda, F. Leblanc, et al. The BepiColombo mission: an outstanding tool for investigating the Hermean environment. Planet. Space Sci., 58 (1), 40-60, 2010, DOI: 10.1016/j.pss.2008.06.005.

Milillo, A., C. Plainaki, E. De Angelis, V. Mangano, S. Massetti, A. Mura, S. Orsini, and R. Rispoli. Analytical model of Europa's $\mathrm{O}_{2}$ exosphere. Planet. Space Sci., 2015, in press, DOI: 10.1016/j.pss.2015.10.011

Miller, S., A. Aylward, and G. Millward. Giant planet ionospheres and thermospheres: the importance of ion-neutral coupling. Space Sci. Rev., 116 (1), 319-343, 2005.

Mitchell, D.G., F. Kutchko, D.J. Williams, T.E. Eastman, L.A. Frank, and C.T. Russell. An extended study of the low-latitude boundary layer on the dawn and dusk flanks of the magnetosphere. J. Geophys. Res. [Space Phys.], 92 (A7), 7394-7404, 1987.

Mitchell, D.G., S.M. Krimigis, C. Paranicas, P.C. Brandt, J.F. Carbary, et al. Recurrent energization of plasma in the midnightto-dawn quadrant of Saturn's magnetosphere, and its relationship to auroral UV and radio emissions. Planet. Space Sci., 57, 1732-1742, 2009, DOI: 10.1016/j.pss.2009.04.002.

Miyasaka, H., K. Nagata, T. Doke, J. Kikuchi, K. Maezawa, et al. Solar energetic particles events observed with EIS onboard NOZOMI spacecraft. 28th International Cosmic Ray Conference, 1, 3265, 2003

Miyoshi, Y., H. Misawa, A. Morioka, T. Kondo, Y. Koyama, and J. Nakajima. Observation of short-term variation of Jupiter's synchrotron radiation. Geophys. Res. Lett., 26, 9-12, 1999 , DOI: $10.1029 / 1998 G L 900244$

Molina-Cuberos, G. Cosmic ray and UV radiation models on the ancient Martian surface. Icarus, 154, 216-222, 2001, DOI: 10.1006/icar.2001.6658

Molina-Cuberos, G.J., J.J. López-Moreno, R. Rodrigo, $\mathrm{H}$. Lichtenegger, and K. Schwingenschuh. A model of the Martian ionosphere below $70 \mathrm{~km}$. Adv. Space Res., 27, 1801-1806, 2001, DOI: 10.1016/S0273-1177(01)00342-8.

Montmessin, F., J.-L. Bertaux, F. Lefèvre, E. Marcq, D. Belyaev, et al. A layer of ozone detected in the nightside upper atmosphere of Venus. Icarus, 216 (1), 82-85, 2011, DOI: 10.1016/j.icarus.2011.08.010.

Moore, M.H. Studies of proton-irradiated $\mathrm{SO}_{2}$ at low temperatures implications for Io. Icarus, 59, 114-128, 1984, DOI: 10.1016/0019-1035(84)90059-9.

Moses, J.I., B. Bézard, E. Lellouch, G.R. Gladstone, H. Feuchtgruber, and M. Allen. Photochemistry of Saturn's atmosphere. I. Hydrocarbon chemistry and comparisons with ISO observations. Icarus, 143 (2), 244-298, 2000

Mura, A., S. Orsini, A. Milillo, A.M. Di Lellis, and E. De Angelis. Neutral atom imaging at Mercury. Planet. Space Sci., 54, 144-152, 2006.

Mura, A., P. Wurz, H.I.M. Lichtenegger, H. Schleicher, H. Lammer, et al. The sodium exosphere of Mercury: comparison between observations during Mercury's transit and model results. Icarus, 200 (1), 1-11, 2009.

Müller-Wodarg, I.C.F., L. Moore, M. Galand, S. Miller, and M. Mendillo. Magnetosphere-atmosphere coupling at Saturn: 1 Response of thermosphere and ionosphere to steady state polar forcing. Icarus, 221 (2), 481-494, 2012,

DOI: 10.1016/j.icarus.2012.08.034.

Nagy, A.F., A.J. Kliore, M. Mendillo, S. Miller, L. Moore, J.I. Moses, I. Müller-Wodarg, D. Shemansky, and M.K. Dougherty. Upper Atmosphere and Ionosphere of Saturn. In: M.K. Dougherty, et al., Editors. Saturn from Cassini-Huygens, Springer Science + Business Media B.V., Germany, 2009, DOI: 10.1007/978-1-4020-9217-6 8.

Ness, N.F., M.H. Acuña, L.F. Burlaga, J.E.P. Connerney, and R.P. Lepping. Magnetic fields at Neptune. Science, 233, 85-89, 1986.

Ness, N., M.H. Acuña, L.F. Burlaga, J.E.P. Connerney, R.P. Lepping, and F.N. Neubauer. Magnetic fields at Neptune. Science, 246 (4936), 1473-1478, 1989.

Nichols, J.D., S.W.H. Cowley, and D.J. McComas. Magnetopause reconnection rate estimates for Jupiter's magnetosphere based on interplanetary measurements at 5AU. Ann. Geophys., 24 (1), 393-406, 2006, DOI: 10.5194/angeo-24-393-2006.

Nichols, J.D., E.J. Bunce, J.T. Clarke, S.W.H. Cowley, J.-C. Gérard, D. Grodent, and W.R. Pryor. Response of Jupiter's UV auroras to interplanetary conditions as observed by the Hubble Space Telescope during the Cassini flyby campaign. J. Geophys. Res. [Space Phys.], 112, A02203, 2007, DOI: $10.1029 / 2006 J A 012005$.

Nichols, J.D., J.T. Clarke, S.W.H. Cowley, J. Duval, A.J. Farmer, J.C. Gérard, D. Grodent, and S. Wannawichian. Oscillation of Saturn's southern auroral oval. J. Geophys. Res. [Space Phys.], 113, A11205, 2008, DOI: 10.1029/2008JA013444.

Nichols, J.D., J.T. Clarke, J.C. Gerard, and D. Grodent. Observations of Jovian polar auroral filaments. Geophys. Res. Lett., 36, L08101, 2009a, DOI: 10.1029/2009GL037578.

Nichols, J.D., J.T. Clarke, J.C. Gérard, D. Grodent, and K.C. Hansen. Variation of different components of Jupiter's auroral emission. J. Geophys. Res. [Space Phys.], 114, A06210, 2009b,

DOI: 10.1029/2009JA014051

Nichols, J.D., B. Cecconi, J.T. Clarke, S.W.H. Cowley, J.C. Gérard, A. Grocott, D. Grodent, L. Lamy, and P. Zarka. Variation of Saturn's UV aurora with SKR phase. Geophys. Res. Lett., 37, L15102, 2010a, DOI: 10.1029/2010GL044057.

Nichols, J.D., S.W.H. Cowley, and L. Lamy. Dawn-dusk oscillation of Saturn's conjugate auroral ovals. Geophys. Res. Lett., 37, L24102, 2010b, DOI: 10.1029/2010GL045818.

Nichols, J.D., M.R. Burleigh, S.L. Casewell, S.W. Cowley, G.A. Wynn, J.T. Clarke, and A.A. West. Origin of electron cyclotron maser induced radio emissions at ultracool dwarfs: magnetosphere-ionosphere coupling currents. Astrophys. J., 760 (1), 59, 2012.

Nichols, J.D., S.V. Badman, K.H. Baines, R.H. Brown, E.J. Bunce, et al. Dynamic auroral storms on Saturn as observed by the Hubble Space Telescope. Geophys. Res. Lett., 41, 3323-3330, 2014, DOI: $10.1002 / 2014 G L 060186$.

Nilsson, H., N.J.T. Edberg, G. Stenberg, S. Barabash, M. Holmstrom, Y. Futaana, R. Lundin, and A. Fedorov. Heavy ion escape from Mars, influence from solar wind conditions and crustal magnetic fields. Icarus, 215, 475-484, 2011

Nishida, A. Reconnection in the Jovian magnetosphere. Geophys. Res. Lett., 10, 451-454, 1983.

Nordheim, T.A., L.R. Dartnell, L. Desorgher, A.J. Coates, and G.H. Jones. Ionization of the Venusian atmosphere from solar and galactic cosmic rays. Icarus, 245, 80-86, 2015, DOI: 10.1016/j.icarus.2014.09.032.

Norman, R.B., G. Gronoff, and C.J. Mertens. Influence of dust loading on atmospheric ionizing radiation on Mars. J. Geophys. Res. [Space Phys.], 119 (1), 452-461, 2014, DOI: $10.1002 / 2013 J A 019351$

Ockert-Bell, M.E., J.A. Burns, I.J. Daubar, P.C. Thomas, J. Veverka, M.J.S. Belton, and K.P. Klaasen. The structure of Jupiter's ring system as revealed by the Galileo imaging experiment. Icarus, 138, 188-213, 1999, DOI: 10.1006/icar.1998.6072.

Odstrčil, D. Modeling 3-D solar wind structure. Adv. Space Res., 32 (4), 497-506, 2003. 
Odstrčil, D., and V.J. Pizzo. Three-dimensional propagation of coronal mass ejections (CMEs) in a structured solar wind flow: 1. CME launched within the streamer belt. J. Geophys. Res. [Space Phys.], 104 (A1), 483-492, 1999a.

Odstrčil, D., and V.J. Pizzo. Distortion of the interplanetary magnetic field by three-dimensional propagation of coronal mass ejections in a structured solar wind. J. Geophys. Res. [Space Phys.], 104, 28225-28239, 1999b.

Odstrčil, D., M. Dryer, and Z. Smith. Propagation of an interplanetary shock along the heliospheric plasma sheet. J. Geophys. Res. [Space Phys.], 101 (A9), 19973-19986, 1996.

Odstrčil, D., P. Riley, and X.P. Zhao. Numerical simulation of the 12 May 1997 interplanetary CME event. J. Geophys. Res. [Space Phys.], 109, A02116, 2004, DOI: 10.1029/2003JA010135.

Ogilvie, K.W., and M.D. Desch. The wind spacecraft and its early scientific results. Adv. Space Res., 20 (4-5), 559-568, 1977

Orsini, S., S. Livi, K. Torkar, S. Barabash, A. Milillo, P. Wurz, A.M. Di Lellis, E. Kallio, and The SERENA team. SERENA: a suite of four instruments (ELENA, STROFIO, PICAM and MIPA) on board BepiColombo-MPO for particle detection in the Hermean environment. Planet. Space Sci., 58, 166-181, 2010.

Orsini, S., V. Mangano, A. Mura, D. Turrini, S. Massetti, A. Milillo, and C. Plainaki. The influence of space environment on the evolution of Mercury. Icarus, 239, 281-290, 2014.

Paranicas, C., W.R. Paterson, A.F. Cheng, B.H. Mauk, R.W. McEntire, L.A. Frank, and D.J. Williams. Energetic particle observations near Ganymede. J. Geophys. Res. [Space Phys.], 104, 17459-17470, 1999, DOI: 10.1029/1999JA900199.

Parker, T.J. Channels and Valley networks associated with Argyre Planitia, Mars. Lunar and Planetary Institute Science Conference Abstracts, 20, 826, 1989

Parker, T.J., D.S. Gorsline, R.S. Saunders, D.C. Pieri, and D.M. Schneeberger. Coastal geomorphology of the Martian northern plains. J. Geophys. Res., 98, 11061, 1993.

Paschmann, G., S. Haaland, and R. Treumann. Auroral plasma physics. Space Sci. Rev., 103, 1-4, 2002,

DOI: $10.1023 / \mathrm{A}: 1023030716698$.

Pätzold, M., B. Häusler, M.K. Bird, S. Tellmann, R. Mattei, et al. The structure of Venus' middle atmosphere and ionosphere. Nature, 450, 657-660, 2007.

Pätzold, M., F.M. Neubauer, L. Carone, A. Hagermann, C. Stanzel, et al. MaRS: Mars express radio science experiment. In: K. Fletcher, Editor. Mars Express, ESA Communication Production Office, Noordwijk, The Netherlands, 217-245, 2009.

Pavlov, A.K., A.V. Blinov, and A.N. Konstantinov. Sterilization of Martian surface by cosmic radiation. Planet. Space Sci., 50, 669-673, 2002, DOI: 10.1016/S0032-0633(01)00113-1.

Pavlov, A.A., G. Vasilyev, V.M. Ostryakov, A.K. Pavlov, and P. Mahaffy. Degradation of the organic molecules in the shallow subsurface of Mars due to irradiation by cosmic rays. Geophys. Res. Lett., 39, L13203, 2012, DOI: 10.1029/2012GL052166.

Perry, M.E., B. Teolis, H.T. Smith, R.L. McNutt, G. Fletcher, W. Kasprzak, B. Magee, D.G. Mitchell, and J.H. Waite. Cassini INMS observations of neutral molecules in Saturn's E-ring. J. Geophys. Res. [Space Phys.], 115, A10206, 2010, DOI: $10.1029 / 2010 J A 015248$.

Peter, K., M. Pätzold, G. Molina-Cuberos, O. Witasse, F. GonzálezGalindo, et al. The dayside ionospheres of Mars and Venus: comparing a one-dimensional photochemical model with MaRS (Mars Express) and VeRa (Venus Express) observations. Icarus, 233, 66-82, 2014, DOI: 10.1016/j.icarus.2014.01.028.

Phillips, J.L., A.I.F. Stewart, and J.G. Luhmann. The Venus ultraviolet aurora: observations at $130.4 \mathrm{~nm}$. Geophys. Res. Lett., 13 (10), 1047-1050, 1986

Piccialli, A., F. Montmessin, D. Belyaev, A. Mahieux, A. Fedorova, et al. Thermal structure of Venus nightside upper atmosphere measured by stellar occultations with SPICAV/Venus Express. Planet. Space Sci., 113-114, 321-335, 2015,

DOI: $10.1016 /$ j.pss.2014.12.009.
Pinilla-Alonso, N., T.L. Roush, G.A. Marzo, D.P. Cruikshank, and C.M. Dalle Ore. Iapetus surface variability revealed from statistical clustering of a VIMS mosaic: the distribution of $\mathrm{CO}_{2}$. Icarus, 215, 75-82, 2011,

DOI: 10.1016/j.icarus.2011.07.004.

Plainaki, C., A. Belov, E. Eroshenko, H. Mavromichalaki, and V. Yanke. Modeling ground level enhancements: event of 20 January 2005. J. Geophys. Res., 112, 4102, 2007.

Plainaki, C., A. Milillo, A. Mura, S. Orsini, and T. Cassidy. Neutral particle release from Europa's surface. Icarus, 210, 385-395, 2010a, DOI: 10.1016/j.icarus.2010.06.041.

Plainaki, C., H. Mavromichalaki, A. Belov, E. Eroshenko, M. Andriopoulou, and V. Yanke. A new version of the Neutron monitor based anisotropic GLE model: application to GLE60. Sol. Phys., 264 (1), 239-254, 2010b, DOI: $10.1007 / \mathrm{s} 11207-010-9576-6$.

Plainaki, C., A. Milillo, A. Mura, S. Orsini, S. Massetti, and T. Cassidy. The role of sputtering and radiolysis in the generation of Europa exosphere. Icarus, 218 (2), 956-966, 2012, DOI: $10.1016 / j$.icarus.2012.01.0232012.

Plainaki, C., A. Milillo, A. Mura, J. Saur, S. Orsini, and S. Massetti. Exospheric $\mathrm{O}_{2}$ densities at Europa during different orbital phases. Planet. Space Sci., 88, 42-52, 2013.

Plainaki, C., H. Mavromichalaki, M. Laurenza, M. Gerontidou, A. Kanellakopoulos, and M. Storini. The ground-level enhancement of 2012 may 17: derivation of solar proton event properties through the application of the NMBANGLE PPOLA model. Astrophys. J., 785, 160, 2014 DOI: $10.1088 / 0004-637 \mathrm{X} / 785 / 2 / 160$.

Plainaki, C., A. Milillo, S. Massetti, A. Mura, X. Jia, S. Orsini, V. Mangano, E. De Angelis, and R. Rispoli. The $\mathrm{H}_{2} \mathrm{O}$ and $\mathrm{O}_{2}$ exospheres of Ganymede: the result of a complex interaction between the Jovian magnetospheric ions and the icy moon. Icarus, 245, 306-319, 2015, DOI: 10.1016/j.icarus.2014.09.018.

Plainaki, C., P. Paschalis, D. Grassi, H. Mavromichalaki, and M. Andriopoulou. Solar energetic particle interactions with the Venusian atmosphere. Ann. Geophys., 34, 595-608, 2016, DOI: 10.5194/angeo-34-595-2016.

Porco, C., D. DiNino, and F. Nimmo. How the geysers, tidal stresses, and thermal emission across the South polar terrain of Enceladus are related. Astrophys. J., 148, 45, 2014, DOI: $10.1088 / 0004-6256 / 148 / 3 / 45$.

Porco, C.C., E. Baker, J. Barbara, K. Beurle, A. Brahic, et al. Imaging of Titan from the Cassini spacecraft. Nature, 434 (7030), 159-168, 2005.

Potter, A.E., C.M. Anderson, R.M. Killen, and T.H. Morgan. Ratio of sodium to potassium in the Mercury exosphere. J. Geophys. Res., 107, 6, 2002, DOI: 10.1029/2000JE001493.

Pudovkin, M.I., B.P. Besser, and S.A. Zaitsev. Magnetopause standoff distance in dependence on the magnetosheath and solar wind parameters. Ann. Geophys., 16, 388-396, 1998.

Radioti, A., J.-C. Gérard, D. Grodent, B. Bonfond, N. Krupp, and J. Woch. Discontinuity in Jupiter's main auroral oval. J. Geophys. Res. [Space Phys.], 113, A01215, 2008a, DOI: $10.1029 / 2007$ JA012610.

Radioti, A., D. Grodent, J.-C. Gérard, B. Bonfond, and J.T. Clarke. Auroral polar dawn spots: signatures of internally driven reconnection processes at Jupiter's magnetotail. Geophys. Res. Lett., 35, L03104, 2008b, DOI: 10.1029/2007GL032460.

Radioti, A., D. Grodent, J.-C. Gérard, E. Roussos, C. Paranicas, et al. Transient auroral features at Saturn: signatures of energetic particle injections in the magnetosphere. J. Geophys. Res., 114, A03210, 2009a, DOI: 10.1029/2008JA013632.

Radioti, A., A.T. Tomás, D. Grodent, J.-C. Gérard, J. Gustin, B. Bonford, N. Krupp, J. Woch, and J.D. Menietti. Equatorward diffuse auroral emissions at Jupiter: simultaneous HST and Galileo observations. Geophys. Res. Lett., 36, L07101, 2009b, DOI: $10.1029 / 2009$ GL037857.

Radioti, A., D. Grodent, J.-C. Gérard, and B. Bonfond. Auroral signatures of flow bursts released during magnetotail 
reconnection at Jupiter. J. Geophys. Res. [Space Phys.], 115, A07214, 2010, DOI: 10.1029/2009JA014844.

Radioti, A., D. Grodent, J.-C. Gérard, M.F. Vogt, M. Lystrup, and B. Bonfond. Nightside reconnection at Jupiter: auroral and magnetic field observations from 26 July 1998. J. Geophys. Res. [Space Phys.], 116, A03221, 2011a, DOI: 10.1029/2010JA016200.

Radioti, A., D. Grodent, J.-C. Gérard, S.E. Milan, B. Bonfond, J. Gustin, and W. Pryor. Bifurcations of the main auroral ring at Saturn: ionospheric signatures of consecutive reconnection events at the magnetopause. J. Geophys. Res. [Space Phys.], 116, A11209, 2011b, DOI: 10.1029/2011JA016661.

Radioti, A., E. Roussos, D. Grodent, J.-C. Gérard, N. Krupp, D.G. Mitchell, J. Gustin, B. Bonfond, and W. Pryor. Signatures of magnetospheric injections in Saturn's aurora. J. Geophys. Res. [Space Phys.], 118, 1922-1933, 2013a,

DOI: 10.1002 /jgra.50161.

Radioti, A., D. Grodent, J.-C. Gérard, B. Bonfond, J. Gustin, W. Pryor, J.M. Jasinski, and C.S. Arridge. Auroral signatures of multiple magnetopause reconnection at Saturn. Geophys. Res. Lett., 40, 4498-4502, 2013b, DOI: 10.1002/grl.50889.

Radioti, A., D. Grodent, J.-C. Gérard, S.E. Milan, R.C. Fear, C.M. Jackman, B. Bonfond, and W. Pryor. Saturn's elusive nightside polar arc. Geophys. Res. Lett., 41, 6321-6328, 2014, DOI: 10.1002/2014GL061081.

Radioti, A., D. Grodent, X. Jia, J.C. Gerard, B. Bonfond, W. Pryor, J. Gustin, D.G. Mitchell, and C.M. Jackman. A multi-scale magnetotail reconnection event at Saturn and associated flows: Cassini/UVIS observations. Icarus, 263 (1), 75-82, 2016, DOI: 10.1016/j.icarus.2014.12.016.

Raines, J.M., D.J. Gershman, T.H. Zurbuchen, M. Sarantos, and J.A. Slavin. Distribution and compositional variations of plasma ions in Mercury's space environment: the first three Mercury years of MESSENGER observations. J. Geophys. Res., 118 (4), 1604-1619, 2013

Raines, J.M., D.J. Gershman, J.A. Slavin, T.H. Zurbuchen, H. Korth, B.J. Anderson, and S.C. Solomon. Structure and dynamics of Mercury's magnetospheric cusp: MESSENGER measurements of protons and planetary ions. J. Geophys. Res. [Space Phys.], 119, 6587-6602, 2014, DOI: 10.1002/2014JA020120.

Rairden, R.L., L.A. Frank, and J.D. Craven. Geocoronal imaging with Dynamics Explorer. J. Geophys. Res., 91 (A12), 13613-13630, 1986, DOI: 10.1029/JA091iA12p13613.

Reames, D.V. Particle acceleration at the Sun and in the heliosphere. Space Sci. Rev., 90, 413, 1999, DOI: 10.1023/A:1005105831781.

Richard, M.S., T.E. Cravens, C. Wylie, D. Webb, Q. Chediak, et al. An empirical approach to modeling ion production rates in Titan's ionosphere II: ion production rates on the nightside. J. Geophys. Res. [Space Phys.], 120, 1281-1298, 2015a, DOI: $10.1002 / 2014 J A 020343$.

Richard, M.S., T.E. Cravens, C. Wylie, D. Webb, Q. Chediak, et al. An empirical approach to modeling ion production rates in Titan's ionosphere I: ion production rates on the dayside and globally. J. Geophys. Res. [Space Phys.], 120, 1264-1280, 2015b, DOI: 10.1002/2013JA019706.

Richardson, J.D. The solar wind and its interaction with the interstellar medium. In: N. Gopalswamy, et al., Editors. Heliophysical Processes, Springer-Verlag Berlin Heidelberg, Germany, Astrophysics and Space Science, 83 Proceedings, 83, 2010, DOI: 10.1007/978-3-642-11341-3 6.

Richardson, J.D., J.W. Belcher, M. Zhang, and R.L. McNutt Jr. Lowenergy ions near Neptune. J. Geophys. Res. [Space Phys.], 96 (18), 993, 1991.

Richardson, J.D., C. Wang, and L.F. Burlaga. The solar wind in the outer heliosphere. Adv. Space Res., 34 (1), 150-156, 2004, DOI: 10.1016/j.asr.2003.03.066.

Richer, E., R. Modolo, G.M. Chanteur, S. Hess, and F. Leblanc. A global jybrid model for Mercury's interaction with the solar wind: case study of the dipole representation. J. Geophys. Res., 117, A10228, 2012.
Rostoker, G., S.-I. Akasofu, J. Foster, R.A. Greenwald, Y. Kamide, K. Kawasaki, A.T.Y. Lui, R.L. Mcpherron, and C.T. Russell. Magnetospheric substorms - definition and signatures. J. Geophys. Res., 85 (A4), 1663-1668, 1980.

Roth, L., J. Saur, K.D. Retherford, D.F. Strobel, P.D. Feldman, M.A. McGrath, and F. Nimmo. Transient water vapor at Europa's south pole. Science, 343, 171-174, 2014a, DOI: $10.1126 /$ science. 1247051 .

Roth, L., K.D. Retherford, J. Saur, D.F. Strobel, P.D. Feldman, M.A. McGrath, and F. Nimmo. Orbital apocenter is not a sufficient condition for HST/STIS detection of Europa's water vapor aurora. Proc. Nat. Acad. Sci. U.S.A., 111 (48), E5123-E5132, 2014b.

Roth, L., J. Saur, K.D. Retherford, D.F. Strobel, P.D. Feldman, M.A. McGrath, J.R. Spencer, A. Blöcker, and N. Ivchenko. Europa's far ultraviolet oxygen aurora from a comprehensive set of HST observations. J. Geophys. Res. [Space Phys.], 121, 2143-2170, 2016, DOI: 10.1002/2015JA022073.

Roussos, E., G.H. Jones, N. Krupp, C. Paranicas, D.G. Mitchell, et al. Electron microdiffusion in the Saturnian radiation belts: Cassini MIMI/LEMMS observations of energetic electron absorption by the icy moons. J. Geophys. Res. [Space Phys.], 112, 6214, 2007, DOI: 10.1029/2006JA012027.

Roussos, E., N. Krupp, T.P. Armstrong, C. Paranicas, D.G. Mitchell, et al. Discovery of a transient radiation belt at Saturn. Geophys. Res. Lett., 35, L22106, 2008, DOI: 10.1029/2008GL035767.

Roussos, E., N. Krupp, C. Paranicas, J.F. Carbary, P. Kollmann, S.M. Krimigis, and D.G. Mitchell. The variable extension of Saturn's electron radiation belts. Planet. Space Sci., 104, 3-17, 2014, DOI: $10.1016 /$ j.pss.2014.03.021.

Russell, C.T., and R.C. Elphic. ISEE observations of flux transfer events at the dayside magnetopause. Geophys. Res. Lett., 6, 33-36, 1979, DOI: 10.1029/GL006i001p00033.

Russell, C.T., and F.L. Scarf. Evidence for lightning on Venus. $A d v$. Space Res., 10, 125-136, 1990,

DOI: $10.1016 / 0273-1177(90) 90173-W$.

Russell, C.T., K.K. Khurana, M.G. Kivelson, and D.E. Huddleston. Substorms at Jupiter: Galileo observations of transient reconnection in the near tail. Adv. Space Sci., 261 (10), 1499-1504, 2000.

Russell, C.T., T.L. Zhang, M. Delva, W. Magnes, R.J. Strangeway, and H.Y. Wei. Lightning on Venus inferred from whistler-mode waves in the ionosphere. Nature, 450, 661-662, 2007, DOI: 10.1038 /nature 05930 .

Sack, N.J., R.E. Johnson, J.W. Boring, and R.A. Baragiola. The effect of magnetospheric ion bombardment on the reflectance of Europa's surface. Icarus, 100, 534-540, 1992.

Saganti, P.B., F.A. Cucinotta, J.W. Wilson, L.C. Simonsen, and C. Zeitlin. Radiation climate map for analyzing risks to astronauts on the mars surface from galactic cosmic rays. Space Sci. Rev., 110, 14-156, 2004.

Samsonov, A.A., Z. Němeček, J. Šafránková, and K. Jelínek. Why does the subsolar magnetopause move sunward for radial interplanetary magnetic field? J. Geophys. Res., 117, A05221, 2012.

Santos-Costa, D., and S.A. Bourdarie. Modeling the inner Jovian electron radiation belt including non-equatorial particles. Planet. Space Sci., 49, 303-312, 2001,

DOI: 10.1016/S0032-0633(00)00151-3.

Santos-Costa, D., R. Sault, S. Bourdarie, D. Boscher, S. Bolton, et al. Synchrotron emission images from three-dimensional modeling of the Jovian electron radiation belts. Adv. Space Res., 28, 915-918, 2001, DOI: 10.1016/S0273-1177(01)00527-0.

Santos-Costa, D., M. Blanc, S. Maurice, and J.S. Bolton. Modelling the electron and proton radiation belts of Saturn. Geophys. Res. Lett., 30, 2059, 2003, DOI: 10.1029/2003GL017972.

Sarantos, M., J.A. Slavin, M. Benna, S.A. Boardsen, R.M. Killen, D. Schriver, and P. Trávníček. Sodium-ion pickup observed above the magnetopause during MESSENGER's first Mercury 
flyby: constraints on neutral exospheric models. Geophys. Res. Lett., 36 (4), L04106, 2009.

Saur, J., D.F. Strobel, and F.M. Neubauer. Interaction of the Jovian magnetosphere with Europa: constraints on the neutral atmosphere. J. Geophys. Res. [Space Phys.], 103, 19947-19962, 1998, DOI: 10.1029/97JE03556.

Saur, J., N. Schilling, F.M. Neubauer, D.F. Strobel, S. Simon, M.K. Dougherty, C.T. Russell, and R.T. Pappalardo. Evidence for temporal variability of Enceladus' gas jets: modeling of Cassini observations. Geophys. Res. Lett., 35, L20105, 2008, DOI: $10.1029 / 2008$ GL035811.

Saur, J., P.D. Feldman, L. Roth, F. Nimmo, D.F. Strobel, et al. Hubble Space Telescope/advanced camera for surveys observations of Europa's atmospheric ultraviolet emission at eastern elongation. Astrophys. J., 738, 153, 2011, DOI: $10.1088 / 0004-637 \mathrm{X} / 738 / 2 / 153$

Saur, J., S. Duling, L. Roth, X. Jia, D.F. Strobel, et al. The search for a subsurface ocean in Ganymede with Hubble Space Telescope observations of its auroral ovals. J. Geophys. Res. [Space Phys.], 120, 1715-1737, 2015, DOI: 10.1002/2014JA020778.

Schardt, A.W., and C.K. Goertz. High-energy particles. In: A.J. Dessler, Editor. Physics of the Jovian magnetosphere, Cambridge University Press, A83-26611 10-91 Cambridge and New York, 157-196, 1983.

Schilling, N., F.M. Neubauer, and J. Saur. Time-varying interaction of Europa with the Jovian magnetosphere: constraints on the conductivity of Europa's subsurface ocean. Icarus, 192, 41-55, 2007.

Schippers, P., N. André, R.E. Johnson, M. Blanc, I. Dandouras, A.J. Coates, S.M. Krimigis, and D.T. Young. Identification of photoelectron energy peaks in Saturn's inner neutral torus. J. Geophys. Res., 114 (A12), A12212, 2009.

Schmidt, C.A., J.K. Wilson, J. Baumgardner, and M. Mendillo. Orbital effects on Mercury's escaping sodium exosphere. Icarus, 207 (1), 9-16, 2010, DOI: 10.1016/j.icarus.2009.10.017

Schrijver, C.J. On a transition from solar-like coronae to rotationdominated Jovian-like magnetospheres in ultracool mainsequence stars. Astrophys. J. Lett., 699 (2), L148-L152, 2009.

Schunk, R., and A. Nagy. Ionospheres: Physics, Plasma Physics, and Chemistry. In: A.J. Dessler, J.R. Houghton, and M.J. Rycroft, Editors. 2nd edn., Cambridge Univ. Press, Cambridge, 2009.

Scudder, J.D., E.C. Sittler, and H.S. Bridge. A survey of the plasma electron environment of Jupiter - a view from Voyager. J. Geophys. Res. [Space Phys.], 86, 8157-8179, 1981, DOI: $10.1029 / J A 086 i A 10 p 08157$.

Seki, K., N. Terada, M. Yagi, D.C. Delcourt, F. Leblanc, and T. Ogino. Effects of the surface conductivity and the IMF strength on the dynamics of planetary ions in Mercury's magnetosphere. J. Geophys. Res. [Space Phys.], 118 (6), 3233-3242, 2013.

Seki, K., A. Nagy, C.M. Jackman, F. Crary, D. Fontaine, et al. A review of general physical and chemical processes related to plasma sources and losses for Solar System magnetospheres. Space Sci. Rev., 192 (1-4), 27-89, 2015.

Selesnick, R.S. Micro-and macro-signatures of energetic charged particles in planetary magnetospheres. Adv. Space Res., 13 (10), 221-230, 1993.

Selesnick, R.S., and J.D. Richardson. Plasmasphere formation in arbitrarily oriented magnetospheres. Geophys. Res. Lett., 13, 624-627, 1986, DOI: 10.1029/GL013i007p00624.

Sheel, V., S.A. Haider, P. Withers, K. Kozarev, I. Jun, S. Kang, G. Gronoff, and C. Simon Wedlund. Numerical simulation of the effects of a solar energetic particle event on the ionosphere of Mars. J. Geophys. Res., 117, A05312, 2012, DOI: $10.1029 / 2011 \mathrm{JA} 017455$

Shemansky, D.E., X. Liu, and H. Melin. The Saturn hydrogen plume. Planet. Space Sci., 57, 1659-1670, 2009,

DOI: $10.1016 /$ j.pss.2009.05.002.
Shematovich, V.I., and R.E. Johnson. Near-surface oxygen atmosphere at Europa. Adv. Space Res., 27, 1881-1888, 2001, DOI: 10.1016/S0273-1177(01)00299-X

Shematovich, V.I., R.E. Johnson, J.F. Cooper, and M.C. Wong. Surface-bounded atmosphere of Europa. Icarus, 173, 480-498, 2005, DOI: 10.1016/j.icarus.2004.08.013.

Showalter, M.R., J.A. Burns, J.N. Cuzzi, and J.B. Pollack. Jupiter's ring system - new results on structure and particle properties. Icarus, 69, 458-498, 1987, DOI: 10.1016/0019-1035(87)90018-2.

Sicard-Piet, A., S. Bourdarie, and N. Krupp. JOSE: a new Jovian specification environment model. IEEE Trans. Nucl. Sci., 58, 923-931, 2011, DOI: 10.1109/TNS.2010.2097276.

Simon, S., H. Kriegel, J. Saur, and A. Wennmacher. Energetic aspects of Enceladus' magnetospheric interaction. J. Geophys. Res. [Space Phys.], 118, 3430-3445, 2013a, DOI: $10.1002 /$ jgra.50380.

Simon, S., S.C. van Treeck, A. Wennmacher, J. Saur, F.M. Neubauer, C.L. Bertucci, and M.K. Dougherty. Structure of Titan's induced magnetosphere under varying background magnetic field conditions: survey of Cassini magnetometer data from flybys TA-T85. J. Geophys. Res., 118, 1679-1699, 2013b, DOI: 10.1002/jgra.50096.

Simon, S., J. Saur, S.C. Treeck, H. Kriegel, and M.K. Dougherty. Discontinuities in the magnetic field near Enceladus. Geophys. Res. Lett., 41, 3359-3366, 2014, DOI: 10.1002/2014GL060081.

Simonsen, L.C., and J.E. Nealy. Mars surface radiation exposure for solar maximum conditions and 1989 solar proton events. NASA Technical Paper 3300, 1993.

Singer, S.F. Trapped albedo theory of the radiation belt. Phys. Rev. Lett., 1, 181-183, 1958, DOI: 10.1103/PhysRevLett.1.181.

Sittler Jr., K.W. Ogilvie, E.C., and R. Selesnick. Survey of electrons in the Uranian magnetosphere - Voyager 2 observations. J. Geophys. Res. [Space Phys.], 92, 15263-15281, 1987, DOI: 10.1029/JA092iA13p15263.

Sittler Jr., M. Thomsen, E.C., R.E. Johnson, R.E. Hartle, M. Burger, et al. Cassini observations of Saturn's inner plasmasphere: saturn orbit insertion results. Planet. Space Sci., 541 (12), 1197-1210, 2006, DOI: 10.1016/j.pss.2006.05.038.

Sittler, E., R.P. Hartle, C. Bertucci, A. Coates, A. Cravens, I. Dandouras, and D. Shemansky. Energy deposition processes in Titans upper atmosphere and its induced magnetosphere. In: R.H. Brown, et al., Editors. Titan from Cassini-Huygens, Springer Science+Business Media B.V, Heidelberg, 393-453, 2009.

Sittler, E.C., J.F. Cooper, R.E. Hartle, W.R. Paterson, E.R. Christian, et al. Plasma ion composition measurements for Europa. Planet. Space Sci., 88, 26-41, 2013, DOI: 10.1016/j.pss.2013.01.013.

Slanger, T.G., P.C. Cosby, D.L. Huestis, and T.A. Bida. Discovery of the atomic oxygen green line in the Venus night airglow. Science, 291 (5503), 463-465, 2001.

Slanger, T.G., P.C. Cosby, D.L. Huestis, and R.R. Meier. Oxygen atom Rydberg emission in the equatorial ionosphere from radiative recombination. J. Geophys. Res. [Space Phys.], 109, A10309, 2004, DOI: 10.1029/2004JA010556.

Slavin, J.A., and R.E. Holzer. The effect of erosion on the solar wind stand-off distance at Mercury. J. Geophys. Res. [Space Phys.], 84 (A5), 2076-2082, 1979.

Slavin, J.A., and R.E. Holzer. Solar wind flow about the terrestrial planets 1. Modeling bow shock position and shape. J. Geophys. Res., 86 (A13), 11401-11418, 1981, DOI: $10.1029 /$ JA086iA13p11401.

Slavin, J.A., R.E. Holzer, J.R. Spreiter, and S.S. Stahara. Planetary mach cones: theory and observation. J. Geophys. Res., 89, 2708, 1984.

Slavin, J.A., B.J. Anderson, T.H. Zurbuchen, D.N. Baker, S.M. Krimigis, et al. MESSENGER observations of Mercury's magnetosphere during northward IMF. Geophys. Res. Lett., 36, L02101, 2009, DOI: 10.1029/2008GL036158. 
Slavin, J.A., B.J. Anderson, D.N. Baker, M. Benna, S.A. Boardsen, et al. MESSENGER observations of extreme loading and unloading of Mercury's magnetic tail. Science, 329 (5992), 665, 2010, DOI: 10.1126/science.1188067.

Slavin, J.A., P.C. Frisch, H.-R. Müller, J. Heerikhuisen, N.V. Pogorelov, W.T. Reach, and G. Zank. Trajectories and distribution of interstellar dust grains in the heliosphere. Astrophys. J., 760 (1), 46, 2012a.

Slavin, J.A., S.M. Imber, S.A. Boardsen, G.A. DiBraccio, and T. Sundberg. MESSENGER observations of a flux-transfer-event shower at Mercury. J. Geophys. Res., 117, A00M06, 2012b, DOI: $10.1029 / 2012 J A 017926$.

Slavin, J.A., G.A. DiBraccio, D.J. Gershman, S.M. Imber, G.K. Poh, et al. MESSENGER observations of Mercury's dayside magnetosphere under extreme solar wind conditions. J. Geophys. Res., 119, 8087-8116, 2014.

Smith, B.A., L.A. Soderblom, R. Beebe, J. Boyce, G. Briggs, et al. Galilean satellites and Jupiter - Voyager 2 imaging science results. Science, 206, 927-950, 1979.

Smith, D.E., M.T. Zuber, R.J. Phillips, S.C. Solomon, S.A. Hauck II, et al. Gravity field and internal structure of Mercury from MESSENGER. Science, 336, 214-271, 2012 DOI: $10.1126 /$ science. 1218809

Smith, E.J., L. Davis, D.E. Jones, P.J. Coleman, D.S. Colburn, P. Dyal, and C.P. Sonett. Saturn's magnetic field and magnetosphere. Science, 25, 407-410, 1980.

Smith, G.R., D.E. Shemansky, J.B. Holberg, A.L. Broadfoot, B.R. Sandel, and J.C. McConnell. Saturn's upper atmosphere from the Voyager 2 Euv solar and stellar occultations. J. Geophys. Res., 88 (A11), 8667-8678, 1983, DOI: 10.1029/JA088iA11p08667.

Smith, H.T., R.E. Johnson, M.E. Perry, D.G. Mitchell, R.L. McNutt, and D.T. Young. Enceladus plume variability and the neutral gas densities in Saturn's magnetosphere. J. Geophys. Res. [Space Phys.], 115, A10252, 2010, DOI: $10.1029 / 2009 J A 015184$.

Smyth, W.H., and M.L. Marconi. Europa's atmosphere, gas tori, and magnetospheric implications. Icarus, 181, 510-526, 2006, DOI: 10.1016/j.icarus.2005.10.019.

Snowden, D., and R.V. Yelle. The global precipitation of magnetospheric electrons into Titan's upper atmosphere. Icarus, 243, 1-15, 2014, DOI: 10.1016/j.icarus.2014.08.027.

Solomon, S.C., R.L. McNutt Jr., R.E. Gold, and D.L. Domingue. MESSENGER mission overview. Space Sci. Rev., 131, 3-39, 2007, DOI: 10.1007/s11214-007-9247-6.

Sonnerup, B.U.O. Magnetopause reconnection rate. J. Geophys. Res., 79 (10), 1546-1549, 1974,

DOI: 10.1029/JA079i010p01546.

Southwood, D.J., and M.G. Kivelson. A new perspective concerning the influence of the solar wind on the Jovian magnetosphere. J. Geophys. Res. [Space Phys.], 106, 6123-6130, 2001, DOI: $10.1029 / 2000 J A 000236$.

Southwood, D.J., and K.G. Kivelson. The source of Saturn's periodic radio emission. J. Geophys. Res. [Space Phys.], 114, A09201, 2009, DOI: 10.1029/2008JA013800

Stallard, T.S., S. Miller, S.W.H. Cowley, and E.J. Bunce. Jupiter's polar ionospheric flows: measured intensity and velocity variations poleward of the main auroral oval. Geophys. Res. Lett., 30, 1221, 2003, DOI: 10.1029/2002GL016031.

Steffl, A.J., P.A. Delamere, and F. Bagenal. Cassini UVIS observations of the Io plasma torus. IV. Observations of temporal and azimuthal variability. Icarus, 194, 153-165, 2008.

Stephan, K., R. Jaumann, R. Wagner, R.N. Clark, D.P. Cruikshank, et al. Dione's spectral and geological properties. Icarus, 206, 631-652, 2010, DOI: 10.1016/j.icarus.2009.07.036.

Stephan, K., R. Jaumann, R. Wagner, R.N. Clark, D.P. Cruikshank, et al. The Saturnian satellite Rhea as seen by Cassini VIMS. Planet. Space Sci., 61, 142-160, 2012, DOI: 10.1016/j.pss.2011.07.019.

Stiepen, A., J.-C. Gérard, M. Dumont, C. Cox, and J.-L. Bertaux. Venus nitric oxide nightglow mapping from SPICAV nadir observations. Icarus, 226 (1), 428-436, 2013, DOI: 10.1016/j.icarus.2013.05.031.

Stiepen, A., J.-C. Gérard, S. Bougher, F. Montmessin, B. Hubert, and J.-L. Bertaux. Mars thermospheric scale height: CO Cameron and $\mathrm{CO}_{2}{ }^{+}$dayglow observations from Mars Express. Icarus, 245 (1), 295-305, 2015, DOI: 10.1016/j.icarus.2014.09.051.

Storini, M., K. Kudela, E.G. Cordaro, and S. Massetti. Ground-level enhancements during solar cycle 23: results from SVIRCO, LOMNICKY STIT and LARC neutron monitors. Adv. Space Res., 35 (3), 416-420, 2005, DOI: 10.1016/j.asr.2004.12.020.

Suess, S.T., and B.E. Goldstein. Compression of the Hermean magnetosphere by the solar wind. J. Geophys. Res., 84, 3306-3312, 1979.

Sundberg, T., J.A. Slavin, S.A. Boardsen, B.J. Anderson, H. Korth, et al. MESSENGER observations of dipolarization events in Mercury's magnetotail. J. Geophys. Res., 117, A00M03, 2012, DOI: $10.1029 / 2012 J A 017756$.

Taylor, W.W.L., F.L. Scarf, C.T. Russell, and L.H. Brace. Evidence for lightning on Venus. Nature, 279, 614-616, 1979, DOI: $10.1038 / 279614 \mathrm{a} 0$.

Teolis, B.D., and J.H. Waite. Cassini measurements show seasonal $\mathrm{O}_{2}-\mathrm{CO}_{2}$ exospheres and possible seasonal $\mathrm{CO}_{2}$ frosts at Rhea and Dione. 43rd Lunar Planet. Sci. Conf., Lunar and Planetary Institute, Houston, Texas, 2923, 2012.

Teolis, B.D., and J.H. Waite. Dione and Rhea seasonal exospheres revealed by Cassini CAPS and INMS. Icarus, 272, 277-289, 2016, DOI: $10.1016 /$ j.icarus.2016.02.031.

Teolis, B.D., G.H. Jones, P.F. Miles, R.L. Tokar, B.A. Magee, et al. Cassini finds an oxygen-carbon dioxide atmosphere at Saturn's Icy Moon Rhea. Science, 330, 1813, 2010, DOI: $10.1126 /$ science. 1198366.

Tokar, R.L., R.E. Johnson, T.W. Hill, D.H. Pontius, W.S. Kurth, et al. The interaction of the atmosphere of Enceladus with Saturn's plasma. Science, 311, 1409-1412, 2006, DOI: $10.1126 /$ science.1121061.

Tokar, R.L., R.E. Johnson, M.F. Thomsen, E.C. Sittler, A.J. Coates, R.J. Wilson, F.J. Crary, D.T. Young, and G.H. Jones. Detection of exospheric $\mathrm{O}_{2}{ }^{+}$at Saturn's moon Dione. Geophys. Res. Lett., 39, L03105, 2012, DOI: 10.1029/2011GL050452.

Trafton, L.M., S. Miller, T.R. Geballe, J. Tennyson, and G.E. Ballester. $\mathrm{H}_{2}$ Quadrupole and $\mathrm{H}_{3}{ }^{+}$emission from Uranus: the uranian thermosphere, ionosphere, and auror. Astrophys. J., 524, 1059-1083, 1999, DOI: 10.1086/307838.

Turc, L., L. Leclercq, F. Leblanc, R. Modolo, and J.Y. Chaufray. Modelling Ganymede's neutral environment: a 3D test-particle simulation. Icarus, 229, 157-169, 2014.

Turrini, D., R. Politi, R. Peron, D. Grassi, C. Plainaki, et al. The comparative exploration of the ice giant planets with twin spacecraft: unveiling the history of our Solar System. Planet. Space Sci., 104, 93-107, 2014, DOI: 10.1016/j.pss.2014.09.005.

Van Allen, J.A. Absorption of energetic protons by Saturn's Ring G. J. Geophys. Res. [Space Phys.], 88, 6911-6918, 1983, DOI: 10.1029/JA088iA09p06911.

Van Allen, J.A. Energetic particles in the inner magnetosphere of Saturn. In: T. Gehrels, and M.S. Matthews, Editors. Saturn, The University of Arizona Press, Tucson, Arizona, 281-317, 1984.

Vasyliunas, V.M. A survey of low-energy electrons in the evening sector of the magnetosphere with OGO 1 and OGO 3. J. Geophys. Res. [Space Phys.], 73, 2839-2884, 1968, DOI: $10.1029 / \mathrm{JA} 073 \mathrm{i} 009 \mathrm{p} 02839$.

Vasyliunas, V.M. Plasma distribution and flow. In: A.J. Dessler, Editor. Physics of the Jovian magnetosphere, Cambridge University Press, A83-26611 10-91, Cambridge and New York, 395-453, 1983.

Vasyliunas, V.M. The convection-dominated magnetosphere of Uranus. Geophys. Res. Lett., 13, 621-623, 1986 , DOI: $10.1029 /$ GL013i007p00621.

Vervack, R.J., W.E. McClintock, R.M. Killen, A.L. Sprague, B.J. Anderson, et al. Mercury's complex exosphere: results from MESSENGER's third flyby. Science, 329 (5992), 672, 2010, 
Vidal-Madjar, A., A. Lecavelier des Etangs, J.-M. Désert, G.E. Ballester, R. Ferlet, et al. An extended upper atmosphere around the extrasolar planet HD209458b. Nature, 422, 143-146, 2003.

Vigren, E., M. Galand, R.V. Yelle, J. Cui, J.-E. Wahlund, et al. On the thermal electron balance in Titan's sunlit upper atmosphere. Icarus, 223, 234-251, 2013, DOI: $10.1016 /$ j.icarus.2012.12.010.

Vilas, F., C.R. Chapman, and M. Mathews. Mercury. Univ. Arizon Press, Tucson, Arizona, USA, 1988.

Vogt, M.F., M.G. Kivelson, K.K. Khurana, S.P. Joy, and R.J. Walker, Reconnection and flows in the Jovian magnetotail as inferred from magnetometer observations. J. Geophys. Res., 115 (A6), A06219, 2010, DOI: 10.1029/2009JA015098

Voigt, G.-H., T.W. Hill, and A.J. Dessler. The magnetosphere of Uranus - plasma sources, convection, and field configuration. Astrophys. J., 266, 390-401, 1983, DOI: 10.1086/160787.

Volwerk, M., X. Jia, C. Paranicas, W.S. Kurth, M.G. Kivelson, and K.K. Khurana. ULF waves in Ganymede's upstream magnetosphere. Ann. Geophys., 31, 45-59, 2013.

Von Zahn, U., S. Kumar, H. Niemann, and R. Prinn. Composition of the Venus atmosphere. In: D.M. Hunten, L. Colin, T.M. Donahue, and V. I. Moroz, Editors. Venus, University of Arizona Press, Tucson, USA, 779-840, 1983.

Vorburger, A., P. Wurz, H. Lammer, S. Barabash, and O. Mousis. Monte-Carlo simulation of Callisto's exosphere. Icarus, 262, 14-29, 2015, DOI: 10.1016/j.icarus.2015.07.035.

Waite Jr., J.H., and T.E. Cravens. Current review of the Jupiter, Saturn, and Uranus ionospheres. Adv. Space Res., 7 (12), 119-134, 1987, DOI: 10.1016/0273-1177(87)90210-9.

Waite Jr., T.E. Cravens, J.H., J. Kozyra, A.F. Nagy, S.K. Atreya, and R.H. Chen. Electron precipitation and related aeronomy of the Jovian thermosphere and ionosphere. J. Geophys. Res., 88 (8), 6143-6163, 1983, DOI: 10.1029/JA088iA08p06143.

Waite, J.H., G.R. Gladstone, W.S. Lewis, R. Goldstein, D.J. McComas, et al. An auroral flare at Jupiter. Nature, 410, 787-789, 2001.

Waite, J.H., W.S. Lewis, W.T. Kasprzak, V.G. Anicich, B.P. Block, et al., The Cassini Ion and Neutral Mass Spectrometer (INMS) Investigation, Space Sci. Rev., 114, 113-231, 2004, DOI: $10.1007 / \mathrm{s} 11214-004-1408-2$.

Waite, J.H., H. Niemann, R.V. Yelle, W.T. Kasprzak, T.E. Cravens, et al. Ion Neutral Mass Spectrometer results from the first flyby of Titan. Science, 308, 982-986, 2005, DOI: $10.1126 /$ science.1110652.

Waite Jr., M.R. Combi, J.H., W.H. Ip, T.E. Cravens, R.L. McNutt Jr., et al. Cassini ion and neutral mass spectrometer: enceladus plume composition and structure. Science, 311, 1419-1422, 2006.

Waite, J.H., D.T. Young, T.E. Cravens, A.J. Coates, F.J. Crary, B. Magee, and J. Westlake. The process of tholin formation in Titan's upper atmosphere. Science, 316, 870-875, 2007.

West, R.A., J.M. Ajello, M.H. Stevens, D.F. Strobel, G.R. Gladstone, J.S. Evans, and E.T. Bradley. Titan airglow during eclipse. Geophys. Res. Lett., 39 (1), 18204, 2012.

Whitten, R.C., I.G. Poppoff, and J.S. Sims. The ionosphere of mars below $80 \mathrm{~km}$ altitude - I quiescent conditions. Planet. Space Sci., 19, 243-250, 1971, DOI: 10.1016/0032-0633(71)90203-0.

Winslow, R.M., B.J. Anderson, C.L. Johnson, J.A. Slavin, H. Korth, M.E. Purucker, D.N. Baker, and S.C. Solomon. Mercury's magnetopause and bow shock from MESSENGER magnetometer observations. J. Geophys. Res. [Space Phys.], 118 (5), 2213-2227, 2013, DOI: 10.1002/jgra.50237.
Withers, P. Attenuation of radio signals by the ionosphere of Mars: theoretical development and application to MARSIS observations. Radio Sci., 46, RS2004, 2011, DOI: $10.1029 / 2010$ RS004450.

Woch, J., N. Krupp, A. Lagg, B. Wilken, S. Livi, and D.J. Williams. Quasi-periodic modulations of the Jovian magnetotail. Geophys. Res. Lett, 25, 1253-1256, 1998.

Woch, J., N. Krupp, K.K. Khurana, M.G. Kivelson, and A. Roux. Plasma sheet dynamics in the Jovian magnetotail: signatures for substorm-like processes? Geophys. Res. Lett., 26, 2137-2140, 1999.

Woch, J., N. Krupp, and A. Lagg. Particle bursts in the Jovian magnetosphere: evidence for a near-Jupiter neutral line. Geophys. Res. Lett., 29 (7), 1138, 2002, DOI: 10.1029/2001GL014080.

Wong, M.C., and R.E. Johnson. A three-dimensional azimuthally symmetric model atmosphere for Io 1 . Photochemistry and the accumulation of a nightside atmosphere. J. Geophys. Res. [Space Phys.], 101, 23243-23254, 1996, DOI: 10.1029/96JE02510.

Woodfield, E.E., R.B. Horne, S.A. Glauert, J.D. Menietti, and Y.Y. Shprits. The origin of Jupiter's outer radiation belt. J. Geophys. Res. [Space Phys.], 119, 3490-3502, 2014, DOI: $10.1002 / 2014 J A 019891$.

Wu, X.-Y., J.L. Horwitz, and J.-N. Tu. Dynamic fluid kinetic (DyFK) simulation of auroral ion transport: synergistic effects of parallel potentials, transverse ion heating, and soft electron precipitation, J. Geophys. Res., 107 (A10), 1283-2002, DOI: 10.1029/2000JA000190.

Wurz, P., and H. Lammer. Monte-Carlo simulation of Mercury's exosphere. Icarus, 164, 1-13, 2003.

Wurz, P., J.A. Whitby, U. Rohner, J.A. Martín-Fernández, H. Lammer, and C. Kolb. Self-consistent modelling of Mercury's exosphere by sputtering, micro-meteorite impact and photonstimulated desorption. Planet. Space Sci., 58 (12), 1599-1616, 2010.

Yair, Y. New results on planetary lightning. Adv. Space Res., 50, 293-310, 2012, DOI: 10.1016/j.asr.2012.04.013.

Yair, Y., G. Fischer, F. Simões, N. Renno, and P. Zarka. Updated review of planetary atmospheric electricity. Space Sci. Rev., 137, 29-49, 2008, DOI: 10.1007/s11214-008-9349-9.

Yamauchi, M., and J.-E. Wahlund. Role of the ionosphere for the atmospheric evolution of planets. Astrobiology, 7 (5), 783-800, 2007, DOI: 10.1089 /ast.2007.0140.

Young, L.A., R.V. Yelle, R. Young, A. Seiff, and D.B. Kirk. Gravity waves in Jupiter's thermosphere. Science, 276 (5309), 108-111, 1997.

Zarka, P. Auroral radio emissions at the outer planets: observations and theories, J. Geophys. Res. [Planets], 103 (E9), 20159-20194, 1998.

Zarka, P., B.M. Pedersen, A. Lecacheux, M.L. Kaiser, M.D. Desch, W.M. Farrell, and W.S. Kurth. Radio emissions from Neptune. In: D.P. Cruikshank, M.S. Matthews, and A.M. Schumann, Editors. Neptune and Triton, Astronomisches Rechen-Institut Publisher, Germany, 341-387, 1995.

Zurbuchen, T.H., J.M. Raines, G. Gloeckler, S.M. Krimigis, J.A. Slavin, et al. MESSENGER observations of the composition of Mercury's ionized exosphere and plasma environment. Science, 321 (5885), 90, 2008.

Zurbuchen, T.H., J.M. Raines, J.A. Slavin, D.J. Gershman, J.A. Gilbert, et al. MESSENGER observations of the spatial distribution of planetary ions near Mercury. Science, 333 (6051), 1862,2011

Zwan, B.J., and R.A. Wolf. Depletion of solar-wind plasma near a planetary boundary. J. Geophys. Res., 81, 1636, 1976.

Cite this article as: Plainaki C, Lilensten J, Radioti A, Andriopoulou M, Milillo A, et al. Planetary space weather: scientific aspects and future perspectives. J. Space Weather Space Clim., 6, A31, 2016, DOI: 10.1051/swsc/2016024. 